\title{
Measurement of the Parity-Violating Asymmetry in Deep Inelastic Scattering at JLab $6 \mathrm{GeV}$
}

\author{
Diancheng Wang \\ Yangzhong, Jiangsu, China \\ B.S. in Physics, Nanjing University, 2007 \\ A Dissertation presented to the Graduate Faculty \\ of the University of Virginia in Candidacy for the Degree of \\ Doctor of Philosophy \\ Department of Physics \\ University of Virginia
}

December 2013 


\begin{abstract}
The parity-violating asymmetry in deep inelastic scattering (PVDIS) offers us a useful tool to study the weak neutral couplings and the hadronic structure of the nucleon, and provides high precision tests on the Standard Model. During the $6 \mathrm{GeV}$ PVDIS experiment at the Thomas Jefferson National Accelerator Facility, the parity-violating asymmetries $A_{P V}$ of a polarized electron beam scattering off an unpolarized deuteron target in the deep inelastic scattering region were precisely measured at two $Q^{2}$ values of 1.1 and $1.9(\mathrm{GeV} / \mathrm{c})^{2}$. The asymmetry at $Q^{2}=1.9(\mathrm{GeV} / \mathrm{c})^{2}$ can be used to extract the weak coupling combination $2 C_{2 u}-C_{2 d}$, assuming the higher twist effect is small. The extracted result from this measurement is in good agreement with the Standard Model prediction, and improves the precision by a factor of five over previous data. In addition, combining the asymmetries at both $Q^{2}$ values provides us extra knowledge on the higher twist effects.

The parity violation asymmetries in the resonance region were also measured during this experiment. These results are the first $A_{P V}$ data in the resonance region beyond the $\Delta(1232)$. They provide evidence that the quark-hadron duality works for $A_{P V}$ at the (10-15)\% level, and set constraints on nucleon resonance models that are commonly used for background calculations to other parity-violating electron scattering measurements.
\end{abstract}




\section{Acknowledgments}

I simply can't start my acknowledgments with anyone else other than my advisor, Xiaochao Zheng, who has been such a terrific physicist, mentor and friend in the past years. Without her guidance and help, both in research and in everyday life, I wouldn't have reached this point. Pursuing a $\mathrm{PhD}$ is a challenging journey, both mentally and intellectually, but her always being there to help made this trip so much easier. For that, I will forever be thankful.

I would also like to thank the wonderful staff of Jefferson Lab. Especially, I would like to thank Robert Micheals, one of the spokespeople of PVDIS and the expert of experts in data acquisition, from whom I learned so much in both physics ideas and programming skills. I also want to thank Alexandre Camsonne, whose company made the long nights working at the counting house much more enjoyable, and whose bread and cheese kept me from getting any skinnier during the stressful period of experiment running.

Thanks to all the people of the parity collaboration, especially the leading experts: Kent Paschke, Paul Souder, Krishna Kumar, David Armstrong and Paul Reimer, whose experience and physics insights played significant roles throughout the experiment, from DAQ construction and data collection to data analysis and results publishing. As part of the parity family, PVDIS benefited a great deal from the close collaboration with its siblings HAPPEX-III and PREX, which guaranteed its success.

Of course, there's no way to leave out the main manpower that made this experiment happen: Ramesh Subedi, Kai Pan, Xiaoyan Deng and Huaibo Ding, as well as the postdocs and students from HAPPEX-III and PREX: Mark Dalton, Dustin McNulty, Rupesh Silwal, Megan Friend, Luis Mercado, Abdurahim Rakhman, Zafar Ahmed, Chun-Min Jen and Kiadtisak Saenboonruang. It is the hard work of this 
intelligent and diligent group of people that made everything possible.

Lastly, I would like to thank my dear parents, Guocai Wang and Chunying Yang, and my sister, Fang Wang, for their unconditional love and support. Their encouragement is the major source of power that led me to overcome all the life challenges and become who I am today. 


\section{Dedication}

To my ever so loving parents, Guocai Wang and Chunying Yang. 


\section{Contents}

List of Figures $\quad$ viii

List of Tables $\quad$ XV

1 Introduction $\quad 1$

1.1 Deep Inelastic Electron Scattering . . . . . . . . . . . . . . . . . . 3

1.1.1 Electron Scattering . . . . . . . . . . . . . . 3

1.1.2 Unpolarized DIS Cross Section and Structure Functions . . . . 7

1.1.3 The Quark Parton Model . . . . . . . . . . . . . . 8

1.2 PVDIS Formalism . . . . . . . . . . . . . . . . . . . . . 9

1.3 Current knowledge on $C_{1 q}$ and $C_{2 q}$ Couplings . . . . . . . . . 15

$1.4 A_{\mathrm{PV}}$ in the Resonance Region . . . . . . . . . . . . . . . . 17

2 Experimental Setup 19

2.1 Accelerator ....................... . . . . 20

2.2 Experimental Hall A . . . . . . . . . . . . . . . . . . 21

2.3 Beamline . . . . . . . . . . . . . . . . . . . . 22

2.3.1 Beam Helicity . . . . . . . . . . . . . . . . . . 22

2.3.2 Beam Energy Measurement .............. . . . 24

2.3.3 Beam Current . . . . . . . . . . . . . . 26

2.3.3.1 Beam Current Monitors . . . . . . . . . . 26

2.3.3.2 Charge Asymmetry Feedback . . . . . . . . . . 28

2.3.4 Beam Position . . . . . . . . . . . . . . . . 29

2.3.4.1 Beam Position Monitors . . . . . . . . . . 29

2.3.4.2 Beam Modulation ............. . . 30

2.3.4.3 Raster . . . . . . . . . . . . . . . . . . . . . . . . . . . . . . 31

2.3.5 Beam Polarization ................. . . . 31

2.3.5.1 Mott Polarimeter . . . . . . . . . . . . . . . 32

2.3.5.2 Møller Polarimeter . . . . . . . . . . . . . . . . . . . 33

2.3.5.3 Compton .................. 36

2.4 Target . . . . . . . . . . . . . . . . . . . . 38

2.4.1 Cryogenic Target System . . . . . . . . . . . . . . . . . 38

2.4.2 Target Boiling . . . . . . . . . . . . . . . 40 
2.5 High Resolution Spectrometers _. . . . . . . . . . . . . . 43

2.5 .1 Optics Magnets . . . . . . . . . . . . . . . . . . 44

2.5.2 Detector Package . . . . . . . . . . . . . . . . . . 47

2.5.2.1 Vertical Drift Chambers . . . . . . . . . . 47

2.5.2.2 Scintillators . . . . . . . . . . . . . . . 49

2.5.2.3 Gas Cerenkov . . . . . . . . . . . . . . . 50

2.5.2.4 Lead Glass Counters . . . . . . . . . . . . . . 51

2.6 Data Acquisition System . . . . . . . . . . . . . . . . . . . . . . . . 52

2.6 .1 HRS DAQ . . . . . . . . . . . . . . . . . . . . 53

2.6 .2 Parity DAQ . . . . . . . . . . . . . . . . . 54

3 Data Analysis $\quad 59$

3.1 Overview . . . . . . . . . . . . . . . . . . . . . . . . . . . . . 59

3.1 .1 Overview of Data Taking . . . . . . . . . . . . . . 59

3.1 .2 Overview of Data Analysis . . . . . . . . . . . . . 61

3.2 Raw Asymmetry . . . . . . . . . . . . . . . . . . . 63

3.3 Forming Raw Asymmetries . . . . . . . . . . . . . . . . . . . . 63

3.3 .1 Data Selection . . . . . . . . . . . . . . . . . . . . 64

3.3 .2 BCM Calibration . . . . . . . . . . . . . . . . . 68

3.4 False Asymmetry and Corrections . . . . . . . . . . . . . . . . . 69

3.4 .1 Charge Asymmetry . . . . . . . . . . . . . . . . . . . 69

3.4.2 Helicity Correlated Beam Fluctuations . . . . . . . . . . . 69

3.4.2.1 Beam Modulation (Dithering) . . . . . . . . . . 70

3.4.2.2 Linear Regression . . . . . . . . . . . . . . . 72

3.5 Statistical Quality of Data . . . . . . . . . . . . . . 74

3.6 Beam Polarization . . . . . . . . . . . . . . . . . . . . 77

3.6.1 Compton Measurements (Photon Detector) . . . . . . . . . 77

3.6.1.1 Extraction of $A_{\text {exp }} \ldots \ldots \ldots \ldots \ldots$

3.6.1.2 Calculation of $A_{t h} \ldots \ldots \ldots \ldots \ldots$

3.6.1.3 Laser Polarization . . . . . . . . . . . . . . 82

3.6.1.4 Compton Results . . . . . . . . . . . . . . . . . 82

3.6.2 Møller Measurements . . . . . . . . . . . . . . . . . . . . . 83

3.6.3 Combining Compton and Møller . . . . . . . . . . . . . 84

3.7 Deadtime Correction . . . . . . . . . . . . . . . . . . 85

3.7.1 Decomposition of the Deadtime . . . . . . . . . . . 86

3.7.2 Methods to study the deadtime . . . . . . . . . . 87

3.7.2.1 Group Deadtime: The Tagger Method . . . . . . . 87

3.7.2.2 VETO Deadtime: Flash ADC Method . . . . . . . 90

3.7.2.3 OR Deadtime: Analytical Analysis . . . . . . . . . 91

3.7.2.4 Software Simulation . . . . . . . . . . . . . . . 93

3.7 .3 DATA-Simulation Comparison . . . . . . . . . . . . . . . . 93

3.7.4 Deadtime Results . . . . . . . . . . . . . . . . . . . . . . . . . 98

3.8 Particle Identification . . . . . . . . . . . . . . . . . . . . . . . . 98 
3.9 Calibration of the HRS Optics . . . . . . . . . . . . . . 101

3.9.1 Optics Calibration Procedure and the Resulting Systematic Un-

certainties . . . . . . . . . . . . . . 101

3.9.1.1 Optics Calibration Results . . . . . . . . . 105

3.9.1.2 $Q^{2}$ Uncertainties . . . . . . . . . . . . . 106

3.10 Hall A Monte Carlo . . . . . . . . . . . . . . . . . . . . . . . . . 109

3.11 Electromagnetic Radiative Correction . . . . . . . . . . . . . . . . . 111

3.11.1 Beam Depolarization Effect in Bremsstralung . . . . . . . . . 113

3.11.2 Corrections for Vertex versus Detected Kinematics . . . . . . 115

3.11.3 Box Diagram Corrections . . . . . . . . . . . . . . . . . 120

3.12 Background Analysis . . . . . . . . . . . . . . . . . . . . . . . . 122

3.12.1 Charged Pion Background . . . . . . . . . . . . . . . 122

3.12.2 Pair Production Background . . . . . . . . . . . . . . . 126

3.12.3 Target EndCap Corrections . . . . . . . . . . . . . . . . . . . 128

3.12.4 Beam Transverse Asymmetry Background . . . . . . . . . . . 131

3.12.5 Target Purity, Density Fluctuation and Other False Asymmetries 134

3.12.6 Rescattering and Poletip Scattering Background . . . . . . . . 134

3.13 Resonance Analysis using Group Triggers . . . . . . . . . . . . . . . . 139

4 Results and Conclusions $\quad 142$

4.1 Resonance Results . . . . . . . . . . . . . . . . . . . . . 143

4.2 DIS Results . . . . . . . . . . . . . . . . . . . . . . 148

4.2.1 DIS Asymmetry Results . . . . . . . . . . . . . . . . 148

4.2.2 Standard Model Calculations And the Extraction of Quark Ef-

fective Couplings $2 C_{2 u}-C_{2 d} \ldots \ldots \ldots$. . . . . . . . 148

4.2.2.1 Electroweak Radiative Corrections . . . . . . . . . 148

4.2.2.2 Evaluation of Structure Functions and Extraction of

$C_{2 q} \ldots \ldots \ldots \ldots \ldots . \ldots \ldots$

4.2.2.3 The Higher Twist Effect . . . . . . . . . . . . . . . 154

4.3 Summary . . . . . . . . . . . . . . . . . 156

$\begin{array}{lr}\text { Bibliography } & 157\end{array}$ 


\section{List of Figures}

1.1 Feynman diagram of electron scattering through one-photon exchange. 4

1.2 Feynman diagram of electron scattering including both electromagnetic and weak interactions. . . . . . . . . . . . . . . . 99 9

1.3 Existing constrains on the weak coupling constants $C_{1 q}$ and $C_{2 q}$. The $C_{1 q}$ plot (a) is from [24]. . . . . . . . . . . . . . 16

2.1 The Jefferson Lab accelerator. . . . . . . . . . . . . . . . . . . 20

2.2 The layout of Hall A during PVDIS. . . . . . . . . . . . . . . . . . . 22

2.3 (a): Structure of the Helicity signal and the delayed Helicity signal; (b): Timing of the MPS signal with respect to the Helicity signal. The MPS signal is used to mute the transition period during change of Helicity states. . . . . . . . . . . . . . . . . .

2.4 Beam energy versus run number. The left and right plots represent two different beam energy settings during PVDIS. . . . . . . . . . . . 24

2.5 Schematic of beam current monitors. . . . . . . . . . . . . . . 26

2.6 Beam charge asymmetry feedback system. . . . . . . . . . . . . 28

2.7 Layout of the beam position monitors. . . . . . . . . . . . . . . . . . 29

2.8 One example cycle of the beam modulation ("dithering") procedure. . 30

2.9 Beam raster during PVDIS. . . . . . . . . . . . . . . . . . 32

2.10 Layout of the Møller polarimeter . . . . . . . . . . . . . . . . . 34

2.11 Layout of the Compton polarimeter . . . . . . . . . . . . . . 36

2.12 Schematic diagram of the target ladder arrangement used during the experiment. The electron beam is along the horizontal direction (the $z$-axis) and is incident from the left on the target. The carbon multifoils were located at $z=(-15,-7,0,7,15) \mathrm{cm}$ and the Al dummy foils were located at $z=(-10,10) \mathrm{cm}$. All other solid targets were located at $z=0 \mathrm{~cm}$ and were about 1 inch apart in the vertical direction. . 
2.13 Measured standard deviation of the pair-wise asymmetries at kinematics DIS\# 2, for various raster sizes and two beam currents 100 and $115 \mu \mathrm{A}$. The curves show results of fit $\delta A=p_{0} x^{p_{1}}+p_{2}$ where $x$ is the raster size (side size of square) in $\mathrm{mm}$. The fit results for $100 \mu \mathrm{A}$ are $p_{0}=(1.77 \pm 1.94) \times 10^{4}, p_{1}=-2.48 \pm 1.85, p_{2}=27973.0 \pm 681.7$; and for $115 \mu \mathrm{A}$ are $p_{0}=(9.40 \pm 3.78) \times 10^{3}, p_{1}=-1.37 \pm 1.09$, $p_{2}=25941.0 \pm 1433.4$. At a raster size of $4 \times 4 \mathrm{~mm}^{2}(x=4)$, the boiling noise is at the level of $569 \mathrm{ppm}(1407 \mathrm{ppm})$ for $100(115) \mu \mathrm{A}$, and is negligible compared to the value from purely statistical fluctuations (the $p_{0}$ ).

2.14 Measured standard deviation of the pair-wise asymmetries at various beam currents for DIS\# 1 (top) and \# 2 (bottom), with a $4 \times 4 \mathrm{~mm}^{2}$ square raster. The curves show results of fit $\delta A \propto I^{p_{0}}$ and its error. The fit results are $p_{0}=0.4900 \pm 0.0076$ and $p_{0}=0.4897 \pm 0.0072$ for DIS\# 1 and \# 2 respectively. These results are in good agreement

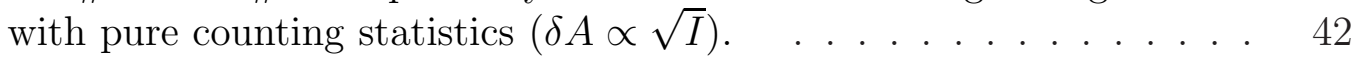

2.15 Layout of the HRS spectrometer. . . . . . . . . . . . . . . . 44

2.16 Schematic diagrams of the VDC configuration. . . . . . . . . . . . . 48

2.17 Configuration of the pion rejectors on the left HRS (top) and the preshower/shower detectors on the right HRS (bottom). . . . . . . . .

2.18 Grouping scheme (side-view) for the double-layer lead-glass detectors for the Left and the Right HRS. Scattered particles enter the detector from the left. The colored vertical bars represent the range of each group. . . . . . . . . . . . . . . . . .

2.19 Schematic diagram of the trigger logic of the Parity DAQ. See the text for detailed explanation.

3.1 Illustration of the BCM calibration. Left: raw ADC readings for the Unser monitor plotted versus event number during the BCM calibration procedure; Right: raw ADC readings for bcm1 plotted versus that for the Unser monitor during BCM calibration. The red line shows the linear fit $\mathrm{bcm} 1=\mathrm{p} 0+\mathrm{p} 1 \times$ Unser. The fit is only performed with data at high current. The fitting result p0 represents the pedestal value of the bcm1 ADC reading. . . . . . . . . . . . . . . .

3.2 Beam position differences $\Delta M_{i}$ measured by the five BPMs throughout the PVDIS experiment, plotted versus the slug number. . . . . . . . .

3.3 Pull plots of the global electron narrow trigger for different kinematics. The shaded areas represent the histograms for the pull $p_{i}$, and the red curves are Gaussian fits to the histograms. . . . . . . . . . . . . .

3.4 Schematic drawing of the background shielding for the Compton photon detector during PVDIS. The drawing is not to scale. . . . . . . . 79

3.5 Compton spectrum. . . . . . . . . . . . . . . . . . . . 81 
3.6 Beam polarization history from the Compton polarimeter (black solid circles), plotted with the Møller results (red open squares) available at the same time period. The error bars for Compton are statistical only, while for Møller they include systematic uncertainties. A constant fit to Compton measurements gave an average of $89.45 \%$ while the average of Møller results was $88.74 \%$. . . . . . . . . . . . . . . .

3.7 Polarization history from the Møller polarimeter measurements taken with a beam energy of $6.067 \mathrm{GeV}$. The error bars include systematic error. An additional measurement was done with a beam energy of $4.867 \mathrm{GeV}$ at the end of the run period, which gave similar polarization but is not shown here. . . . . . . . . . . . . . . . .

3.8 Top: schematic diagram for the tagger setup and signal timing sequence. Bottom: fbTDC spectrum for the relative timing between tagger-trigger coincidence and the input tagger, in 0.5-ns bins. The fbTDC module works in the multi-hit mode. Two different scenarios are shown: 1) Main peak $I_{0}$ : when there is no PMT signal preceding the tagger, the tagger triggers the DAQ and forms a tagger-trigger coincidence. 2) Pileup events $I_{1}$ and $I_{2}$ : when there is a PMT signal preceding the tagger by a time interval shorter than the delayed tagger width, the PMT signal triggers the DAQ and forms a tagger-trigger coincidence signal with the delayed tagger. . . . . . . . . . . .

3.9 The VETO circuits of the Parity DAQ for Left (top) and Right (bottom) spectrometers. . . . . . . . . . . . . . .

3.10 FADC snapshots of one example event that suffered VETO deadtime. The event triggered Cerenkov, Scintillator and the Preshower and Shower, meaning it was a good electron. However, the DAQ fails to generate the VETO signal due to the VETO deadtime, which results in the loss of the electron trigger. The early timing of the Scintillator signal indicates that it was from a non-electron-triggered signal that preceded the electron. . . . . . . . . . . . . . . 
3.11 Deadtime loss in percent vs. event rate from the tagger method for group 4 on the Left HRS. Top: actual deadtime loss from tagger measurements; Bottom: simulated deadtime loss of the tagger. The tagger fractional count loss $1-R_{o} / R_{i}$ (red) and the pileup correction $p$ (black) are combined to form the total group deadtime $D$ (blue). These data were taken (or simulated) at a $Q^{2}$ of $1.1(\mathrm{GeV} / c)^{2}$. To minimize the statistical uncertainty while keeping the computing time reasonable, the simulation used higher event rates than the tagger measurement. The total group deadtime can be determined from the linear fit slope coefficients: tagger data narrow $p_{1}=(61.5 \pm 0.2) \times 10^{-9} \mathrm{~s}$, wide $p_{1}=(99.9 \pm 0.3) \times 10^{-9} \mathrm{~s}$, simulation narrow $p_{1}=(62.5 \pm 1.4) \times 10^{-9} \mathrm{~s}$, wide $p_{1}=(102 \pm 1.3) \times 10^{-9} \mathrm{~s}$. Group 4 is from the central blocks of the lead-glass detector and has the highest rate among all groups. . .

3.12 Deadtime loss in percent vs. event rate from the tagger method for group 4 on the Right HRS. Top: tagger data; Bottom: simulation. These data were taken (or simulated) at a $Q^{2}$ of $1.9(\mathrm{GeV} / c)^{2}$. The total group deadtime can be determined from the linear fit slope coefficients: tagger data narrow $p_{1}=(71.1 \pm 0.9) \times 10^{-9} \mathrm{~s}$, wide $p_{1}=$ $(107 \pm 1.2) \times 10^{-9} \mathrm{~s}$, simulation narrow $p_{1}=(73.9 \pm 1.5) \times 10^{-9} \mathrm{~s}$, wide $p_{1}=(115 \pm 1.5) \times 10^{-9} \mathrm{~s}$. Group 4 is from the central blocks of the lead-glass detector and has the highest rate among all groups. See Fig. 3.11 caption for details. . . . . . . . . . . . . . . .

3.13 Timing difference between Scintillator and Cerenkov signals in 5-ns channels extracted from FADC data. The fractional loss of electron events due to VETO deadtime can be estimated using the ratio of $N_{1} / N_{0}$, where $N_{1}$ is the count difference between the two spectra in the dead zone, and $N_{0}$ is the counts under the main peak near 0 ns. .

3.14 VETO deadtime for both HRS from FADC analysis. Shown here is the comparison among simulation, data and theoretical calculation using Equation 3.7.7. . . . . . . . . . . . . .

3.15 Total deadtime results for all kinematics obtained from simulation, for narrow (red open triangles, fit by dashed red lines) and wide (blue open squares, fit by solid blue lines) paths. . . . . . . . . . . .

3.16 Top view of the target coordinate system (TCS) $\left(x_{t g}, y_{t g}, z_{t g}\right)$ and the sieve plane coordinate system $\left(x_{\text {sieve }}, y_{\text {sieve }}\right)$. 
3.17 Top: vertex reconstruction for Left DIS\#1 (left), where the observed foil position miss the actual values by $\Delta z_{\text {foil data }} \leqslant 0.4 \mathrm{~mm}$. Bottom: reconstruction of the sieve hole positions (scattered points) compared to the expected positions (grids), for Left RES II. No obvious discrepancy is seen. The axes are oriented such that the sieve hole pattern is as if viewed when facing the spectrometer entrance. Two of the sieve holes are larger than others to allow identifying the center and the orientation of the sieve plate. . . . . . . . . . . . . . . . .

3.18 Fine-tuning of the HRS acceptance in HAMC. Event distributions from data are plotted at the collimator (entrance of the HRS $Q_{1}$ ), $Q_{1}$ exit, and entrances and exits of the dipole, $Q_{2}$ and $Q_{3}$. Black dashed lines show the position, shape, and size of apertures determined by matching to the data distributions, which were then used as aperture or acceptance cuts in HAMC. The axes are oriented such that the distributions are as viewed along the particle trajectory, with $y$ the horizontal and

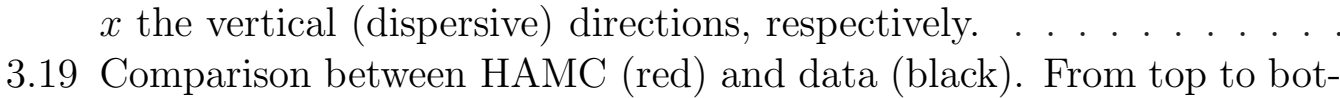
tom: target variables $-\theta_{\mathrm{tg}}, \phi_{\mathrm{tg}}, y_{\mathrm{tg}}$ and $(\delta p / p)_{\mathrm{tg}}-$ for Left HRS DIS\#1; $Q^{2}$ and $x$ for Left HRS DIS\#1; target variables for Right HRS DIS\#2; $Q^{2}$ and $x$ for Right HRS DIS\#2. . . . . . . . . . .

3.20 Kinematics used in HAMC to correct energy losses $\delta E$ and $\delta E^{\prime}$, respectively for the incoming and outgoing electrons. The kinematics reconstructed from the data corresponds to $E_{\text {beam }}$ and $E_{\text {det }}^{\prime}$, while the vertex kinematics corresponds to $E_{v}$ and $E_{v}^{\prime}$. . . . . . . . . . . .

3.21 Simulated vertex kinematics of the two DIS kinematics \#1 (left) and \#2 (right) . . . . . . . . . . . . . . . . . . . . . . . . .

3.22 Kinematics coverage of the four resonance measurements (colored con-
tours), together with the DIS kinematics (black contours). . . . . . .

3.23 Kinematics of the beam normal asymmetry background. The incident and the scattered electrons' momenta are $\vec{k}_{e}$ and $\vec{k}_{e}^{\prime}$, and $\vec{S}_{V, H, L}$ denote respectively the incident electron's spin polarization components in the vertical, horizontal, and longitudinal directions. The central scattering angle of the spectrometer is $\theta_{0}$ and the scattered electron's momentum has an out-of-plane angle denoted by $\theta_{t r} \ldots$. . . . . . . . . . 
3.24 ADC spectra of the HAPPEX detector for two runs of the dipole scan [15]. This detector was a kind of calorimeter and recorded the energy deposited. The spectra for the elastic events is labeled "High Energy Electrons". The filled histogram is for the example of a detune by $14 \%$, indicating that the rescattering background is generally formed by lower energy particles. The approximate threshold for the trigger in this study is shown. The threshold for a high-energy electron that corresponds to a PVDIS trigger would be at approximately channel 900 in this spectrum, leading to further suppressions of the background. . . . . . . . . . . . . . . . .

3.25 The function $P_{\mathrm{rs}}(E)$ determined from HAPPEX data [15]. It is the product of the probability to re-scatter in the HRS and the probability for that particle to cause an electron trigger. Although it wasn't measured symmetrically about zero, we've assumed this symmetry here.138

4.1 $W$-dependence of the parity-violating asymmetries in $\vec{e}-{ }^{2} \mathrm{H}$ scattering extracted from this experiment. The physics asymmetry results $A_{P V}^{\text {phys }}$ for the four kinematics I, II, III and IV (solid circles, solid squares, solid triangles, and open triangles, respectively), in ppm, are scaled by $1 / Q^{2}$ and compared with calculations from Ref. [85] (theory $A$, dashed lines), Ref. [86] (theory $B$, dotted lines), Ref. [87] (theory $C$, solid lines) and the DIS estimation (dash-double-dotted lines) using Equations $(1.2 .4,1.2 .7,1.2 .8)$ with the extrapolated CJ PDF [89]. The vertical error bars for the data are statistical uncertainties, while the horizontal error bars indicate the root-mean-square values of the $W$ coverage of each bin. The experimental systematic uncertainties are shown as the shaded bands at the bottom. For each of the four kinematics, calculations were performed at the fixed $E_{b}$ and $Q^{2}$ values of Table 4.1 and with a variation in $W$ to match the coverage of the data. Theories $B$ and $C$ each have three curves showing the central values and the upper and lower bounds of the calculation. Uncertainties of the DIS calculation were below 1 ppm and are not visible. . . . . . .

4.2 Results on quark weak axial coupling combination $2 C_{2 u}-C_{2 d}$ from this measurement, shown as a function of $C_{2 u}+C_{2 d}$ v.s. $C_{2 u}-C_{2 d}$. The result is compared with previous measurements from SLAC (wide shaded band) [4, 5], SAMPLE (the two vertical shaded bands) [82], the Particle Data Group best fit (red ellipse) [12], and the Standard Model prediction (green solid circle). The SLAC results are shown with the same assumption as this experiment that the quark weak vector couplings $C_{1 q}$ agree with the Standard Model. . . . . . . . . . . . . . 153 
4.3 Correlation between the higher twist coefficient and $2 C_{2 u}-C_{2 d}$ from asymmetry results of $Q^{2}=1.085$ and $1.901(\mathrm{GeV} / \mathrm{c})^{2}$ (red ellipse), compared with the value of $2 C_{2 u}-C_{2 d}$ assuming $\beta_{H T}=0$ (blue band) and the Standard Model prediction (green star). . . . . . . . . . . . 155 


\section{List of Tables}

2.1 Position, material, and thickness for the target system used in this experiment. The position is defined along the beam direction with respect to the hall center. . . . . . . . . . . . . . . . . . . 39

2.2 Main design characteristics of the Hall A High Resolution Spectrometers. 45

3.1 Overview of kinematic settings of the experiment and the observed scattered electron rate $R_{e}$ and the negatively charged pion to electron rate ratio $R_{\pi} / R_{e}$. The kinematics include the beam energy $E_{b}$, and the spectrometer central angle $\theta_{0}$ and central momentum $E_{0}^{\prime}$. Measurement of the transverse asymmetry $A_{n}$ was performed at the production DIS settings on December 1-2. . . . . . . . . . . . . .

3.2 Corrections to asymmetries evaluated using the dithering method, $\Delta A_{\text {dit }}$. The " $\mathrm{n}$ " and "w" means "narrow" and "wide", and they refer to the DAQ trigger type (Section 2.6.2). The corrections were applied as $A_{\text {dit }}^{\text {bcraw }}=A^{\text {raw }}-\Delta A_{\text {dit }}[$ Equation 3.4.1] . . . . . . . . . . .

3.3 Corrections to the measured raw asymmetries from the narrow and the wide triggers due to beam energy and position changes using the dithering and the regression methods. The asymmetry errors shown are statistical only. The differences between the two corrected asymmetries, $\left|A_{\text {dit }}^{\text {bc,raw }}-A_{\text {reg }}^{\text {bc,raw }}\right|$, were used as the uncertainty due to beam corrections. . . . . . . . . . . . . . . . .

3.4 Average beam polarization $P_{b}$ for each kinematics. These are either the combined results of Compton and Møller measurements, or results from Møller alone, depending on which polarimeter was available during the corresponding run period. For DIS\#1 and \#2 the corrections were applied run-by-run and the statistically-averaged value of $P_{b}$ is shown. For resonance kinematics, each setting had short running period and a single value was used for all runs of each period. . . . . . . . . . .

3.5 Simulated DAQ deadtime loss for all kinematics and for both narrow (n) and wide (w) paths, along with the fractional contributions from Group, VETO and OR deadtimes. . . . . . . . . . . . . . . .

3.6 PVDIS $\mathrm{Q}^{2}$ uncertainty due to optics calibration. For each HRS, the kinematics are shown from left to right in the chronological order. . . 108 
3.7 Comparison of $Q^{2}, x$, and $W^{2}$ between HAMC and data for all kinematics. The Left and the Right DIS\#2 have been combined. . . . . . 111

3.8 Beam depolarization correction $\bar{f}_{\text {depol }}$ for all kinematics. . . . . . . . 115

3.9 Radiative correction factors. For each kinematics, the simulated asymmetries using two resonance models are shown. In kinematic regions where the resonance models are not available, the cross-section (c.s.)scaling model was used. These asymmetries were input to Eq. (3.11.9) to obtain the radiative correction factors. Results from the two models were averaged to provide the final correction $1+\bar{f}_{r c}$, and the difference between the two was combined with uncertainties of resonance models themselves to provide the total uncertainty on $\bar{f}_{r c} \ldots \ldots \ldots$

3.10 For DIS kinematics: Beam-corrected pion asymmetries $A_{\pi, \text { dit }}^{\text {bc,raw }}$ with their statistical uncertainties, electron contamination in the pion triggers $f_{e / \pi}$, pion asymmetries results after being corrected for electron contamination $A_{\pi}^{\text {phys }}$, pion contamination in electron triggers $f_{\pi / e}$, and total uncertainties on the electron asymmetry results due to pion background. . . . . . . . . . . . . . . .

3.11 For resonance kinematics: Beam-corrected pion asymmetries $A_{\pi, \text { dit }}^{\text {bc,raw }}$ with their statistical uncertainty, electron contamination in the pion triggers $f_{e / \pi}$, pion asymmetries results after being corrected for electron contamination $A_{\pi}^{\text {phys }}$, pion contamination in electron triggers $f_{\pi / e}$, and total uncertainties on the electron asymmetry results due to the pion

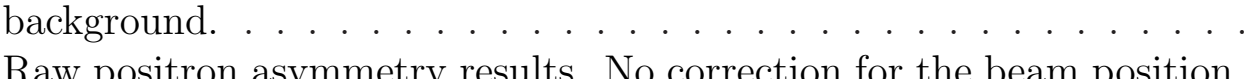

3.12 Raw positron asymmetry results. No correction for the beam position, energy, and polarization, or the $\pi^{+}$background was made.

3.13 Results for positron contamination $f_{e^{+} / e^{-}}$and its statistical uncertainty, and the total uncertainty on electron asymmetry due to pair production background. Only DIS kinematics were evaluated. The errors shown for $f_{e^{+} / e^{-}}$are statistical only, and a $10 \%$ systematic uncertainty on $f_{e^{+}} / e^{-}$was used in the evaluation of $\frac{\Delta A_{e}}{A_{e}} \ldots \ldots \ldots$

3.14 Relative differences between calculated $\mathrm{Al}$ and $\mathrm{D}_{2}$ asymmetries, $\left(A_{\mathrm{Al}}-\right.$ $\left.A_{D}\right) / A_{D}$, the fractional event rate from $\mathrm{Al}$ endcaps $\alpha_{\mathrm{Al} / \mathrm{D}}$, corrections applied to measured electron asymmetries $\bar{f}_{\mathrm{Al}}$ using Eq. (3.12.9), and the relative uncertainty in the corrected electron asymmetry due to endcap corrections $\left(\Delta A_{e} / A_{e}\right)_{\mathrm{Al}} \ldots \ldots \ldots \ldots \ldots$

3.15 Results from the dedicated beam transverse asymmetry measurements and estimation of the total uncertainty on the PVDIS electron asymmetry due to beam transverse polarization. The dithering-corrected values were used for both $A_{e}^{\text {meas }}$ and $A_{n}^{\text {meas }}$. For DIS\#2, the electron asymmetry is the combined value from the Left and the Right HRS. 
4.1 Parity violating asymmetry results for the resonance kinematics. See text for details. . . . . . . . . . . . . . . . . . .

4.2 Group asymmetry results for the resonance measurements. The $W$ and $Q^{2}$ values are averaged over the acceptance of each group. $A_{\text {phys }}$ has all systematic corrections applied. The only corrections that are groupspecific are the radiative correction $\bar{f}_{\mathrm{rc}}$ and the deadtime correction $\bar{f}_{\mathrm{dt}}$, which are given in Table 3.16. Other corrections are the same as those for global triggers shown in Table 4.1 . . . . . . . . . . . . . . 146

4.3 Parity violating asymmetry results for the DIS kinematics. See text of Section 4.1 for details. . . . . . . . . . . . . . . . . . . . . . . . . . . 149

4.4 Comparison of asymmetry calculation using different structure functions. Values for $\alpha_{E M}\left(Q^{2}\right)$ were calculated using $\alpha_{E M}\left(Q^{2}=0\right)=$ $1 / 137.036$ and $C_{1 q, 2 q}^{\mathrm{SM}}\left(Q^{2}\right)$ were based on Table 7 and Eq. $(114-115)$ of Ref. [88]. . . . . . . . . . . . . . . . . . . .

4.5 Results of the quark weak axial coupling combination $2 C_{2 u}-C_{2 d}$ extracted from the asymmetries measured in this experiment. The corresponding Standard Model predicted values are also shown for comparison.152 


\section{1}

\section{Introduction}

Parity symmetry, sometimes referred to as mirror image symmetry, implies that flipping left and right does not change the laws of physics. While violation of parity symmetry in the weak nuclear force was discovered in $1957[1,2,3]$, the appropriate theory to describe such phenomenon was not established until the first parity violation experiment using an electron beam scattered off a deuterium target was completed in 1978 by American physicist Charles Prescott [4, 5]. This experiment showed that the $S U(2)_{L} \times U(1)_{Y}$ group mixing theory, first proposed by Glashow in 1961 [6] and then modified by Weinberg [7] and Salam [8] separately in 1967 and 1968, to be the most promising theory to describe both electromagnetic and weak interactions [9]. This electro-weak unification has since become a staple of the Standard Model of particle physics.

Some people are left-handed and most are right-handed. Similarly, elementary particles have a handedness (called helicity) as well: A particle is called right-handed if its spin is in the same direction as its motion, and left-handed if they are the opposite. While particles can be equally probable to be in both helicity states, they could behave

differently: In the language of quantum field theory, all spin-1/2 elementary particles 
(elementary fermions) carry two types of couplings: axial and vector. The axial coupling describes the difference in the strength of how an elementary fermion, when in its left- and right-handed states, interact with the force mediating bosons; while the vector coupling describes the average strength of the two helicity states. For example, for electromagnetic interactions all particles carry only the vector coupling - the electric charge - which means they are ambidexterious, i.e. with equal electromagnetic strength regardless of their helicity.

But the weak interaction is different. According to the Weinberg-Salam-Glashow theory, the charged weak interaction carried by $W^{ \pm}$bosons interact only with lefthanded fermions. For neutral weak interactions carried by the $Z^{0}$ boson, both left- and right-handed fermions participate but there is a difference in their coupling strength. Since calculations of physical observables require multiplying the couplings of the two fermions involved, it is the coexistence of both vector and axial weak couplings that causes parity violation in neutral weak interactions.

In the Standard Model, the weak axial coupling $g_{A}$ of a fermion equals to its weak isospin $T_{3}: g_{A}^{f}=T_{3}=1 / 2$ for up, charm and top quarks and $=-1 / 2$ for electrons, down, strange and bottom quarks. The weak vector coupling $g_{V}$ is related to the particle's $T_{3}$ as well as its electric charge $Q: g_{V}^{f}=T_{3}-2 Q \sin ^{2} \theta_{W}$ with the weak mixing angle $\sin ^{2} \theta_{W}=0.23115$. Precision measurements of both the weak vector and axial couplings of all elementary particles - electrons, muons, quarks, etc. - and the consistency among measurements will shed light on how well the Standard Model works. So far we have not found any clear indications that the Standard Model needs to be amended, in particular with the latest results on Higgs searches at the Large Hadron Collider [10, 11], but the majority of physicists believe an extension of the Standard Model will become inevitable when experiments are performed at higher energies or with better precision in the future, if not now. Any inconsistency among 
experimental results on the weak couplings and comparison with the Standard Model will tell us in which direction we should look for physics not included in the Standard Model.

While results from parity violation experiments have clearly established the nonzero weak axial coupling of electrons [12], direct data on the quark weak axial coupling are scarce. The experiment results from this PVDIS experiment are about the change this picture.

\subsection{Deep Inelastic Electron Scattering}

The PVDIS experiment employs the deep inelastic electron scattering as a probe to study the parity violation in weak interactions. A brief review of the process is presented in this section.

\subsubsection{Electron Scattering}

In the most simplified picture of electron scattering, the electron interacts with a target through the electromagnetic force, as shown in Figure 1.1. A virtual photon with four momentum $q=(\nu, \bar{q})$ is exchanged between the electron and the target nucleus (or nucleon). In the case of scattering with a fixed target in the lab frame, the target has an initial four momentum $P=(M, 0)$ and its final state varies depending on the kinematics: the same as the original nucleus - elastic; nucleus broken up into nucleons - quasi elastic; bound excited states of the nucleon - nucleon resonances; or fragments of the nucleon - DIS. For inclusive measurement, only the scattered electron is detected. If we denote the initial and final momenta of the electron by $k=$ $(E, \bar{k})$ and $k^{\prime}=\left(E^{\prime}, \bar{k}^{\prime}\right)$, the four momentum of the exchanged photon is determined as $q=k-k^{\prime}=(\nu, \bar{q})$. The virtual photon energy $\nu=E-E^{\prime}$ is also called the "energy 
loss" of the electron.

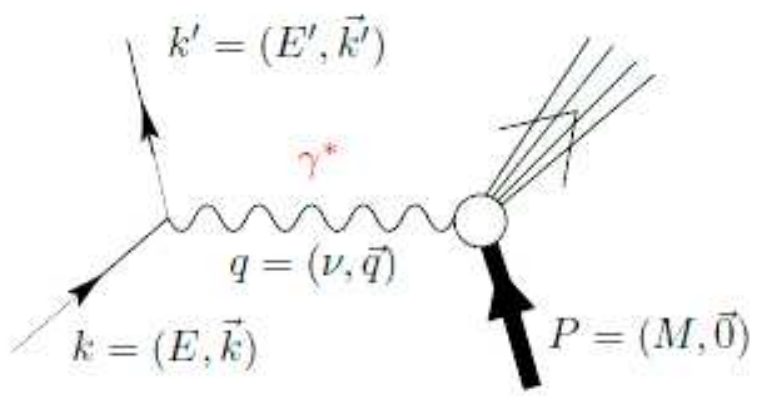

Figure 1.1: Feynman diagram of electron scattering through one-photon exchange.

We can define some relativistic invariants characterizing the reaction:

- The virtuality of the exchanged photon is defined as the negative of its four momentum squared $Q^{2} \equiv-\left(k-k^{\prime}\right)^{2}=-q^{2}$. For fixed target scattering, the $Q^{2}$ can be calculated as

$$
Q^{2}=2 E E^{\prime}(1-\cos \theta)
$$

where $\theta$ is the scattering angle of the electron in the lab frame. For real photons, $Q^{2}=0$.

- The invariant mass is defined as

$$
W^{2} \equiv(P+q)^{2}=M^{2}+2 M \nu-Q^{2}
$$

where $M$ is the mass of the target nucleus in the case of elastic scattering, and the mass of nucleon in other cases as will be described later.

- The Bjorken scaling variable is defined as

$$
x_{b j} \equiv \frac{Q^{2}}{2 P \cdot q}=\frac{Q^{2}}{2 M \nu}
$$


This invariant variable is most useful in describing the deep inelastic scattering (DIS) process, which will be explained later.

The cross section for electron scattering depends on the variables $Q^{2}$ and $\nu$. As $Q^{2}$ and $\nu$ vary, the target goes through different excitation states, and the corresponding cross sections exhibit different characteristics, based on which we can categorize the scattering process into the follow regions:

- Elastic scattering: At low $Q^{2}$ and $\nu$, the distance probed by the process are not small enough to reveal the internal structure of the target. The target nucleus appears to be a rigid object and reacts coherently during the scattering. The momentum transfer is shared among all components of the nucleus, and the energy loss is $\nu=Q^{2} / 2 M_{t}$ with $M_{t}$ the mass of the target nucleus.

- Quasi-elastic scattering: As the distance probed gets smaller, the virtual photon starts to see the composing nucleons of the target nucleus. When the energy loss $\nu$ is larger than the nuclear binding energy, the nucleus is broken up and the nucleons no longer react coherently. We can then picture the process as the electron scattering elastically from a nucleon, therefore the name "quasi-elastic scattering". Kinematically, quasi-elastic scattering happens at $\nu=Q^{2} / 2 M_{N}$ with $M_{N}$ the nucleon mass, and $W^{2}=M_{N}^{2}+2 M_{N} \nu-Q^{2}=M_{N}^{2}$. The threshold to separate the elastic and the quasi-elastic scatterings is the nuclear binding energy. Unlike the elastic scattering, the target nucleons for quasi-elastic scattering are not at rest in the lab frame. They typically have momenta of $\sim 200$ $\mathrm{MeV} / \mathrm{c}$ due to their "Fermi motion" within the parent nucleus. This "Fermi motion" causes a broadening of the quasi-elastic peak.

- Resonances: As $Q^{2}$ and $\nu$ increase we enter the resonance region. In this region the nucleons absorb the virtual photons and get excited into higher energy 
states, or the "resonances". The $W$ value of nucleon resonances spans from $M_{N}$ to $2 \mathrm{GeV}$. These resonance states then decay mainly by emitting mesons, so a significant pion electroproduction is commonly seen in the resonance region. The first resonance is the $\Delta(1232)\left(M_{\Delta}=1232 \mathrm{MeV}\right)$, which is usually the only one unambiguously visible in inclusive experiments. Beyond $W \sim 1.4 \mathrm{GeV}$ the resonance peaks overlap with tails from nearby resonances and can not be clearly distinguished.

- Deep inelastic scattering: As $Q^{2}$ and $\nu$ increase further we reach the last domain of the scattering process, which is the deep inelastic scattering (DIS) region. The DIS is traditionally defined as $W>2 \mathrm{GeV}$. In the DIS region, the distance probed by the high energy virtual photon is small enough that the constituents of the nucleon are resolved. Therefore, DIS can be interpreted as scattering off asymptotically free quarks and antiquarks. Usually DIS is treated in an inertial frame where the nucleon moves with infinite momentum along the direction of $\vec{q}$. In this frame, the Bjorken variable $x_{b j}$ is the fraction of the nucleon's momentum carried by the struck quark or antiquark and the DIS formalism takes the simplest form.

For the PVDIS experiment, as mentioned earlier, we aim to test the Standard Model from the aspect of weak axial couplings of the quarks. Therefore, direct scattering from the quarks is the appropriate probe and DIS is obviously the region of interest. 


\subsubsection{Unpolarized DIS Cross Section and Structure Func- tions}

To consider the unpolarized DIS cross section, we start with the simplest case of an electron scattering off a point-like, spin-0, infinitely heavy target. The cross section of this simple process is called the Mott cross section, which is given by

$$
\left(\frac{d^{2} \sigma}{d \Omega}\right)_{\mathrm{Mott}}=\frac{\alpha^{2} \cos ^{2} \frac{\theta}{2}}{4 E^{2} \sin ^{4} \frac{\theta}{2}}
$$

where $\alpha$ is the fine structure constant and $\theta$ is the scattering angle. In reality, the target is never point-like. Two unpolarized structure functions, conventionally written as $W_{1}\left(\nu, Q^{2}\right)$ and $W_{2}\left(\nu, Q^{2}\right)$, are used to describe the spatial extension of the target, and the unpolarized DIS cross section is written as

$$
\frac{d^{2} \sigma}{d \Omega d E^{\prime}}=\left(\frac{d^{2} \sigma}{d \Omega}\right)_{\mathrm{Mott}}\left[W_{2}\left(\nu, Q^{2}\right)+2 W_{1}\left(\nu, Q^{2}\right) \tan ^{2} \frac{\theta}{2}\right]
$$

We customly express the structure functions $W_{1}$ and $W_{2}$ in terms of two other unpolarized scaling structure functions $F_{1}\left(x, Q^{2}\right)$ and $F_{2}\left(x, Q^{2}\right)$ :

$$
\begin{aligned}
& W_{1}\left(\nu, Q^{2}\right) \equiv \frac{F_{1}\left(x, Q^{2}\right)}{M} \\
& W_{2}\left(\nu, Q^{2}\right) \equiv \frac{F_{2}\left(x, Q^{2}\right)}{\nu}
\end{aligned}
$$

The two structure functions $F_{1}\left(x, Q^{2}\right)$ and $F_{2}\left(x, Q^{2}\right)$ are related by

$$
F_{1}\left(x, Q^{2}\right)=\frac{F_{2}\left(x, Q^{2}\right)\left(1+\gamma^{2}\right)}{2 x\left(1+R\left(x, Q^{2}\right)\right)}
$$


where $\gamma^{2} \equiv \frac{Q^{2}}{\nu^{2}}$, and $R \equiv \frac{\sigma_{L}}{\sigma_{T}}$ is the ratio of longitudinal to transverse virtual photon electromagnetic cross sections. The unpolarized DIS cross section can then be written as

$$
\frac{d^{2} \sigma}{d \Omega d E^{\prime}}=\left(\frac{d^{2} \sigma}{d \Omega}\right)_{\mathrm{Mott}}\left[\frac{1}{\nu} F_{2}\left(x, Q^{2}\right)+\frac{2}{M} F_{1}\left(x, Q^{2}\right) \tan ^{2} \frac{\theta}{2}\right] .
$$

One interesting phenomenon about the structure functions is their scaling behavior. Bjorken first pointed out [13] that if the nucleon is made of point-like spin- $1 / 2$ constituents, then in the limit where $Q^{2} \rightarrow \infty$ and $\nu \rightarrow \infty$ with $x$ fixed, the structure functions are independent of $Q^{2}$ and can be written as $F_{1}(x)$ and $F_{2}(x)$. Furthermore, $\sigma_{L}$ vanishes in this limit. So Equation 1.1 .8 simplifies into

$$
F_{2}(x)=2 x F_{1}(x)
$$

which is know as the Callan-Gross relation.

\subsubsection{The Quark Parton Model}

The scaling behavior of the structure functions in the Bjorken limit is based on the fact that the nucleon must be made of point-like spin- $1 / 2$ constituents. This implication leads to the quark-parton model (QPM), which describes the nucleon as a sum of free partons (quarks, antiquarks and gluons). Each of the partons carries a fraction $x$ of the nucleon's longitudinal momentum and reacts to the virtual photon probe independently. Within this description, the cross section of the nucleon is just the sum of the cross sections from its composing partons, properly weighted by their 
occurring probabilities. Therefore the structure function $F_{1}(x)$ can be expressed as

$$
\begin{aligned}
F_{1}(x) & =\frac{1}{2} \sum Q_{i}^{2}\left[q_{i+}(x)+q_{i-}(x)+\bar{q}_{i+}(x)+\bar{q}_{i-}(x)\right] \\
& =\frac{1}{2} \sum Q_{i}^{2}\left[q_{i}(x)+\bar{q}_{i}(x)\right]
\end{aligned}
$$

where $i$ is quark flavour, $Q_{i}$ is the corresponding quark electric charge, $q_{i+}(x)\left[q_{i-}(x)\right]$ is the probability that the $i^{\text {th }}$ quark with its spin aligned parallel (anti-parallel) to the nucleon spin carries the fraction $x$ of the nucleon's momentum, and $q_{i}(x)=$ $q_{i+}(x)+q_{i-}(x)$ is the overall probability of the $i^{\text {th }}$ quark carrying the fraction $x$ of the nucleon's momentum. The $q_{i}(x)$ is also defined as the unpolarized parton distribution function (PDF).

\subsection{PVDIS Formalism}
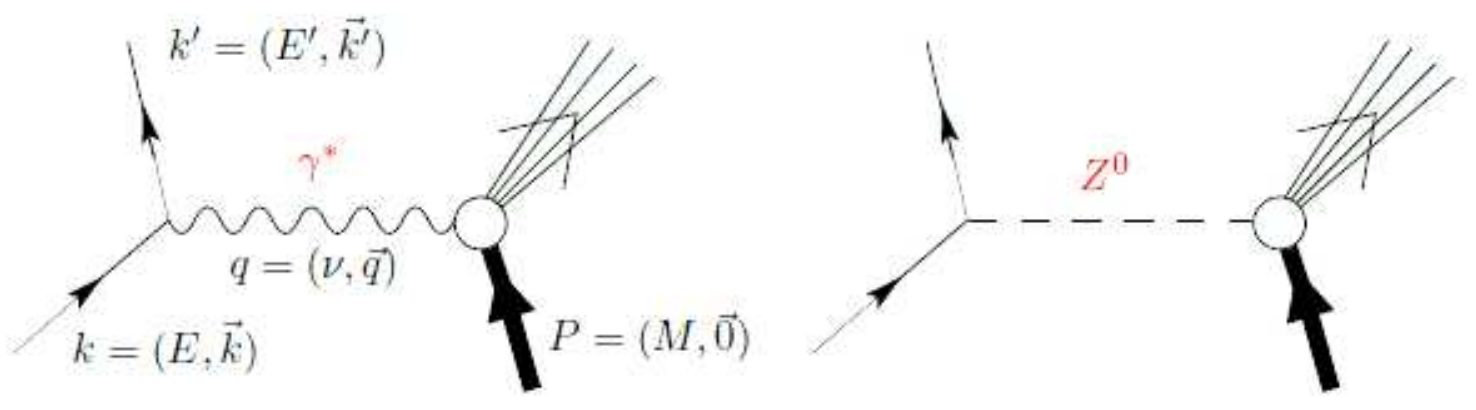

Figure 1.2: Feynman diagram of electron scattering including both electromagnetic and weak interactions.

Now we invite the weak interaction into the picture. In addition to the exchange of a photon $\gamma$, the electron can also interact with the target through weak interaction by exchanging a $Z^{0}$ boson, as shown in Figure 1.2. While direct measurement of the $Z^{0}$ exchange alone is difficult, the interference between $\gamma$ and $Z^{0}$ exchanges is 
parity-violating and can be accessed by taking the relative difference in scattering cross sections between left- and right-handed electrons scattering off an unpolarized target. Measurement of parity violation in electron scattering has been widely used in the past decade to access the contribution from strange quark sea to the nucleon form factors $[14,15,16,17,18,19]$, and to study heavy nuclear matter in order to look into the internal structure of neutron stars [20]. In these experiments, the energy and direction of the scattered electrons are carefully selected such that the target does not break (elastic scattering) and the aforementioned properties can be extracted from data. The weak axial couplings of quarks, on the other hand, can be measured better if the nucleon is broken into quarks. Therefore, DIS offers a unique window for this study.

For electron scattering processes, the scattering amplitude is the product of currents for the electron and the hadron, sandwiching around the photon and the $Z^{0}$ propagator. At $Q^{2} \ll M_{Z}^{2}$, the amplitudes are:

$$
\mathcal{M}_{\gamma}=j_{\mu}\left(\frac{1}{q^{2}}\right) J^{\mu}, \quad \mathcal{M}_{Z}=j_{\mu}\left(\frac{1}{M_{Z}^{2}}\right) J^{\mu}
$$

With a longitudinally polarized electron beam, the cross sections for scattering rightand left-handed electrons off an unpolarized target is proportional to the square of the total amplitudes:

$$
\sigma^{r} \propto\left(\mathcal{M}_{\gamma}+\mathcal{M}_{Z}^{r}\right)^{2}, \quad \sigma^{l} \propto\left(\mathcal{M}_{\gamma}+\mathcal{M}_{Z}^{l}\right)^{2}
$$

where $\mathcal{N}_{Z}^{r}$ and $\mathcal{M}_{Z}^{l}$ represent the amplitudes for incident right- and left-handed electrons, respectively. The parity-violating asymmetry describes the relative difference between scattering cross sections with right-handed electrons $\sigma_{R}$ and that with left- 
handed electrons $\sigma_{L}$ :

$$
A_{P V} \equiv \frac{\sigma_{R}-\sigma_{L}}{\sigma_{R}+\sigma_{L}}=\frac{\left(\mathcal{M}_{\gamma}+\mathcal{M}_{Z}^{r}\right)^{2}-\left(\mathcal{M}_{\gamma}+\mathcal{M}_{Z}^{l}\right)^{2}}{\left(\mathcal{M}_{\gamma}+\mathcal{M}_{Z}^{r}\right)^{2}+\left(\mathcal{M}_{\gamma}+\mathcal{M}_{Z}^{l}\right)^{2}} \sim \frac{\mathcal{M}_{Z}^{r}-\mathcal{M}_{Z}^{l}}{\mathcal{M}_{\gamma}}
$$

In the specific case of DIS, the parity violating asymmetry can be written as [21]

$$
A_{P V}=-\frac{G_{F} Q^{2}}{4 \sqrt{2} \pi \alpha}\left[a_{1}(x) Y_{1}(y)+a_{3}(x) Y_{3}(y)\right]
$$

where $G_{F}$ is the Fermi constant, $\alpha$ is the fine structure constant, and $Q^{2} \equiv-q^{2}$ is defined in Section 1.1.1. E and $E^{\prime}$ are the incident and the scattered electron's energy, respectively. The kinematic factors $Y_{1,3}$ are

$$
Y_{1}=\left[\frac{1+R^{\gamma Z}}{1+R^{\gamma}}\right] \frac{1+(1-y)^{2}-y^{2}\left[1-\frac{r^{2}}{1+R^{\gamma Z}}\right]-x y \frac{M}{E}}{1+(1-y)^{2}-y^{2}\left[1-\frac{r^{2}}{1+R^{\gamma}}\right]-x y \frac{M}{E}}
$$

and

$$
Y_{3}=\left[\frac{r^{2}}{1+R^{\gamma}}\right] \frac{1-(1-y)^{2}}{1+(1-y)^{2}-y^{2}\left[1-\frac{r^{2}}{1+R^{\gamma}}\right]-x y \frac{M}{E}},
$$

where $x$ is the Bjorken scaling variable, $M$ is the nucleon mass and $\nu=E-E^{\prime}$ the energy transfer from the electron to the target; $y=\nu / E=\left(E-E^{\prime}\right) / E$ is the fractional energy loss of the electron, $r^{2}=1+\frac{Q^{2}}{\nu^{2}}$, and $R^{\gamma(\gamma Z)}$ is the ratio of the longitudinal to transverse virtual photon electromagnetic absorption cross sections $\left(\gamma-Z^{0}\right.$ interference cross sections). To a good approximation one has $R^{\gamma} \approx R^{\gamma Z}$ and 
$Y_{1}(y) \approx 1$. The $a_{1,3}$ terms are

$$
\begin{aligned}
& a_{1}(x)=2 g_{A}^{e} \frac{F_{1}^{\gamma Z}}{F_{1}^{\gamma}}, \\
& a_{3}(x)=g_{V}^{e} \frac{F_{3}^{\gamma Z}}{F_{1}^{\gamma}},
\end{aligned}
$$

where $g_{V, A}^{e}$ are respectively the electron's vector and axial couplings to the $Z^{0}$. The structure functions of the target, $F_{1,3}^{\gamma, \gamma Z}$, can be interpreted in the QPM as being related to the PDFs $q_{i}(x)$ and $\bar{q}_{i}(x)$ of the target:

$$
\begin{aligned}
F_{1}^{\gamma}(x) & =\frac{1}{2} \sum Q_{i}^{2}\left[q_{i}(x)+\bar{q}_{i}(x)\right], \\
F_{1}^{\gamma Z}(x) & =\sum Q_{i} g_{V}^{i}\left[q(x)+\bar{q}_{i}(x)\right], \\
F_{3}^{\gamma Z}(x) & =2 \sum Q_{i} g_{A}^{i}\left[q_{i}(x)-\bar{q}_{i}(x)\right] .
\end{aligned}
$$

Here the summation is over the quark flavor $i=u, d, s \cdots$ and $Q_{i}$ is the corresponding quark electric charge. In most world parameterizations, it is common to fit the structure function $F_{2}$ and $R$ simultaneously to cross section data, where $F_{2}$ is related to $F_{1}$ as

$$
F_{2}^{\gamma(\gamma Z)}=\frac{2 x F_{1}^{\gamma(\gamma Z)}\left(1+R^{\gamma(\gamma Z)}\right)}{r^{2}}
$$

or equivalently:

$$
F_{1}^{\gamma(\gamma Z)}=\frac{r^{2} F_{2}^{\gamma(\gamma Z)}}{2 x\left(1+R^{\gamma(\gamma Z)}\right)} .
$$

In the QPM the ratio $R^{\gamma(\gamma Z)}$ is treated as zero and one can construct the $F_{1,2}$ structure 
functions as

$$
\begin{aligned}
F_{2}^{\gamma}(x) & =2 x F_{1}^{\gamma}(x)=x \sum Q_{i}^{2}\left[q_{i}(x)+\bar{q}_{i}(x)\right] \\
F_{2}^{\gamma Z}(x) & =2 x F_{1}^{\gamma Z}(x)=2 x \sum Q_{i} g_{V}^{i}\left[q_{i}(x)+\bar{q}_{i}(x)\right] .
\end{aligned}
$$

For electron scattering, it is traditional to define the product of the electron and the quark weak couplings as the effective weak coupling constants $C_{1 q, 2 q}$ :

$$
\begin{array}{cc}
C_{1 u}=2 g_{A}^{e} g_{V}^{u}, & C_{2 u}=2 g_{V}^{e} g_{A}^{u} \\
C_{1 d}=2 g_{A}^{e} g_{V}^{d}, & C_{2 d}=2 g_{V}^{e} g_{A}^{d} .
\end{array}
$$

If considering only the leading process of one-photon and one- $Z^{0}$ exchanges between the electron and the target, these are related to the weak mixing angle $\theta_{w}$ as

$$
\begin{aligned}
& C_{1 u}=2 g_{A}^{e} g_{V}^{u}=2\left(-\frac{1}{2}\right)\left(\frac{1}{2}-\frac{4}{3} \sin ^{2} \theta_{W}\right)=-\frac{1}{2}+\frac{4}{3} \sin ^{2} \theta_{W}, \\
& C_{2 u}=2 g_{V}^{e} g_{A}^{u}=2\left(-\frac{1}{2}+2 \sin ^{2} \theta_{W}\right)\left(\frac{1}{2}\right)=-\frac{1}{2}+2 \sin ^{2} \theta_{W}, \\
& C_{1 d}=2 g_{A}^{e} g_{V}^{d}=2\left(-\frac{1}{2}\right)\left(-\frac{1}{2}+\frac{2}{3} \sin ^{2} \theta_{W}\right)=\frac{1}{2}-\frac{2}{3} \sin ^{2} \theta_{W} \\
& C_{2 d}=2 g_{V}^{e} g_{A}^{d}=2\left(-\frac{1}{2}+2 \sin ^{2} \theta_{W}\right)\left(-\frac{1}{2}\right)=\frac{1}{2}-2 \sin ^{2} \theta_{W} .
\end{aligned}
$$

Defining $q_{i}^{ \pm}(x) \equiv q_{i}(x) \pm \bar{q}_{i}(x)$, one has in the QPM

$$
\begin{aligned}
& a_{1}(x)=2 \frac{\sum C_{1 i} Q_{i} q_{i}^{+}(x)}{\sum Q_{i}^{2} q_{i}^{+}(x)}, \\
& a_{3}(x)=2 \frac{\sum C_{2 i} Q_{i} q_{i}^{-}(x)}{\sum Q_{i}^{2} q_{i}^{+}(x)} .
\end{aligned}
$$

For an isoscalar target such as the deuteron, neglecting effects from heavier quark flavors and assuming the isospin symmetry that $u^{p}=d^{n}, d^{p}=u^{n}\left[u, d^{p(n)}\right.$ are the up 
and down quark PDF in the proton (neutron)], $s=\bar{s}$, and $c=\bar{c}$, the functions $a_{1,3}(x)$ simplify to

$$
\begin{aligned}
& a_{1}(x)=\frac{6\left[2 C_{1 u}\left(1+R_{c}\right)-C_{1 d}\left(1+R_{s}\right)\right]}{5+R_{s}+4 R_{c}}, \\
& a_{3}(x)=\frac{6\left(2 C_{2 u}-C_{2 d}\right) R_{v}}{5+R_{s}+4 R_{c}}
\end{aligned}
$$

where $R_{c} \equiv[2(c+\bar{c})] /(u+\bar{u}+d+\bar{d}), R_{s} \equiv[2(s+\bar{s})] /(u+\bar{u}+d+\bar{d})$ and $R_{V} \equiv$ $(u-\bar{u}+d-\bar{d}) /(u+\bar{u}+d+\bar{d})$. The asymmetry then becomes

$$
A_{P V}=\left(\frac{3 G_{F} Q^{2}}{2 \sqrt{2} \pi \alpha}\right) \frac{2 C_{1 u}\left[1+R_{C}(x)\right]-C_{1 d}\left[1+R_{S}(x)\right]+Y_{3}\left(2 C_{2 u}-C_{2 d}\right) R_{V}(x)}{5+R_{S}(x)+4 R_{C}(x)}
$$

In addition, if one neglects sea quarks completely [22], $R_{c}=R_{s}=0, R_{v}=1$, no PDF is involved (i.e. neglecting nucleon structure) and

$$
a_{1}(x)=\frac{6}{5}\left(2 C_{1 u}-C_{1 d}\right), \quad a_{3}(x)=\frac{6}{5}\left(2 C_{2 u}-C_{2 d}\right)
$$

which lead to

$$
A_{P V}=\left(\frac{3 G_{F} Q^{2}}{10 \sqrt{2} \pi \alpha}\right)\left[\left(2 C_{1 u}-C_{1 d}\right)+Y_{3}\left(2 C_{2 u}-C_{2 d}\right)\right]
$$

Comparisons between Eq. (1.2.4) and Eq. (1.2.28) provides information on how much the input parton distribution functions affect the evaluation of the asymmetry. The magnitude of the asymmetry is in the order of $10^{-4}$, or $10^{2}$ parts per million (ppm) at $Q^{2}=1(\mathrm{GeV} / \mathrm{c})^{2}$. 


\subsection{Current knowledge on $C_{1 q}$ and $C_{2 q}$ Couplings}

The first parity violating electron scattering (PVES) experiment (SLAC E122) was carried out by C.Y. Prescott et al. $[4,5]$ more than three decades ago. During that experiment, a longitudinally polarized electron beam was scattered off an unpolarized deuteron target at three different beam energies (16.2 GeV, $19.4 \mathrm{GeV}$ and $22.2 \mathrm{GeV})$. The parity violating asymmetry was measured at 11 different kinematic settings, with $Q^{2}$ ranging from $0.92 \mathrm{GeV} / \mathrm{c}^{2}$ to $1.96 \mathrm{GeV} / \mathrm{c}^{2}$. Results from the experiment strongly favored the Weinberg-Salam-Glashow model, which was established later on as the electroweak unified theory in the Standard Model.

Subsequently in the past several decades, PVES has been a very useful tool used to study a wide range of physics, from searching for new physics beyond the Standard Model to investigating the structures of nuclei and nucleon $[14,15,16,17,18,19$, 20]. These PVES experiments, together with the complimentary measurements from atomic parity violation (APV) experiments [23], provide important input data from which the weak coupling constants $C_{1 q}$ and $C_{2 q}$ can be extracted and put into tests against the Standard Model. Figure 1.3 summarizes the current knowledge on $C_{1 q}$ and $C_{2 q}$ couplings.

As can be seen from Figure 1.3a, the constraints on the $C_{1 q}$ couplings are pretty good, due to the many experiments that provided input data for the extraction of $C_{1 q}$. The best results on $C_{1 q}$ up-to-date is provided by a combined fit using the latest APV Cesium result (the green band) and the world PVES data including the firststage result from QWeak (the blue ellipse) [24]. The $C_{1 q}$ couplings determined from this combined fit (the red ellipse) are in good agreement with the Standard Model predicted value (the black dot). The final result from QWeak, when released in the future, will have an improvement by a factor of five in the precision of the measured 


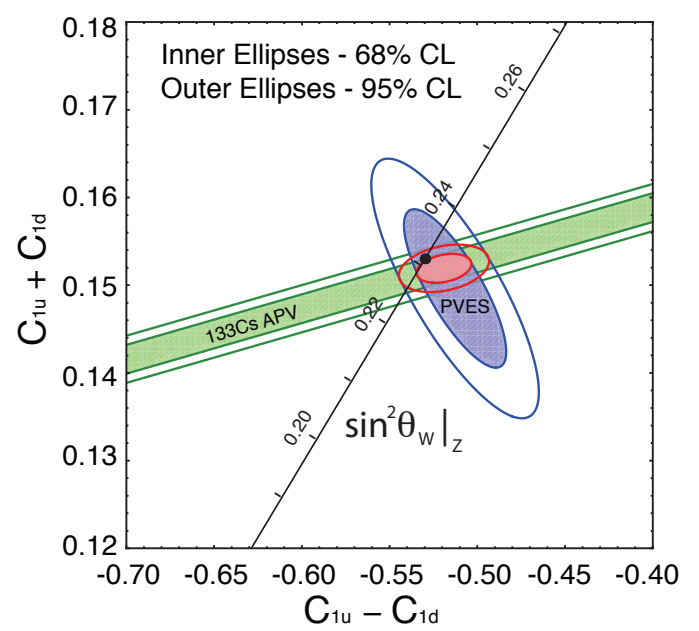

(a)

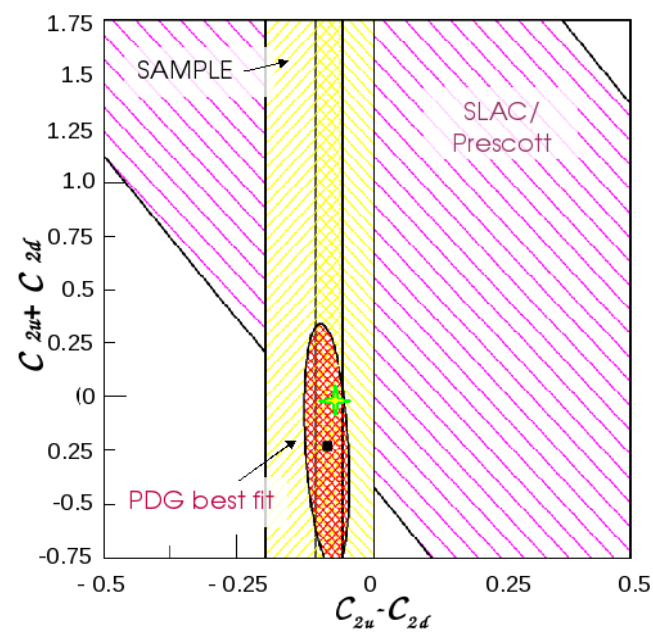

(b)

Figure 1.3: Existing constrains on the weak coupling constants $C_{1 q}$ and $C_{2 q}$. The $C_{1 q}$ plot (a) is from [24].

$A_{P V}$ (the projected final result of QWeak is shown as the thin blue band), which will put even better constraints on the $C_{1 q}$ couplings.

Compared with the current knowledge on $C_{1 q}$, our knowledge on the $C_{2 q}$ is rather limited, as shown in Figure 1.3b. All the PVES experiments after SLAC E122 were performed outside the DIS region, which could not provide clean extraction of $C_{2 q}$. So SLAC E122 is really the only one that directly measured $C_{2 q}$. But both its statistical uncertainty $(\sim 10 \%)$ and systematic uncertainty $(\sim 7 \%)$ were relatively large, due to the limitations of accelerator technology and experimental techniques at that time. Now more than three decades have passed, a new measurement of $A_{P V}$ in DIS with much smaller statistical and systematic uncertainties can be achieved, by taking advantages of both the high luminosity polarized electron beam available nowadays and the advancements in experimental techniques for controlling systematics in PVES experiments, which can significantly improve our knowledge on the $C_{2 q}$ couplings. Such a new measurement [25] has indeed been performed and is now reported in this thesis. 


\section{4 $A_{\mathrm{PV}}$ in the Resonance Region}

During the PVDIS experiment, the parity violating asymmetries $A_{P V}$ were also measured in the resonance region, but with much lower statistics that the DIS. The main purpose of these resonance measurements is to constrain the radiative corrections to the DIS measurements. But the resonance $A_{P V}$ is also a very interesting topic by itself. Measurements of the resonance $A_{P V}$ can help to test our understanding of the nucleon resonance structure. In the resonance region, the PV structure functions can be described in terms of longitudinal, transverse and axial PV response functions to specific resonance states, together with a non-resonant background. The first calculation of the resonance $A_{P V}$ was performed by Cahn and Gilman [21] on the first nucleon resonance, the $N \rightarrow \Delta(1232)$ transition. And more precise calculations in the resonance region have been performed subsequently [33]. However, these calculations remain largely unconstrained due to the lack of experimental data. The only existing measurement of resonance $A_{P V}$ is the $\Delta(1232)$ asymmetry from the proton reported by $G 0$ [34]. Therefore, the new measurements from the PVDIS experiment, which cover a wide range in the resonance region, play an important role in checking the reliability of those theoretical models for calculating the resonance $A_{P V}$. Such models are relevant for background corrections to other key PVES experiments [24].

Another remarkable feature in the resonance region is the so-called "quark-hadron duality". When first pointed out by Bloom and Gilman [26] for inclusive cross section measurements in the resonance region, the quark-hadron duality referred to the phenomenon that the averaged cross section over energy intervals of the resonance structures resemble those at asymptotically high energies. Over the past decade, duality has been verified in various areas of measurements, including the unpolarized structure functions $F_{2}$ and $F_{L}[27,28,29,30,31]$, the proton spin asymmetry $A_{1}^{p}$ [32], 
the spin structure function $g_{1}[35,36]$, the helicity-dependent structure functions $H_{1 / 2,3 / 2}[37]$, and the charged pion electroproduction in semi-inclusive scattering [38]. It was then postulated that duality is a universal feature of quark-hadron transition [39] that should present not only in electromagnetic interactions, but also in charged lepton scattering through the weak interaction, and maybe other processes as well. The resonance $A_{P V}$ measured during the PVDIS experiment can provide the first verification of the quark-hadron duality in the parity violating electron scattering process. 
2

\section{Experimental Setup}

The PVDIS experiment E08-011 [25] was performed from November to December of 2009 in Hall A at the Thomas Jefferson National Accelerator Facility (also referred to as Jefferson Lab, or JLab in short). During the experiment, the parity violating asymmetry of an electron beam scattering off an unpolarized liquid deuterium target was measured both in the deep inelastic scattering and in the resonance region. Most of the experiment's beam time was spent on the deep inelastic scattering kinematics, from which the quark weak axial coupling combination $2 C_{2 u}-C_{2 d}$ can be extracted. The resonance kinematics serve as auxiliary measurements for background study of the DIS's, while standing alone they also provide the first insight of the parity violating asymmetry in the whole resonance region. All these kinematics share the same experimental setup and instrumentation, which will be described in detail in this chapter. 


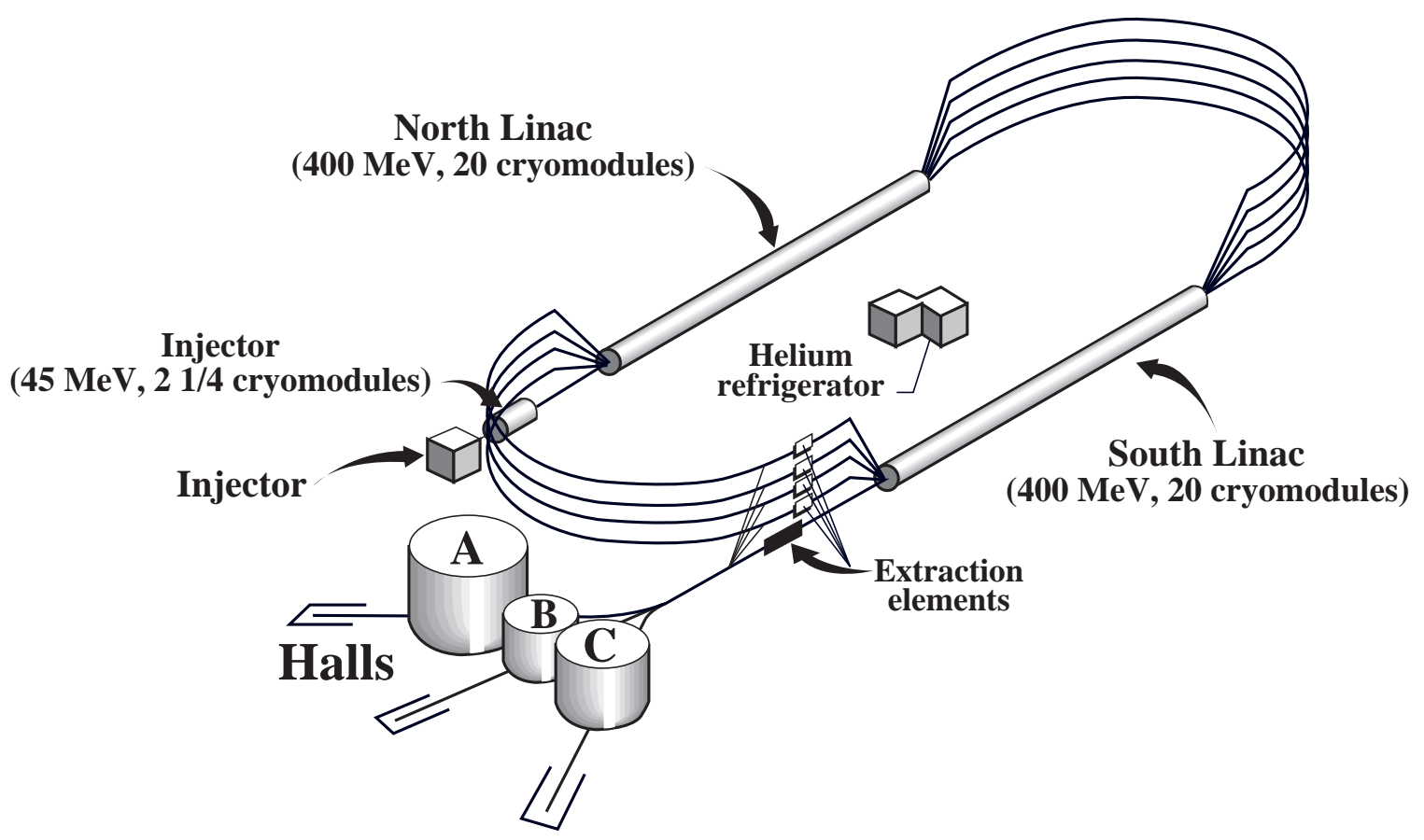

Figure 2.1: The Jefferson Lab accelerator.

\subsection{Accelerator}

The polarized electron beam at Jefferson Lab is generated by the Continuous Electron Beam Accelerator Facility (CEBAF), as pictured in Figure 2.1 Electrons can be accelerated up to $6 \mathrm{GeV}$ after been recirculated up to five times through two superconducting linacs (the North and the South Linac). Each linac is composed of 20 cryo -modules with a $5 \mathrm{MeV} / \mathrm{m}$ accelerating gradient by the initial design, which corresponds to a total energy gain of $400 \mathrm{MeV}$ for each linac. A full circulation through both linacs is usually referred to as one "pass". Later on research and development made it possible to push the energy gain higher when required by the experiment, and a total gain of about $1.2 \mathrm{GeV}$ each pass is achievable, resulting in a maximum beam energy of $6 \mathrm{GeV}$.

The polarized electron source is produced by illuminating a strained GaAs photocathode with circularly polarized laser light [40]. The absorption of laser light excites 
the electrons from the valence band $(\mathrm{J}=3 / 2)$ to the conduction band $(\mathrm{J}=1 / 2)$. The helicity state of the excited electron is determined by the polarization of the laser, with left (right) circular light producing positive (negative) helicity of the electron. By switching the polarization of the laser light using a Pockels Cell, one manages to flip the helicity state of the electron beam. A Wien Filter [41] is used to orient the polarization of the beam in the transverse direction. Electrons ejected from the photocathode are accelerated to approximately $45 \mathrm{MeV}$ first and then injected into the linac for acceleration.

The maximum beam current that CEBAF can operate with in the CW (continuous wave) mode is $200 \mu \mathrm{A}$, which is shared among the three experimental halls: Hall A, Hall B and Hall C. For independent control of the beams sent to different halls, three individual lasers, one for each hall, are used to illuminate one common photocathode at different time intervals. This produces three beam bunch trains, which are combined in an interleaving fashion in time and accelerated together. Each of the beam bunch train can be extracted at any desired pass and deflected into one of the halls using RF extracting units. Therefore, all three halls can run simultaneously with different beam energies, and different beam currents as well. During PVDIS, a $100 \mu \mathrm{A}$ beam with energy of $6 \mathrm{GeV}$ was delivered into Hall A during the DIS measurements and both $6 \mathrm{GeV}$ and $4.8 \mathrm{GeV}$ were used for the resonances.

\subsection{Experimental Hall A}

With a diameter of 53 meters, Hall $\mathrm{A}$ is the largest among the three experimental halls. A schematic of the Hall A layout during PVDIS is shown in Figure 2.2. The whole experimental setup can be categorized into three sets of instrumentation: the beamline, the target and a pair of high resolution spectrometers(HRS), which will be 


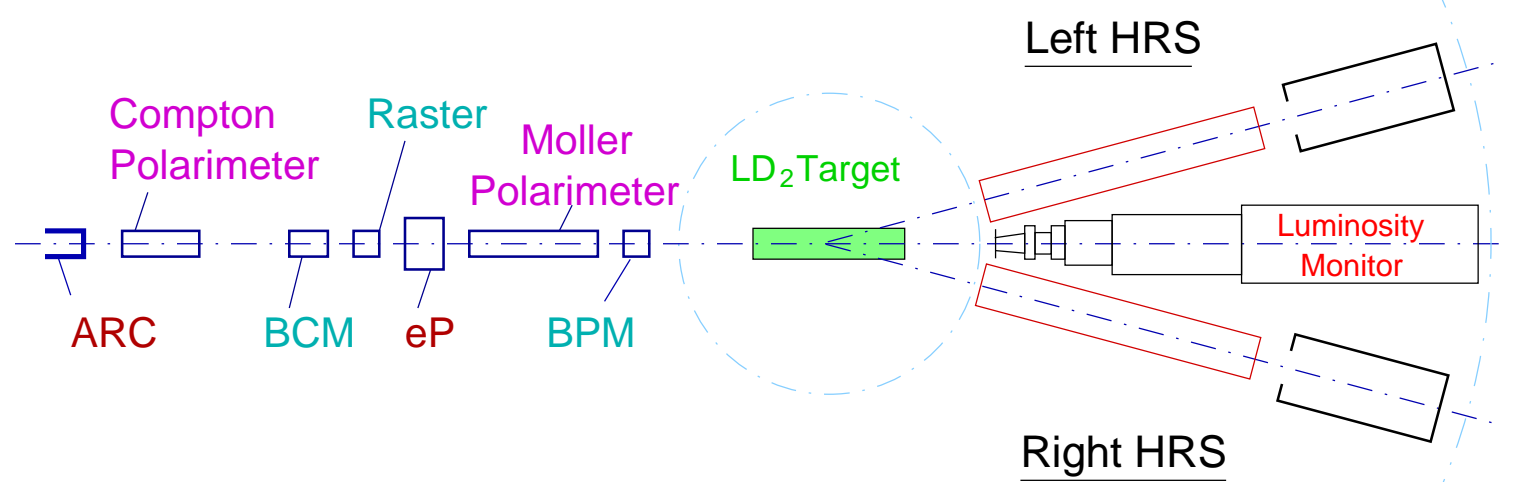

Figure 2.2: The layout of Hall A during PVDIS.

described in detail in the following sections.

\subsection{Beamline}

\subsubsection{Beam Helicity}

The helicity of the electron beam is determined by the helicity state of the circularly polarized laser, which is generated by passing a linearly polarized laser through a "Pockels Cell (PC)" before it hits the photocathode. A Pockels Cell is a crystal that acts as a quarter-wave retardation plate when a high voltage is applied on it. The polarity of the high voltage determines whether the Pockels Cell behaves like a positive or negative quarter-wave plate, which in turn determines the helicity of the circularly polarized laser and thus the helicity of the electron beam.

The polarity of the high voltage for the Pockels Cell is rapidly modulated by a helicity signal at a rate of $30 \mathrm{~Hz}$, which produces beam helicity windows of $33.3 \mathrm{~ms}$ in time. The helicity state sequence always shows up in a quartet pattern, as can be seen in Figure 2.3, either in a sequence of +--+ or of -++- , with the first helicity 


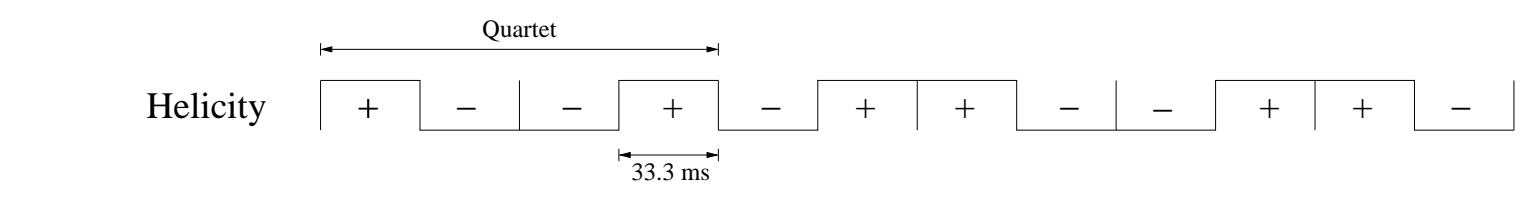

Delayed Helicity

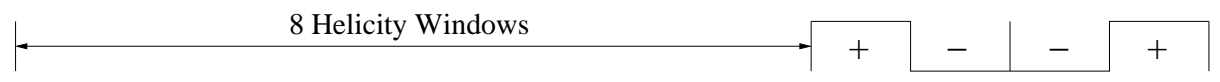

(a)

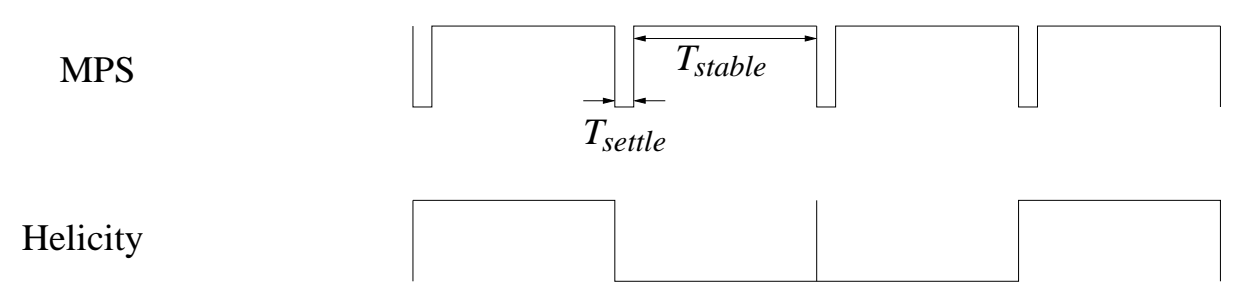

(b)

Figure 2.3: (a): Structure of the Helicity signal and the delayed Helicity signal; (b): Timing of the MPS signal with respect to the Helicity signal. The MPS signal is used to mute the transition period during change of Helicity states.

state of the quartet determined by a pseudo-random number generator. During the transition period between two adjacent helicity windows, the helicty state is unstable so any data taken during this period is not reliable. An MPS signal is specifically designed to deal with this issue. As shown in Figure 2.3, each MPS window is exactly the same length in time as a helicity window, and is composed of a $T_{\text {Settle }}$ part and a $T_{\text {Stable }}$ part. The $T_{\text {Settle }}$ is used to mute the DAQ for the helicity transition period and the leading edge of the $T_{\text {Stable }}$ resumes the DAQ for data taking.

All the helicity signals are generated by a Helicity Generator located at the Machine Control Center (MCC) and isolated from all other equipments. The Helicity Generator runs on its own battery and uses fiber optical cables to transmit the output signals. This total isolation from the $60 \mathrm{~Hz} \mathrm{AC}$ line suppresses any false asymmetries generated from helicity correlated electronic pickups. To further minimize any potential systematic effects, the helicity signals are delayed by eight windows (Figure 2.3) before being sent to the experimental halls during PVDIS. 
In addition to the fast flip of helicity using the Pockels Cell, a slower helicity reversal technique is available by using an Insertable Half-Wave Plate (IHWP), located upstream of the Pockels Cell. The IHWP can be inserted into and retracted from the laser path whenever necessary, and the helicity state of the electron beam when the IHWP is inserted is opposite to the case when it is not. This provides a powerful way of checking for false asymmetries because the insertion of IHWP should only flip the sign of the measure physical asymmetry without introducing any extra effects. During PVDIS, the IHWP was inserted into or retracted from the laser path about once every day of smoothing running, allowing for continuous monitoring of slow helicity-correlated systematics.

\subsubsection{Beam Energy Measurement}
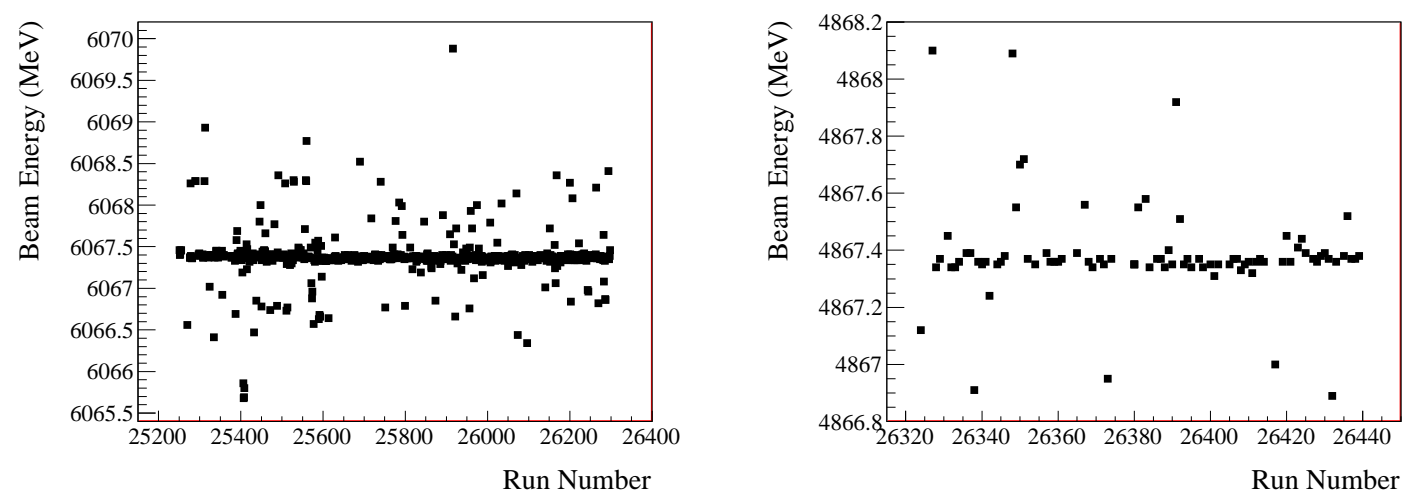

Figure 2.4: Beam energy versus run number. The left and right plots represent two different beam energy settings during PVDIS.

There are three methods for absolute beam energy measurement:

- Arc Measurement: this measurement takes place at the arc section of the beamline and is based on the principle that an electron moves in a circular pattern in a magnetic field. By simultaneously measuring the field integral (the so-called $\int \vec{B} \cdot \overrightarrow{d l}$ value in T.m) of the eight dipoles and the net bend angle $\theta$ at the 
arc section, one can determine the momentum of the electron according to the relation

$$
p=c \frac{\int B_{\perp} \cdot d l}{\theta}
$$

where $c=0.299792 \mathrm{GeV} / \mathrm{Tm}$ is the speed of light, $B_{\perp}$ is the magnetic field component perpendicular to the electron motion and $l$ represents the total path length of the electron through the magnetic field. The precision of this measurement is $\delta E / E \approx 2 \times 10^{-4}$.

- eP Measurement: The eP measurement can be performed using a stand-alone device located upstream of the target along the beamline [42]. It is based on the elastic e-P scattering process. The precision of this measurement is also $\delta E / E \approx 2 \times 10^{-4}$.

- Tiefenbach Measurement: This measurement uses the current values of Hall A $\operatorname{arc} \int \vec{B} \cdot \overrightarrow{d l}$ value and Hall A arc beam position monitors (BPMs) to calculate the beam energy. This number is continuously recorded in the data stream and is calibrated against the arc energy of a reference magnet regularly. The precision of this measurement is $\delta E / E \approx 1 \times 10^{-3}$.

Both Arc measurement and eP measurement are invasive as the production data taking has to be stopped to perform the measurements. Therefore, the Tiefenbach method is chosen for continuous monitoring of the beam energy and its fluctuations. The beam energy recorded throughout PVDIS is shown in Figure 2.4. 


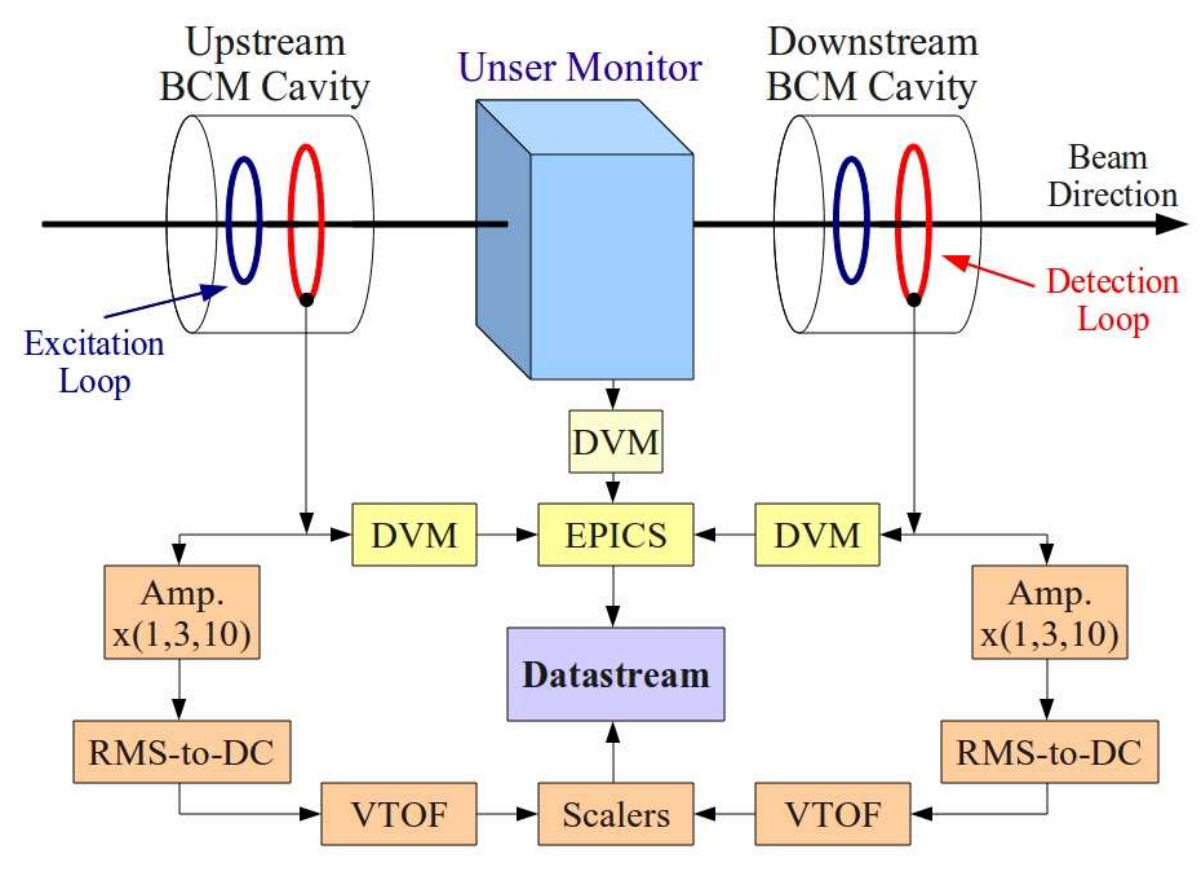

Figure 2.5: Schematic of beam current monitors.

\subsubsection{Beam Current}

\subsubsection{Beam Current Monitors}

The beam current in Hall A is measured by beam current monitors (BCM) [43] located 25 meters upstream of the target. Figure 2.5 shows a sketch of the measuring system, which consists of an Unser monitor, two RF cavities (the up- and down-stream BCM cavities) and the accessory data acquisition system. The Unser monitor is a parametric current transformer that provides an absolute measure of the beam current. However, it is not desirable to use the Unser monitor for continuous measurement of beam current because its output signal drifts significantly over a time scale of a few minutes. Rather, the Unser monitor is used for calibration of the other two RF cavities, which are placed on either side of the Unser and used for continuous monitoring of beam current. The RF cavity monitors are stainless steel cylindrical waveguides with high quality factor $(\mathrm{Q} \approx 3000)$ and tuned to the frequency of the beam. The 
electron beam passing through the cavity excites its resonance mode and produces a signal proportional to the beam current.

The output signal from each of the cavities is splitted into two paths for different measuring and recording processes. One part of the signal is fed to a high-precision AC voltmeter, which provides a digital output every second corresponding to the RMS value of the input. This output is logged as a slow control (EPICS, see Section 2.6 variable every 1 to 2 seconds. The other part of the signal is amplified with different gains $(\mathrm{x} 1, \mathrm{x} 3, \mathrm{x} 10)$ and then sent to RMS-to-DC converters which produce analog DC voltage levels. Amplification is necessary here because the RMS-to-DC output is only linear for currents from about $5 \mu \mathrm{A}$ to $200 \mu \mathrm{A}$. The $\mathrm{x} 3$ and $\mathrm{x} 10$ amplifications can extend the linear region to lower currents at the expense of saturation at high currents. The outputs from the RMS-to-DC converters are sent to Voltage-to-Frequency converters, producing digital pulses of frequencies proportional to the voltage levels, which are then fed to VME scalers for read out and are recorded in the data stream. The scalers run in integrating mode, with the counts representing the accumulated beam charge.

The data recording scheme for beam current measurements described above is the standard method implemented in the so called HRS DAQ. During PVDIS, as in other parity experiments in Hall A (HAPPEX I III and PREX), another separate DAQ system (See Section 2.6) named Parity DAQ was also running in parallel with the HRS DAQ. All BCM signals, including the Unser signal and cavity signals with different amplifications, are copied and sent to the ADCs of the HAPPEX DAQ for integration. During data analysis of PVDIS, the ADC measurement of the upstream cavity signal with gain $\mathrm{x} 1$ was chosen for beam intensity normalization of the measured asymmetry. 


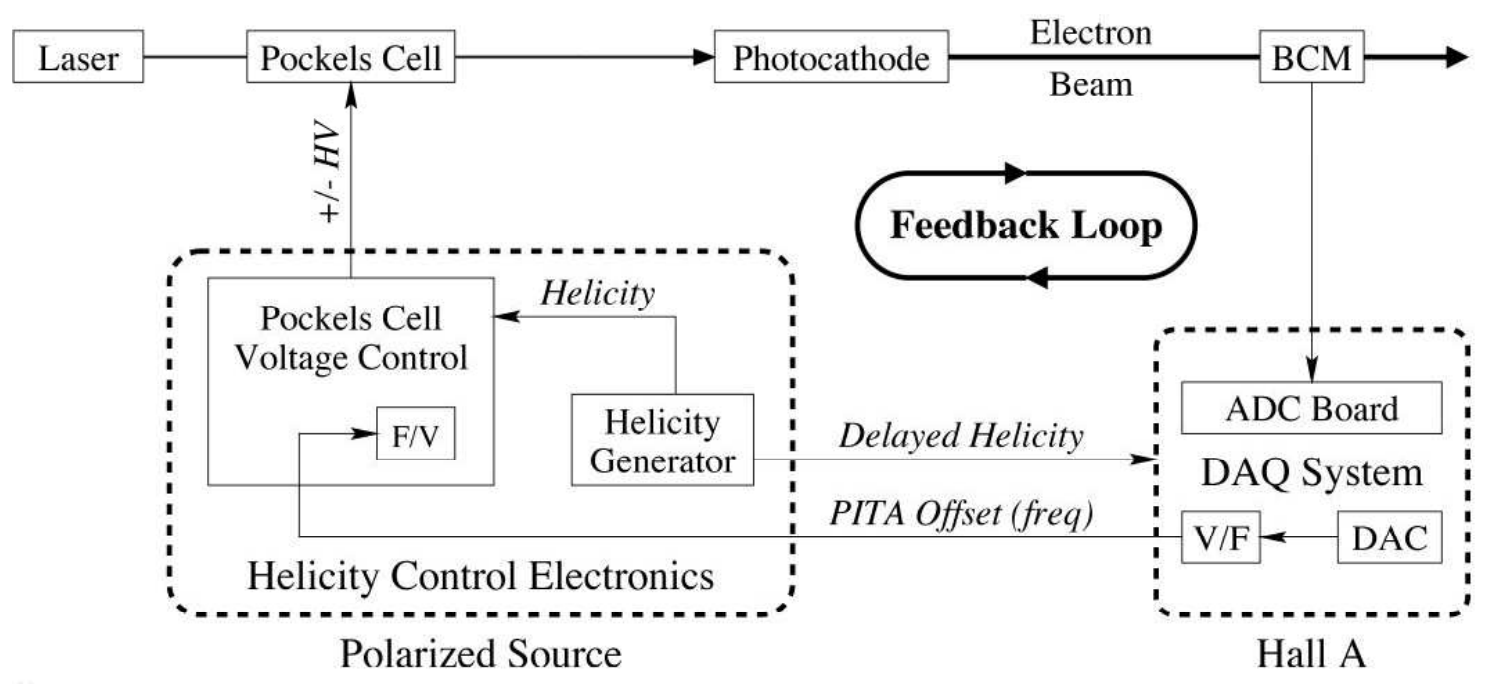

Figure 2.6: Beam charge asymmetry feedback system.

\subsubsection{Charge Asymmetry Feedback}

Beam charge asymmetry arises from the difference in beam intensity between different helicity states and can be defined as:

$$
A_{I}=\frac{I^{+}-I^{-}}{I^{+}+I^{-}}
$$

This beam intensity difference originates from the intensity difference of the circularly polarized laser hitting the photocathode and can be minimized by using the Polarization-Induced Transport Asymmetry (PITA) effect [44]. It is achieved by applying a small voltage offset asymmetric for the two helicity states, called the PITA voltage, onto the Pockels Cell in order to cancel the laser intensity difference induced by the circular polarization process. The PITA voltage to be applied depends on the value of the charge asymmetry. The coefficient relating the two, called the PITA slope, can be determined from diagnostic beam study.

A real time feedback system is implemented to continuously minimize the charge asymmetry, as shown in Figure 2.6. During the experiment running, data from the 


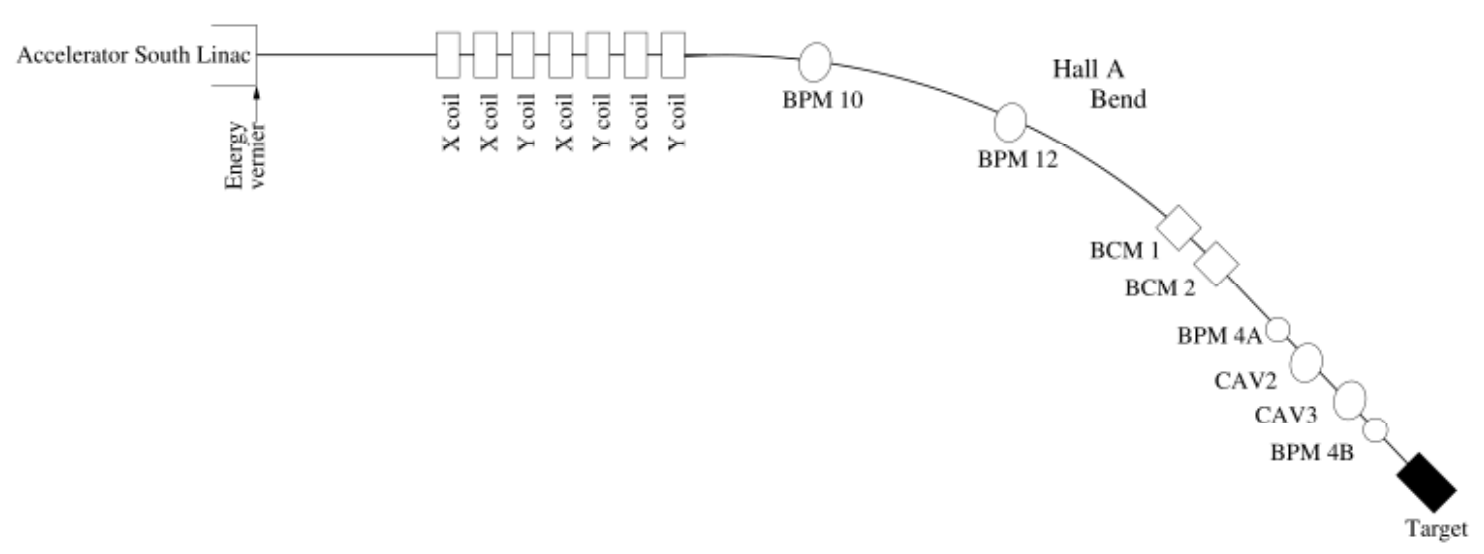

Figure 2.7: Layout of the beam position monitors.

BCMs was analyzed concurrently and a charge asymmetry was produced about every two minutes, based only on the data taken in the last two minutes. With the predetermined PITA slope, the PITA offset voltage can be calculated and sent to the control electronics of the Pockels Cell. With this feedback system running, the charge asymmetry can be minimized to sub-ppm level after about one hour.

\subsubsection{Beam Position}

\subsubsection{Beam Position Monitors}

Helicity-correlated differences in the beam position and energy are important sources of false asymmetry. Therefore, it is essential to monitor these properties accurately and correct for any systematics related to them. At Jefferson Lab, the beam position is measured by stripline monitors [45] consisting of four wire antennae oriented parallel to the beam direction and placed symmetrically around the beam pipe. Each wire provides a signal that is related to both the beam position and intensity. During PVDIS, two sets of such Beam Position Monitors (BPMs) were used, named BPM4A and BPM4B, which were located $7.524 \mathrm{~m}$ and $1.286 \mathrm{~m}$ upstream of the target respectively. The exact beam position and incident angle on the target were extrapolated 
from the measurements provided by these two BPMs. Besides, an additional BPM named BPM12x, which was located at a highly dispersion point of the arc section of the beamline, was used to monitor any helicity-correlated fluctuations in the beam energy.

\subsubsection{Beam Modulation}
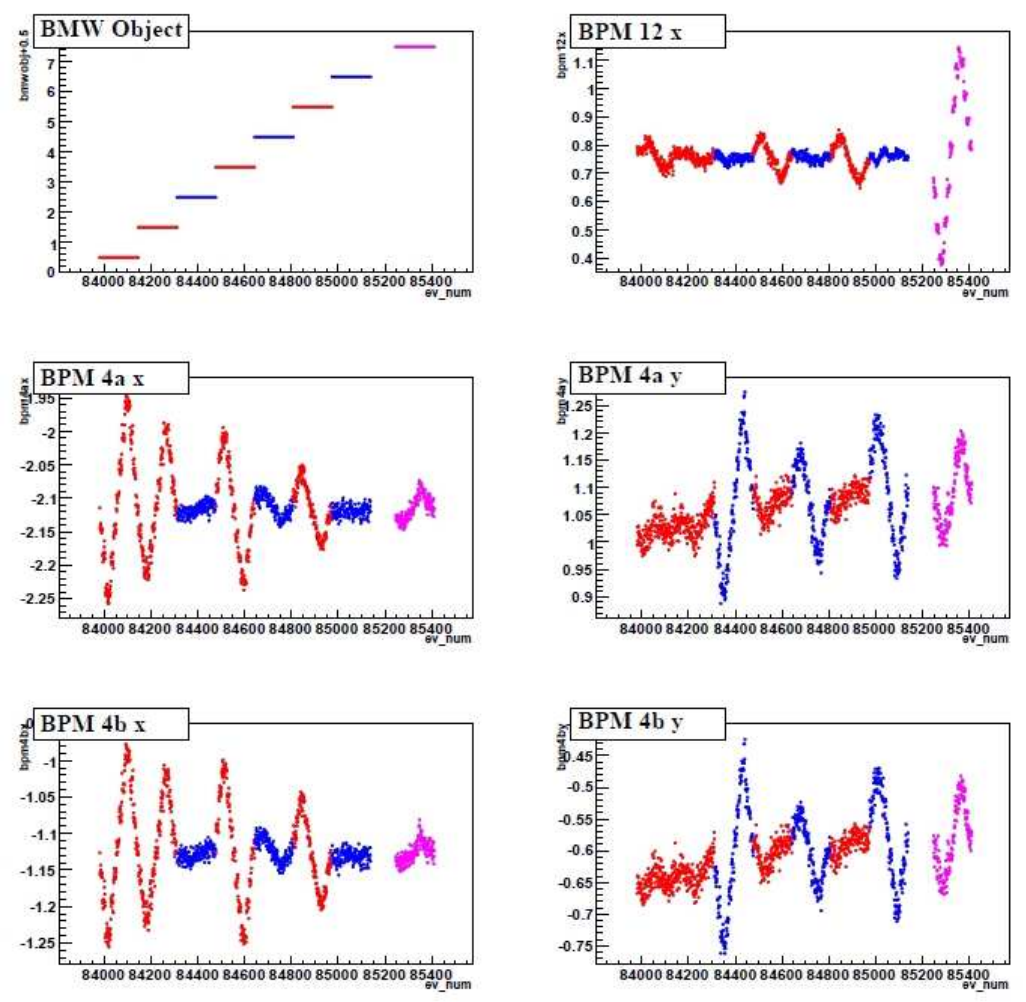

Figure 2.8: One example cycle of the beam modulation ("dithering") procedure.

In order to correct for any false asymmetry caused by the beam movement and energy fluctuation, in addition to measuring the beam position and energy accurately, we also need to know the sensitivity of the physical asymmetry to various beam parameters. This sensitivity can be studied using a beam modulation technique (also referred to as "dithering") [46], which uses a set of seven modulation coils to move 
the beam around horizontally and vertically, and an energy vernier to modulate the beam energy. The coils are located about $7 \mathrm{~m}$ upstream of the arc section (Figure 2.7) while the vernier sits at the end of the accelerator's south linac.

During the experiment, the beam modulation process was turned on about every 10 minutes and each full modulation cycle lasted for about 1 minute. During a typical cycle, each of seven coils was modulated successively, resulting in independent movements of the beam in different directions, followed by the energy modulation at the end. Figure 2.8 shows how the BPM signals vary accordingly during one modulation cycle. Detailed analysis of the beam modulation data is presented in Section 3.4.2.1, as well as the necessary corrections made to the raw asymmetry.

\subsubsection{Raster}

The high intensity beam produced by the accelerator has an intrinsic beam spot size of $100 \sim 200 \mu \mathrm{m}$ and can cause local over-heating of the target, which might create target density fluctuations that contribute nonstatistical noise to the asymmetry measured. To avoid this, a fast rastering system is used to sweep the beam over a rectangular area with side length up to several millimeters. This is done by two sets of horizontal and vertical air-core dipoles located 23 meters upstream of the target. During PVDIS, a raster size of $4 \times 4 \mathrm{~mm}^{2}$ was used (see Figure 2.9).

\subsubsection{Beam Polarization}

Precise knowledge of the beam polarization is crucial to parity experiments because it affects normalization of the raw asymmetry. At Jefferson Lab, there are three different polarimeters to measure the beam polarization: the Mott polarimeter, the Møller polarimeter and the Compton polarimeter. Each of these three polarimeters provides an independent measurement of the beam polarization and can be cross- 


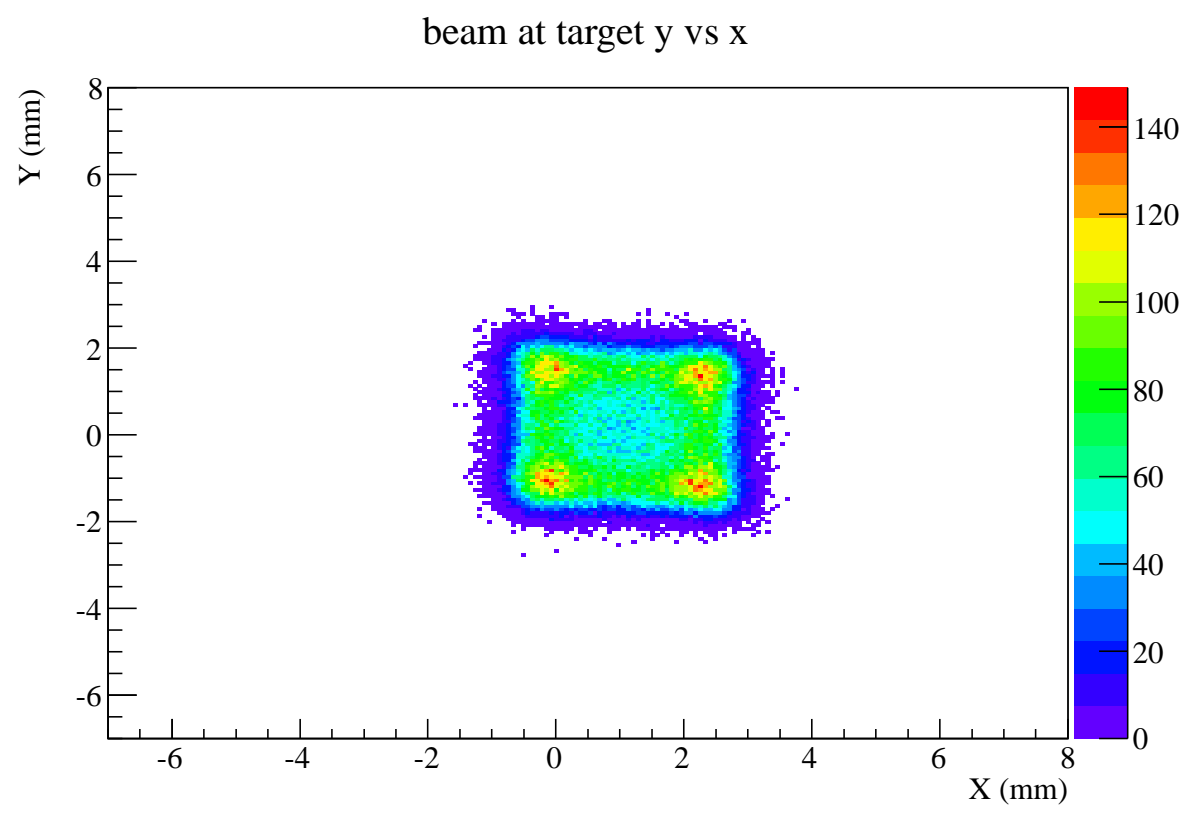

Figure 2.9: Beam raster during PVDIS.

checked with each other. The Mott and Møller measurements are invasive while the Compton polarimeter provides continuous non-invasive monitoring of the beam polarization. During PVDIS, both the Møller and the Compton polarimeter were used.

\subsubsection{Mott Polarimeter}

A Mott polarimeter $[47,48,49]$ is located near the injector to the first linac where the electrons have reached $5 \mathrm{MeV}$ in energy. Mott polarimeter is based on the scattering of polarized electrons from unpolarized high- $Z$ nuclei. The spin-orbit interaction of the electron's spin with the magnetic field it sees due to its motion relative to the nucleus causes a differential cross section

$$
\sigma(\theta)=I(\theta)\left[1+S(\theta) \vec{P}_{e} \cdot \widehat{n}\right],
$$


where $\theta$ is the Mott scattering angle, $S(\theta)$ is the theoretical Sherman function, $\vec{P}_{e}$ is the electron beam spin vector, and $I(\theta)$ is the spin-averaged scattering cross section

$$
I(\theta)=\frac{Z^{2} e^{4}}{4 m^{2} \beta^{4} c^{4} \sin ^{4}(\theta / 2)}\left[1-\beta^{2} \sin ^{2}(\theta / 2)\right]\left(1-\beta^{2}\right)
$$

The unit vector $\widehat{n}$ is normal to the scattering plane, defined by $\widehat{n}=\left(\vec{k} \times \vec{k}^{\prime}\right) /\left|\vec{k} \times \vec{k}^{\prime}\right|$ where $\vec{k}$ and $\vec{k}^{\prime}$ are the electron's momentum before and after scattering, respectively. Thus $\sigma(\theta)$ depends on the component of the electron beam polarization perpendicular to the Mott scattering plane, $P_{e}$. Defining an asymmetry

$$
A(\theta)=\frac{N_{L}-N_{R}}{N_{L}+N_{R}}
$$

where $N_{L}$ and $N_{R}$ are the number of electrons scattered to the left and right, respectively, one has

$$
A(\theta)=P_{e} S(\theta)
$$

Knowledge of the Sherman function $S(\theta)$ therefore allows $P_{e}$ to be extracted from the measured asymmetry with a precision of $3 \%[16,50]$. Since Mott polarimeter measures the polarization at the injector, results from Mott polarimeter were not used directly for asymmetry corrections. However, the Mott polarimeter was used for setting up and verifying the transversely-polarized beam for the beam normal asymmetry $A_{n}$ measurement.

\subsubsection{Møller Polarimeter}

The Møller polarimeter [43], located on the Hall A beamline about 17.5 meters upstream of the target, measures the beam polarization based on the Møller scattering between the polarized electron beam and polarized atomic electrons in a magnetized 

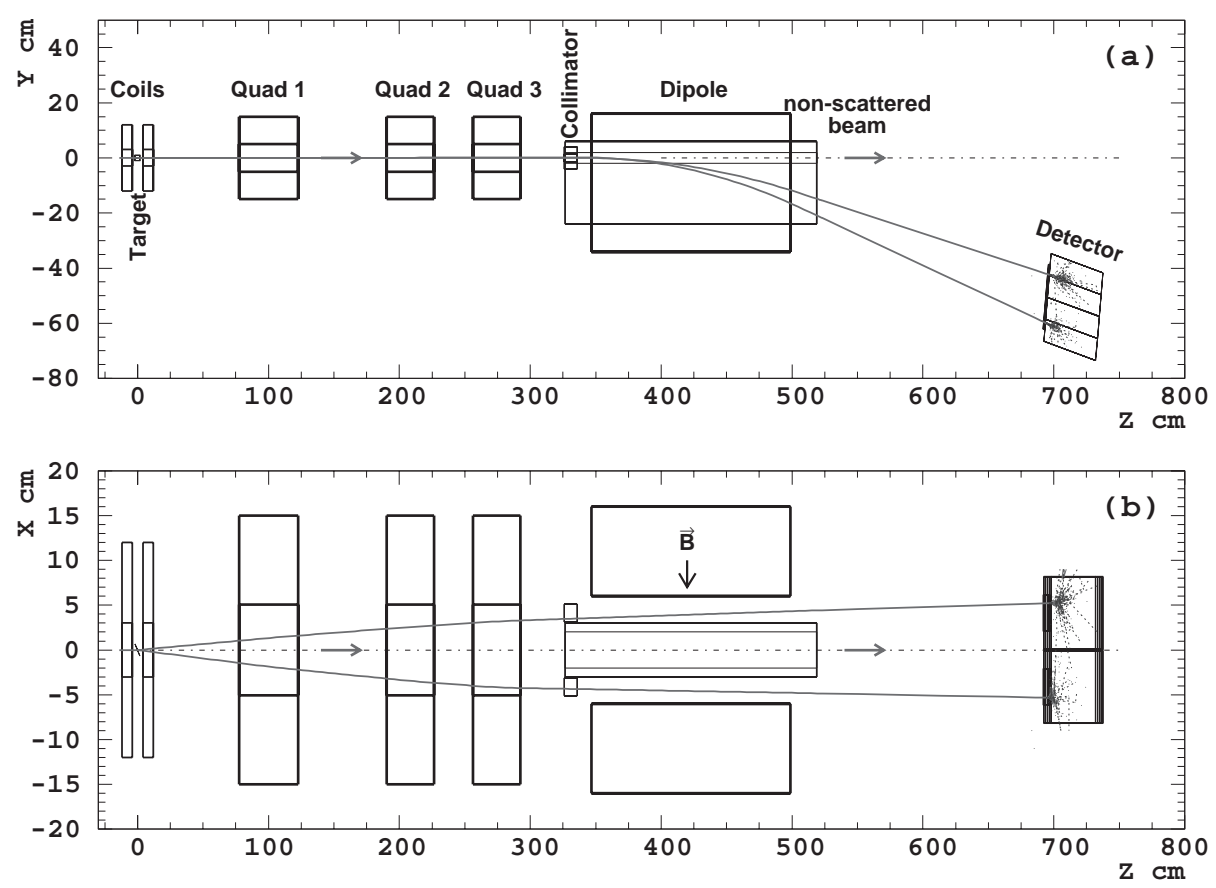

Figure 2.10: Layout of the Møller polarimeter

foil. The spin-dependent cross section of the Møller scattering has a component proportional to both the beam polarization $P^{\text {beam }}$ and the target polarization $P^{\text {target: }}$

$$
\sigma \propto\left(1+\sum_{i=X, Y, Z} A_{i i} P_{i}^{\text {beam }} P_{i}^{\text {target }}\right),
$$

where $i=X, Y, Z$ are orthogonal projections of the polarization, and the analyzing power $A_{i i}$ depends on the scattering angle in the center of mass frame $\theta_{\text {CMS }}$. Assuming that the scattering happens in the ZX plane with the Z-axis defined as the beam direction, the three components of the analyzing power can be written as:

$$
\begin{aligned}
& A_{Z Z}=-\frac{\sin ^{2} \theta_{\mathrm{CMS}}\left(7+\cos ^{2} \theta_{\mathrm{CMS}}\right)}{\left(3+\cos ^{2} \theta_{\mathrm{CMS}}\right)^{2}} \\
& A_{X X}=-\frac{\sin ^{4} \theta_{\mathrm{CMS}}}{\left(3+\cos ^{2} \theta_{\mathrm{CMS}}\right)^{2}} \\
& A_{Y Y}=-A_{X X}
\end{aligned}
$$


The longitudinal beam polarization can be extracted from the measured Møller asymmetry using the relation:

$$
P_{Z}^{\text {beam }}=\frac{A_{\text {Møller }}}{P_{Z}^{\text {target }}\left\langle A_{Z Z}\right\rangle}
$$

where $A_{\mathrm{M} \varnothing l \text { ler }}$ is the experimentally measured asymmetry and $P_{Z}^{\text {target }}$ is the target polarization along the beamline. The quantity $\left\langle A_{Z Z}\right\rangle$ is the mean analyzing power, which is calculated using a Monte-Carlo to average $A_{Z Z}$ over the whole Møller spectromemter acceptance.

The target of the Møller polarimeter is a ferromagnetic foil magnetized in a magnetic field of $24 \mathrm{mT}$ along its main symmetry axis. The target polarization is measured by a dedicated offline procedure and is usually around $8 \%$. The orientation of the foil plane can be at various angles with respect to the beam direction, meaning the target polarization may also have a transverse component that can couple with the beam's transverse polarization. In order to suppress contributions from the transverse polarization, the Møller asymmetry is measured at two target angles $\left( \pm 20^{\circ}\right)$ and the average is taken.

Figure 2.10 shows the layout of the Møller polarimeter setup. The scattered electrons are detected by a magnetic spectrometer consisting of a sequence of three quadrupoles and a dipole. The detector package consists of scintillators and lead-glass calorimeter modules, which are split into two arms in order to detect the two scattered electrons in coincidence. The spectrometer allows of a center-of-mass scattering angle in the range of $75^{\circ} \leqslant \theta_{\mathrm{CMS}} \leqslant 105^{\circ}$, with a central $A_{Z Z}$ (the maximum value at $\left.\theta_{\mathrm{CMS}}=90^{\circ}\right)$ of about $7 / 9$.

The Møller measurement is invasive so it was only carried out once every several days, or when significant kinematic changes took place. It usually takes about an hour to acquire a $0.2 \%$ statistical accuracy, and the total systematic uncertainty is 
typically $2 \%$ relative, dominated by the Møller target polarization. Beam polarization results from Møller measurements during PVDIS can be found in Section 3.6.2.

\subsubsection{Compton}

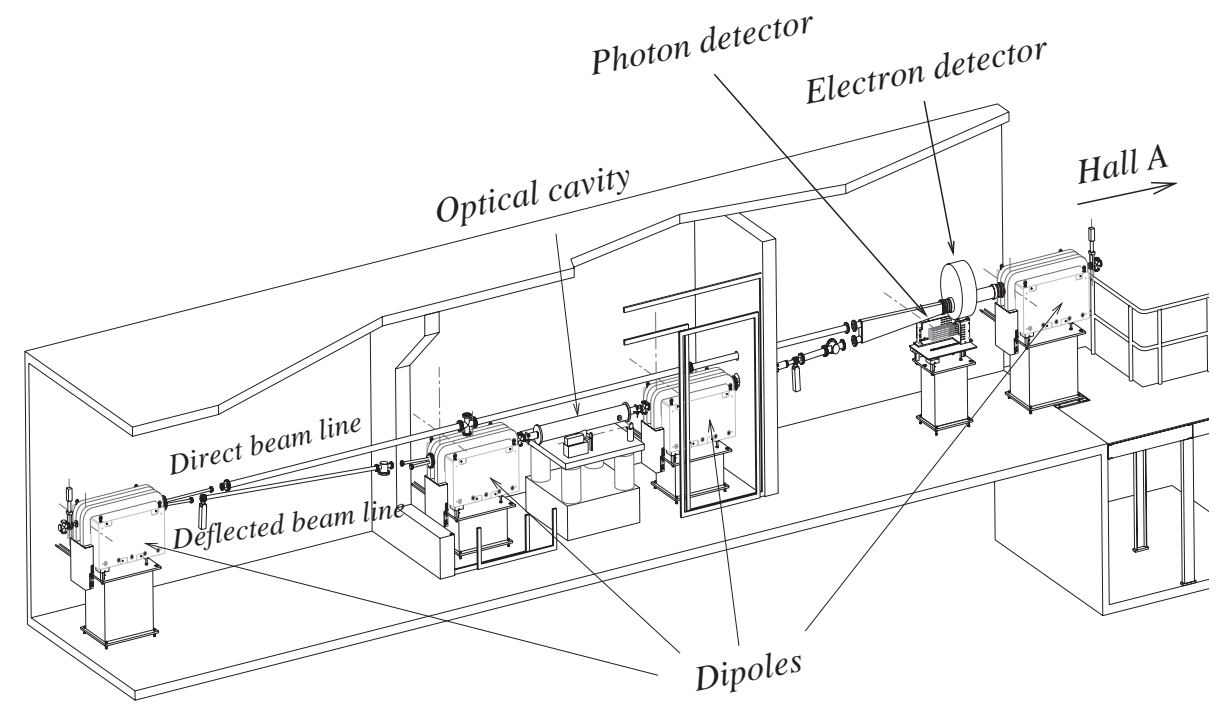

Figure 2.11: Layout of the Compton polarimeter

The beam polarization can also be measured by the Compton polarimeter [ $[51,52$, 53, 54], which utilizes the Compton scattering of polarized electrons with a circularly polarized photon beam. Unlike the Møller polarimeter, the Compton polarimeter provides non-invasive measurements of the beam polarization because the Compton scattering cross-section is very low (only 1 out of about $10^{9}$ electrons gets scattered).It measures the asymmetry between the energy-weighted, integrated Compton signal $S$ at different helicity states:

$$
A_{\text {exp }}=\frac{S^{+}-S^{-}}{S^{+}+S^{-}}=P_{b} P_{\gamma} A_{t h}
$$


where $A_{\text {exp }}$ is the experimentally measured Compton scattering asymmetry, $P_{\gamma}$ is the polarization of the photon beam which can be measured directly, and $A_{t h}$ is the theoretically calculated Compton scattering asymmetry assuming 100\% of photon and electron polarizations. Therefore, the beam polarization $P_{b}$ can be extracted according to Equation 2.3.10.

The Compton polarimeter in Hall A is located at the entrance of the hall, after the beam comes out of the arc. Figure 2.11 shows a schematic representation of the polarimeter's layout. The electron beam entering from the left is directed into the Compton chicane by a pair of dipoles, where a high power polarized laser beam is prepared using a Fabry-Perot cavity [55]. At the center of the cavity, the electron beam undergo Compton scattering with the photon beam. The crossing angle between the two beams is $23 \mathrm{mrad}$. In order to control for systematic effects, the laser polarization is periodically flipped between left- and right-circular states. In between flips of the laser polarization, the laser is turned off for background measurements. After Compton scattering, the major portion of the electron beam remains untouched and is guided by another pair of dipoles to return to the normal beam line. Scattered electrons are detected by an electron detector made of silicon microstrips and scattered photons are detected by a photo detector consisting a GSO crystal calorimeter. Unfortunately, the electron detector was not functional during PVDIS, so the Compton measurements of beam polarization were performed with the photon detector alone.

The photon detector was equipped with an integrating Flash ADC (FADC) data acquisition (DAQ) system. The signal from the photon detector was sampled by the FADC at $200 \mathrm{MHz}$ and integrated into a digital summing accumulator for every helicity window, which was then read out and recorded in the data stream. The energyweighted Compton asymmetry can be calculated according to Equation 2.3.10 using the accumulator values. Compared to a counting method, the integrating method 
has obvious advantages in that it is not sensitive to threshold, pileup and deadtime effects that are inherent in a counting method. Also, the integrating method eliminates the need for precise calibration of the Compton spectrum, which allows a precise stand-alone measurement using only the photon detector.

More description of the Compton photon detector and its accompanying DAQ system can be found in $[53,54]$. Analysis of the Compton measurements during PVDIS is presented in Section 3.6.

\subsection{Target}

\subsubsection{Cryogenic Target System}

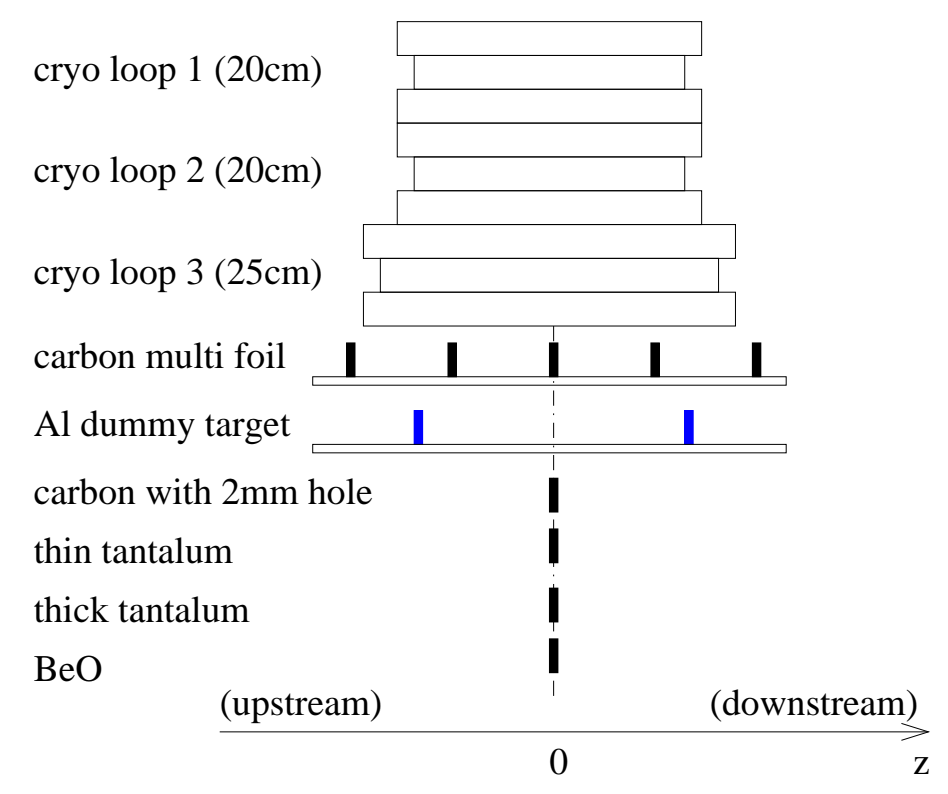

Figure 2.12: Schematic diagram of the target ladder arrangement used during the experiment. The electron beam is along the horizontal direction (the $z$-axis) and is incident from the left on the target. The carbon multi-foils were located at $z=$ $(-15,-7,0,7,15) \mathrm{cm}$ and the Al dummy foils were located at $z=(-10,10) \mathrm{cm}$. All other solid targets were located at $z=0 \mathrm{~cm}$ and were about 1 inch apart in the vertical direction. 


\begin{tabular}{|c|c|c|c|}
\hline Target & Position and material & Purity & Thickness \\
\hline cryo-loop 1 & $\begin{array}{c}\text { Entrance window,-10 } \mathrm{cm}, \mathrm{Al}^{a} \\
\text { Exit window, }+10 \mathrm{~cm}, \mathrm{Al} \\
\text { Wall, beam left upstream, } \mathrm{Al} \\
\text { Wall, beam left middle, } \mathrm{Al} \\
\text { Wall, beam left downstream, } \mathrm{Al} \\
\text { Wall, beam right upstream, } \mathrm{Al} \\
\text { Wall, beam right middle, } \mathrm{Al} \\
\text { Wall, beam right downstream, } \mathrm{Al}\end{array}$ & & $\begin{array}{l}0.126 \pm 0.011 \pm 0.003 \mathrm{~mm}^{c} \\
0.100 \pm 0.008 \pm 0.003 \mathrm{~mm} \\
0.313 \pm 0.008 \pm 0.003 \mathrm{~mm} \\
0.317 \pm 0.002 \pm 0.003 \mathrm{~mm} \\
0.323 \pm 0.003 \pm 0.003 \mathrm{~mm} \\
0.340 \pm 0.002 \pm 0.003 \mathrm{~mm} \\
0.336 \pm 0.007 \pm 0.003 \mathrm{~mm} \\
0.313 \pm 0.008 \pm 0.003 \mathrm{~mm}\end{array}$ \\
\hline $\begin{array}{l}\text { Carbon multi-foil } \\
\text { Al Dummy }\end{array}$ & $\begin{array}{c}(-15,-7,0,7,15) \mathrm{cm}, \mathrm{C} \\
-10 \mathrm{~cm}, \mathrm{Al}^{a} \\
+10 \mathrm{~cm}, \mathrm{Al}\end{array}$ & $99.5 \%$ & $\begin{array}{c}0.042 \pm 0.001 \mathrm{~g} / \mathrm{cm}^{2} \text { (all foils) } \\
0.359 \pm 0.0003 \mathrm{~g} / \mathrm{cm}^{2} \\
0.367 \pm 0.0003 \mathrm{~g} / \mathrm{cm}^{2}\end{array}$ \\
\hline Carbon hole ${ }^{b}$ & $0 \mathrm{~cm}, \mathrm{C}$ & $99.95 \%$ & $0.08388 \pm 0.00012 \mathrm{~g} / \mathrm{cm}^{2}$ \\
\hline Tantalum Thin & $0 \mathrm{~cm}, \mathrm{Ta}$ & $99.9 \%$ & $0.021487 \pm 0.000078 \mathrm{~g} / \mathrm{cm}^{2}$ \\
\hline Tantalum Thick & $0 \mathrm{~cm}, \mathrm{Ta}$ & $99.9 \%$ & $0.12237 \pm 0.000341 \mathrm{~g} / \mathrm{cm}^{2}$ \\
\hline $\mathrm{BeO}$ & $0 \mathrm{~cm}, \mathrm{BeO}$ & $99.0 \%$ & $0.149 \pm 0.001 \mathrm{~g} / \mathrm{cm}^{2}$ \\
\hline
\end{tabular}

${ }^{a}$ All aluminum used for the cryo-target and the Al Dummy are made from Al 7075 T-6 plates.

${ }^{b}$ Both Al Dummy and Carbon Hole targets had a 2-mm hole to locate the target with the beam and to determine the target motion.

${ }^{c}$ The first error bar comes from the standard deviation of multiple measurements at different positions on the target, and the second error is from calibration of the instrument.

Table 2.1: Position, material, and thickness for the target system used in this experiment. The position is defined along the beam direction with respect to the hall center. 
The Hall A cryogenic target system [43] was used for this experiment. We used a 20-cm long deuterium target cell for the main production data-taking. Solid targets were used for evaluating backgrounds, studying the spectrometer optics, and checking beam centering. The target cell and a solid target ladder sit in an evacuated cylindrical scattering chamber of $104 \mathrm{~cm}$ diameter, centered on the pivot for the spectrometers. Also located inside the scattering chamber were subsystems for cooling, temperature and pressure monitoring, target motion, gas-handling and controls. The scattering chamber was maintained under a $10^{-6}$ Torr vacuum. The exit windows on the scattering chamber allowed scattered particles to reach the spectrometers. These windows were made of $0.406-\mathrm{mm}$ thick $\mathrm{Al}$ foil. Figure 2.12 shows a schematic diagram of the target ladder arrangement used during this experiment. Among the three cryogenic loops, only loop 1 was used.

The liquid deuterium loop (loop 1) was operated at a temperature of $22 \mathrm{~K}$ and a pressure of 25 psia, leading to a density of about $0.1676 \mathrm{~g} / \mathrm{cm}^{3}$. The walls of the loop 1 cell were made of aluminum. The thickness of loop 1 walls and up- and down-stream windows and those of solid targets are summarized in Table 2.1.

\subsubsection{Target Boiling}

As described in Section 2.3.4.3, the beam was rastered to a $4 \times 4 \mathrm{~mm}^{2}$ square spot at the target to spread the heat load on the target. Heat deposit in the liquid deuterium causes additional noise to the measurement. This effect, often referred to as the "local boiling" effect (although it is not an actual phase change of the liquid deuterium), would manifest itself as an increase in the standard deviation of the measured pairwise asymmetry $A^{\text {raw }}$ above that expected from the counting statistics. Studies of the local boiling effect was performed by measuring the fluctuation (the standard deviation) of pair-wise asymmetries. Figure 2.13 shows the measured standard deviation 


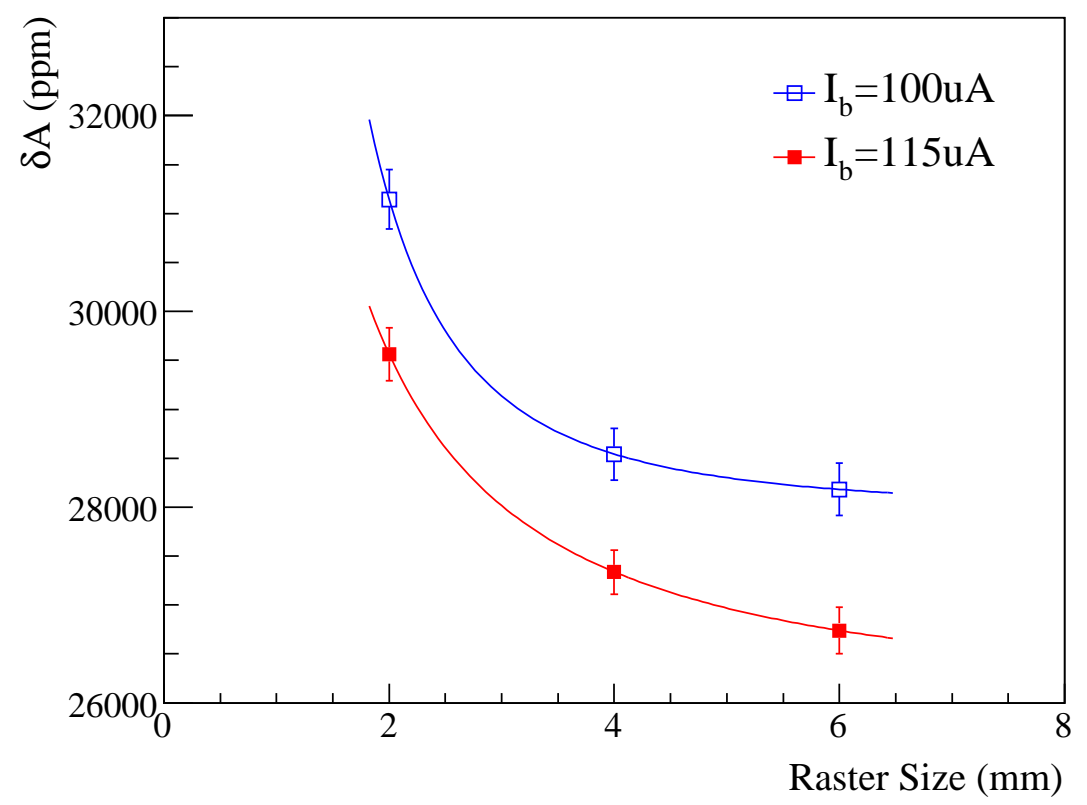

Figure 2.13: Measured standard deviation of the pair-wise asymmetries at kinematics DIS\# 2, for various raster sizes and two beam currents 100 and $115 \mu \mathrm{A}$. The curves show results of fit $\delta A=p_{0} x^{p_{1}}+p_{2}$ where $x$ is the raster size (side size of square) in $\mathrm{mm}$. The fit results for $100 \mu \mathrm{A}$ are $p_{0}=(1.77 \pm 1.94) \times 10^{4}, p_{1}=-2.48 \pm 1.85$, $p_{2}=27973.0 \pm 681.7$; and for $115 \mu \mathrm{A}$ are $p_{0}=(9.40 \pm 3.78) \times 10^{3}, p_{1}=-1.37 \pm 1.09$, $p_{2}=25941.0 \pm 1433.4$. At a raster size of $4 \times 4 \mathrm{~mm}^{2}(x=4)$, the boiling noise is at the level of $569 \mathrm{ppm}$ (1407 ppm) for $100(115) \mu \mathrm{A}$, and is negligible compared to the value from purely statistical fluctuations (the $p_{0}$ ). 

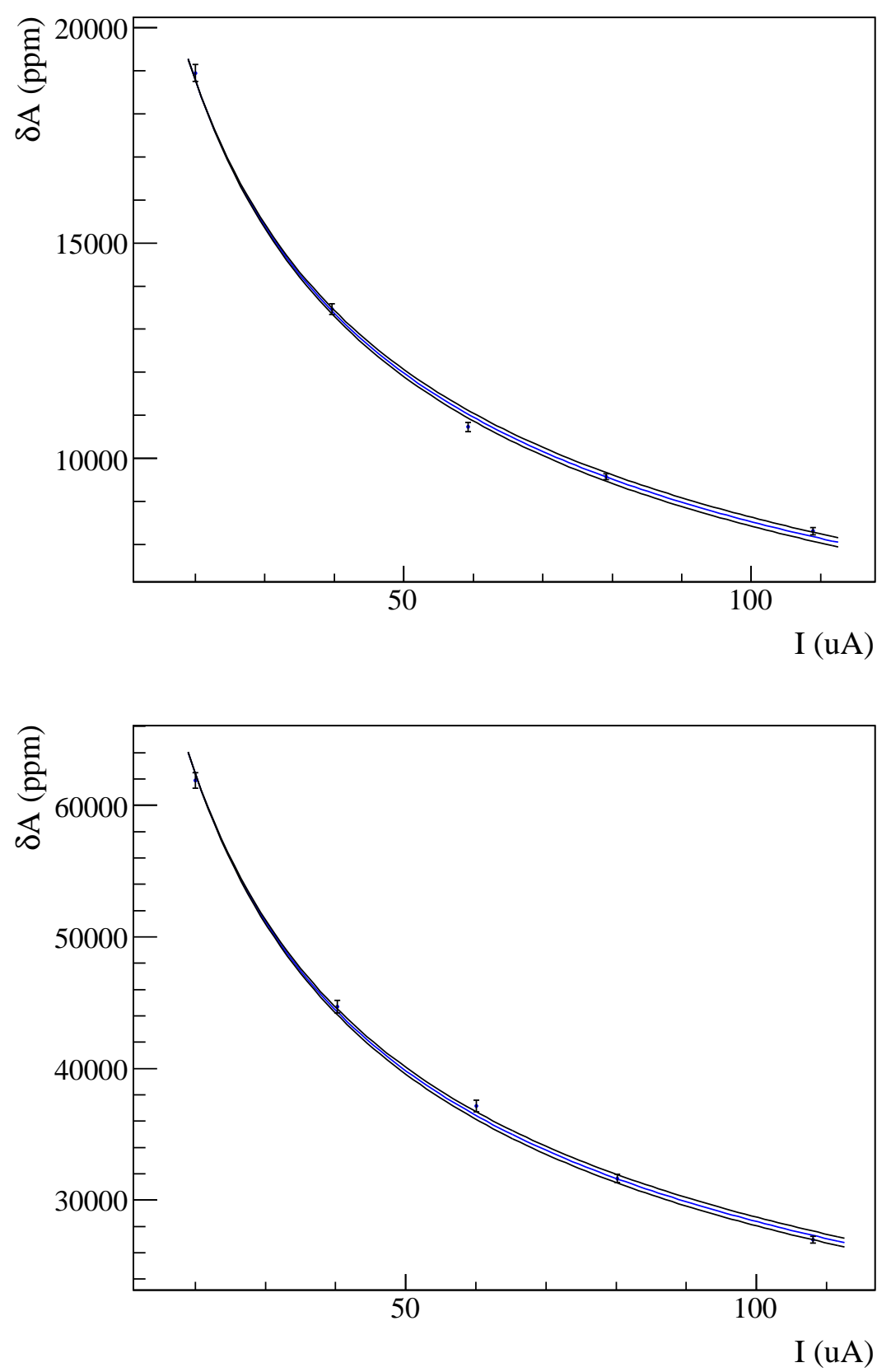

Figure 2.14: Measured standard deviation of the pair-wise asymmetries at various beam currents for DIS\# 1 (top) and \# 2 (bottom), with a $4 \times 4 \mathrm{~mm}^{2}$ square raster. The curves show results of fit $\delta A \propto I^{p_{0}}$ and its error. The fit results are $p_{0}=$ $0.4900 \pm 0.0076$ and $p_{0}=0.4897 \pm 0.0072$ for DIS\# 1 and $\# 2$ respectively. These results are in good agreement with pure counting statistics $(\delta A \propto \sqrt{I})$. 
of the pair-wise asymmetries, $\delta A$, taken at kinematics DIS \#2 for various raster sizes at two beam currents 100 and $115 \mu \mathrm{A}$. For each setting, the pair-wise asymmetry distribution was fitted using a Gaussian distribution where the fit result of the Gaussian width was taken as $\delta A$ and the uncertainty of the fit as the uncertainty of $\delta A$. Results in Fig. 2.13 were fitted with the functional form $p_{0} x^{p_{1}}+p_{2}$ where $x$ is the raster size in $\mathrm{mm}$. The parameter $p_{2}$ represents the purely statistical fluctuation that depends only on the beam current and not the raster size, while the term $p_{0} x^{p_{1}}$ is an empirical term that describes the size of local boiling. The figure shows that the broadening due to boiling effect, $p_{0} x^{p_{1}}$, is quite small above a $4 \times 4 \mathrm{~mm}^{2}$ raster size. Even for other kinematics with higher rate hence smaller standard deviation for the asymmetry, (such as kinematic DIS \#1 as shown in Fig. 2.14), the boiling effect did not contribute significantly to the uncertainty of the asymmetry measurement.

Figure 2.14 shows the measured standard deviation of the pair-wise asymmetries for various beam currents $I$ performed with a $4 \times 4 \mathrm{~mm}^{2}$ square raster. No significant deviation from pure counting statistics $(\propto \sqrt{I})$ is observed, indicating that boiling effects at the running condition of this experiment was negligible.

\subsection{High Resolution Spectrometers}

The core components of the Hall A experimental setup are two high resolution spectrometers [43], which are positioned on the left and right sides of the beamline and are referred to as the Left and the Right HRS (or L-HRS and R-HRS in short) respectively. The two spectrometers are almost identical in configuration and can be used for both independent and coincidence measurements. During PVDIS, the two HRS's were used independently, and depending on the running period, they might be set at the same or different kinematics (See Table 3.1). 
The basic layout of the spectrometer is shown in Figure 2.15. Each spectrometer can be generally divided into two parts: 1) the "Optics" part that consists of three quadrupoles and one dipole in a QQDQ configuration and acts like a set of optical lenses for focusing and bending the electron beam; 2) the detector package which contains various detector modules for particle detection and identification, as well as the accompanying electronics for data acquisition. The main design characteristics of the HRS is shown in Table 2.2.

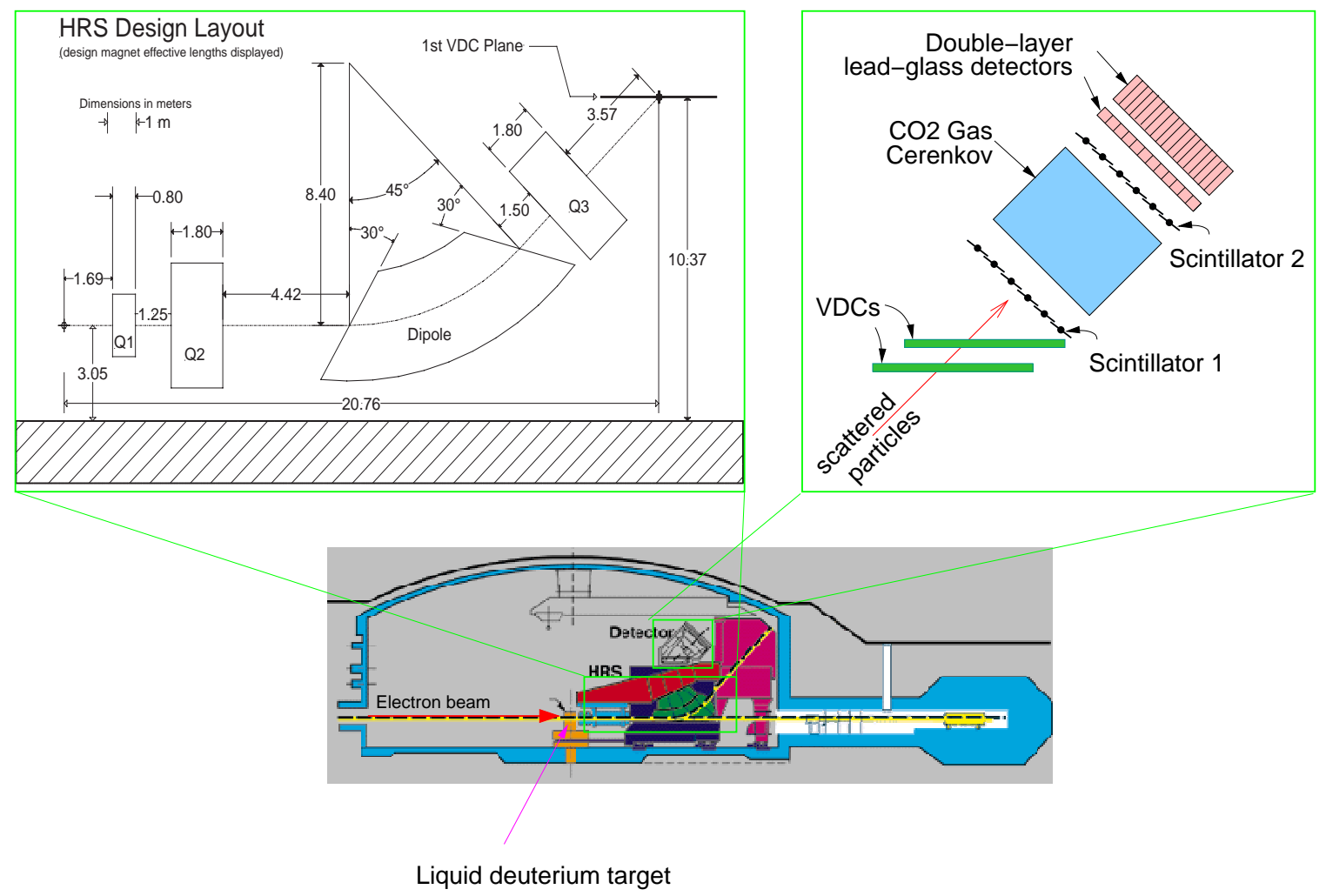

Figure 2.15: Layout of the HRS spectrometer.

\subsubsection{Optics Magnets}

A schematic view of the optics magnets is shown in the zoomed-in picture on the top left of Figure 2.15, with detailed dimension information marked. The vertical bending 


\begin{tabular}{|c|c|}
\hline Configuration & QQDQ Vertical bend \\
\hline Bending angle & $45^{\circ}$ \\
\hline Optical length & $23.4 \mathrm{~m}$ \\
\hline Momentum range & $0.3-4.0 \mathrm{GeV} / \mathrm{c}$ \\
\hline Momentum acceptance & $-4.5 \%<\delta \mathrm{p} / \mathrm{p}<+4.5 \%$ \\
\hline Momentum resolution & $1 \times 10^{-4}$ \\
\hline Dispersion at the focus (D) & $12.4 \mathrm{~m}$ \\
\hline Radial linear magnification (M) & -2.5 \\
\hline $\mathrm{D} / \mathrm{M}$ & 5.0 \\
\hline Angular range HRS-L & $12.5^{\circ}-150^{\circ}$ \\
\hline HRS-R & $12.5^{\circ}-130^{\circ}$ \\
\hline Angular acceptance: Horizontal & $\pm 30 \mathrm{mrad}$ \\
\hline Vertical & $\pm 60 \mathrm{mrad}$ \\
\hline Angular resolution : Horizontal & $0.5 \mathrm{mrad}$ \\
\hline Vertical & $1.0 \mathrm{mrad}$ \\
\hline Solid angle at $\delta \mathrm{p} / \mathrm{p}=0, \mathrm{y}_{0}=0$ & $6 \mathrm{msr}$ \\
\hline Transverse length acceptance & $\pm 5 \mathrm{~cm}$ \\
\hline Transverse position resolution & $1 \mathrm{~mm}$ \\
\hline
\end{tabular}

Table 2.2: Main design characteristics of the Hall A High Resolution Spectrometers.

of the electron beam is achieved by using a 6.6-meter-long dipole with focusing entrance and exit windows and additional focusing gradients inside. Two quadrupoles, named Q1 and Q2, were placed before the dipole to focus the beam in the dispersive (vertical) and transverse directions. After the beam is bent $45^{\circ}$ upward by the dipole, a third quadrupole Q3, which is identical to Q2, is used to transversely refocus the beam before it hits the detectors. With this design, the spectrometer can have a large angle and momentum acceptance with excellent resolutions, providing high accuracy for reconstructed kinematic variables.

All eight magnets contained in the two spectrometers are superconducting and cooled by a common cryogenic system that also cools the target. Each of the magnets has its own independent reservoir for the storage of helium and liquid nitrogen cryogens, as well as the control system consisting of cryogenic valves, liquid level devices, temperature sensors and pressure sensors. The magnets can be energized with 
currents over $1300 \mathrm{~A}$, producing magnetic fields up to $1.5 \mathrm{~T}$. The magnetic field in each dipole is measured and monitored using NMR probes, placed about $1.5 \mathrm{~m}$ inside the magnet entrance and provide very accurate field readings at the $10^{-5}$ level. Supplemental to the NMR probes, all magnets, including the quadupoles, are equipped with Hall probes and Gaussmeters for field monitoring. However, the Hall probes are only used as backups due to their lack of long-term stability and reproducibility. Therefore, the magnetic fields in the quadupoles are set based on their current settings. Due to the hysteresis effect, magnetic cycling is necessary for the quadupoles Q2 and Q3, which is done by always going to the nominal maximum current of 1600 A before setting their currents to the desired value. The hysteresis effect in Q1 is small enough that cycling is not required.

The three quadupoles and the detector elements are hung from or mounted on a box beam that is mounted on the top of the dipole, making their relative position constant regardless of the spectrometer azimuthal position in the horizontal plane. The structure of each spectrometer arm is supported by a rigid metal frame, which can be moved circularly around the Hall A center by a series of bogie-mounted conical wheels, allowing the spectrometer to be positioned at any azimuthal angle. Each of the wheels is driven by a servomotor through a gear reducer and is controlled by a Graphic User Interface (GUI) on the computer, making it possible to remotely move the spectrometer. However, during PVDIS, the wheel control was broken for a period when kinematic change were needed, therefore, PVDIS became the first experiment in the Hall A history that manually moved the spectrometer using drills to operate the wheels. 


\subsubsection{Detector Package}

Coming out of the last quadrupole Q3, particles reach the detector hut located on the top floor of the spectrometer (See Figure 2.15). The hut is made of a $10 \mathrm{~cm}$ thick steel frame with a $5 \mathrm{~cm}$ lead layer inside and a concrete layer outside to protect the detectors against radiation from all directions. It contains a package of detectors and related electronics to provide various functions in the characterization of charged particles passing through the spectrometer. During PVDIS, the detector packages on both HRS's had very similar configurations:

- A pair of Vertical Drift Chambers (VDCs) to provide tracking information;

- Two planes of scintillators to provide the basic triggers;

- A gas Cerenkov detector for particle identification (PID);

- A pair of lead glass counters for additional PID.

\subsubsection{Vertical Drift Chambers}

The Vertical Drift Chambers $[56,57]$ provide accurate measurements of the incident position and angle of charged particles at the spectrometer's focal plane, which, when reconstructed with the knowledge of the spectrometer optics, give precise information on the important kinematics variables at the target scattering point. There is a pair of VDCs in each of the spectrometers' detector hut, the schematic layout of which is shown in Figure 2.16. The two VDCs are laid horizontally and separated by a distance of $33.5 \mathrm{~cm}$, with the upper VDC shifted by another $33.5 \mathrm{~cm}$ in the dispersive direction to accommodate the $45^{\circ}$ angle of the nominal particle trajectory. Each VDC consists of two planes of wires in a standard UV configuration: the wires in each plane are perpendicular to each other and are oriented at an angle of $45^{\circ}$ with respect to 
the transverse and the dispersive directions. There are a total of 368 sense wires for each plane, spaced $4.24 \mathrm{~mm}$ apart and covering an active area of $2188 \mathrm{~mm} \times 288 \mathrm{~mm}$. The two wire planes are separated by $26 \mathrm{~mm}$ in distance.

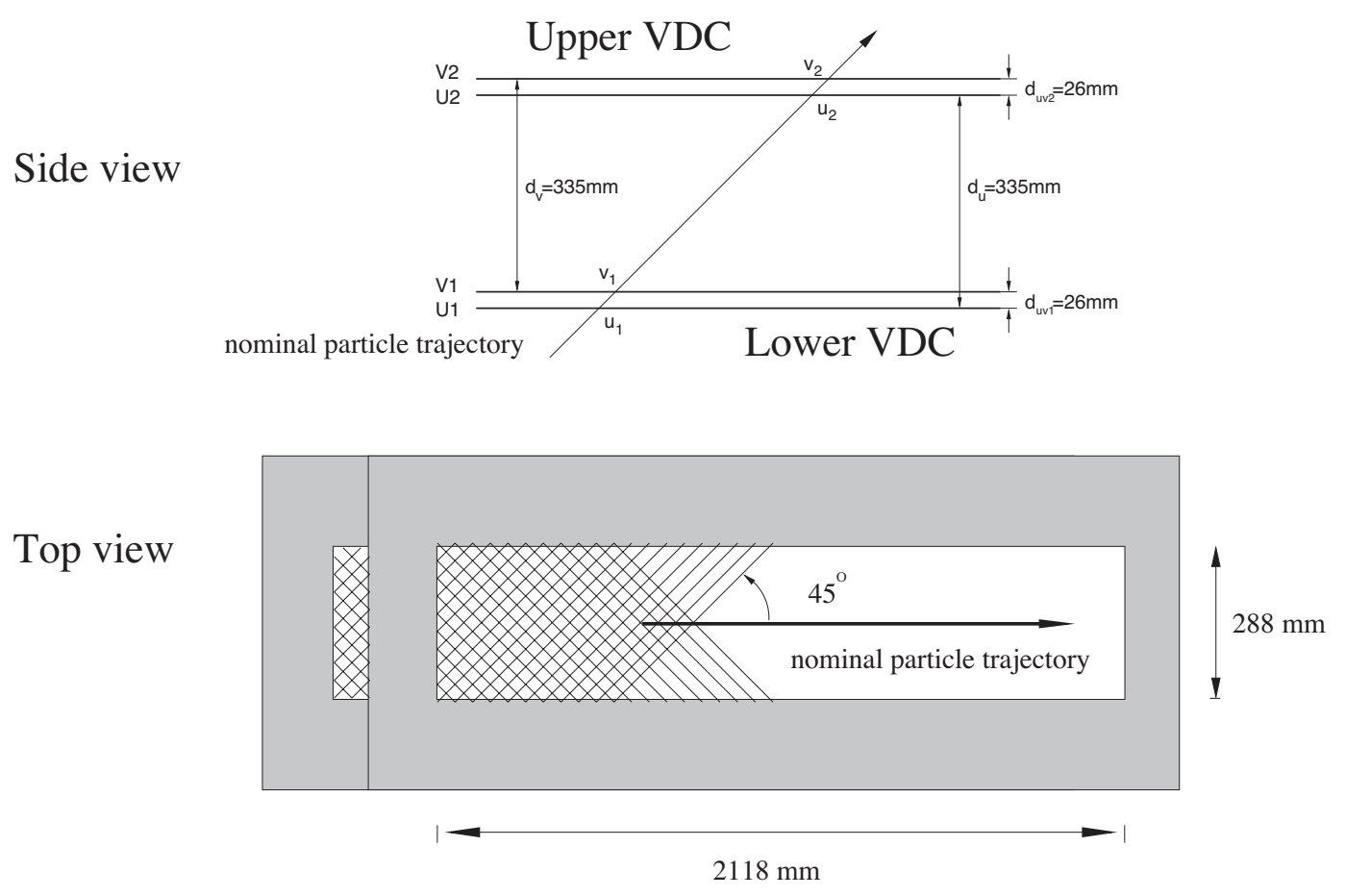

Figure 2.16: Schematic diagrams of the VDC configuration.

Each VDC has three gold-plated Mylar planes, one located between the U and the V planes and the other two on opposite sides. During operation, the Mylar planes are connected to a $-4 \mathrm{kV}$ high voltage while the wires are grounded. This produces an almost uniform electric field for particle drifting. The VDCs are filled with a mixture gas of $62 \%$ argon and $38 \%$ ethane $\left(\mathrm{C}_{2} \mathrm{H}_{6}\right)$, which flows at a rate of 10 liter per hour. When a charged particle passes through the chamber, it ionizes the gas and leaves behind a track of electrons and ions along its path. The ionized electrons accelerate to 
the sense wires along the path of least time, and wires fired by the electrons are read out by Time-to-Digital Converters (TDCs) operating in common stop mode. Using the drifting time obtained from the TDCs, together with the drift velocity of the electrons (approximately $50 \mu \mathrm{m} / \mathrm{ns}$ ), one can extract the distance from the particle trajectory to each fired wire. Combining the drift distances from all fired wire gives the full trajectory of the charged particle. Trajectories reconstructed this way have a position resolution of $\sigma_{x, y} \approx 100 \mu \mathrm{m}$ and an angle resolution of $\sigma_{\theta, \phi} \approx 0.5 \mathrm{mrad}$.

During PVDIS, the VDCs were turned off during production runs because they couldn't endure the high event rate generated. They were only turned on for optics study at low beam current, which happened about once a day.

\subsubsection{Scintillators}

When running with the standard HRS DAQ (Section 2.6.1), the triggering is provided by two scintillator planes, named $\mathrm{S} 1$ and $\mathrm{S} 2$, separated by a distance of $2 \mathrm{~m}$. Each plane is composed of six overlapping paddles made of thin plastic scintillators to minimize hadron absorption. For each paddle, the scintillation light is detected by two PMTs attached to the opposite ends of the paddle. The active area of scintillators is $29.5 \times 35.5 \mathrm{~cm}^{2}$ for plane $\mathrm{S} 1$ and $37.0 \times 54.0 \mathrm{~cm}^{2}$ for plane $\mathrm{S} 2$, and the time resolution for each plane is about $0.30 \mathrm{~ns}$. To form the trigger, a coincidence is taken between the two PMTs for each paddle, then these signals are combined together using a logic OR, resulting in one signal for each plane. Finally a trigger is generated by forming the logical AND between the S1 and the S2 signals. The trigger produced by the scintillators serves as the basic trigger for the HRS DAQ. 


\subsubsection{Gas Cerenkov}

The main background particles for PVDIS are pions resulting from photo-production. This makes the Gas Cerenkov detector a very powerful tool for PID because in principle pions produce no signal if they are below the threshold momentum when passing through the Gas Cerenkov. Therefore, a simple threshold cut on the Cerenkov signal can separate electrons from pions.

The operation of the Cerenkov detector is based on the Cerenkov effect: Cerenkov radiation is emitted when a high energy charged particle passes through a transparent material with a velocity faster than the velocity of light in that material, $c / n$. Here $c$ is the speed of light in vacuum and $n$ is the refractive index of that material. This threshold velocity corresponds to a threshold particle momentum required for Cerenkov light emission through the relation:

$$
p_{t h}=\frac{m v_{t h}}{\sqrt{1-\frac{v_{t h}^{2}}{c^{2}}}}=\frac{m \frac{c}{n}}{\sqrt{1-\frac{c^{2}}{n^{2} c^{2}}}} \approx \frac{m c}{\sqrt{n^{2}-1}}
$$

The threshold momentum depends on the particle mass and are different for electrons and pions. The HRS Cerenkov detectors are filled with $\mathrm{CO}_{2}$ gas at atmospheric pressure. The refraction index is $n=1.00041$, which translates into a threshold momentum of $0.017 \mathrm{GeV}$ for electrons and $4.8 \mathrm{GeV}$ for pions. Therefore within the HRS designed momentum range of $0.3 \sim 4.0 \mathrm{GeV} / \mathrm{c}$, only electrons can emit Cerenkov light and leave a signature in the detector.

The gas Cerenkov detectors are positioned between the S1 and the S2 scintillator planes in each HRS and have very similar structure. Each one is made of steel and measures about $1.5 \mathrm{~m}$ thick, with an entrance window of $250 \times 80 \mathrm{~cm}^{2}$. The emitted Cerenkov light is collected by mirrors mounted on two opposite sides of the entrance window, and is focused onto PMTs located on the sides of the detector. The PMTs 
are read out by ADCs and used for PID analysis.

\subsubsection{Lead Glass Counters}

HRS-L
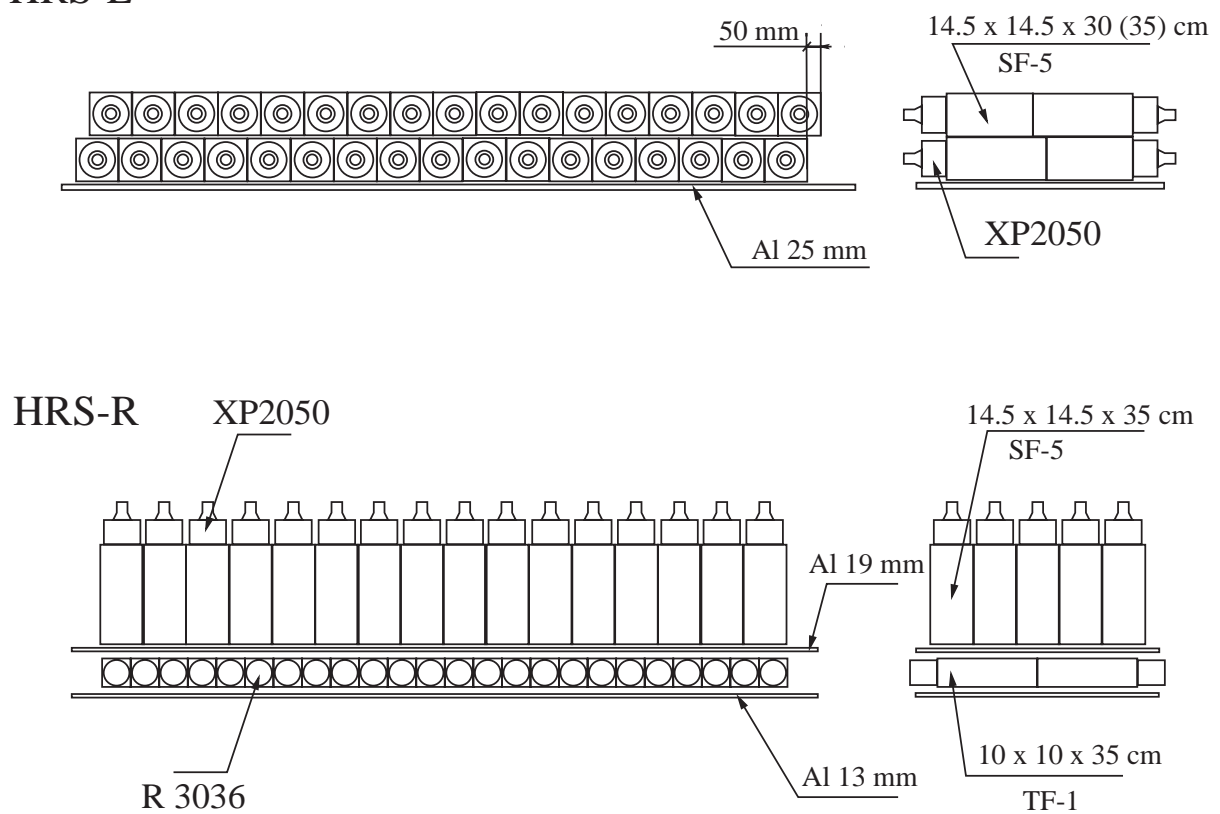

Figure 2.17: Configuration of the pion rejectors on the left HRS (top) and the preshower/shower detectors on the right HRS (bottom).

Lead glass counters provide additional particle identification. High energy particles deposit energy in lead-glass as they pass through and produce signals proportional to the energy deposited. Electrons tend to deposit more energy than pions because electromagnetic showers develop in the counters whereas hadronic showers do not due to the longer hadronic mean free path. Therefore the ADC signal spectrum in the lead glass counters are different for electrons and pions, which enable us to perform PID analysis and separate the two.

There are two layers of lead glass counters in each of the HRS with different configurations, as shown in Figure 2.17. On the Left HRS, the two layers are called "pion rejector I" and "pion rejector II" respectively. Each layer consists of 34 blocks, 
17 short and 17 long, forming a $2 \times 17$ array. All the blocks are oriented transversely with respect to the direction of incoming particles. On the right HRS, the first and the second layers are referred to as "preshower" and "shower" detectors. The preshower has a total of 48 blocks arranged in a $2 \times 24$ array that is again oriented transversely with respect to the direction of incoming particles. The shower has $5 \times 16$ blocks, and these blocks are oriented parallelly to the particles direction. Preshower and shower together are also called "total shower".

All lead glass counter blocks are coupled with PMTs and read out using ADCs. PID analysis based on lead glass is discussed in Section 3.8.

\subsection{Data Acquisition System}

The PVDIS experiment uses the CEBAF Online Data Acquisition (CODA) [58] system for data collection. CODA is a toolkit developed by the Jefferson Lab Data Acquisition Group and is specifically designed for nuclear experiments. It is composed of a set of software and hardware packages from which a data acquisition (DAQ) system can be constructed for monitoring, collection and storage of the data generated during experiments.

A typical DAQ system consists of several VME crates integrated by CODA. Each VME crate generally includes front-end electronics such as digitization devices (amplitude-to-digital converters (ADCs), time-to-digital converters (TDCs) and scalers), a Readout Controller (ROC, which is a single-board VME computer running VxWorks operating system) and Ethernet networks for communicating to CODA. A trigger supervisor (TS) is used to generate the trigger signal for all the VME crates and synchronize the operation of these crates. The Run Control interface of CODA runs on Linux workstations. It organizes all the pieces of data information coming 
from different VME crates and forms a compact structure of data stream which eventually gets written to a mass storage tape silo (MSS) for data storage. In addition, CODA also allows for insertion of data into the data stream from a slowly updating system called the Experimental Physics and Industrial Control System (EPICS). EPICS is used to record the information about the magnet status, beam properties as well as target cell variables, and it typically updates the data every few seconds.

During PVDIS, two separate DAQ systems were used, both running under the framework of CODA but with different configurations of electronics and triggering systems. The two DAQs served different purposes of data acquisition and ran in parallel throughout the whole experiment. Details of these two DAQs are described below.

\subsubsection{HRS DAQ}

One set of DAQ system is the standard DAQ system implemented in Hall A, referred to as the "HRS DAQ" so forth. It uses Fastbus TDCs and ADCs for recording the signal timing and amplitudes of different detectors, and scalers for counting some specific signals. The main trigger for the HRS DAQ is from the scintillator detector (Section 2.5.2.2). The HRS DAQ has high instrument deadtime due to the time needed for waiting for all the ADC data and recording them, so it can only process data up to a rate of about $4 \mathrm{kHz}$, which is significantly lower than our production rates. Therefore, the trigger rate of the HRS DAQ was heavily prescaled. Only a small fraction of all the triggers are recorded in the HRS data, so it wouldn't be appropriate to use the HRS DAQ for asymmetry measurements. Instead, it was mainly used for

monitoring the particle identification performance of the detectors, and calibration of the kinematics variables. 


\subsubsection{Parity DAQ}

One major challenge of the PVDIS experiment is to separate the scattered electrons from the pion background in the spectrometer and detector system. The separation could be done during off-line data analysis if all detailed information about the timing and energy of scattered particles was recorded, such as in the HRS data. However, as mentioned before, the HRS DAQ is only capable of recording data at low rates (4 $\mathrm{kHz}$ ), much lower than the rates produced in this experiment (several hundred $\mathrm{kHz}$ ). Therefore, in order to perform the particle identification accurately at very high event rates, a scaler-based counting DAQ system with hardware-based PID was customly designed and built from scratch.

This scaler-based counting DAQ, which will be referred to as the "PVDIS DAQ" hereafter, resides on the basis of the DAQ of the HAPPEX-III experiment [46]. It uses the same Trigger Supervisor system and CODA configuration as the HAPPEX DAQ. The main difference lies in the pre-processing of the detector signals to form particle (electron and pion) triggers, which were then counted by scalers. Scaler values were read out for every helicity window and recorded in the data stream, from which asymmetries can be calculated later on during data analysis. Detailed description of the PVDIS DAQ setup is presented bellow.

The PVDIS DAQ was designed to record data up to $600 \mathrm{kHz}$ with well understood deadtime and good PID performance at the hardware level. Inputs to the DAQ system are raw detector signals from the Scintillators, the gas Cerenkov detector and the double-layered lead glass counter (Section 2.5.2). The scintillator signals are first combined together and digitized to from a "T1" signal, which is also the main trigger of the HRS DAQ. Signals from the gas Cerenkov are also combined and digitized, producing a single "GC" signal as input to the DAQ. Signals from the lead glass counters were sent to the DAQ differently. In order to reduce the DAQ deadtime, 
the lead-glass blocks in both the preshower and the shower detectors were divided into 6 (8) groups for the Left (Right) HRS, with each group consisting typically eight blocks. This grouping mechanism is depicted in Figure 2.18. In order to maximize the electron detection efficiency, the position of each preshower group were aligned as much as possible with those of the corresponding shower group. On the Left HRS, adjacent groups in both preshower and shower had overlapping blocks, while for the Right HRS only preshower groups were overlapping. Signals from the overlapping blocks were split into two identical copies using passive splitters.

\section{Left HRS}

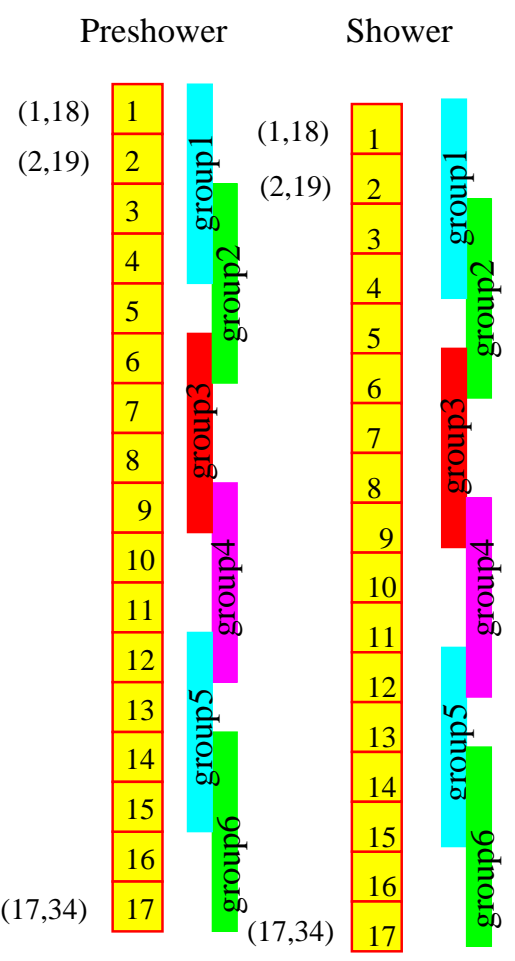

Right HRS

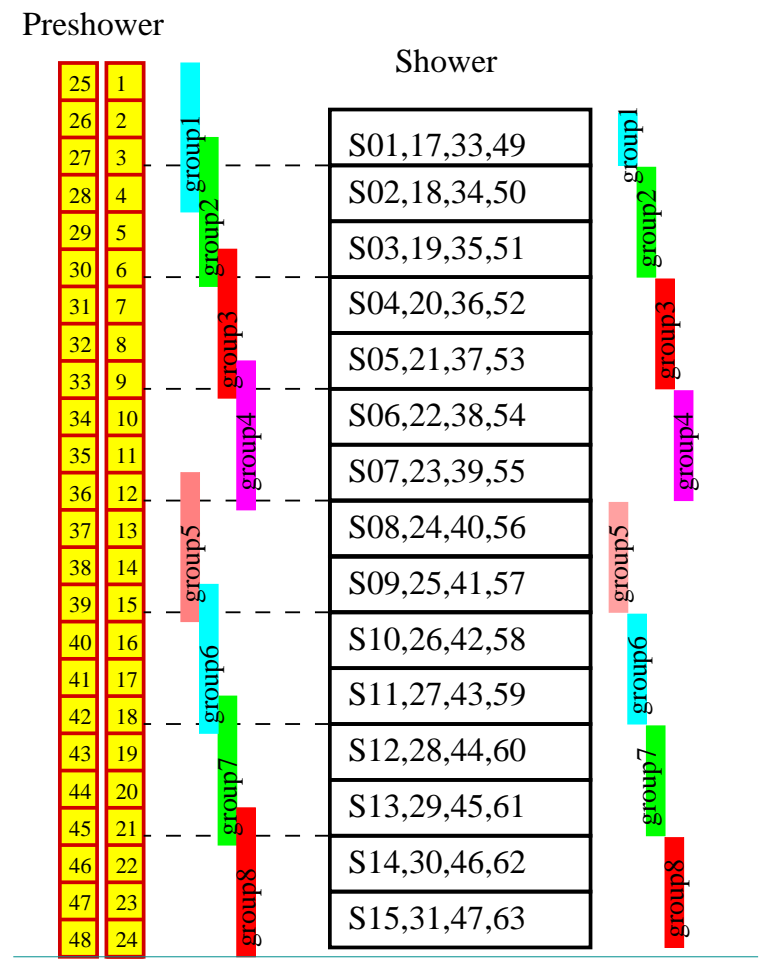

Figure 2.18: Grouping scheme (side-view) for the double-layer lead-glass detectors for the Left and the Right HRS. Scattered particles enter the detector from the left. The colored vertical bars represent the range of each group.

From the T1, the GC signals and the grouped lead glass counter signals, we can form the electron and pion triggers using various electronic modules with the proper 
logic. A schematic diagram of the DAQ electronics for the Right HRS is shown in Figure 2.19. Signals from lead glass blocks of each group were first added together using custom-made analog summing units called the "SUM8 module". The resulting groupwise preshower (PS) and shower (SS) signals and their sums, called total shower (TS) signals, were passed through discriminators with different thresholds. The discriminators perform hardware cuts on the energy deposits in the preshower and shower which separate electrons from pions. For electron triggers, logical ANDs of the PS discriminator and the TS discriminator outputs were used. For pions, low threshold discriminators on the TS signal alone were used. Additional PID was achieved by the "GATE" circuit, which combined the GC and the T1 signals. Each valid coincidence between GC and T1 would produce a 150-ns wide electron GATE signal that allowed an output to be formed by the logical AND modules from the preliminary electron triggers. Each valid T1 signal without the GC signal would produce a 150-ns wide pion GATE signal that allowed an output to be formed by the logical OR modules from the preliminary pion triggers. The outputs of the logical AND and OR modules are called group electron and pion triggers respectively. All six (eight) group electron or pion triggers were then ORed together to form the global electron or pion trigger for the Left (Right) HRS. All group and the global electron and pion triggers were counted using scalers.

In order to monitor the counting deadtime of the DAQ, two identical paths of electrons were constructed. The only difference between the two paths is in the PS and the TS discriminator output widths, set at 30 ns and 100 ns for the "narrow" and the "wide" paths, respectively. Detailed analysis of the DAQ deadtime will be presented in Section 3.7.

Full sampling of partial analog signals were done using Flash-ADCs (FADCs) at low rates intermittently during the experiment. For one group on the Left and 


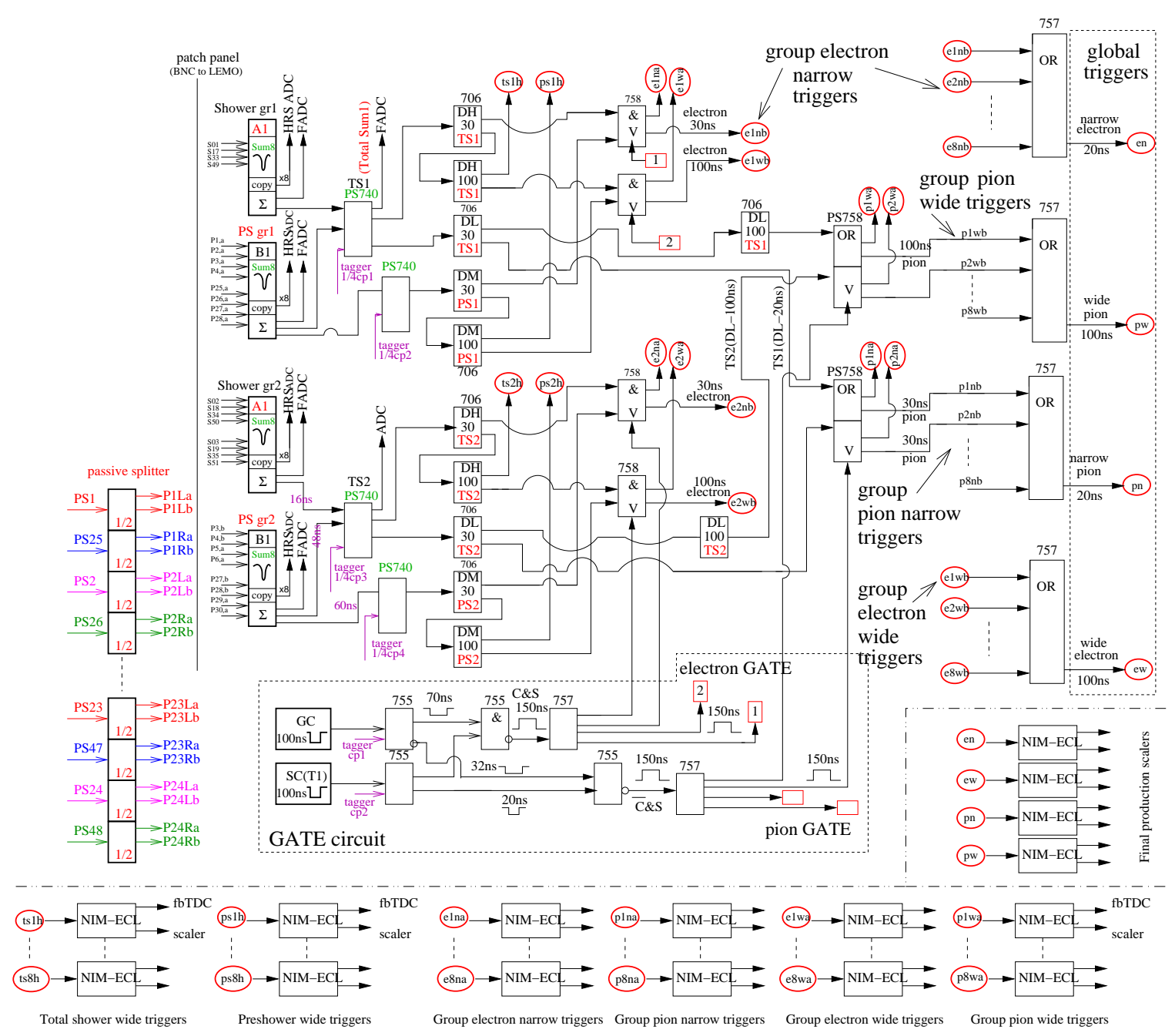

Figure 2.19: Schematic diagram of the trigger logic of the Parity DAQ. See the text for detailed explanation. 
one group on the Right HRS, the preshower and the shower SUM8 outputs, the intermediate logical signals of the DAQ, and the output electron and pion triggers were recorded. These FADC data provided a study of pileup effects to confirm the deadtime simulation and to provide the input parameters for the simulation, specifically the rise and fall times of the signals and their widths.

The SUM8 modules used for summing all lead-glass signals also served as fan-out modules, providing exact copies of the input PMT signals. These copies were sent to the standard HRS DAQ for calibration. During the experiment, data were collected at low rates using reduced beam currents with both DAQs functioning, such that a direct comparison of the two DAQs can be made. The vertical drift chambers were used during these low rate DAQ studies. Outputs from all discriminators, signals from the scintillator and the gas Cerenkov, and all electron and pion group and global triggers were sent to Fastbus TDCs (fbTDC) and were recorded in the standard DAQ. Data from these fbTDCs were used to align amplitude spectrum and timing of all signals. They also allowed the study of the Cerenkov and the lead-glass detector performance for the new DAQ.

Detailed analysis of the DAQ performance, such as deadtime, PID efficiency, and the statistical quality of the asymmetry measurement are provided in Chapter 3 and Reference [59]. 
3

\section{Data Analysis}

This chapter describes details of the data analysis which lead to the physics results in Chapter 4.

\subsection{Overview}

\subsubsection{Overview of Data Taking}

The experiment ran between October 26th and December 22nd, 2009. Data were taken first with a $6-\mathrm{GeV}$ beam at two deep inelastic scattering settings at $Q^{2}=1.085$ and $1.901(\mathrm{GeV} / c)^{2}$. These were the main production kinematics referred to as DIS\#1 and DIS\#2, respectively. Due to limitations in the spectrometer magnets, DIS\#1 was taken only on the Left HRS, while DIS\#2 was taken on both Left and Right HRS's. Data were taken at five additional nucleon resonance settings to provide inputs to electromagnetic radiative corrections. Two of the resonance settings (RES IV and V) were taken with the $6 \mathrm{GeV}$ beam on the Left HRS, between DIS\#1 and \#2, and three other resonance settings (RES I, II and III) were taken with a $4.8 \mathrm{GeV}$ beam at the end of the experiment, on either Left or Right HRS. Dedicated measurements for the 
beam transverse asymmetry - also called normal asymmetry $A_{n}$ - were carried out at

DIS \#1 and \#2 in which the beam spin was directed perpendicular to the scattering plane. An overview of the beam energy and spectrometer setting for each kinematics, the observed scattered electron rate measured by the DAQ and the observed ratio in $\pi^{-} / e$ rates are shown in Table 3.1 in the chronological order.

\begin{tabular}{c|c|c|c|c|c|c|c}
\hline HRS & Date & Kine\# & $E_{b}(\mathrm{GeV})$ & $\theta_{0}$ & $E_{0}^{\prime}(\mathrm{GeV})$ & $R_{e}(\mathrm{kHz})$ & $R_{\pi} / R_{e}$ \\
\hline \multirow{6}{*}{ Left } & $11 / 04-12 / 01 / 2009$ & DIS\#1 & 6.0674 & $12.9^{\circ}$ & 3.66 & $\approx 210$ & $\approx 0.5$ \\
& $12 / 01-12 / 02 / 2009$ & $A_{n}$ & 6.0674 & $12.9^{\circ}$ & 3.66 & $\approx 210$ & $\approx 0.5$ \\
& $12 / 02 / 2009$ & RES V & 6.0674 & $14^{\circ}$ & 3.66 & $\approx 130$ & $<0.7$ \\
& $12 / 03 / 2009$ & RES IV & 6.0674 & $15^{\circ}$ & 3.66 & $\approx 80$ & $<0.6$ \\
& $12 / 04-12 / 17 / 2009$ & DIS\#2 & 6.0674 & $20.0^{\circ}$ & 2.63 & $\approx 18$ & $\approx 3.3$ \\
& $12 / 17-12 / 19 / 2009$ & RES I & 4.8674 & $12.9^{\circ}$ & 4.0 & $\approx 300$ & $<0.25$ \\
& $12 / 19-12 / 22 / 2009$ & RES II & 4.8674 & $12.9^{\circ}$ & 3.55 & $\approx 600$ & $<0.25$ \\
\hline \multirow{5}{*}{ Right } & $11 / 04-12 / 01 / 2009$ & DIS\#2 & 6.0674 & $20.0^{\circ}$ & 2.63 & $\approx 18$ & $\approx 3.3$ \\
& $12 / 01-12 / 02 / 2009$ & $A_{n}$ & 6.0674 & $20.0^{\circ}$ & 2.63 & $\approx 18$ & $\approx 3.3$ \\
& $12 / 02-12 / 17 / 2009$ & DIS\#2 & 6.0674 & $20.0^{\circ}$ & 2.63 & $\approx 18$ & $\approx 3.3$ \\
& $12 / 17-12 / 22 / 2009$ & RES III & 4.8674 & $12.9^{\circ}$ & 3.1 & $\approx 400$ & $<0.4$ \\
\hline
\end{tabular}

Table 3.1: Overview of kinematic settings of the experiment and the observed scattered electron rate $R_{e}$ and the negatively charged pion to electron rate ratio $R_{\pi} / R_{e}$. The kinematics include the beam energy $E_{b}$, and the spectrometer central angle $\theta_{0}$ and central momentum $E_{0}^{\prime}$. Measurement of the transverse asymmetry $A_{n}$ was performed at the production DIS settings on December 1-2.

During PVDIS, the insertable half-wave plate (IHWP) was inserted into or retracted from the beam line after about every one day of smooth data taking. The reversal of the IHWP state flipped the sign of the physics asymmetry but left other false asymmetries unchanged. Therefore, averaging of the sign corrected asymmetry over different IHWP states cancels these false asymmetries. Each of the periods when the IHWP state was fixed is called a "slug", and a typical production slug of the DIS kinematics contain about one million "good" helicity pairs. 


\subsubsection{Overview of Data Analysis}

There are mainly two sets of raw data collected simultaneously as the experiment was running, by the HRS DAQ and the PVDIS DAQ respectively (Section 2.6). The HRS data is analyzed by the Hall A Analyzer [60], an analysis software written in $\mathrm{C}++$ utilizing ROOT [61] libraries. This data provides key information about the kinematics settings and particle identification performance of the DAQ. The parity data is where all the raw asymmetries are extracted from, using another analysis software called the Parity Analyzer (PAN) [62]. PAN is also written in $\mathrm{C}++$ based on the ROOT platform and preforms a variety of analyzing tasks:

- Load in raw data and the control file (also called the "database") for analysis. Decode the raw data and map them to different detectors and monitors according to the database.

- Perform pedestal subtraction for ADCs and scalers. The pedestal values are determined from calibration runs and are written into the database as input.

- Determine the pass/fail status of various cuts for each event based on raw beam parameters and the DAQ condition. The cuts are also defined in the database.

- Check and synchronize the helicity signal with events.

- Form raw asymmetries from pairs of helicity windows.

After the raw asymmetry is extracted from the PAN analysis, various systematic corrections need to be considered and applied in order to get the physical asymmetry.

The extraction of the raw asymmetry from the PAN analysis is presented in Section 3.2. In order to get the physical asymmetry, various systematic corrections need to be considered and applied to the raw asymmetry. The corrections due to fluctuations in the beam intensity, position, angle and energy is described first (Section 3.4). 
The beam polarization is a major correction to the asymmetry and is presented the next (Section 3.6). Two other systematics specifically related to the DAQ system, the deadtime and particle identification efficiencies, are then studied and discussed in detail (Section 3.7 and Section 3.8). Calibrations of the beam position and HRS optics are crucial for evaluation of the event kinematics (section 3.9), and a full scale simulation of the HRS transport functions were carried out to confirm our understanding of kinematics resulting from these calibrations (section 3.10). Radiative corrections due to energy losses of the incident and the scattered electrons will be presented (section 3.11), followed by corrections due to the higher-order $\gamma \gamma$ box diagrams (section 3.11.3). Lastly, corrections to the measured asymmetries due to various background will be presented in detail (section 3.12).

The analysis flow described above is performed on the global electron trigger, and is carried out in the same fashion for both the DIS kinematics and the resonance kinematics. In addition, further analysis on the resonance data using group triggers is presented in Section 3.13

To prevent human bias during the data analysis, a "blinding" method was adopted to hide the true asymmetry value. A blinding factor, which was generated randomly at the beginning of data collection, was added to the raw asymmetry during the analysis. The blinding factor is significantly larger than the expected statistical uncertainty of the raw asymmetry and is not revealed until all the analysis tasks were finalized. Therefore, this method prevents any direct comparison of the measured asymmetry with theoretically calculated values during the analysis. The blinding factor was removed at the very end of data analysis when the group was ready to release the asymmetry results to public. 


\subsection{Raw Asymmetry}

\subsection{Forming Raw Asymmetries}

The integrated response of each beam monitor was digitized and recorded for each 33 msec window, while the scattered electrons were counted by the DAQ. For each window pair $i$, the pair-wise raw electron cross section asymmetry $A^{\text {raw }}$ in each HRS was computed from the the DAQ counts $c^{+(-)}$normalized to the integrated beam intensity $I^{+(-)}$in the positive (negative) helicity window:

$$
A_{i}^{\mathrm{raw}}=\left(\frac{\frac{c_{i}^{+}}{I_{i}^{+}-\frac{c_{i}^{-}}{I_{i}^{-}}}}{\frac{c_{i}^{+}}{I_{i}^{+}}+\frac{c_{i}^{-}}{I_{i}^{-}}}\right) \text {. }
$$

With a negligible beam intensity noise, the statistical uncertainty is

$$
\delta A_{i, \mathrm{stat}}^{\mathrm{raw}}=\sqrt{\frac{1}{c_{i}^{+}+c_{i}^{-}}} .
$$

If a total of $n$ window pairs have been collected, the raw measured asymmetry $A^{\text {raw }}$ was formed by taking the statistical average of $A_{i}^{\text {raw }}$ :

$$
A^{\mathrm{raw}}=\left\langle A_{i}^{\mathrm{raw}}\right\rangle \equiv \frac{\sum_{i=1}^{n} A_{i}^{\mathrm{raw}} /\left(\delta A_{i \text { stat }}^{\mathrm{raw}}\right)^{2}}{\sum_{i=1}^{n} 1 /\left(\delta A_{i, \mathrm{stat}}^{\mathrm{raw}}\right)^{2}},
$$

and its statistical uncertainty is

$$
\delta A_{\mathrm{stat}}^{\mathrm{raw}}=\sqrt{\frac{1}{N^{+}+N^{-}}} \approx \frac{\delta A_{i, \mathrm{stat}}^{\mathrm{raw}}}{\sqrt{n}},
$$

where $N^{ \pm}=\sum_{i=1}^{n} c_{i}^{ \pm}$refer to the total electron counts from the $n$ window pairs and the approximation is valid if the beam current remains stable. In reality, the 
uncertainty of $A^{\text {raw }}$ could be widened by helicity-dependent fluctuations in the beam intensity and other noise. This is discussed in general as follows:

For any experimentally measured quantity $M$, such as detector rate, beam intensity, or beam position, two key parameters describe the quality of the measurement: the first is $\delta\left(M^{+}-M^{-}\right)$, the size of the relative pair-to-pair fluctuations in the helicity difference $M^{+}-M^{-}$, which ideally is affected only by statistical fluctuations in the measured quantity; the second is $\epsilon\left(M^{+}-M^{-}\right)$, the relative accuracy with which the window pair difference $M^{+}-M^{-}$can be measured compared to the true value, which is dominated by instrumentation noise. If $\epsilon\left(M^{+}-M^{-}\right)$is large enough, it would show up as a non-statistical contribution to $\delta\left(M^{+}-M^{-}\right)$so that the latter is no longer dominated by counting statistics. An increased uncertainty of this kind is difficult to understand, and it implies that the desired precision cannot be met within the expected amount of time calculated using statistical uncertainty alone. Therefore, an important criteria for a successful asymmetry measurement is $\epsilon\left(M^{+}-M^{-}\right) \ll \delta\left(M^{+}-M^{-}\right)$, which minimizes the run time and ensures that the main source of the uncertainty is the well-understood statistical fluctuation.

The beam properties and the noise due to the liquid deuterium target are carefully monitored (Section 2.3.4 and 2.4) to make sure that the $\epsilon\left(c^{+}-c^{-}\right.$) is negligible compared to the statistical fluctuation. Additional corrections to account for the false asymmetries due to helicity correlated beam fluctuations are also applied (Section 3.4).

\subsubsection{Data Selection}

Many kinds of systematic errors and fluctuations can compromise the statistical quality and the accuracy of the data. To insure that only good quality data are gathered for extraction of physical asymmetry, various cuts were used for data selection. These 
cuts can be generally categorized into two different kinds:

1) Cuts that are imposed helicity by helicty based on the information recorded by the parity DAQ and analyzed by PAN. These cuts are defined in the control file/database of PAN and are automatically applied during the PAN analysis. They include the following:

- Low Beam Cut: This cut gets rid of running periods with no beam or significantly lower beam current, such as beam trips and ramps, background runs, and low current runs which will make the asymmetry width much bigger. A beam current monitor (BCM) signal of 21000 , which corresponds to roughly 70 $\mu \mathrm{A}$, is used as the low beam cutoff value. To avoid any instabilities during beam trips, 10 events before the beam trip and 40 events after the beam is recovered are also cut out.

- Beam Current Burp Cut: This cut kicks in when there are dramatic fluctuations in the beam intensity. Events with over $2 \%$ of sudden change in beam intensity are cut out, as well as 10 events before and 40 events after.

- Beam Position Burp Cuts: These cuts account for large fluctuations in beam positions and are applied on the beam position monitor (BPM) signals. Events with beam excursions higher than $200 \mathrm{~nm}$ are cut out, as well as 50 events both before and after.

- Monitor Saturation Cut: One of the beam position monitors, bpm12, might be saturated at times during the experiment. This cut is then specifically designed to cut out events with bpm12 signal higher than 132000, as well as 30 events both before and after.

- ADCX DAC Burp Cut: This cut gets rid of the erroneous signals for the 18-bit 
ADCs, referred to as ADCX. These errors are usually caused by large jumps in the DAC values.

- Event Sequence: As described in Section 2.3.1, the beam helicity signal is generated using a psuedo-random algorithm. This same algorithm is also implemented in PAN for predicting the helicity state. If the readout helicity from the DAQ doesn't agree with the predicted helicity, the event will be cut.

- Pair Sequence: When forming the asymmetry using a pair of events, the helicity of the two events should be of opposite sign. When this is not the case, that pair of events will be cut, as well as 25 events before and after.

- ADCX DAC Burp Cut: This cut gets rid of the erroneous signals for the 18-bit ADCs (referred to as ADCX). These errors are usually caused by large jumps in the DAC values.

- ADCX Bad: Any other data that might have been corrupted by internal ADC errors on the 18-bit ADCs. These errors are rare and are usually caused by miscommunication between the DAQ system and an individual electronics crate.

An additional cut, called the "ok_cut", is defined for each helicity pair when the raw asymmetry is formed. It is set to be true when both windows of the helicity pair pass all cuts defined above.

2) Longer-term cuts that are imposed over a specific running period, for example, when the equipment malfunctioned. These refer specifically to the malfunctions that don't have representative data recorded in the parity data stream, therefore these cuts cannot be determined during the PAN analysis. Instead, such cuts are applied manually during post-PAN analysis based on experimetal log entries recorded by the shift crew. These malfunctions include the following: 
- HRS magnets failure: The superconducting magnets of the HRS may lose power during the experiment, resulting in a non-functional HRS and false data that should be rejected. Since the two HRS ran independently during PVDIS, we discard only the data for the non-functional HRS while keeping that of the functioning one.

- DAQ malfunction: Some of the electronic modules of the DAQ system might stop functioning properly as the experiment went on. One such example is the ANDing modules used for forming the electron trigger by taking the coincidence between the preshower and shower signals, which stopped working at a late time during the experiment. Those modules were swapped by good ones as soon as the problem was spotted and the corresponding data were cut out.

- DAQ mis-setting: Sometimes the DAQ system may not behave as desired, not due to the malfunction of electronics, but the issue that some modules are not properly set. One of such examples happened during the kinematics change from DIS \#1 to DIS \#2, when the momentum of detected electrons changed while the threshold of the discriminators were not adjusted accordingly.

The DAQ related problems usually only existed for short periods of time as the data quality was constantly monitored by on-line analysis, and once a problem was found, it got addressed quickly.

With all the data selection cuts applied, a total of $1.02 \times 10^{7}$ and $2.5 \times 10^{7}$ beam helicity pairs were selected for the two main kinematics DIS \#1 and DIS \#2 respectively, and the statistical precision achieved were roughly $3 \%$ and $4 \%$. 

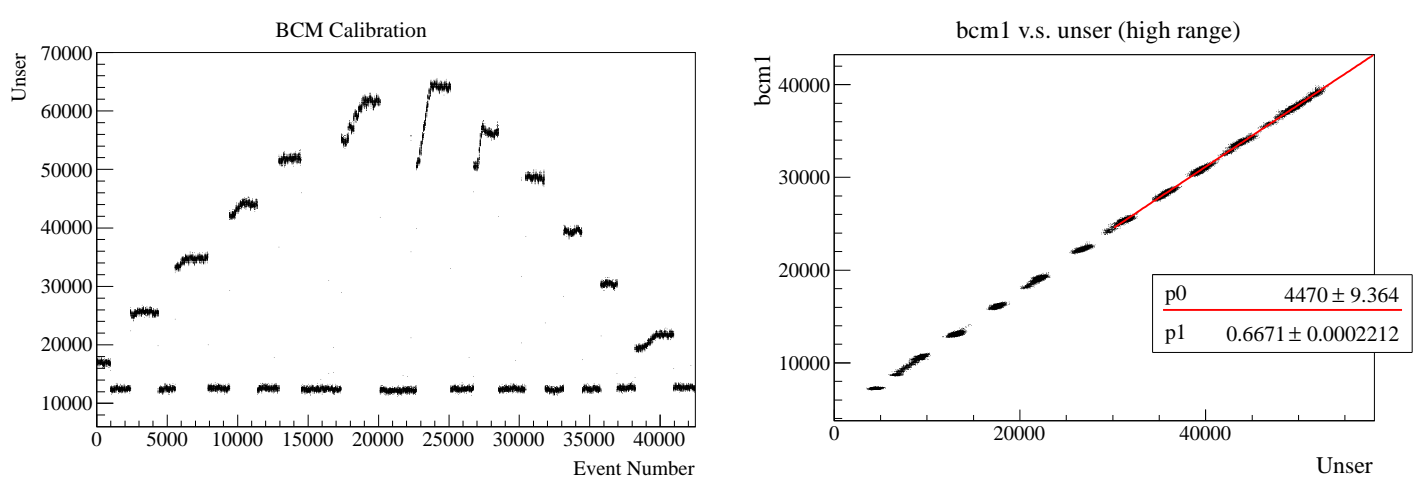

Figure 3.1: Illustration of the BCM calibration. Left: raw ADC readings for the Unser monitor plotted versus event number during the BCM calibration procedure; Right: raw ADC readings for bcm1 plotted versus that for the Unser monitor during $\mathrm{BCM}$ calibration. The red line shows the linear fit bcm $1=\mathrm{p} 0+\mathrm{p} 1 \times$ Unser. The fit is only performed with data at high current. The fitting result p0 represents the pedestal value of the bcm1 ADC reading.

\subsubsection{BCM Calibration}

The beam intensity used as the normalization factor in Equation 3.3.1 was measured by beam current monitors(BCMs) (Section 2.3.3.1). For PVDIS, the value of "bcm1", corresponding to the ADC reading of the upstream BCM with gain x1, was used as the normalization factor. The pedestal of the ADC needs to be properly calibrated before the ADC data can be used. This is done by designated calibration runs using the Unser monitor as a reference because it provides linear and absolute measurements of the beam current. During BCM calibrations, the beam current was ramped up from zero to its maximum $(\sim 120 \mu \mathrm{A})$ by a series of steps, with each step lasting for about 1 minute and the step spacing to be roughly $10 \mu \mathrm{A}$. Between each two adjacent steps, the beam was turned off for about 1 minute in order to account for the baseline drift of the Unser monitor. After reaching $120 \mu \mathrm{A}$, the beam current was then ramped down in the same fashion to complete the procedure. To extract the pedestal, the bcm1 ADC reading was plotted versus the Unser reading, as shown 
in Figure 3.1 and a linear fit was performed, the offset of which gave the pedestal value. Only data from the high current range, where our production data was taken, was included in the linear fit to avoid any ADC non-linearity at low currents. The pedestal value obtained from the BCM calibration was then written into the database for the production runs.

\subsection{False Asymmetry and Corrections}

\subsubsection{Charge Asymmetry}

With the charge feedback mechanism (Section 2.3.3.2) running throughout the experiment, the charge asymmetry was controlled to be very small (typically below 1 ppm). Besides, the normalization step (the " $1 / I$ " factor in Equation 3.3.1) performed before forming the asymmetry should, to the first order, cancel the contribution of charge asymmetry to the physical asymmetry. Therefore, it's not necessary to apply additional corrections to $A_{\text {raw }}$ to account for the charge asymmetry.

\subsubsection{Helicity Correlated Beam Fluctuations}

In the following we consider corrections from helicity-correlations in the beam. First, it is worth noting that for other parity-violating experiments carried out around the same time in Hall A of JLab Ref. [17] and [20], both the measured asymmetries and their statistical uncertainties were smaller by 1-2 orders of magnitude than what

were measured in the PVDIS experiment. For those experiments, uncertainties from helicity-correlations in the beam were already negligible compared to the statistical uncertainties of the measured asymmetries. Therefore beam corrections were not expected to contribute noticeably to the PVDIS experiment. 
When forming raw asymmetries, loose requirements were imposed on the beam quality: Periods with beam intensity, position or energy instability were rejected, removing about $25 \%$ of the total data sample. The dominant source of noise due to the beam arose from fluctuations in the beam position, angle, and energy, which are monitored by five BPMs: BPM4ax, BPM4ay, BPM4bx, BPM4by and BPM12x. These beam-related corrections (bc) can be parametrized as:

$$
\left(A^{\mathrm{bc}, \mathrm{raw}}\right)=A^{\mathrm{raw}}-\sum_{i}\left[\beta_{i}\left(\Delta M_{i}\right)\right]
$$

Here $\Delta M_{i}$ are the beam position differences measured by the BPMs and $\beta_{i} \equiv \partial \sigma / \partial M_{i}$ are the normalized detector sensitivities to $M_{i}$. The measurements of $\Delta M_{i}$ throughout the experiment is plotted in Figure 3.2. The sensitivities $\beta_{i}$ can be obtained using two methods: the beam modulation method and the regression method, which are described as following.

\subsubsection{Beam Modulation (Dithering)}

The beam modulation procedure (also called "dithering") is described in Section 2.3.4.2. Seven air-core corrector coils in the Hall A beamline upstream of the dispersive arc are used to modulate the beam positions and angles, and a vernier at the accelerator's South Linac is used to modulate the beam energy. The resulting variations in the five BPMs as well as variations in the detector asymmetry can be extracted from data. By studying how each of the BPMs responds to the modulation of each individual coils,

defined as $\partial M_{i} / \partial C_{j}$ ( $j$ is the index of coils), and also how the detector asymmetry changes accordingly, defined as $\partial \sigma / \partial C_{j}$, one can determine the detector sensitivities $\beta_{i}$ by solving the matrix inversion problem:

$$
B=D M^{-1}
$$



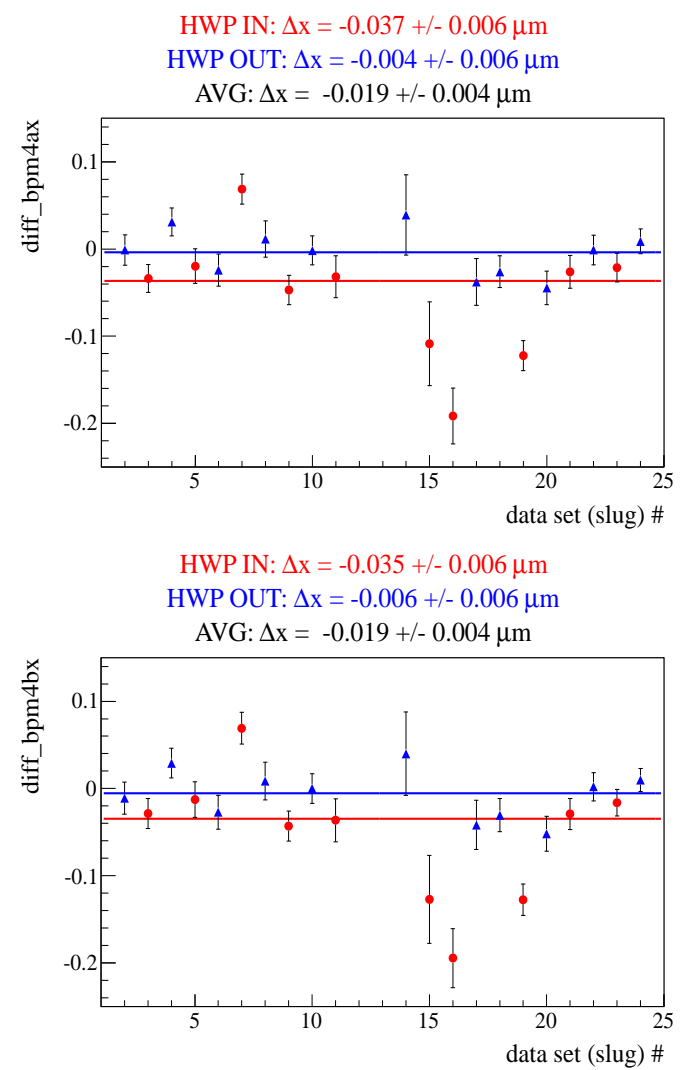

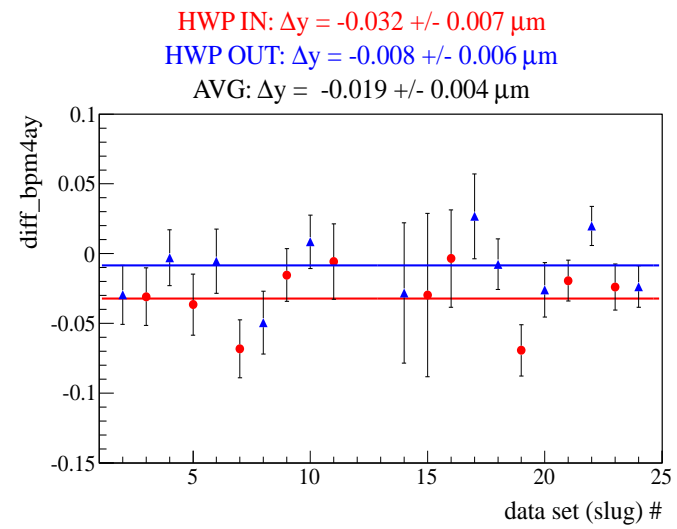

HWP IN: $\Delta y=-0.037+/-0.007 \mu \mathrm{m}$ HWP OUT: $\Delta \mathrm{y}=-0.009+/-0.006 \mu \mathrm{m}$ AVG: $\Delta \mathrm{y}=-0.022+/-0.005 \mu \mathrm{m}$

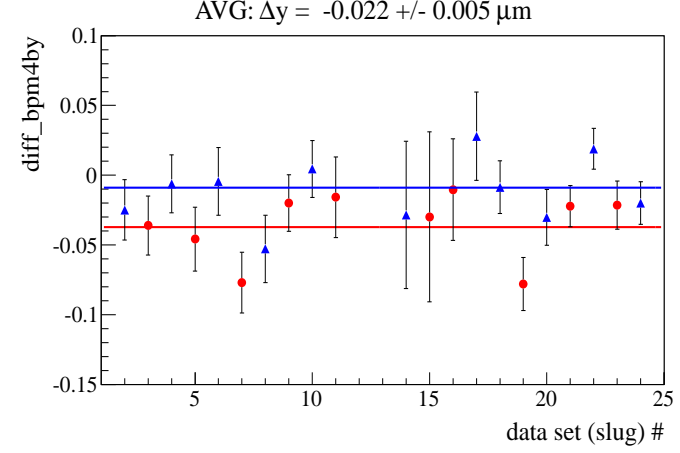

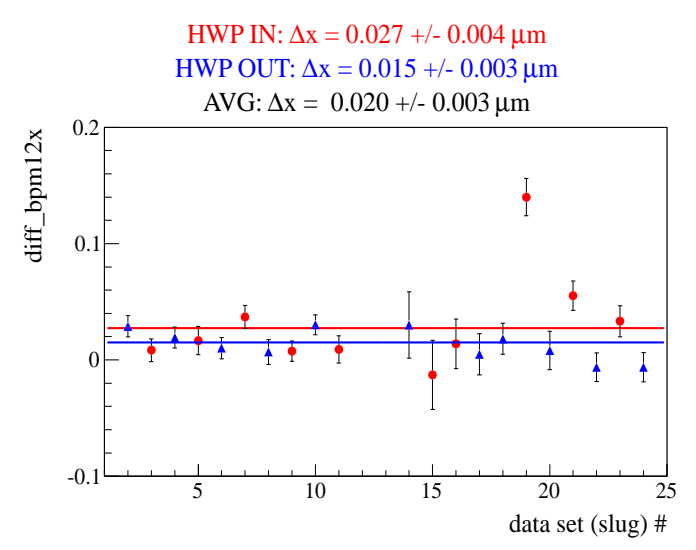

Figure 3.2: Beam position differences $\Delta M_{i}$ measured by the five BPMs throughout the PVDIS experiment, plotted versus the slug number. 
where the matrices are defined as:

$$
\begin{aligned}
D & =\sum_{j}\left(\frac{\partial \sigma}{\partial C_{j}} \frac{\partial M_{k}}{\partial C_{j}}\right) / \sigma^{2} \\
M & =\sum_{j}\left(\frac{\partial M_{i}}{\partial C_{j}} \frac{\partial M_{k}}{\partial C_{j}}\right) / \sigma^{2} \\
B & =\frac{\partial \sigma}{\partial M_{i}}
\end{aligned}
$$

With the sensitivities $\beta_{i}$ and beam motion differences $\Delta M_{i}$ in hand, the beam corrections can be calculated according to Equation 3.4.1. The corrections are then applied on each helicity pair. The final results of the beam corrections averaged over all the good helicity pairs are summarized in Table 3.2. Because the modulation periods represent quality data, they were included in the production data sample with the appropriate corrections made.

\subsubsection{Linear Regression}

The other way of applying beam corrections to the raw asymmetry is called the "regression" method. Instead of using modulation coils to intentionally move the beam around to study the sensitivities $\beta$, the regression method uses the "natural" motion of the beam. Therefore, all the data samples are useful for regression analysis. The sensitivities can be determined using a linear $\chi^{2}$ minimization algorithm, the detailed formalism of which is described in [63]. Both the dithering and the regression analyzes were performed on the PVDIS data. Eventually the dithering corrected asymmetry was the one we choose to apply systematic corrections and extract the physical asymmetry, while the regression analysis provides important cross-check on the dithering method. 


\begin{tabular}{|c|c|c|c|c|c|c|c|c|}
\hline \multicolumn{3}{|c|}{ Monitor } & BPM4AX & $\overline{\mathrm{BPM} 4 \mathrm{AY}}$ & 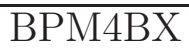 & 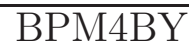 & $\overline{\mathrm{BPM} 12 \mathrm{X}}$ & Total \\
\hline \multirow{16}{*}{ 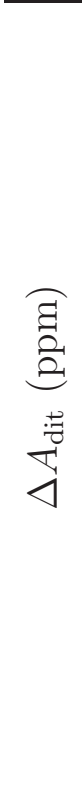 } & \multirow{2}{*}{ Left DIS\#1 } & $\bar{n}$ & 0.173 & 0.001 & -0.152 & -0.028 & 0.000 & -0.006 \\
\hline & & $\mathrm{W}$ & 0.179 & -0.010 & -0.159 & -0.020 & 0.000 & -0.010 \\
\hline & \multirow{2}{*}{ Left DIS\#2 } & $\mathrm{n}$ & 0.513 & 0.286 & -0.386 & -0.262 & 0.024 & 0.193 \\
\hline & & $\mathrm{W}$ & 0.569 & 0.262 & -0.430 & -0.243 & 0.022 & 0.180 \\
\hline & \multirow{2}{*}{ Right DIS\#2 } & $\mathrm{n}$ & -0.172 & -0.021 & 0.226 & -0.008 & -0.003 & 0.022 \\
\hline & & $\mathrm{W}$ & -0.182 & -0.027 & 0.237 & -0.003 & -0.003 & 0.022 \\
\hline & \multirow{2}{*}{ RES I } & $\bar{n}$ & -0.175 & 0.230 & 0.369 & -0.139 & -0.010 & 0.275 \\
\hline & & $\mathrm{w}$ & -0.178 & 0.224 & 0.375 & -0.133 & -0.011 & 0.277 \\
\hline & \multirow{2}{*}{ RES II } & $\mathrm{n}$ & 0.313 & 0.096 & -0.568 & $\begin{array}{l}-0.132 \\
\end{array}$ & 0.045 & -0.246 \\
\hline & & $\mathrm{w}$ & 0.320 & 0.107 & -0.582 & -0.143 & 0.045 & -0.253 \\
\hline & \multirow{2}{*}{ RES III } & $\bar{n}$ & -0.013 & 0.047 & 0.020 & -0.038 & -0.005 & 0.011 \\
\hline & & $\mathrm{w}$ & 0.000 & 0.046 & -0.005 & -0.037 & -0.005 & -0.001 \\
\hline & \multirow{2}{*}{ RES IV } & $\mathrm{n}$ & -1.004 & 0.328 & 1.398 & -0.235 & 0.002 & 0.489 \\
\hline & & $\mathrm{W}$ & -1.192 & 0.328 & 1.596 & -0.250 & 0.003 & 0.485 \\
\hline & \multirow{2}{*}{ RES V } & $\mathrm{n}$ & -3.708 & 0.400 & 4.745 & -0.265 & -0.035 & 1.146 \\
\hline & & $\mathrm{w}$ & -3.631 & 0.317 & 4.603 & -0.183 & -0.036 & 1.070 \\
\hline
\end{tabular}

Table 3.2: Corrections to asymmetries evaluated using the dithering method, $\Delta A_{\text {dit }}$. The "n" and "w" means "narrow" and "wide", and they refer to the DAQ trigger type (Section 2.6.2). The corrections were applied as $A_{\text {dit }}^{\text {bcraw }}=A^{\text {raw }}-\Delta A_{\text {dit }}$ [Equation 3.4.1]. 
The measured raw asymmetries $A^{\text {raw }}$ are shown in Table 3.3 along with the cor-

rected asymmetries based on both dithering and regression methods, $A_{\mathrm{dit}}^{\mathrm{bc}, \mathrm{raw}}$ and $A_{\text {reg }}^{\text {bcraw }}$. Differences between the two corrected asymmetries are also shown and these were used as the uncertainty in the beam corrections.

\subsection{Statistical Quality of Data}

Before proceeding to investigate further systematic corrections to be applied to the raw asymmetry, it is appropriate to first check the statistical quality of the data to make sure that the cuts and beam corrections performed so far, which was applied event by event or pair by pair, did not distort the data in any way. This check can be done using the pair-wise "pull" plot, defined as:

$$
p_{i} \equiv \frac{A_{i}-\bar{A}}{\delta A_{i}}, \text { where } \delta A_{i}=\frac{1}{\sqrt{C_{i}^{R}+C_{i}^{L}}}
$$

$A_{i}$ is the asymmetry extracted from the $i$-th beam helicity pair and $\bar{A}$ is the asymmetry averaged over all pairs. $\delta A_{i}$ is the statistical uncertainty of $A_{i}$ with $C_{i}{ }^{R(L)}$ the event count from the right (left) helicity window of the pair. The pull plots for different kinematics are shown in Figure 3.3. One can see that the pull spectra agree with the Gaussian distribution up to five orders of magnitude, as expected from purely statistical fluctuations. 


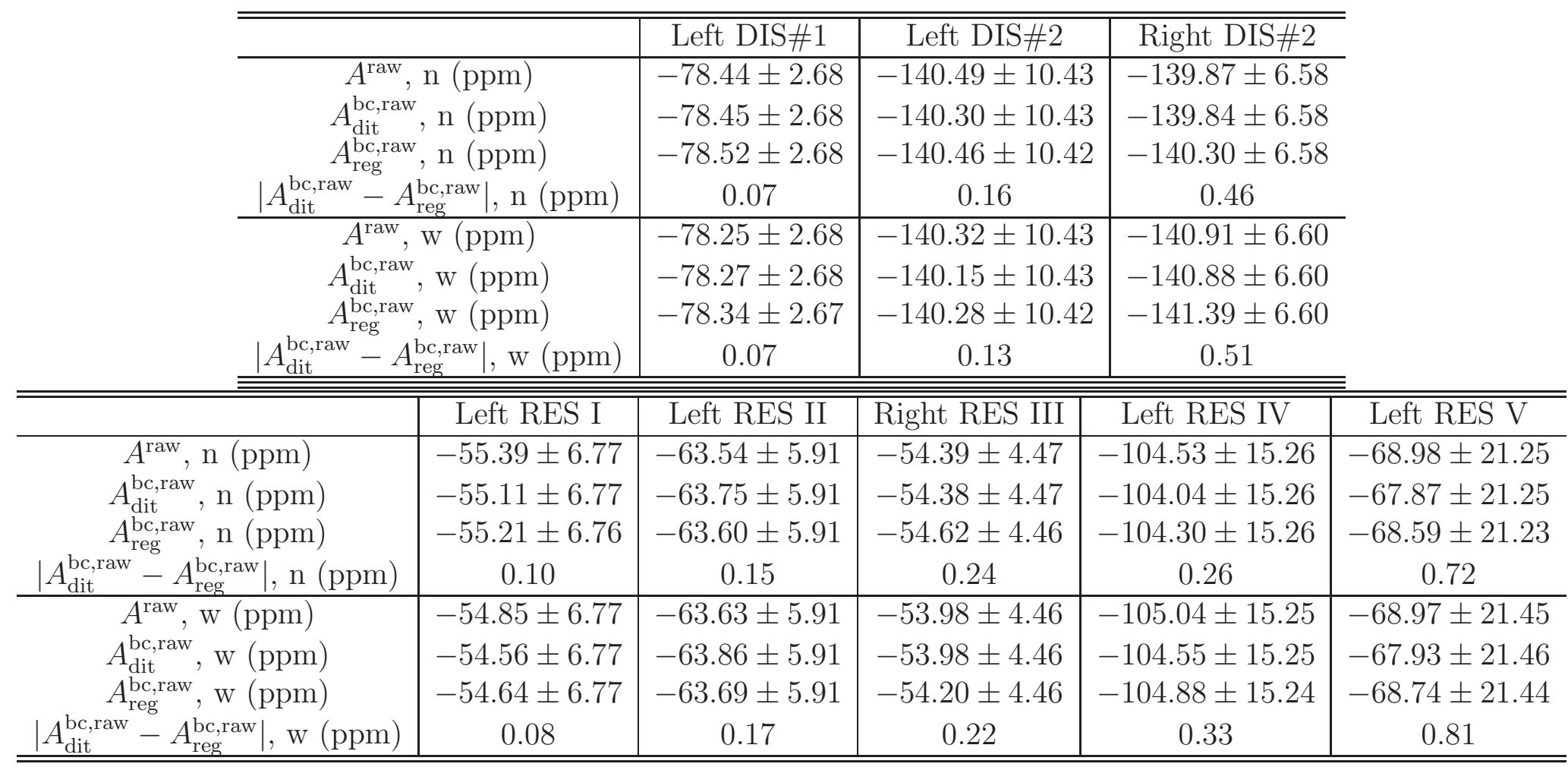

Table 3.3: Corrections to the measured raw asymmetries from the narrow and the wide triggers due to beam energy and position changes using the dithering and the regression methods. The asymmetry errors shown are statistical only. The differences between the two corrected asymmetries, $\left|A_{\mathrm{dit}}^{\mathrm{bc}, \mathrm{waw}}-A_{\mathrm{reg}}^{\mathrm{bc} \text { raw }}\right|$, were used as the uncertainty due to beam corrections. 

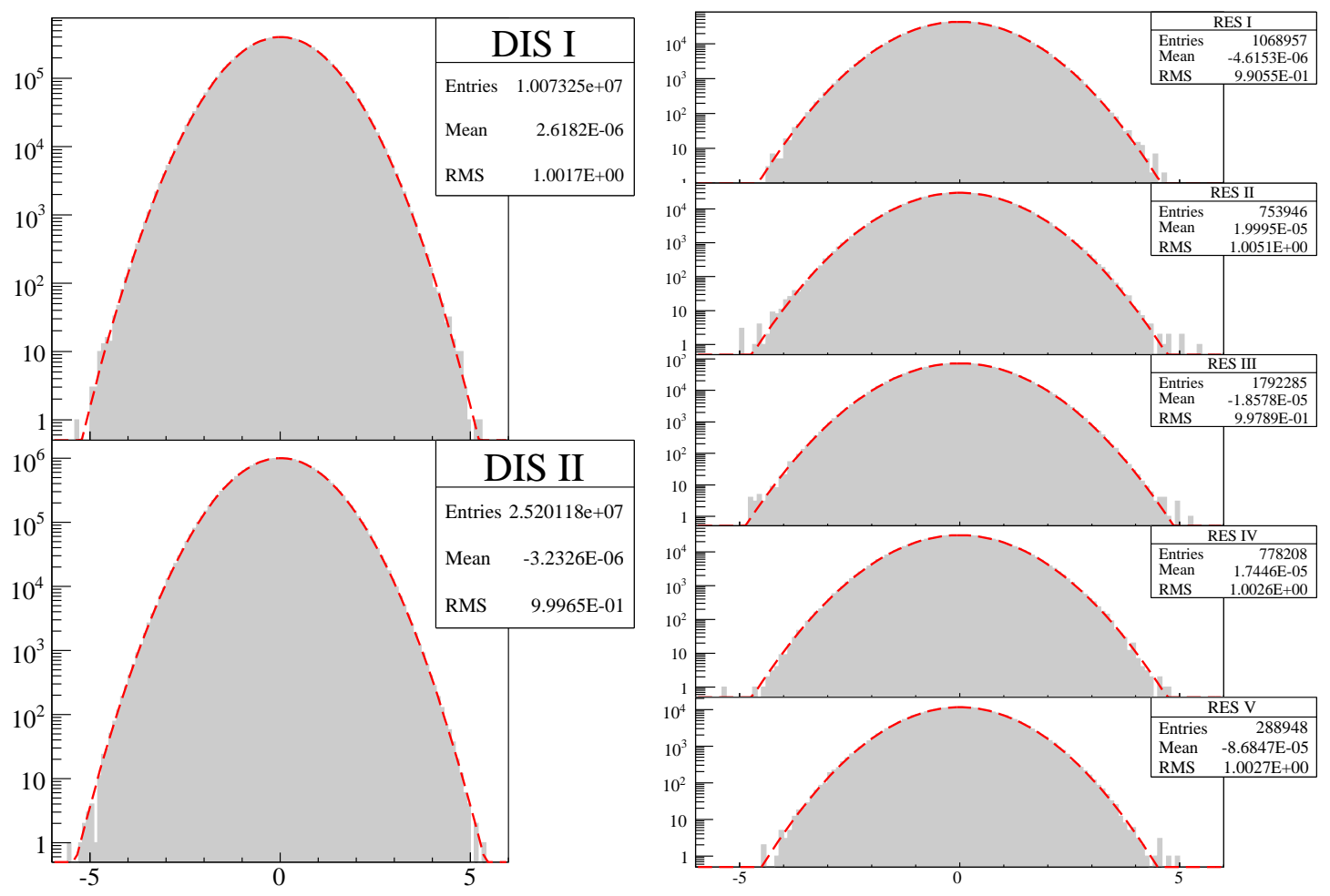

Figure 3.3: Pull plots of the global electron narrow trigger for different kinematics. The shaded areas represent the histograms for the pull $p_{i}$, and the red curves are Gaussian fits to the histograms. 


\subsection{Beam Polarization}

One of the major systematic corrections is the beam polarization, which was applied to the asymmetry as a normalization factor:

$$
A_{\mathrm{phys}}=A_{m} / P_{b}
$$

where $A_{m}$ is the measured asymmetry with beam corrections applied, which is es-

sentially $A_{\text {dit }}^{\text {bc,raw }}, A_{\text {phys }}$ is the physical asymmetry, and $P_{b}$ is the beam's longitudinal polarization.

In Hall $\mathrm{A}$, the beam polarization can be measured independently by the Møller polarimeter and the Compton polarimeter. Setups of the two polarimeters are described in Section 2.3.5. Both polarimeters were used during the experiment and the analysis procedures are presented here, focusing mainly on the Compton analysis.

\subsubsection{Compton Measurements (Photon Detector)}

The upgraded Compton photon detector with an integrating Flash ADC DAQ system is described in detail in Reference $[53,54]$, featuring results from the HAPPEX-III experiment. Running right after HAPPEX-III, the PVDIS experiment inherits the same instrumental setup, thus follows basically the same analysis procedure. However, the much higher background signal induced by the higher beam energy of this experiment casts special challenges to the analysis, especially on the calculation of the analyzing power.

The beam polarization can be extracted from the measured Compton asymmetry $A_{e} x p$ using

$$
P_{b}=\frac{A_{e x p}}{P_{\gamma} A_{t h}}
$$


where $P_{b}$ is the beam polarization, $P_{\gamma}$ is the laser polarization, $A_{\text {exp }}$ is the Compton scattering asymmetry measured by the integrating FADC DAQ and $A_{t h}$ is the theoretical asymmetry, which is also defined as the analyzing power.

\subsubsection{Extraction of $A_{\text {exp }}$}

As described in Ref $[53,54]$, the integrating DAQ has six accumulators implemented, accumulator $0 \sim 5$, representing six different ways of summing the Flash ADC samples. These six accumulators allow for not only asymmetry measurements (Accumulators 0,2 and 4) but also detailed study of backgrounds (Accumulators 1,3 and 5). For this experiment, Accumulator 0 was chosen for the extraction of $A_{\text {exp }}$ based on the consideration that it's insensitive to pedestal drifts of the PMT coupled to the GSO crystal. After deciding which accumulator to use, the proper way of calculating the asymmetry needs to be chosen from the following three:

- Laser-wise: The asymmetry is calculated for each laser cycle with background subtracted locally using the background value averaged over the two adjacent laser-off periods.

- Run-wise: The sum and difference of the signal is calculated for each helicity pair and then averaged over the whole run. The asymmetry is formed by taking the ratio of the two averaged values of opposite helicity states, with the proper subtraction of a mean background value which is also averaged over the whole run.

- Pair-wise: The asymmetry is calculated for each helicity pair, with the subtraction of a run-averaged background.

The run-wise method would be useful if we were running with low beam current, as there wouldn't be enough statistics to perform a laser-wise or pair-wise analysis. 
However, using a run-averaged background subtraction is problematic because the background fluctuates with time. The pair-wise asymmetry distribution becomes non-gaussian when the background is high. Therefore, it's more desirable to use the laser-wise method for extracting $A_{\text {exp }}$, which is what was used for the PVDIS analysis.

\subsubsection{Calculation of $A_{t h}$}

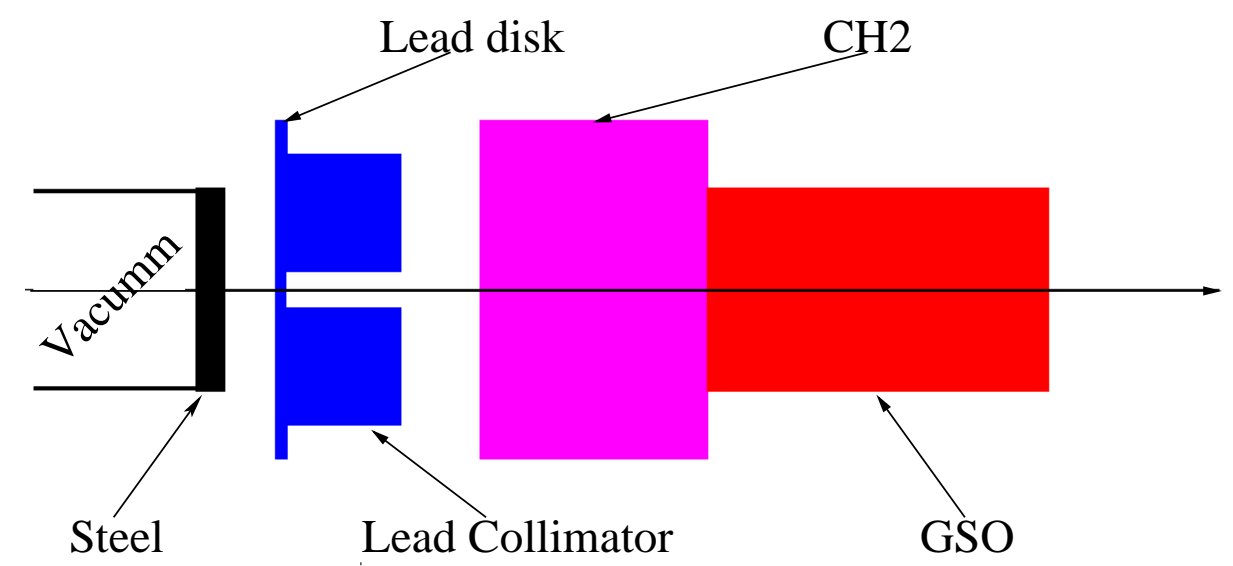

Figure 3.4: Schematic drawing of the background shielding for the Compton photon detector during PVDIS. The drawing is not to scale.

The analyzing power $A_{t h}$ is calculated using a GEANT4 simulation, which takes into account the whole experimental apparatus from right after the Compton scattering point to the DAQ system, including systematic effects induced by the DAQ. The general procedure of the simulation is sketched as the following:

- Scattered photons are generated with a distribution determined from the Compton scattering cross section based on the kinematics. The generated photons spray a geometric and energy phase space large enough to cover the whole acceptance of the photon detector. For each photon event, the Compton asymmetry is also calculated accordingly. 
- The Compton photons then pass through all the materials along the photon beam line and finally reach the GSO photon calorimeter (Figure 3.4). Corresponding radiative energy losses and possible rescattering processes while passing materials are simulated using standard packages of GEANT4.

- Energy deposits of the photons in the calorimeter are then calculated, with a smearing effect added to account for the detector's energy resolution.

- The Energy deposits are then transformed into PMT signal amplitudes, based on a response function determined by an off-line linearity test of the specific PMT used in the experiment. Therefore, the PMT's non-linearity effect is corrected.

- Pileup effects extracted from the data are then added to the PMT amplitudes.

- The analyzing power is calculated by averaging the Compton asymmetries weighted by PMT amplitudes (i.e. energy weighted).

- The histogram of the PMT amplitudes, usually referred to as the "Compton spectrum", can be fitted to the equivalent measured spectrum from data collected by the DAQ. The fitting quality provides an estimation of how well the simulation reproduces the real experiment. Any discrepancy between simulation and data provides a measure of the systematic uncertainty of $A_{t h}$ calculation.

Due to the high electron beam energy of this experiment, the photon detector was flooded by high energy beam-halo bremsstrahlung and low energy synchrotron radiation, which makes the signal-to-background ratio too low for an accurate measurement of the asymmetry. Therefore, as shown in Figure 3.4, a $0.3 \mathrm{~cm}$ radius lead collimator, a $0.2 \mathrm{~cm}$ lead disk, and a $10.2 \mathrm{~cm} \mathrm{CH} 2$ cylindrical were placed in front 


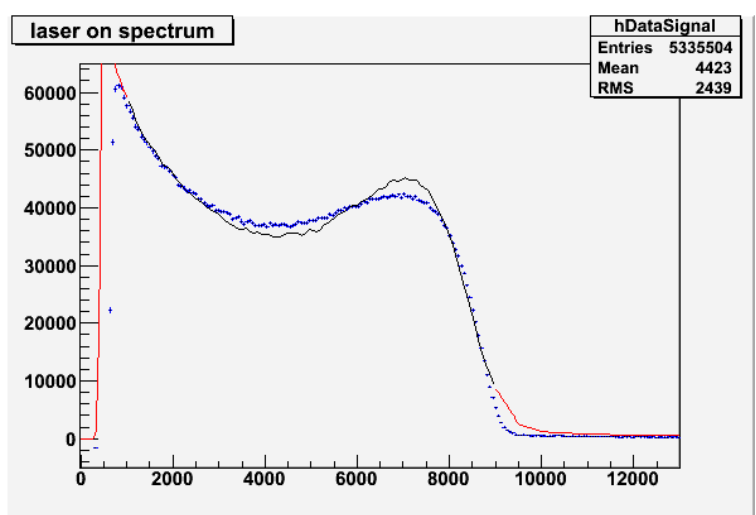

(a) Lead disk thickness $=0.2 \mathrm{~cm}$

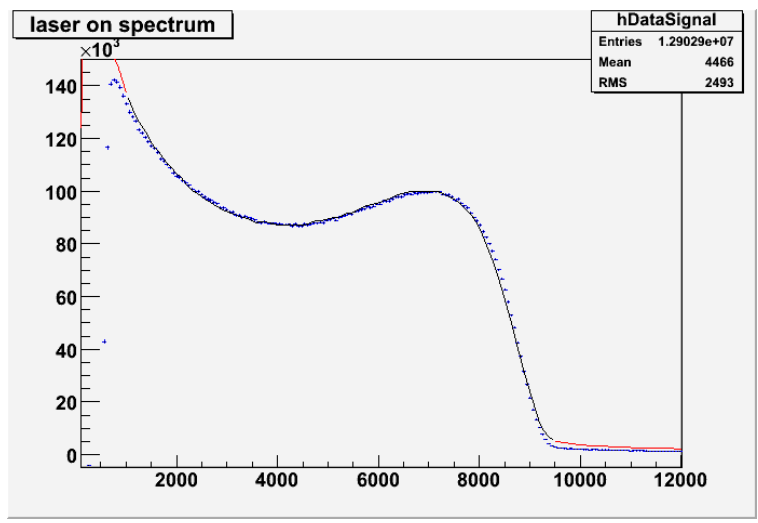

(b) Lead disk thickness $=0.3 \mathrm{~cm}$

Figure 3.5: Compton spectrum.

of the calorimeter for background shielding. These shielding materials can significantly distort the Compton spectrum, and thus add extra systematic uncertainty to the calculation of $A_{t h}$ when they are implemented in the simulation. A lot of effort was taken to fine-tune the Monte Carlo by tweaking input information and parameters such as the smearing effect, the pileup effect, the radius of the collimator, the alignment of the collimator with respect to the photon beam line and the thicknesses of the shielding lead disk and the CH2. It was found that the Compton spectrum is insensitive to most of these tweakings except for the thickness of the lead disk. This is shown in Figure 3.5. Figure 3.5a shows the Compton spectrum generated by simulation (black) fitted to the equivalent spectrum from data (green) for a lead disk of thickness $0.2 \mathrm{~cm}$ (which is the actual thickness and the nominal value used in the simulation). Figure $3.5 \mathrm{~b}$ shows the same result for a lead disk of thickness $0.3 \mathrm{~cm}$. A change of $0.1 \mathrm{~cm}$ in the lead disk's thickness makes the fit almost perfect.

The analyzing power is then calculated using the nominal apparatus setting (the same as Figure 3.5a), and its systematic uncertainty is determined by the difference between the nominal setting and the $0.3 \mathrm{~cm}$ leading disk setting that reproduces the perfect fit (Figure 3.5b). 


\subsubsection{Laser Polarization}

The laser polarization at the Compton interaction point (CIP) was determined by combining the laser polarization at the cavity exit, which was monitored online, and a transfer function obtained from off-line studies of the laser system. The transfer function relates the laser polarization at the CIP to that measured at the cavity exit. The laser polarization was observed to be $\sim 99 \%$ during PVDIS. The systematic uncertainty of the laser polarization comes mainly from the transfer function, which is $0.80 \%$ relative.

\subsubsection{Compton Results}

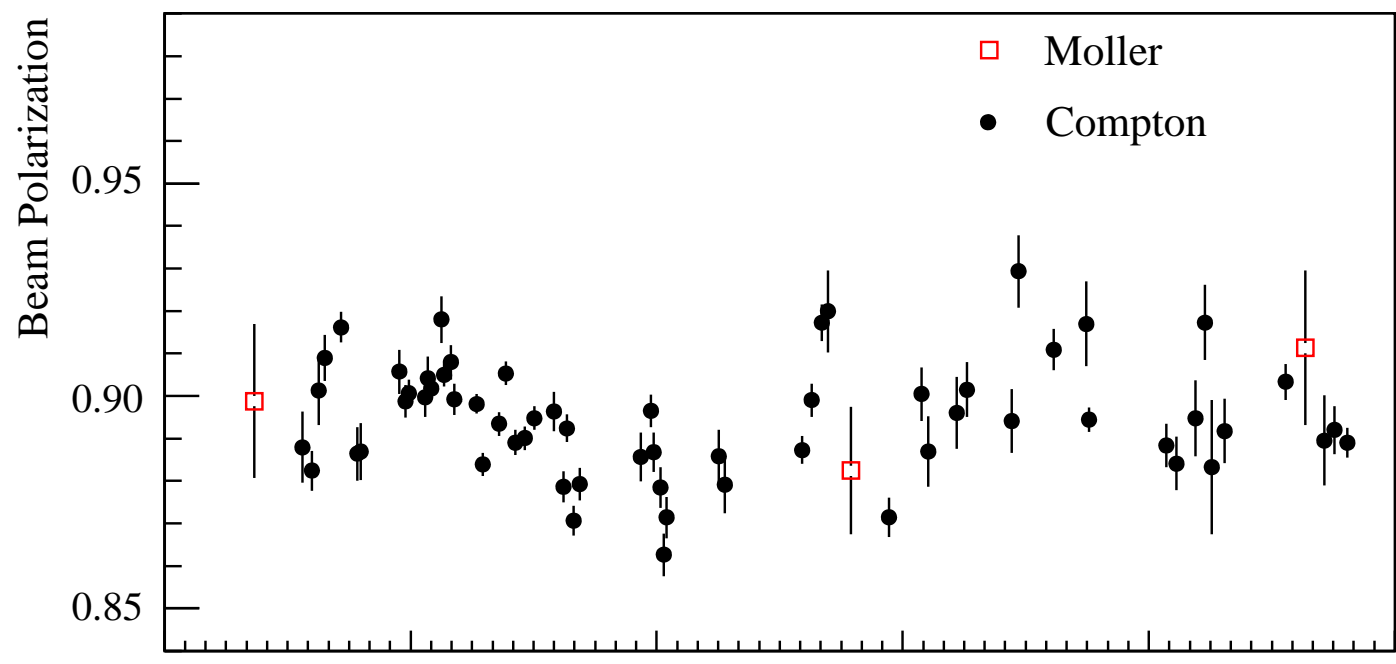

Dec.01,2009 Dec.04,2009 Dec.07,2009 Dec.11,2009 Dec.14,2009 Dec.17,2009

Date

Figure 3.6: Beam polarization history from the Compton polarimeter (black solid circles), plotted with the Møller results (red open squares) available at the same time period. The error bars for Compton are statistical only, while for Møller they include systematic uncertainties. A constant fit to Compton measurements gave an average of $89.45 \%$ while the average of Møller results was $88.74 \%$.

The Compton polarimeter initially suffered from a high background and only produced results in the last three weeks of the 1.5-month $6 \mathrm{GeV}$ run period. The 
Compton polarimeter was also not available during the $4.8 \mathrm{GeV}$ run period. Figure 3.6 shows the beam polarization results from Compton during its functioning time period, together with Møller results at the same time for comparison.

\subsubsection{Møller Measurements}

Møller measurements were taken routinely every several days, and opportunistically whenever there were major changes on the experiment setup such as beam energy change and kinematics change. Figure 3.7 shows the Møller results over the whole running period. The systematic uncertainty of these measurements are $1.7 \% \sim 2.0 \%$ (relative) and the statistical uncertainties are on the order of $0.1 \%$. Details of the uncertainty evaluation are summarized in Ref [64].

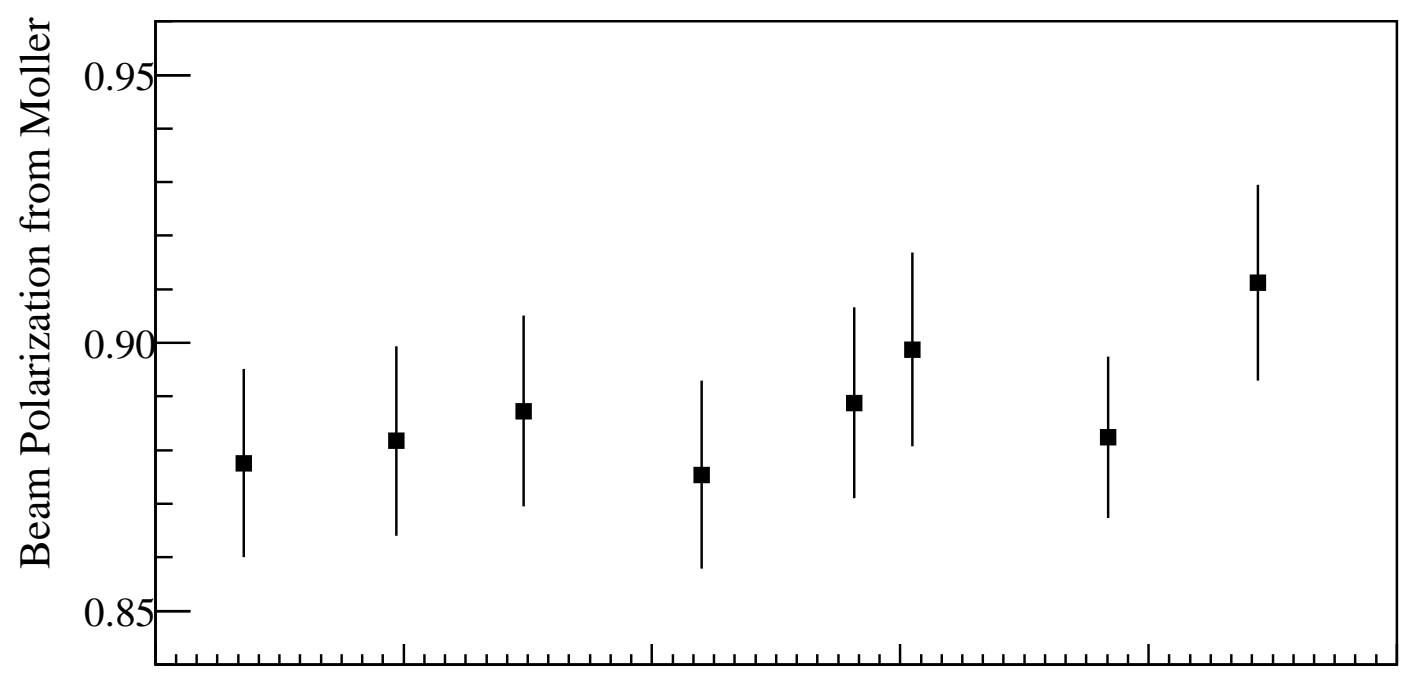

Nov.02,2009 Nov.12,2009 Nov.22,2009 Dec.02,2009 Dec.11,2009 Dec.21,2009

Date

Figure 3.7: Polarization history from the Møller polarimeter measurements taken with a beam energy of $6.067 \mathrm{GeV}$. The error bars include systematic error. An additional measurement was done with a beam energy of $4.867 \mathrm{GeV}$ at the end of the run period, which gave similar polarization but is not shown here. 


\subsubsection{Combining Compton and Møller}

The experimental asymmetries were corrected for the beam polarization as follows:

1. When there was no Compton measurement (before Dec. 2nd and after Dec. 17th, 2009), only Møller results were used. Each Møller measurement result was used for the consecutive days until the next measurement was available.

2. When there were both Compton and Møller measurements (from Dec. 2nd to Dec. 17th, 2009), the Compton data were averaged first for the time interval between two Møller measurements, then was averaged with the corresponding Møller measurement from the beginning of the interval. The systematic uncertainties of the two polarimeter were combined. Each combined result was used for the consecutive days following a Møller measurement, until the next Møller measurement was available.

3. The beam polarization was corrected run by run for DIS\#1 and \#2. For resonance kinematics, the run period was short and a single correction was made for all runs of the same kinematics.

The beam polarization corrections are shown in Table 3.4 for all kinematics.

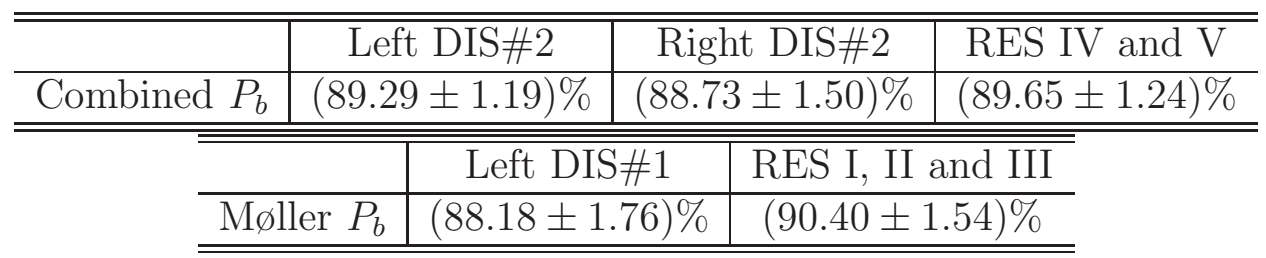

Table 3.4: Average beam polarization $P_{b}$ for each kinematics. These are either the combined results of Compton and Møller measurements, or results from Møller alone, depending on which polarimeter was available during the corresponding run period. For DIS\#1 and \#2 the corrections were applied run-by-run and the statisticallyaveraged value of $P_{b}$ is shown. For resonance kinematics, each setting had short running period and a single value was used for all runs of each period. 


\subsection{Deadtime Correction}

Since we used a counting DAQ, deadtime is an inevitable systematic effect. Right after an event triggers the DAQ system, the system remains unresponsive ("dead") to another event for a certain amount of time $\tau$. This time $\tau$ is called the deadtime of the system. For physics events occurring at a rate $R$, the probability of an event falling in the time window $\tau$ of an preceding event, thus getting lost due to the deadtime, is $1-e^{-R \tau}$. Therefore the measured rate $R_{m}$ is

$$
R_{m}=R\left(1-\left(1-e^{-R \tau}\right)\right)=R e^{-R \tau}
$$

In the limit of $R \tau \ll 1$, which is true for this experiment as we will see, Equation 3.7.1 can be approximated by:

$$
R_{m} \simeq R(1-R \tau)
$$

This implies two parallel equations for different helicity states:

$$
R_{m}^{ \pm}=R^{ \pm}\left(1-R^{ \pm} \tau\right)
$$

The fact that the deadtime correction factor $R \tau$ depends on rate is essential, since it results in different correction factors for different helicity states. Therefore, when we form the asymmetry by taking the ratio of rates, this factor does not cancel out:

$$
A_{m}=\frac{R_{m}^{+}-R_{m}^{-}}{R_{m}^{+}+R_{m}^{-}} \simeq A(1-R \tau)
$$

where $A_{m}$ and $A$ are the measured and the physical asymmetries respectively. As we can see, deadtime goes into the asymmetry directly as a first order correction, so it is an important systematic effect and needs to be understood thoroughly. 
In order to evaluate the deadtime of the fast-counting DAQ system, we decompose the total deadtime into contributions from different parts of the DAQ. Specific techniques were applied to study these contributions in detail. At the same time, a full-scale simulation was developed to simulate the DAQ as a whole. Comparisons between the simulation and the data obtained from detailed study on the composing parts provides justifications of the simulation. The simulation then gives the final results of the total deadtime.

\subsubsection{Decomposition of the Deadtime}

A full description about the electronics and the trigger logic of the PVDIS DAQ is presented in Section 2.6.2. The total deadtime can be broken down into three contributing parts:

- The "group" deadtime: For each individual group, the preshower and shower PMT signals suffer a deadtime when passing through the discriminators and forming the coincidence group trigger at the logic AND modules.

- The "VETO" deadtime: The signal formed by combining the Scintillator and Cerenkov signals serves as a "GATE" signal opened to accept group triggers. This signal was fed to the VETO input of logic AND (logic OR) modules for forming the group electron (pion) triggers, and was therefore referred to as the "VETO" signal. In the following we will use VETO and GATE interchangeably. Any loss of the VETO signal due to the deadtime of the "VETO circuit" would cause a failure in forming the group trigger.

- The "OR" deadtime: All group triggers are 'OR'ed together at the end to form the global trigger. The logic OR module is unable to distinguish triggers from 
different groups if they happen one after another within a time window that equals the signal width of the group trigger itself.

These three components of the deadtime are basically independent of each other and don't interfere to the first order, thus the total deadtime is a direct sum of all three. However, the system is rather complex and the most reliable way to get the total deadtime is by a software simulation.

\subsubsection{Methods to study the deadtime}

\subsubsection{Group Deadtime: The Tagger Method}

In order to study the group deadtime, a "tagger", which is a fixed frequency logical pulser signal generated using gate generators, was mixed with real physics signals (preshower and shower PMT signals) and fed to the DAQ. The tagger passed through the exact same set of electronics as physics signals do, thus should "see" the same deadtime. This tagger scheme was applied to every individual group. At the end of each group, we pick out the tagger from the output trigger signals by requiring a coincidence, using a logic AND module, between the group electron trigger and the delayed tagger. The delayed tagger is a copy of the original tagger but delayed by the amount of the DAQ's response time. If there is no physics signal, since the time spacing between tagger pulses is a constant and much larger than any electronics width and the total response time of the DAQ, the tagger passes through the DAQ deadtimelessly. In this case, every tagger will produce an electron trigger and then a coincidence signal, thus the output coincidence rate $R_{c}$ will be exactly the same as the input tagger rate $R_{i}$. However, with the presence of high-rate physics signals, distributed randomly with respect to tagger pulses, a fraction of the tagger pulses will be killed by their closely preceding physics signals due to deadtime, which will 
result in a smaller $R_{c}$ than $R_{i}$. The difference between $R_{c}$ and $R_{i}$ provides a direct measurement of the group deadtime.

One effect that complicates this tagger method is the so-called pileup effect, as explained in Figure 3.8. A pileup happens when the preceding physics signal happens very close to the tagger, so that although the tagger is killed, the physics signal itself produces a trigger which falls within the delayed tagger's "coincidence range" (which spans a width of $w+t_{1}$, see the later two scenarios of $\mathrm{C}$ in Figure 3.8). In this case, a coincidence is still formed despite the loss of the tagger signal due to deadtime. We should therefore subtract such false coincidence from the deadtime calculation.

The pileup effect can be investigated by studying the timing spectrum of the coincidence signal using fbTDCs. Bottom plot of Figure 3.8 shows a typical fbTDC pileup spectrum, where the integrands $I_{0}, I_{1}$ and $I_{2}$ correspond to the three different cases of scenario $\mathrm{C}$ in the upper plot respectively. Theoretically, the relative fractions of $I_{1}$ and $I_{2}$ with respect to $I_{0}$ depend on the physics signal rate $R_{p}$, and the timing setting constants $w$ and $t_{1}$, specifically, $I_{1} / I_{0}=R_{p} t_{1}$ and $I_{2} / I_{0}=R_{p} w$. These relations were checked and found to agree very well with data.

From the fbTDC pileup spectrum, we extract the pileup correction factor:

$$
p=\left(I_{1}+I_{2}\right) / I_{0}
$$

The group deadtime was calculated as:

$$
D T_{\text {group }}=\frac{R_{i}-(1-p) R_{c}}{R_{i}} \simeq \frac{R_{i}-R_{c}}{R_{i}}+p
$$

We will call the first term on the right hand side "count loss" to distinguish from the pileup factor $p$. For each group, the count loss and the pileup correction were measured separately using scalers and fbTDCs, then the two were added together to 


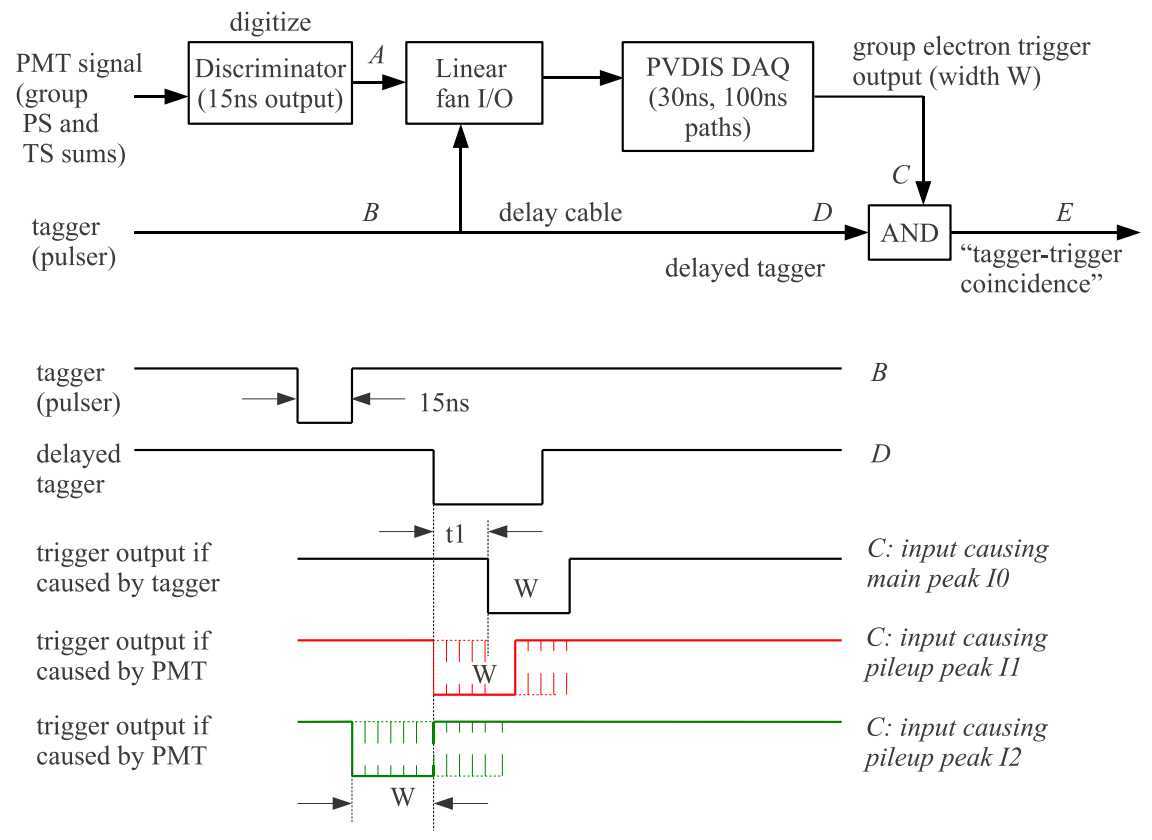

from signals triggered by tagger itself

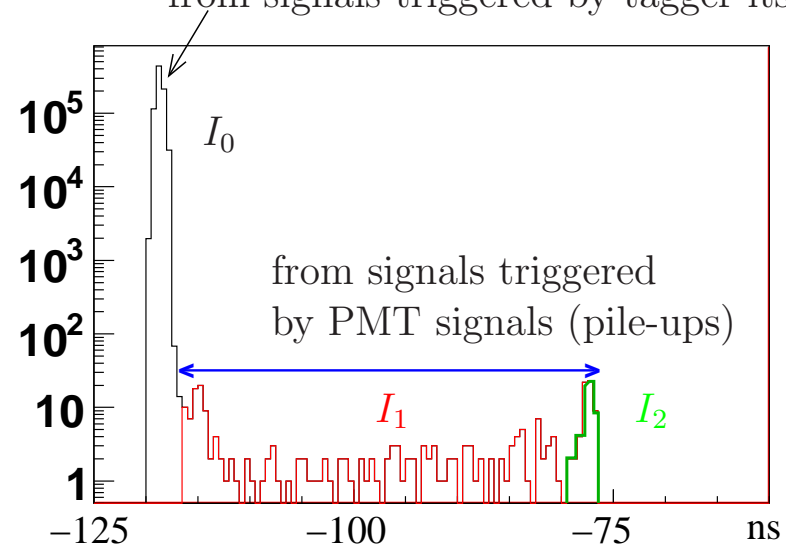

Figure 3.8: Top: schematic diagram for the tagger setup and signal timing sequence. Bottom: fbTDC spectrum for the relative timing between tagger-trigger coincidence and the input tagger, in 0.5-ns bins. The fbTDC module works in the multi-hit mode. Two different scenarios are shown: 1) Main peak $I_{0}$ : when there is no PMT signal preceding the tagger, the tagger triggers the DAQ and forms a tagger-trigger coincidence. 2) Pileup events $I_{1}$ and $I_{2}$ : when there is a PMT signal preceding the tagger by a time interval shorter than the delayed tagger width, the PMT signal triggers the DAQ and forms a tagger-trigger coincidence signal with the delayed tagger. 


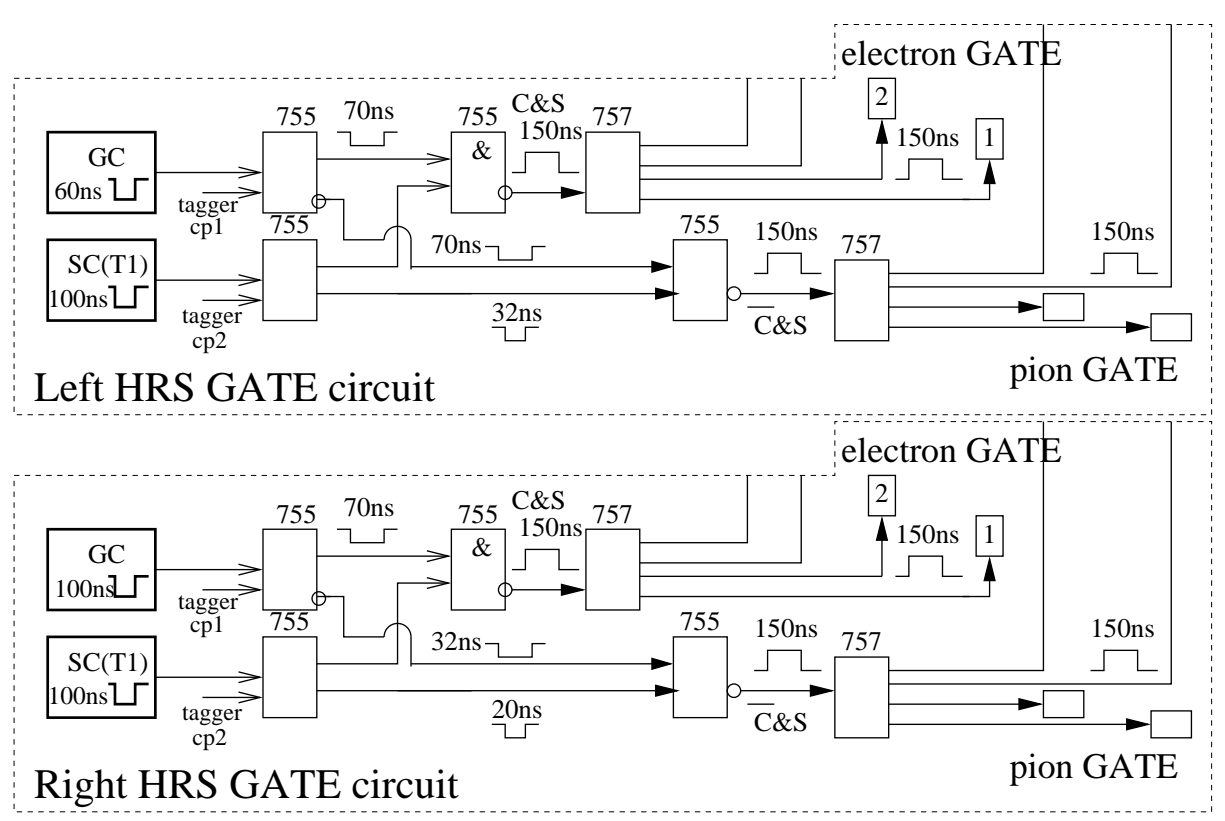

Figure 3.9: The VETO circuits of the Parity DAQ for Left (top) and Right (bottom) spectrometers.

form the total group deadtime.

\subsubsection{VETO Deadtime: Flash ADC Method}

Deadtime rises from the VETO circuit(Figure 3.9) as a result of the fact that the two input signals, the Cerenkov (GC) and the Scintillator (SC) signals, were not purely triggered by electrons. The intrinsic deadtime of the SC and GC also contribute. A typical electron triggers both the Cerenkov and the Scintillator at the same time, which produces a VETO signal by taking the coincidence of the two. However, apart from electrons, the $\mathrm{SC}$ can also be triggered by pions. In addition, both the GC and the SC signals have significant portions caused by electronics noises. These nonelectron-triggered signals were randomly distributed in time, so sometimes they may pile up with the electron-triggered signals. The pileup of a non-electron-triggered signal and an electron-triggered signal for one of the two inputs will cause a misalignment in time between the $\mathrm{GC}$ and the $\mathrm{SC}$ signals. This timing misalignment, together 
with the specific output signal width settings of the Phillips-755 modules used in the circuit, may cause the two signals to miss each other when forming the coincidence to produce the VETO signal, causing a VETO deadtime.

Based on the considerations above, the VETO deadtime, or specifically, the probability of the VETO circuit failing to generate a VETO signal for a real electron, should be proportional to the non-electron-triggered signal rates as well as the signals' widths. The theoretical formula of the VETO deadtime (represented as a fractional loss) is:

$$
D T_{V E T O}=R_{S C . n o . G C}\left(W_{S C . \text { in }}-W_{S C . \text { out }}\right)+R_{G C . n o . S C}\left(W_{G C . \text { in }}-W_{G C . o u t}\right),
$$

where the $R_{S C . n o . G C}$ and $R_{G C . n o . S C}$ refer to the non-electron-triggered rates of the Scintillator and Cerenkov detectors respectively, and the $W_{\text {in/out }}$ refer to the input/output signal width of the Phillips-755 module. Note that if the electronics used to generate the Scintillator and the Cerenkov signals have intrinsic deadtimes that are longer than $W_{S C . i n}$ and $W_{G C . i n}$, these intrinsic deadtimes should be used in place of the measured $W_{S C . i n}$ and $W_{G C . i n}$.

The direct observation of VETO deadtime comes from the Flash ADC (FADC) data, which we took during the experiment with the FADCs turned on. Figure 3.10 shows the snapshots from a sample FADC event that represents and contributes to the VETO deadtime. The ratio of such events to the total number of events is the VETO deadtime.

\subsubsection{OR Deadtime: Analytical Analysis}

The OR deadtime from the final logical OR module is quite straightforward. When two electron triggers from different groups overlap in time as they go into the logical 

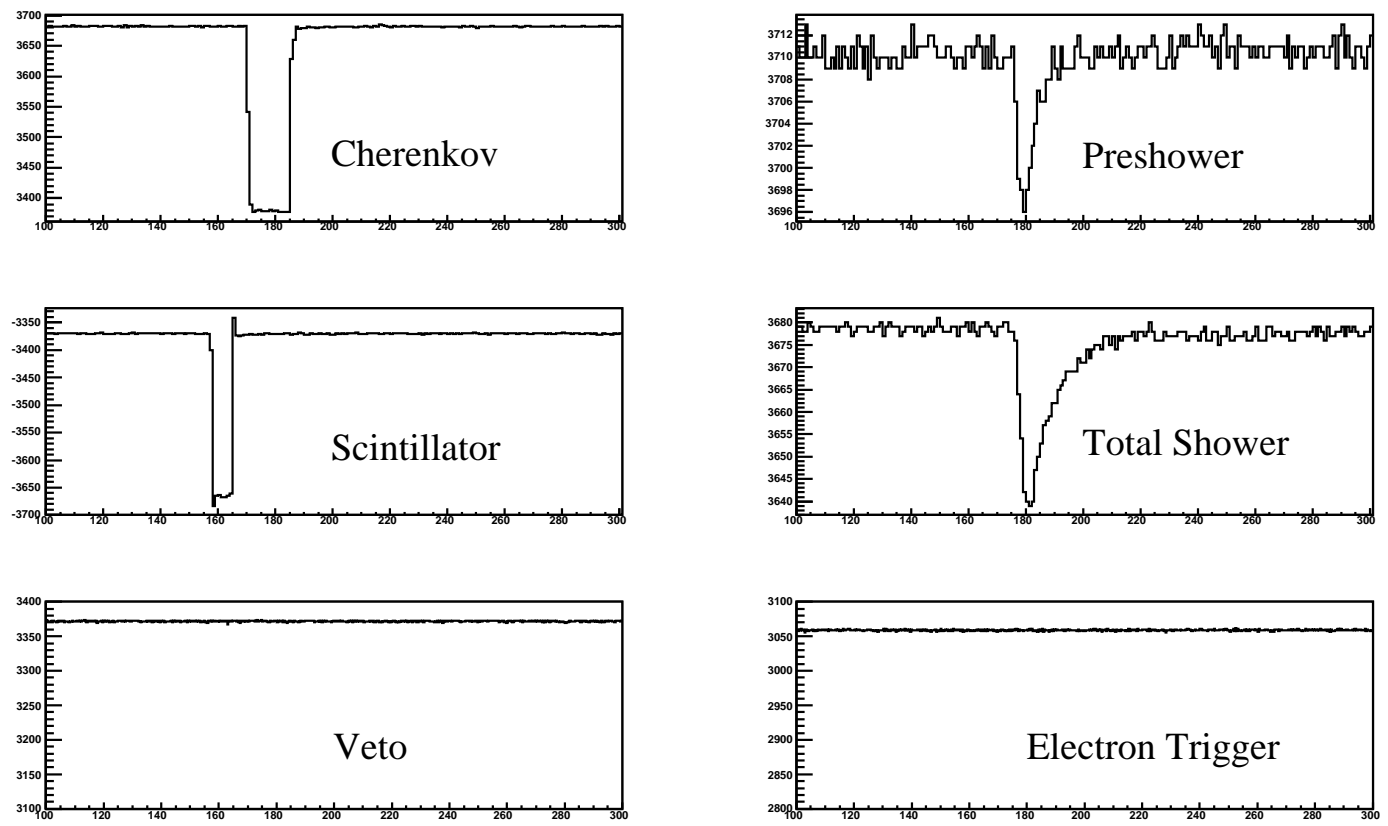

Figure 3.10: FADC snapshots of one example event that suffered VETO deadtime. The event triggered Cerenkov, Scintillator and the Preshower and Shower, meaning it was a good electron. However, the DAQ fails to generate the VETO signal due to the VETO deadtime, which results in the loss of the electron trigger. The early timing of the Scintillator signal indicates that it was from a non-electron-triggered signal that preceded the electron. 
OR module, they will only be recognized and recorded as one. An analytical analysis was performed and the OR deadtime can be directly calculated knowing the trigger rates and trigger signal widths of each individual group.

\subsubsection{Software Simulation}

A full-scale software simulation was developed to study the DAQ deadtime and provided the final deadtime correction to the asymmetry. The simulation takes as inputs the physical event rates and the PMT analog signal shapes, obtained from FADC snapshots. It then simulates the response of the whole DAQ assembly, including all standard electronic modules, how they connect to each other, and more importantly the timing sequences among them. In other words, we managed to rebuild the PVDIS DAQ on a software level. The advantage of a software simulation is that in principle we can simulate everything happening anywhere in the DAQ system at any particular time, while we don't have such luxury with the data taken during the experiment. From data we can only study part of the deadtime, while using the simulation we can easily calculate the total deadtime by comparing the final simulated output event rate with the initial input event rate. However, before we use the simulation to obtain the total deadtime of the DAQ, we need to confirm the accuracy of the simulation. This was achieved by comparing the simulation with data on different deadtime components.

\subsubsection{DATA-Simulation Comparison}

\section{Group Deadtime}

Figures 3.11 and 3.12 show direct comparisons of the group deadtime between tagger data and the simulation, for one central group on each HRS. The simulation ran up to higher event rates in order to acquire more statistics with a reasonable 


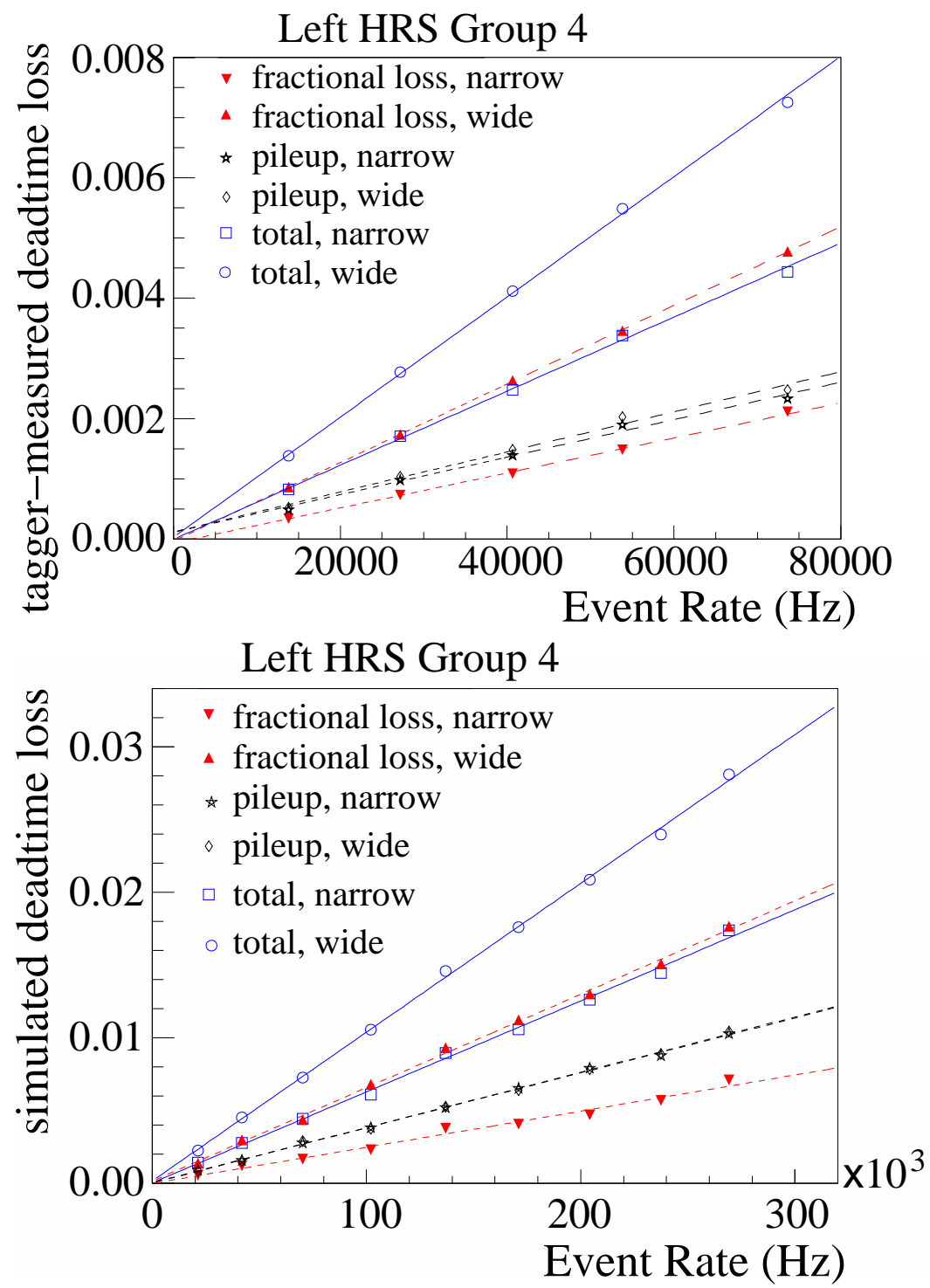

Figure 3.11: Deadtime loss in percent vs. event rate from the tagger method for group 4 on the Left HRS. Top: actual deadtime loss from tagger measurements; Bottom: simulated deadtime loss of the tagger. The tagger fractional count loss $1-$ $R_{o} / R_{i}$ (red) and the pileup correction $p$ (black) are combined to form the total group deadtime $D$ (blue). These data were taken (or simulated) at a $Q^{2}$ of $1.1(\mathrm{GeV} / \mathrm{c})^{2}$. To minimize the statistical uncertainty while keeping the computing time reasonable, the simulation used higher event rates than the tagger measurement. The total group deadtime can be determined from the linear fit slope coefficients: tagger data narrow $p_{1}=(61.5 \pm 0.2) \times 10^{-9} \mathrm{~s}$, wide $p_{1}=(99.9 \pm 0.3) \times 10^{-9} \mathrm{~s}$, simulation narrow $p_{1}=(62.5 \pm 1.4) \times 10^{-9} \mathrm{~s}$, wide $p_{1}=(102 \pm 1.3) \times 10^{-9} \mathrm{~s}$. Group 4 is from the central blocks of the lead-glass detector and has the highest rate among all groups. 

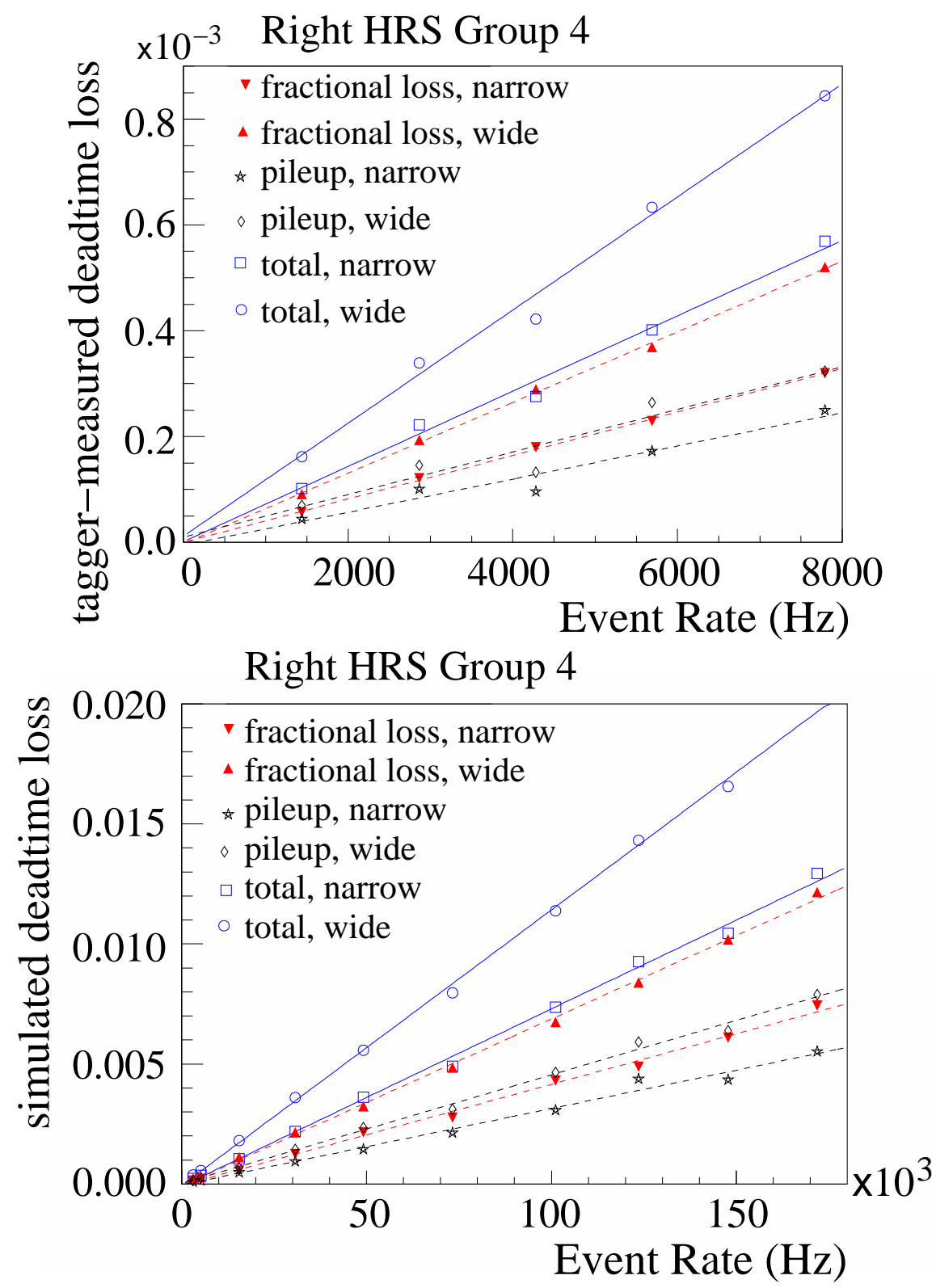

Figure 3.12: Deadtime loss in percent vs. event rate from the tagger method for group 4 on the Right HRS. Top: tagger data; Bottom: simulation. These data were taken (or simulated) at a $Q^{2}$ of $1.9(\mathrm{GeV} / c)^{2}$. The total group deadtime can be determined from the linear fit slope coefficients: tagger data narrow $p_{1}=(71.1 \pm 0.9) \times 10^{-9} \mathrm{~s}$, wide $p_{1}=(107 \pm 1.2) \times 10^{-9} \mathrm{~s}$, simulation narrow $p_{1}=(73.9 \pm 1.5) \times 10^{-9} \mathrm{~s}$, wide $p_{1}=(115 \pm 1.5) \times 10^{-9} \mathrm{~s}$. Group 4 is from the central blocks of the lead-glass detector and has the highest rate among all groups. See Fig. 3.11 caption for details. 
computing time. The simulated group deadtime was also broken down into pileup and count loss. As we can see, the results from tagger data agree well with the simulation. They both show qualitatively larger deadtime of the wide path than the narrow path. Quantitatively, the slope of a linear fit to such deadtime loss v.s. event rate plot gives the deadtime in seconds. For the wide path, a $\sim 100 \mathrm{~ns}$ of deadtime is observed, which is precisely the discriminators' output widths as expected. For the narrow path, although the discriminators' widths were set to be 30ns, the deadtime was expected to be dominated by the intrinsic widths of the analog PMT signals, which were on the level of $60 \sim 70$ ns according to the FADC snapshots. Both the tagger data and the simulation again agree with this expectation.

\section{VETO Deadtime}

All the FADC channels on each HRS share the same common stop signal, which makes it possible to study the relative timing sequences among all the channels. By setting the proper software thresholds on the FADC snapshots, we can transform these snapshots, either of logical or of analog signals, into TDC spectrums. Such TDC spectrums provide us a direct visualization of the VETO deadtime: a "dead zone" on the spectrum, as shown in Figure 3.13. Also, numerical deadtime results can be extracted from the same TDC spectrums by taking the ratio of the dead zone area $\left(N_{1}\right)$ and the area of the main peak near $0 \mathrm{~ns}\left(N_{0}\right)$. The extracted deadtime results were compared with the simulation results. Figure 3.14 shows the agreement between the results from FADC data and the simulation, as well as the theoretical calculations.

\section{OR Deadtime}

The OR deadtime from simulation is calculated by subtracting the group deadtime and the VETO deadtime from the total deadtime, all three of which can be directly extracted from the simulation. The calculated OR deadtime from simulation 

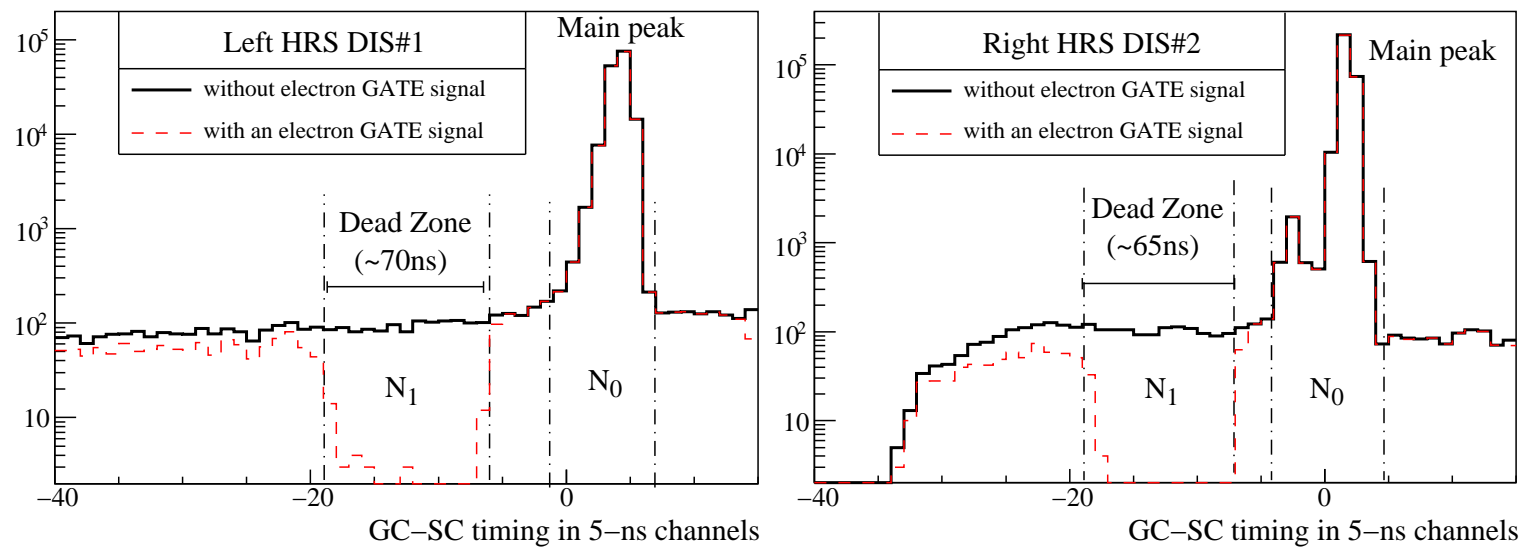

Figure 3.13: Timing difference between Scintillator and Cerenkov signals in 5-ns channels extracted from FADC data. The fractional loss of electron events due to VETO deadtime can be estimated using the ratio of $N_{1} / N_{0}$, where $N_{1}$ is the count difference between the two spectra in the dead zone, and $N_{0}$ is the counts under the main peak near $0 \mathrm{~ns}$.
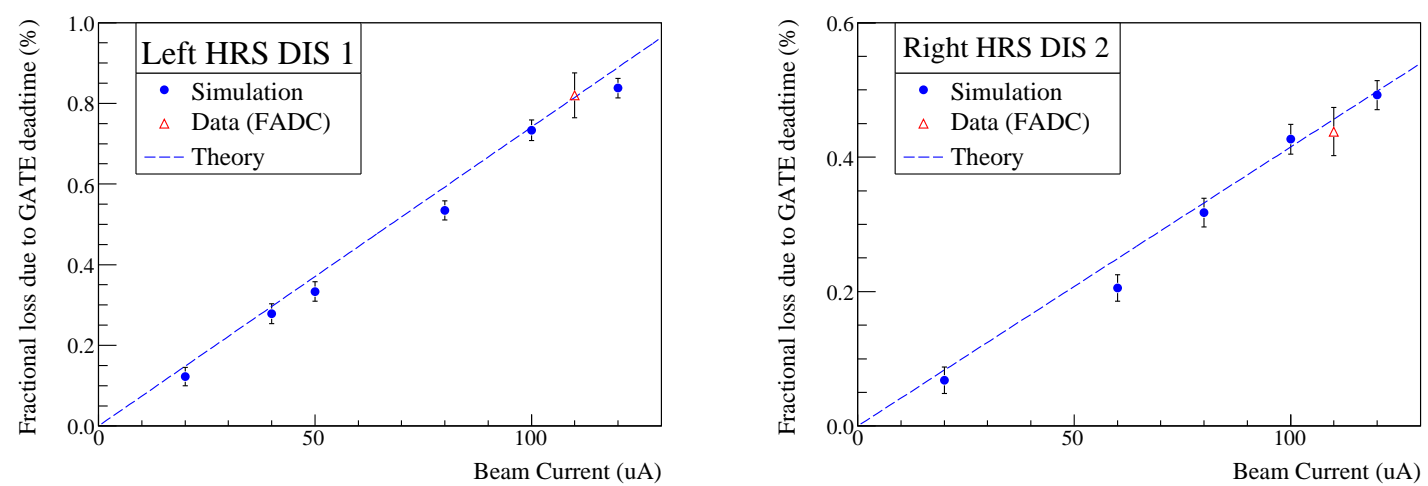

Figure 3.14: VETO deadtime for both HRS from FADC analysis. Shown here is the comparison among simulation, data and theoretical calculation using Equation 3.7.7. 
is compared with the results from an analytic analysis. The difference between the analytic analysis and the simulation is used as the systematic uncertainty of the OR deadtime The OR deadtime only contributes a small fraction to the total deadtime.

\subsubsection{Deadtime Results}

Being confirmed by data, the simulation is now justified to provide the total deadtime for our DAQ system. The deadtime results for different kinematics are shown in Figure 3.15, plotted versus beam current. And Table 3.5 shows the break down of the three contributions, together with their systematic errors. The relation between deadtime and beam current is fitted using first order polynomial, the fitting parameters of which were then used to calculate the deadtime for each run at the specific beam current of that run. This way, a complete run-by-run deadtime correction was available and applied to the whole data set.

\subsection{Particle Identification}

The separation of electrons and pions at the hardware level is achieved by the gas Cerenkov detector and a double-layered lead glass counter (Section 2.5.2 and 2.6.2). The particle identification (PID) efficiencies of these two detectors are usually characterized by two variables: the electron detection efficiency $\eta_{e}$ and the pion rejection factor $\eta_{\pi}$, which are defined as:

$$
\eta_{e}=\frac{\text { number of electrons identified }}{\text { total number of electrons entering the detector }}
$$




\begin{tabular}{c|c|c|c|c|c}
\hline \hline \multirow{2}{*}{ Kinematics } & \multirow{2}{*}{ Path } & \multicolumn{3}{|c|}{ fractional contribution } & Total deadtime \\
& & Group & VETO & OR & loss at $100 \mu \mathrm{A}$ \\
\hline \multirow{2}{*}{ DIS\#1,Left HRS } & $\mathrm{n}$ & $(20.6 \pm 2.1) \%$ & $(51.3 \pm 3.5) \%$ & $(28.1 \pm 4.7) \%$ & $(1.45 \pm 0.09) \%$ \\
& $\mathrm{w}$ & $(29.5 \pm 2.4) \%$ & $(45.3 \pm 3.1) \%$ & $(25.3 \pm 4.6) \%$ & $(1.64 \pm 0.10) \%$ \\
\hline \multirow{2}{*}{ DIS\#2,Left HRS } & $\mathrm{n}$ & $(5.4 \pm 0.8) \%$ & $(81.1 \pm 5.5) \%$ & $(13.5 \pm 7.0) \%$ & $(0.50 \pm 0.04) \%$ \\
& $\mathrm{w}$ & $(8.4 \pm 0.4) \%$ & $(77.3 \pm 5.3) \%$ & $(14.3 \pm 8.0) \%$ & $(0.52 \pm 0.05) \%$ \\
\hline \multirow{2}{*}{ DIS\#2,Right HRS } & $\mathrm{n}$ & $(4.6 \pm 0.4) \%$ & $(72.9 \pm 6.0) \%$ & $(22.6 \pm 17.4) \%$ & $(0.57 \pm 0.10) \%$ \\
& $\mathrm{w}$ & $(6.9 \pm 0.7) \%$ & $(71.0 \pm 5.8) \%$ & $(22.1 \pm 17.9) \%$ & $(0.58 \pm 0.11) \%$ \\
\hline \multirow{2}{*}{ RES I, Left HRS } & $\mathrm{n}$ & $(26.3 \pm 3.8) \%$ & $(39.3 \pm 2.7) \%$ & $(34.4 \pm 1.8) \%$ & $(1.45 \pm 0.07) \%$ \\
& $\mathrm{w}$ & $(37.2 \pm 2.1) \%$ & $(34.3 \pm 2.3) \%$ & $(28.5 \pm 3.1) \%$ & $(1.66 \pm 0.07) \%$ \\
\hline \multirow{2}{*}{ RES II, Left HRS } & $\mathrm{n}$ & $(27.6 \pm 4.3) \%$ & $(38.8 \pm 2.7) \%$ & $(33.6 \pm 7.5) \%$ & $(2.19 \pm 0.20) \%$ \\
& $\mathrm{w}$ & $(38.3 \pm 1.9) \%$ & $(33.2 \pm 2.3) \%$ & $(28.5 \pm 7.0) \%$ & $(2.56 \pm 0.19) \%$ \\
\hline \multirow{2}{*}{ RES III, Right HRS } & $\mathrm{n}$ & $(22.9 \pm 1.8) \%$ & $(60.0 \pm 4.9) \%$ & $(17.1 \pm 18.5) \%$ & $(1.96 \pm 0.38) \%$ \\
& $\mathrm{w}$ & $(30.8 \pm 3.1) \%$ & $(51.8 \pm 4.3) \%$ & $(17.4 \pm 12.7) \%$ & $(2.27 \pm 0.31) \%$ \\
\hline \multirow{2}{*}{ RES IV, Left HRS } & $\mathrm{n}$ & $(14.5 \pm 1.9) \%$ & $(63.7 \pm 4.4) \%$ & $(21.9 \pm 3.0) \%$ & $(0.75 \pm 0.04) \%$ \\
& $\mathrm{w}$ & $(21.5 \pm 1.0) \%$ & $(58.2 \pm 4.0) \%$ & $(20.3 \pm 2.9) \%$ & $(0.82 \pm 0.04) \%$ \\
\hline \multirow{2}{*}{ RES V, Left HRS } & $\mathrm{n}$ & $(15.5 \pm 2.1) \%$ & $(68.3 \pm 4.7) \%$ & $(16.2 \pm 5.7) \%$ & $(1.03 \pm 0.08) \%$ \\
& $\mathrm{w}$ & $(22.7 \pm 1.1) \%$ & $(61.7 \pm 4.2) \%$ & $(15.6 \pm 3.0) \%$ & $(1.14 \pm 0.06) \%$ \\
\hline \hline
\end{tabular}

Table 3.5: Simulated DAQ deadtime loss for all kinematics and for both narrow (n) and wide (w) paths, along with the fractional contributions from Group, VETO and OR deadtimes. 

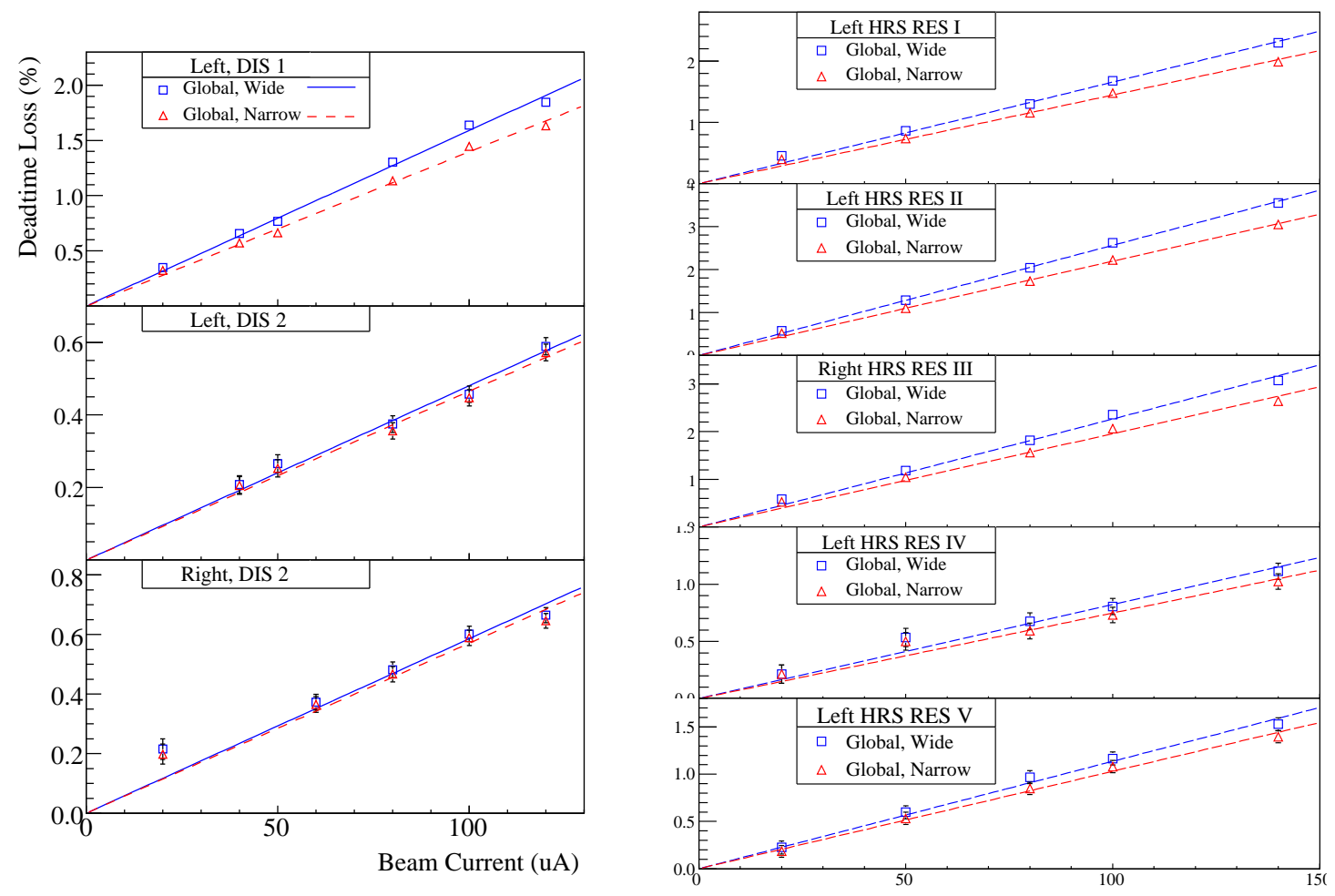

Figure 3.15: Total deadtime results for all kinematics obtained from simulation, for narrow (red open triangles, fit by dashed red lines) and wide (blue open squares, fit by solid blue lines) paths. 
The PID performances of the Cerenkov detector and the lead glass counter can be studied independently because the two detectors are based on different mechanisms and their PID efficiencies are not correlated. When investigating $\eta_{e}$ and $\eta_{\pi}$ for one of the detectors, we can use sample events selected by the other and calculate how many of the samples end up in the final trigger. The overall electron detection efficiency and pion rejection factor is the product the corresponding variables of the two detectors.

During PVDIS, the PID performance was closely monitored using on-line analysis of the HRS data. Specific PID runs with the VDCs turned on, which can provide more detailed diagnose of the PID performance, were taken on a daily basis. More complete and in-depth off-line analysis was performed after the experiment [65], the results of which are reported in [59].

\subsection{Calibration of the HRS Optics}

To accurately determine the kinematics $\left(Q^{2}, x, W\right)$ of each event, one must reconstruct precisely the position of the reaction point, the scattering angle, and scattered electron's momentum from the particle trajectory detected. These quantities were determined by calibration of the transport functions, or optics, of the HRS. The procedure and results of the optics calibration is described in this section.

\subsubsection{Optics Calibration Procedure and the Resulting Sys- tematic Uncertainties}

As described in Section 2.5.2.1, the VDCs were turned off during production runs. They were only turned on for optics calibration runs. The VDCs provided precise information on the particle trajectory, from which the hit position and angles at the focal plane $(x, \theta, y, \phi, l, \delta)$ can be determined [66]. The next step is to reconstruct 
the interaction position, angle, and momentum at the target from these focal plane variables, i.e., to determine the inverse of the HRS optical transport matrix. In practice, instead of a matrix operation, a set of tensors up to the 5th order were used to calculate the target variables from the focal plane values.

The target coordinates of the scattering event, $\left(x_{t g}, y_{t g}, \theta_{t g}, \phi_{t g}\right)$, are defined in the target coordinate system (TCS) [66] with respect to the spectrometer central ray direction, see Fig. 3.16. Here the angles $\theta_{t g}$ and $\phi_{t g}$ refer to the tangent of the vertical and horizontal angles relative to the HRS central ray. The spectrometer pointing $D$ is the distance at which the spectrometer misses the Hall center in the direction perpendicular to the spectrometer central ray. The sieve plane corresponds to the entrance of the spectrometer which is located at $L=1.12 \mathrm{~m}$ from the TCS origin. The particle hit position and the angles at the sieve plane can be directly calculated from the focal plane variables.

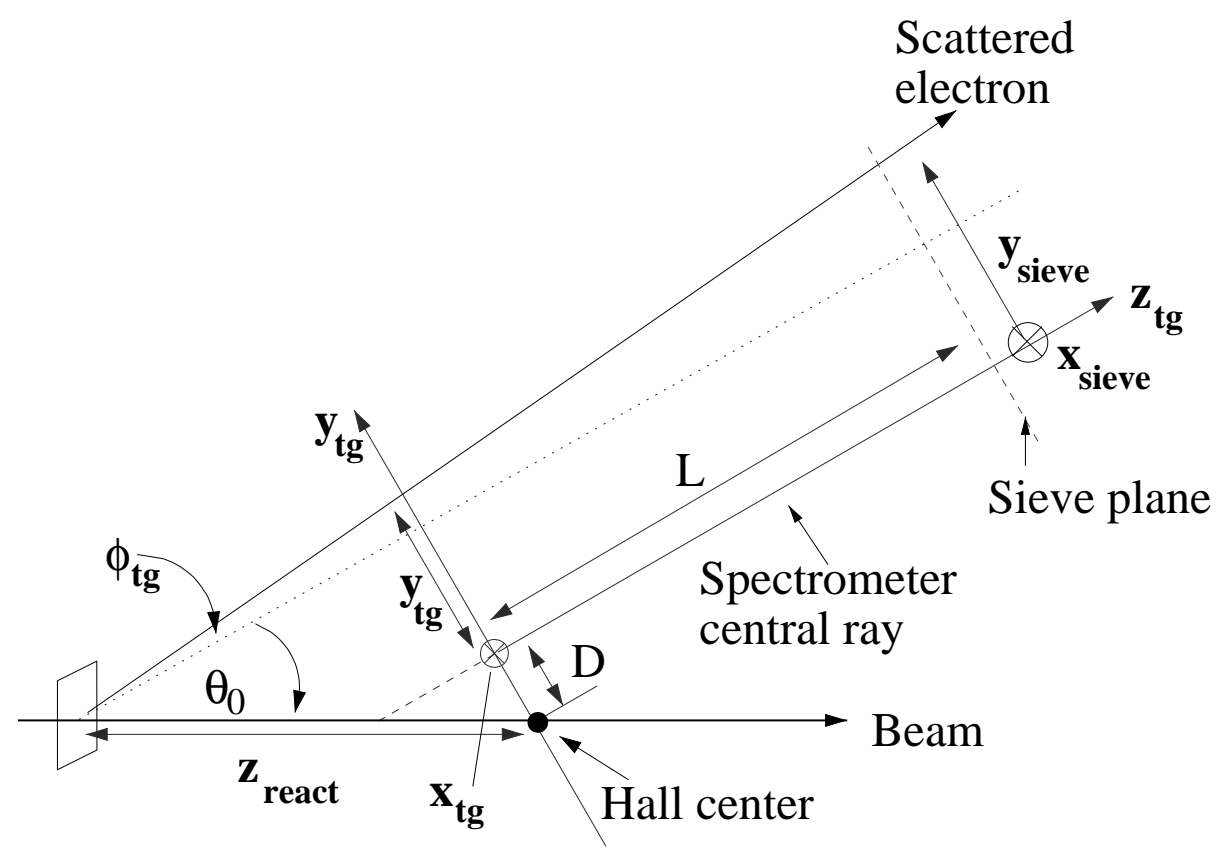

Figure 3.16: Top view of the target coordinate system (TCS) $\left(x_{t g}, y_{t g}, z_{t g}\right)$ and the sieve plane coordinate system $\left(x_{\text {sieve }}, y_{\text {sieve }}\right)$. 
In general, the optics calibration can be divided into three calibration steps:

1. Calibration of the interaction vertex position along the target, $z_{\text {react }}$, which is related to $y_{t g}, \phi_{t g}$ in the TCS as well as the pointing $D$ of the spectrometer. The vertex calibration was done by taking data on the multi-foil carbon target with known foil positions. The foil positions were determined from data using the HRS optics matrix, the focal plane variables, and $D$. The calibration precision on $z_{\text {react }}$ in the direction perpendicular to the spectrometer central ray is given by

$$
\Delta\left(z_{\text {react }} \sin \theta_{0}\right)=\sqrt{(\Delta D)^{2}+\left(\Delta z_{\text {foil }} \sin \theta_{0}\right)^{2}+\left(\Delta z_{\text {foil data }} \sin \theta_{0}\right)^{2}} .
$$

Then, the uncertainty in the scattering angle due to vertex calibration is

$$
\Delta \phi_{t g}=\Delta\left(z_{\text {react }} \sin \theta_{0}\right) / L
$$

Here $\Delta z_{\text {foil }}= \pm 2.5 \mathrm{~mm}$ is the uncertainty of the actual foil position caused by possible shifts of the target ladder during the target cool-down. The precision of $D$ can be obtained from a spectrometer pointing survey with a typical precision of $\pm 0.5 \mathrm{~mm}$. If a survey was not available, the value of $D$ can be derived from surveys performed at a previous spectrometer angle setting. In this case, one compares the multi-carbon-foil data before and after the spectrometer rotation. If the observed shifts in $z$ in all foil positions can be explained consistently by a global change in $D$, then the shift is added to the value of $D$ from the previous survey and the uncertainty of $D$ is taken as $\pm 0.5 \mathrm{~mm}$. If neither carbon foil data nor a survey was available, $\Delta D$ is taken to be $\pm 5 \mathrm{~mm}$ which is the limit of how much the spectrometer can physically miss the Hall center. The quantity 
$\Delta z_{\text {foil data }}$ is $\pm 0.1 \mathrm{~mm}$ or the observed discrepancy in the foil position between calibration data and the expected values, whichever is larger.

2. Calibration of the scattering angle $\theta_{t g}, \phi_{t g}$. This was done by inserting a so-called "sieve slit" plate - a 0.5-mm thick tungsten plate with an array of pinholes - at the entrance of the spectrometer. Reconstruction of hole positions depends on the angle elements of the optical matrix. The calibration precision is determined by the knowledge of the actual hole positions $x_{\text {hole }}, y_{\text {hole }}$ w.r.t. the center of the spectrometer, and how well the reconstructed hole positions $x_{\text {hole data }}, y_{\text {hole data }}$ agree with the expected values.

The most straightforward way to determine $x_{\text {hole }}, y_{\text {hole }}$ is by a survey of the sieve slit plate. The survey uncertainty is $\pm 0.5 \mathrm{~mm}$ for both directions. If no survey was available, but there was no work done on the sieve slit plate (such as taking it off and putting it back on the HRS entrance), from past experience it was found that the horizontal position $y_{\text {hole }}$ is highly reproducible, to $\pm 0.1 \mathrm{~mm}$, and the vertical position $x_{\text {hole }}$ is reproducible to $\pm 0.5 \mathrm{~mm}$ due to the fact that this is the direction in which the sieve plate is moved into or out of the HRS entrance. In this case, results from earlier surveys can be used with these additional uncertainties added. The angle uncertainties from sieve slit calibrations are:

$$
\begin{aligned}
& \Delta \theta_{t g}=\sqrt{\left(\Delta x_{\text {hole }}\right)^{2}+\left(\Delta x_{\text {hole data }}\right)^{2}} / L, \\
& \Delta \phi_{t g}=\sqrt{\left(\Delta y_{\text {hole }}\right)^{2}+\left(\Delta y_{\text {hole data }}\right)^{2}} / L,
\end{aligned}
$$

where the in-plane angle $\phi_{t g}$ affects the scattering angle $\theta$ directly, while the out-of-plane angle $\theta_{t g}$ affects $\theta$ only in the second order and the effect is small. If no sieve slit data were taken, the angle calibration of a preceding experiment 
can be used based on the high reliability of the HRS. In this case, an additional $\pm 0.5 \mathrm{mrad}$ of uncertainty should be added to both $\Delta \theta_{t g}, \Delta \phi_{t g}$ to account for possible changes in the optics.

3. Momentum calibration: The most precise way to calibrate the momentum is to use elastic scattering from a carbon or the proton inside a water target. With a water target, the relative momentum $\delta \equiv d p / p$ with $p$ the HRS central momentum setting can be determined to $\pm 1 \times 10^{-4}$. Due to the high beam energy used, elastic measurement was not possible for this experiment. However, water target calibration was performed during the preceding experiment (HAPPEXIII) [17] and due to the high stability of the HRS magnets and transport system, one expect an uncertainty of $\delta= \pm 5 \times 10^{-4}$ for this experiment.

The three calibration steps described above are in general treated as independent from others, i.e., matrix elements related to position reconstruction have little dependence on those related to angle reconstruction, etc. For all calibration methods above, the optics tensor coefficients were determined from a $\chi^{2}$ minimization procedure in which the events were reconstructed as close as possible to the known position of the corresponding foil target or the sieve-slit hole.

\subsubsection{Optics Calibration Results}

During the PVDIS experiment, there were seven kinematics settings in total and one of them was carried out on both Left and Right HRS, thus there were a total of eight HRS+kinematics combinations: Left HRS DIS \#1, Left and Right HRS DIS \#2, Left HRS Resonance (RES) I, Left RES II, Right HRS RES III, Left HRS RES IV, and Left HRS RES V. Either vertex or angle calibrations, or both, were carried out for all eight settings except RES IV and V. The vertex calibration for Left DIS\#1 and 
the angle calibration results for Left RES II are shown in Fig. 3.17.

\subsubsection{2 $Q^{2}$ Uncertainties}

The $Q^{2}$ of each event can be calculated using Equation (1.1.1). The uncertainty in $Q^{2}$ is determined by the uncertainties in the scattering angle $\theta, E$ and $E^{\prime}$, but is dominated by the scattering angle uncertainty. The scattering angle is calculated as $[43]$ :

$$
\theta=\cos ^{-1}\left(\frac{\cos \theta_{0}-\phi_{t g} \sin \theta_{0}}{\sqrt{1+\theta_{t g}^{2}+\phi_{t g}^{2}}}\right)
$$

thus calibration of the horizontal angle $\phi_{t g}$ dominates the angle uncertainty. The total uncertainty on the scattering angle is the combination of the vertex calibration Eqs. (3.9.1-3.9.2) and $\Delta \phi_{t g}$ from the angle calibration:

$$
\Delta \theta \approx \sqrt{(\Delta D / L)^{2}+\left(\Delta z_{\text {foil }} \sin \theta_{0} / L\right)^{2}+\left(\Delta z_{\text {foil data }} \sin \theta_{0} / L\right)^{2}+\left(\Delta \phi_{t g}\right)^{2}} .
$$

where $\Delta \phi_{t g}$ is either from Eq. (3.9.4) if a sieve slit calibration was available, or from previous calibrations with a $0.5 \mathrm{mrad}$ additional uncertainty added, and the drift distance $L=1.12 \mathrm{~m}$ as in Fig. 3.16.

For some settings during PVDIS, there were both angle and vertex calibrations (Left RES I and II), or only the vertex but not the angle calibration (Left DIS\#1, Left DIS\#2, Right DIS\#2, Right RES III), or neither (Left RES IV and V). For both vertex and angle calibrations, the optics database and some survey results from the HAPPEX-III experiment that ran immediately before this experiment were used. Taking all uncertainties into account, the uncertainty in $Q^{2}$ due to HRS optics calibration is summarized in Table 3.6. 

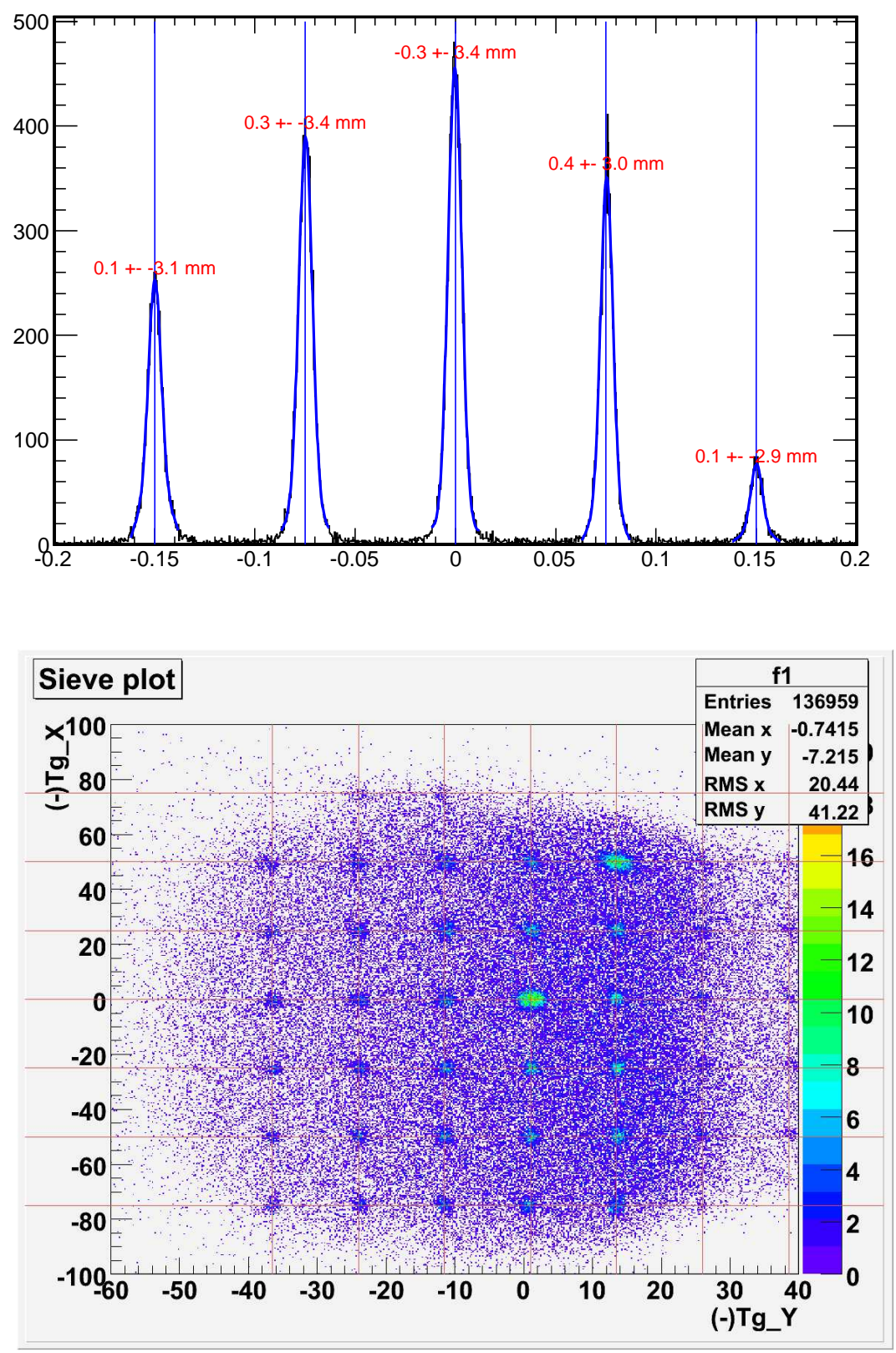

Figure 3.17: Top: vertex reconstruction for Left DIS\#1 (left), where the observed foil position miss the actual values by $\Delta z_{\text {foil data }} \leqslant 0.4 \mathrm{~mm}$. Bottom: reconstruction of the sieve hole positions (scattered points) compared to the expected positions (grids), for Left RES II. No obvious discrepancy is seen. The axes are oriented such that the sieve hole pattern is as if viewed when facing the spectrometer entrance. Two of the sieve holes are larger than others to allow identifying the center and the orientation of the sieve plate. 


\begin{tabular}{|c|c|c|c|c|c|c|c|c|}
\hline HRS & \multicolumn{6}{|c|}{ Left HRS } & \multicolumn{2}{|c|}{ Right HRS } \\
\hline Kinematics & DIS\#1 & RES V & RES IV & DIS\#2 & Res I & Res II & DIS\#2 & Res III \\
\hline$\theta_{0}\left({ }^{\circ}\right)$ & 12.9 & 14.0 & 15.0 & 20 & 12.9 & 12.9 & 20 & 12.9 \\
\hline$E_{b}(\mathrm{GeV})$ & 6.067 & 6.067 & 6.067 & 6.067 & 4.867 & 4.867 & 4.867 & 4.867 \\
\hline$E_{0}^{\prime}(\mathrm{GeV})$ & 3.66 & 3.66 & 3.66 & 2.63 & $4.0^{a}$ & 3.66 & 2.63 & 3.1 \\
\hline HRS pointing survey? & $\mathrm{Y}$ & $\mathrm{N}$ & $\mathrm{N}$ & $\mathrm{Y}$ & $\mathrm{N}$ & $\mathrm{N}$ & $\mathrm{Y}$ & $\mathrm{N}$ \\
\hline$\delta D($ survey $)(\mathrm{mm})$ & 0.5 & & & 0.5 & & & 0.5 & \\
\hline Carbon multi foil data available? & $\mathrm{Y}$ & $\mathrm{N}$ & $\mathrm{N}$ & $\mathrm{Y}$ & $\mathrm{Y}$ & $\mathrm{Y}$ & $\mathrm{Y}$ & $\mathrm{Y}$ \\
\hline$\delta D$ (from data, no survey) (mm) & & & & & 0.5 & 0.5 & 0.5 & 0.5 \\
\hline$\delta D($ no survey, no data $)(\mathrm{mm})$ & & 5.0 & 5.0 & & & & & \\
\hline$\delta z_{\text {foil data }}(\mathrm{mm})$ & 0.4 & $\mathrm{~N} / \mathrm{A}$ & $\mathrm{N} / \mathrm{A}$ & 0.4 & 2.0 & 0.3 & 0.7 & 1.1 \\
\hline$\delta z_{\text {foil }}$ & 2.5 & $\mathrm{~N} / \mathrm{A}$ & $\mathrm{N} / \mathrm{A}$ & 2.5 & 2.5 & 2.5 & 2.5 & 2.5 \\
\hline $\begin{array}{l}\Delta \theta \text { from } \quad \text { vertex } \\
(\mathrm{mrad}), \mathrm{Eq} \cdot(3.9 .2)\end{array}$ & 0.676 & 4.464 & 4.464 & 0.893 & 0.779 & 0.672 & 0.901 & 0.704 \\
\hline sieve survey & $\mathrm{N}$ & $\mathrm{N}$ & $\mathrm{N}$ & $\mathrm{N}$ & $\mathrm{N}$ & $\mathrm{N}$ & $\mathrm{N}$ & $\mathrm{N}$ \\
\hline sieve data & $\mathrm{N}$ & $\mathrm{N}$ & $\mathrm{N}$ & $\mathrm{N}$ & $\mathrm{Y}$ & $\mathrm{Y}$ & $\mathrm{N}$ & $\mathrm{N}$ \\
\hline$\Delta x_{\text {hole }}$, from prior survey $(\mathrm{mm})$ & 0.51 & 0.51 & 0.51 & 0.51 & 0.51 & 0.51 & 0.51 & 0.51 \\
\hline$\Delta x_{\text {hole data }}(\mathrm{mm})$ & 0.1 & $\mathrm{~N} / \mathrm{A}$ & $\mathrm{N} / \mathrm{A}$ & 0.1 & 0.1 & 0.1 & 0.1 & 0.1 \\
\hline additional $\Delta \phi_{t g}(\mathrm{mrad})$ & $0.5^{b}$ & $0.5^{b}$ & $0.5^{b}$ & $0.5^{b}$ & & & $0.5^{c}$ & $0.5^{c}$ \\
\hline $\begin{array}{l}\Delta \theta \text { from angle calibration (mrad), } \\
\text { Eq. }(3.9 .4)\end{array}$ & 0.682 & 0.676 & 0.676 & 0.682 & 0.464 & 0.464 & 0.676 & 0.676 \\
\hline Total $\Delta \theta(\mathrm{mrad})$ & 0.960 & 4.515 & 4.515 & 1.124 & 0.907 & 0.816 & 1.134 & 0.976 \\
\hline Total $\Delta \theta / \theta(\%)$ & 0.426 & 1.848 & 1.725 & 0.322 & 0.403 & 0.363 & 0.325 & 0.434 \\
\hline$\Delta E_{0}^{\prime} / E_{0}^{\prime}$ & & & & $5 \times 1$ & & & & \\
\hline Total $\Delta Q^{2} / Q^{2}(\%)^{d}$ & 0.853 & 3.696 & 3.449 & 0.644 & 0.805 & 0.725 & 0.650 & 0.867 \\
\hline
\end{tabular}

${ }^{a}$ Magnets mismatched, see text;

${ }^{b}$ Due to using sieve calibration taken at Left RES\#3;

${ }^{c}$ Due to using optics database from HAPPEX-III;

${ }^{d}$ Including uncertainties due to both scattering angle $\Delta \theta$ and momentum $\Delta E^{\prime}$, but is dominated by the former.

Table 3.6: PVDIS $\mathrm{Q}^{2}$ uncertainty due to optics calibration. For each HRS, the kinematics are shown from left to right in the chronological order. 


\subsection{Hall A Monte Carlo}

As described in the previous sections, the kinematics $\left(Q^{2}, W, x\right)$ of the scattering event can be fully determined from the beam energy and the measured angle and momentum of the events, and their uncertainties derived from the optical calibration. To fully understand these event kinematics, a simulation package called "HAMC" (Hall A Monte Carlo) was used to simulate the transport function and the acceptance of HRS, and it is expected that the simulated values should agree with the measured ones within their uncertainties.

In HAMC, events were generated with a uniform distribution in the solid angle $d \Omega=\sin (\theta) d \theta d \phi$, then transported through the HRS magnets using a set of polynomials that model the electrons' trajectories through the magnetic fields. Events that passed all magnet entrance and exit apertures would fall within the HRS acceptance and be recorded in the simulated sample. Also accounted for in HAMC were effects from multiple scattering in the target material, energy loss due to external and internal Bremsstrahlung and ionization loss, and resolutions of the VDC wires. The physical differential cross section $d^{2} \sigma /\left(d E^{\prime} d \Omega\right)$ and the parity violation asymmetry were calculated for each simulated event.

In general, the acceptance of the HRS is defined by combining the opening geometry of the intermediate apertures, the nominal settings of which were documented in Ref. [43]. In real experiments, however, the edges of the openings are not well defined because events falling on the edge may correspond to electrons scattering from the aperture's material. The real acceptance can thus be different from the nominal settings. The HRS acceptance of the simulation was fine-tuned by evaluating the cross-section-weighted event distributions at different apertures and adjusting slightly the aperture edges to match the data. This process is illustrated in Fig. 3.18. 

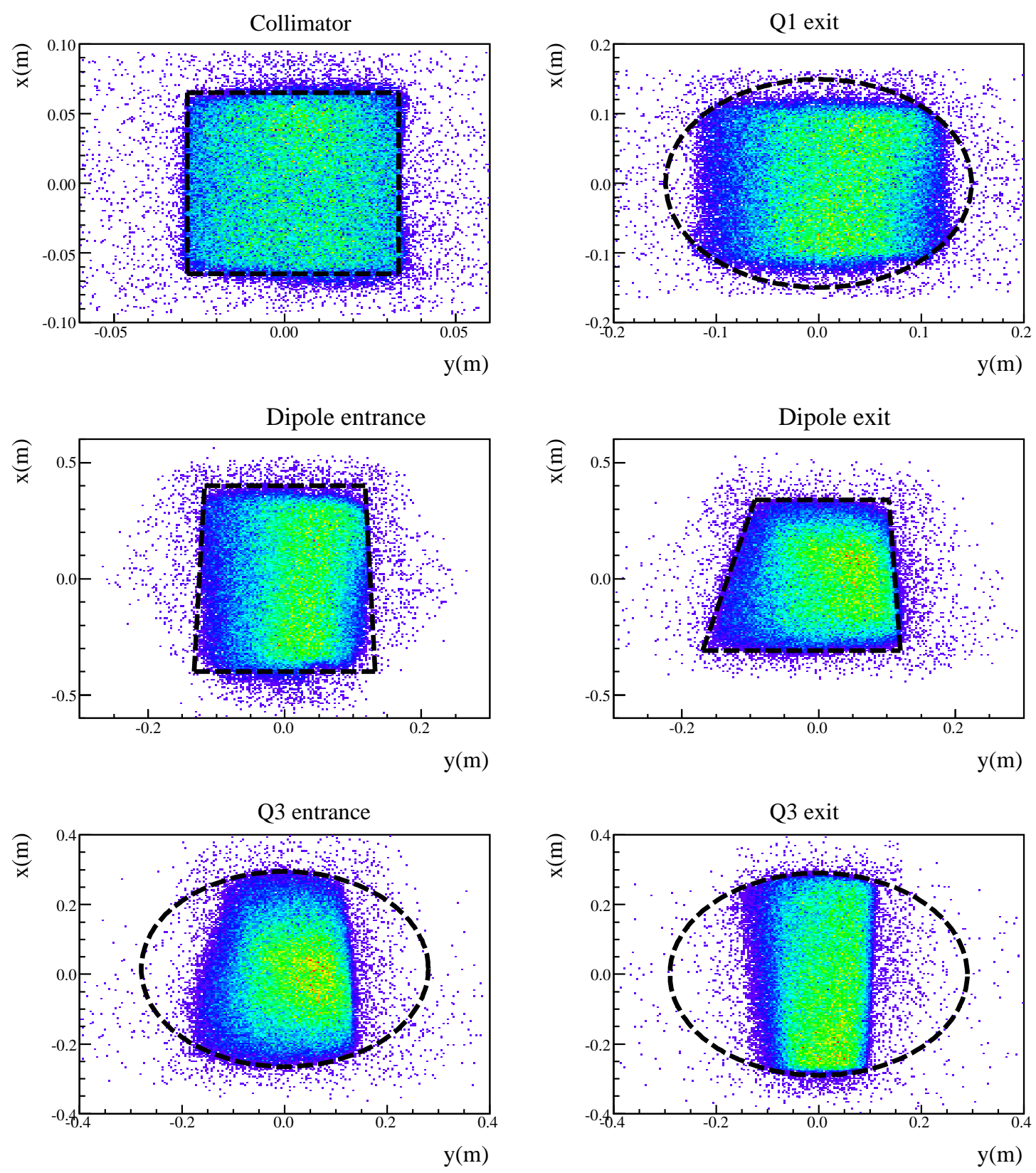

Figure 3.18: Fine-tuning of the HRS acceptance in HAMC. Event distributions from data are plotted at the collimator (entrance of the HRS $Q_{1}$ ), $Q_{1}$ exit, and entrances and exits of the dipole, $Q_{2}$ and $Q_{3}$. Black dashed lines show the position, shape, and size of apertures determined by matching to the data distributions, which were then used as aperture or acceptance cuts in HAMC. The axes are oriented such that the distributions are as viewed along the particle trajectory, with $y$ the horizontal and $x$ the vertical (dispersive) directions, respectively. 
Once all magnet apertures were optimized by data, the kinematics $\left(Q^{2}, x\right)$ can be calculated in HAMC from Equations (1.1.1,1.1.3) using the simulated $\theta$ and $E^{\prime}$. For the incident electron's energy $E$, the values of 6.067 and $4.867 \mathrm{GeV}$ were used for the initial beam, minus an average energy loss of $3 \mathrm{MeV}$ to account for the effect of passing through all material along the beamline up to the target center. Differences in $Q^{2}$ and $x$ between simulation and values reconstructed from the actual data provide a measure of how well we understand the measurement, and should be consistent with the uncertainty estimation of Table 3.6.

Figure 3.19 shows comparisons between data and simulation for all target variables, $Q^{2}$ and $x$ for Left HRS DIS \#1 and Right HRS DIS \#2. A summary of the comparison for all kinematics is given in Table 3.7. The observed differences in $Q^{2}$ are consistent with the uncertainties shown in Table 3.6.

\begin{tabular}{c|c|c|c|c|c|c}
\hline \multirow{2}{*}{ Kinematics } & \multicolumn{3}{|c|}{ HAMC } & \multicolumn{3}{c}{ data } \\
\cline { 2 - 7 } & $\begin{array}{c}\left\langle Q^{2}\right\rangle \\
(\mathrm{GeV} / \mathrm{c})^{2}\end{array}$ & $\langle x\rangle$ & $\begin{array}{c}\left\langle W^{2}\right\rangle \\
\mathrm{GeV}^{2}\end{array}$ & $\begin{array}{c}\left\langle Q^{2}\right\rangle \\
(\mathrm{GeV} / \mathrm{c})^{2}\end{array}$ & $\langle x\rangle$ & $\begin{array}{c}\left\langle W^{2}\right\rangle \\
\mathrm{GeV}^{2}\end{array}$ \\
\hline Left HRS DIS\#1 & 1.084 & 0.241 & 4.294 & 1.085 & 0.241 & 4.297 \\
Left+Right HRS DIS\#2 & 1.892 & 0.294 & 5.424 & 1.901 & 0.295 & 5.430 \\
Left HRS RES I & 0.956 & 0.571 & 1.600 & 0.950 & 0.571 & 1.595 \\
Left HRS RES II & 0.832 & 0.336 & 2.528 & 0.831 & 0.335 & 2.530 \\
Right HRS RES III & 0.745 & 0.225 & 3.443 & 0.757 & 0.228 & 3.450 \\
Left HRS RES IV & 1.456 & 0.324 & 3.925 & 1.472 & 0.326 & 3.923 \\
Left HRS RES V & 1.268 & 0.282 & 4.109 & 1.278 & 0.283 & 4.122 \\
\hline
\end{tabular}

Table 3.7: Comparison of $Q^{2}, x$, and $W^{2}$ between HAMC and data for all kinematics. The Left and the Right DIS\#2 have been combined.

\subsection{Electromagnetic Radiative Correction}

Electrons undergo radiative energy losses due to interactions such as internal and external bremsstrahlung and ionization loss, both before and after the scattering. 

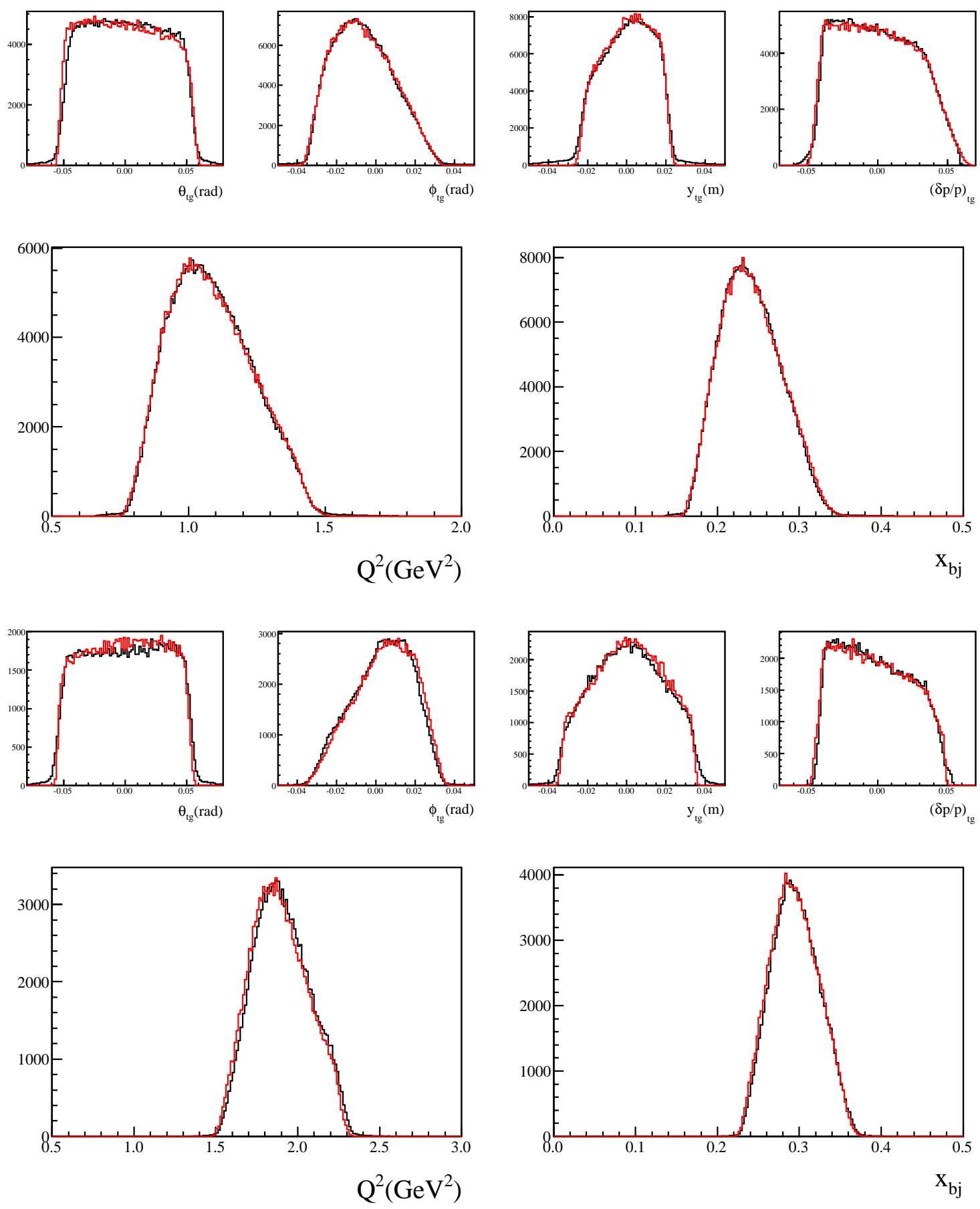

Figure 3.19: Comparison between HAMC (red) and data (black). From top to bottom: target variables $-\theta_{\mathrm{tg}}, \phi_{\mathrm{tg}}, y_{\mathrm{tg}}$ and $(\delta p / p)_{\mathrm{tg}}-$ for Left HRS DIS \#1; $Q^{2}$ and $x$ for Left HRS DIS\#1; target variables for Right HRS DIS\#2; $Q^{2}$ and $x$ for Right HRS DIS\#2. 
This causes two effects on the measurement: 1) There is a small beam depolarization effect associated with the energy loss of incident electrons; 2) the energy loss of both incident and scattered electrons would cause a difference between the kinematics reconstructed from the detected signals and what really happened at the interaction vertex. We discuss these two effects separately.

\subsubsection{Beam Depolarization Effect in Bremsstralung}

The depolarization of electron from bremsstralung radiation was calculated based on Eq.(9.11) of Ref. [78]:

$$
D\left(\vec{p}_{1}, \vec{\zeta}_{1}\right)=\frac{k^{2}\left[\psi_{1}-\zeta_{1 z}^{2}\left(\psi_{1}-\frac{2}{3} \psi_{2}\right)\right]}{\left(\epsilon_{1}^{2}+\epsilon_{2}^{2}\right) \psi_{1}-\frac{2}{3} \epsilon_{1} \epsilon_{2} \psi_{2}}
$$

where $\epsilon_{1,2}$ are the energy of the electron before and after bremsstralung in unit of the electron mass $m_{e} c^{2}, k$ is the bremsstralung photon energy in unit of $m_{e} c^{2}, \vec{\zeta}$ is the polarization vector of the electron with $\zeta_{1 z}=1$ for longitudinally polarized electrons, and $\psi_{1,2}$ are given in the "complete screening" limit by

$$
\begin{aligned}
& \psi_{1}=4 \ln \left(111 Z^{-1 / 3}\right)+2-4 f(Z)=4\left[\ln \left(183 Z^{-1 / 3}\right)-f(Z)\right], \\
& \psi_{2}=4\left[\ln \left(183 Z^{-1 / 3}\right)-f(Z)\right]-\frac{2}{3} .
\end{aligned}
$$

The function $f(Z)$ is

$$
f(Z)=a^{2} \sum_{n=1}^{\infty} \frac{1}{n\left(n^{2}+a^{2}\right)},
$$

with $a=\left(Z e^{2} / \hbar / c\right)$.

The "complete screening" limit is defined as $\beta_{i} \xi / \delta \gg 1$ where $\beta_{i}=\left(Z^{1 / 3} / 121\right) b_{i}$ 
with $b_{1}=6, b_{2}=1.2$ and $b_{3}=0.3 ; \xi \equiv 1 /\left(1+u^{2}\right)$ with $u=p_{1} \theta_{1}$; and $\delta \equiv k /\left(2 \epsilon_{1} \epsilon_{2}\right)$. Here $\vec{p}_{1}, \vec{p}_{2}$ are momentum of the electron before and after bremsstralung in unit of $m_{e} c$, and $\theta_{1}, \theta_{2}$ are the angles between $\vec{p}_{1}, \vec{p}_{2}$ and the photon $\vec{k}$, respectively. Because for high energy electrons $\theta_{1}$ is very small, $u \approx 0$ and $\xi \approx 1$. Putting all notations together, the complete screening limit is

$$
\frac{\beta_{i} \xi}{\delta}=\frac{\frac{Z^{1 / 3}}{121} b_{i}}{\left(1+\epsilon_{1}^{2} \theta_{1}^{2}\right) \frac{k}{2 \epsilon_{1} \epsilon_{2}}} \approx \frac{\frac{Z^{1 / 3}}{121} b_{i}}{\frac{k}{2 \epsilon_{1} \epsilon_{2}}+\frac{1}{2} k \theta_{1}^{2}} \gg 1
$$

where the approximation is valid if $k \ll \epsilon_{1}$ and the complete screening condition is satisfied if $\epsilon_{1} \gg 1$. For the $6-\mathrm{GeV}$ beam used in this experiment, $\epsilon_{1} \approx 12000$ and $k \ll \epsilon_{1}$, therefore the complete screening limit can be used.

We define a depolarization correction

$$
f_{\text {depol }}=\frac{\left\langle A_{e} D\right\rangle}{\left\langle A_{e}\right\rangle}
$$

where $D$ is the beam depolarization factor (with zero depolarization corresponding to $D=100 \%)$ and the average of a quantity $\langle a\rangle\left(a=A_{e}\right.$ or $\left.A_{e} D\right)$ is taken over the spectrometer acceptance and the cross section $\sigma$ :

$$
\langle a\rangle \equiv \frac{\int a \cdot \sigma \cdot(\text { acceptance })}{\int \sigma \cdot(\text { acceptance })} .
$$

The measured asymmetry should be corrected as

$$
A^{\text {depol-corrected }}=A_{e}^{\text {meas }}\left(1+\bar{f}_{\text {depol }}\right),
$$

where $\bar{f}_{\text {depol }} \equiv\left(1 / f_{\text {depol }}\right)-1 \approx\left\langle A_{e}\right\rangle /\left\langle A_{e} D\right\rangle-1$. HAMC simulation was done to determine the value of $\bar{f}_{\text {depol }}$ and the results are shown in Table 3.8. 


\begin{tabular}{c|c|c|c|c|c|c|c}
\hline \hline Kinematics & DIS\#1 & DIS\#2 & RES I & RES II & RES III & RES IV & RES V \\
\hline$f_{\text {depol }}$ & $0.096 \%$ & $0.209 \%$ & $0.005 \%$ & $0.028 \%$ & $0.093 \%$ & $0.061 \%$ & $0.081 \%$ \\
\hline \hline
\end{tabular}

Table 3.8: Beam depolarization correction $\bar{f}_{\text {depol }}$ for all kinematics.

\subsubsection{Corrections for Vertex versus Detected Kinematics}

Due to energy losses of the electrons, the kinematics at the interaction vertex is not the same as those calculated from the initial beam energy and the electron's momentum detected by the spectrometer. This effect is illustrated in Fig. 3.20: since the shift

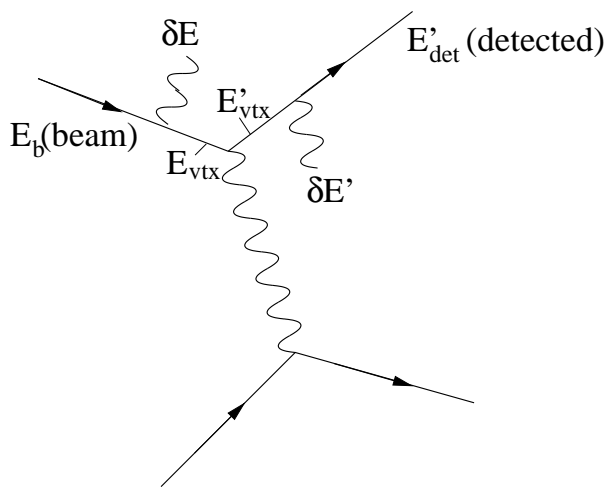

Figure 3.20: Kinematics used in HAMC to correct energy losses $\delta E$ and $\delta E^{\prime}$, respectively for the incoming and outgoing electrons. The kinematics reconstructed from the data corresponds to $E_{\text {beam }}$ and $E_{\text {det }}^{\prime}$, while the vertex kinematics corresponds to $E_{v}$ and $E_{v}^{\prime}$.

between detected and vertex kinematics relies heavily on the experimental setup, it is desired to correct the measured asymmetry for this effect such that the corrected values can be compared to theoretical expectations in a less ambiguous way. This correction factor is defined as:

$$
1+\bar{f}_{\mathrm{rc}}=\frac{A\left(\left\langle Q_{\mathrm{det}}^{2}\right\rangle,\left\langle x_{\mathrm{det}}\right\rangle\right)}{\left\langle A\left(Q_{\mathrm{vtx}}^{2}, x_{\mathrm{vtx}}\right)\right\rangle}
$$


and is applied to the measured asymmetry as:

$$
A_{e}^{\text {rad-corrected }}=A_{e}^{\text {meas }}\left(1+\bar{f}_{r c}\right) .
$$

Here $A\left(\left\langle Q_{\mathrm{det}}^{2}\right\rangle,\left\langle x_{\mathrm{det}}\right\rangle\right)$ is the asymmetry calculated at the cross-section- and acceptanceweighted values [see Eq. (3.11.7)] of $Q_{\text {det }}^{2}$ and $x_{\text {det }}$, evaluated from the initial beam energy and the detected electrons momentum, and $\left\langle A\left(Q_{\mathrm{vtx}}^{2}, x_{\mathrm{vtx}}\right)\right\rangle$ is the asymmetry still averaged over all detected electrons following Eq. (3.11.7), but now calculated using the vertex kinematics $Q_{\mathrm{vtx}}^{2}$ and $x_{\mathrm{vtx}}$ of each event. Since the value $\left\langle A\left(Q_{\mathrm{vtx}}^{2}, x_{\mathrm{vtx}}^{2}\right)\right\rangle$ is the expected value of what was actually measured in the experiment $\left(A_{e}^{\text {meas }}\right)$, the result $A_{e}^{\mathrm{rad}-\text { corrected }}$ can be treated as the value corresponding to $\left\langle Q_{\mathrm{det}}^{2}\right\rangle$ and $\left\langle x_{\mathrm{det}}\right\rangle$. The value of $A_{e}^{\text {rad-corrected }}$ can thus be compared with theoretical calculations evaluated at $\left\langle Q_{\mathrm{det}}^{2}\right\rangle$ and $\left\langle x_{\mathrm{det}}\right\rangle$ to extract physics results.

The radiative correction was evaluated using HAMC which calculates both the numerator and the denominator of Eq. (3.11.9). The treatment of radiative effects was based on the prescription of Mo \& Tsai [80]. The detailed procedure is described below:

For each simulated event, the scattering angle $\theta$ and the momentum of the scattered electron $E_{\mathrm{vtx}}^{\prime}$ at the vertex were generated randomly. The energy loss of incoming and outgoing electrons $\delta E$ and $\delta E^{\prime}$ were then calculated using the formula given on page 5-7 of Ref. [81], which includes external bremsstralung, internal bremsstralung using the effective radiator formula, and ionization loss. Next, the incoming electron's energy at the vertex is calculated as $E_{\mathrm{vtx}}=E_{b}-\delta E$ where $E_{b}$ is the (fixed) initial beam energy and the detected momentum of the scattered electron calculated as $E_{\mathrm{det}}^{\prime}=E_{\mathrm{vtx}}^{\prime}-\delta E^{\prime}$. If $\theta$ and $E_{\mathrm{det}}^{\prime}$ falled within the spectrometer acceptance, the cross section and the PV asymmetry were calculated using both the detected $\left(E_{b}, E_{\text {det }}, \theta\right)$ 
and the vertex kinematics $\left(E_{\mathrm{vtx}}, E_{\mathrm{vtx}}^{\prime}, \theta\right)$ and were stored.

The vertex kinematics $\left(Q_{\mathrm{vtx}}^{2}, W_{\mathrm{vtx}}\right)$ calculated using $\left(E_{\mathrm{vtx}}, \theta, E_{\mathrm{vtx}}^{\prime}\right)$ is shown in Fig. 3.21 for the two DIS kinematics. One can see that the vertex kinematics of an event could fall into one of the following categories: $e-{ }^{2} \mathrm{H}$ elastic $(W$ below the proton mass, quasi-elastic ( $W \approx$ the proton mass), nucleon resonances $(1 \lesssim W<2 \mathrm{GeV})$, and DIS $(W>2 \mathrm{GeV})$. To evaluate the PV asymmetries for different vertex kine-
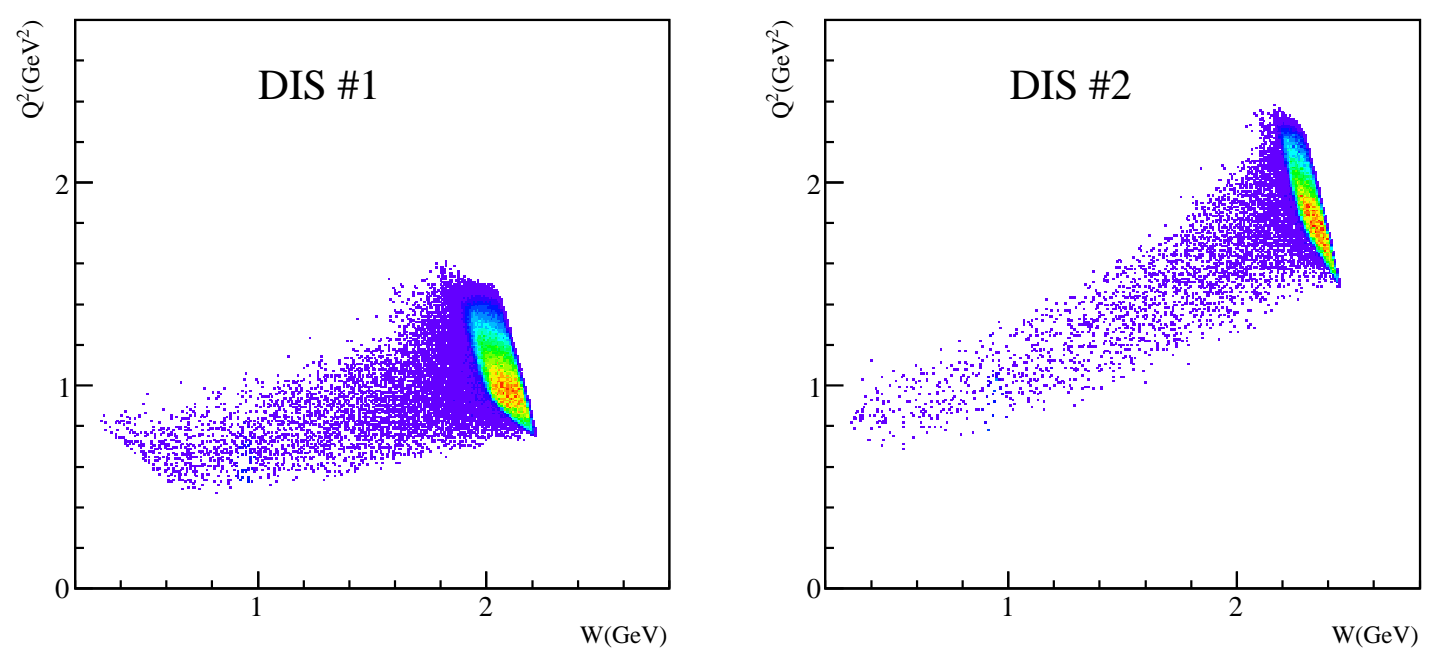

Figure 3.21: Simulated vertex kinematics of the two DIS kinematics \#1 (left) and \#2 (right).

matics, the following prescription was used:

1. For $e-{ }^{2} \mathrm{H}$ elastic scattering, the method from the SAMPLE experiment [82] was used, where the cross section was based on Ref. [83] and the PV asymmetry was based on a simple model that compares well to the calculation of Ref. [84]. The strange magnetic form factor $G_{M}^{s}$ in this method was taken to be zero.

2. For quasi-elastic scattering, the cross section and the asymmetry were calculated using the elastic scattering formula and elastic form factors for the neutron and the proton [see Section VII of Ref. [15]], then smeared for their Fermi motion 
following the algorithm of Ref. [74]. The quasi-elastic PV asymmetry was then calculated as $A_{d}^{\mathrm{qe}}=\left(A_{p} \sigma_{p}+A_{n} \sigma_{n}\right) /\left(\sigma_{p}+\sigma_{n}\right)$ where $A_{p(n)}, \sigma_{p(n)}$ are the elastic asymmetries and cross sections for the proton (neutron), respectively.

3. For the nucleon resonance region $(1 \lesssim W<2 \mathrm{GeV})$, the cross section was based on Ref. [74], and the asymmetries were calculated from three models: one theoretical model on the $\Delta(1232)$ only [85], a second theoretical model that covers almost the whole resonance region [86], and one "cross-sectionscaling model" where $A_{\text {res }}=\frac{\sigma_{\text {res }}}{\sigma_{\text {dis }}} A_{\text {dis }}$ was used. Here $A_{\text {dis }}$ was calculated from Eq. (1.2.4) with MSTW2008 PDFs [92], $\sigma_{\text {dis }}$ was calculated using the NMC fit of $F_{2}$ [73] structure functions and $R$ from Ref. [74], and $\sigma_{\text {res }}$ was from Ref. [74] which exhibits distinct resonance structures; The cross-section-scaling model was used only when the theoretical models do not cover the kinematics of a particular event.

4. For DIS ( $W>2 \mathrm{GeV}$ ), the cross section was calculated using Bosted's fits [74] and the PV asymmetry was calculated using Eqs. (1.2.4-1.2.6), the MSTW2008 PDFs [92] and the quark-parton model formula Eqs. (1.2.13), (1.2.11), (1.2.14), and (1.2.15). For $R$ in Eq. (1.2.13) again Ref. [74] was used.

The physics inputs to HAMC for $e-{ }^{2} \mathrm{H}$ elastic, quasi-elastic, DIS, as well as the cross sections were all based on existing data and the uncertainties are small. The uncertainty of the correction was thus dominated by that from the resonance asymmetry models. The validity of these models were evaluated by comparing the measured asymmetries from the resonance kinematics, RES I through IV, with calculations from these models. The kinematic coverage of resonance measurements is shown in Fig. 3.22. These resonance asymmetries were reported in Ref. [67], and it was found that the data agree well with both resonance models $[85,86]$ except RES I. Results 

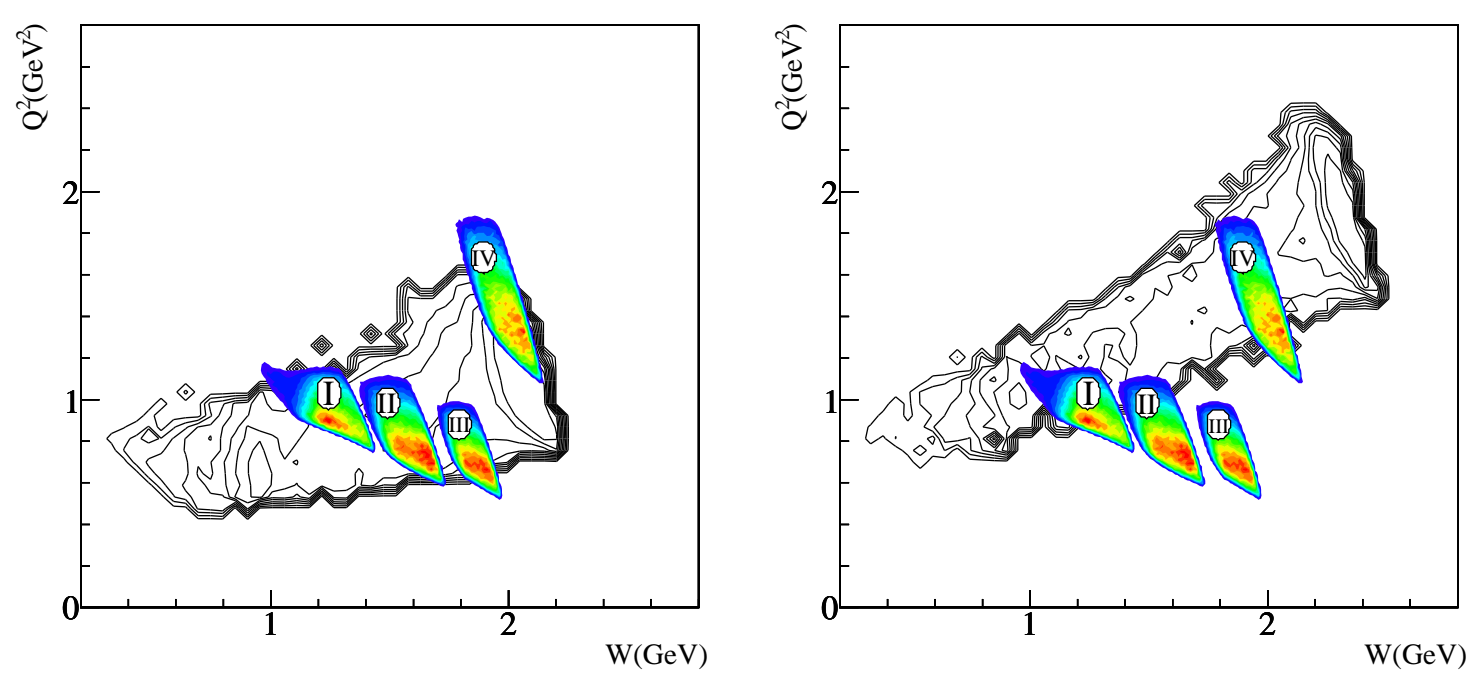

Figure 3.22: Kinematics coverage of the four resonance measurements (colored contours), together with the DIS kinematics (black contours).

at RES I agreed with the two models at the two standard deviation level. The uncertainty from the resonance models was taken to be either the observed difference between resonance data and model, or the statistical uncertainty of the resonance asymmetry measurement, whichever is larger. This gives different model uncertainties as follows:

- For $W^{2}<1.96(\mathrm{GeV})^{2}$ or the $\Delta(1232)$ region: RES I locates primarily in this region. The observed 25\% discrepancy between RES I data and the calculation was used as model uncertainty in this region;

- For $1.96<W^{2}<3.0(\mathrm{GeV})^{2}$ : RES II locates primarily in this region. Since the RES II asymmetry result agreed well with both models, the $10.0 \%$ statistical uncertainty of the RES II asymmetry was used as model uncertainty in this region.

- For $3.0<W^{2}<4.0(\mathrm{GeV})^{2}$ : Both RES III and IV locate in this region. Since the agreement with the calculations was well within the statistical uncertainties, 
the relative uncertainties for RES III and IV ( $8.9 \%$ and $15.4 \%$ respectively) were combined, and the resulting value of $7.7 \%$ was used as model uncertainty in this region.

For radiative corrections for DIS kinematics, the resonance models affect the denominator, but not the numerator of Eq. (3.11.9). Therefore the above model uncertainty affects directly the DIS corrections. These uncertainties were combined with the fractional events whose vertices fell within the corresponding $W$ region to estimate the uncertainty on $\left\langle A\left(Q_{\mathrm{vtx}}^{2}, x_{\mathrm{vtx}}\right)\right\rangle$ and $\bar{f}_{\mathrm{rc}}$. For radiative corrections for resonance kinematics, the resonance models affect both the denominator and the numerator of Eq. (3.11.9). The uncertainty of the model itself therefore cancels out in principle in the correction factor $\bar{f}_{\text {rc }}$. For resonance kinematics, a conservative $20 \%$ relative uncertainty was assigned to the value of $\bar{f}_{\mathrm{rc}}$.

The radiative correction factor $1+\bar{f}_{r c}$ obtained from the above procedure is shown in Table 3.9 for the two models separately. The average value of the two models were applied to the measured asymmetries of this experiment.

\subsubsection{Box Diagram Corrections}

Box diagram corrections refer to effects that arise when the electron simultaneously exchanges two bosons ( $\gamma \gamma, \gamma Z$, or $Z Z$ box) with the target, and they are dominated by the $\gamma \gamma$ and the $\gamma Z$ box diagrams. For PVES asymmetries, the box diagram effects include those from the interference between $Z$-exchange and the $\gamma \gamma$ box, the interference between $\gamma$-exchange and the $\gamma Z$ box, and the effect of the $\gamma \gamma$ box on the electromagnetic cross sections. It is expected that there is at least partial cancellation among these three terms. The box-diagram corrections were applied as

$$
A^{\text {box-corrected }}=\left(1+\bar{f}_{\text {box }}\right) A_{e} .
$$




\begin{tabular}{c|c|c|c|c|c}
\hline \hline Kinematics & Resonance Models & $\begin{array}{c}A\left(\left\langle Q_{\mathrm{det}}^{2}\right\rangle,\left\langle x_{\mathrm{det}}^{2}\right\rangle\right) \\
\mathrm{ppm}\end{array}$ & $\begin{array}{c}\left\langle A\left(Q_{\mathrm{vtx}}^{2}, x_{\mathrm{vtx}}^{2}\right)\right\rangle \\
\mathrm{ppm}\end{array}$ & $1+\bar{f}_{r c}$ & $\begin{array}{c}1+\bar{f}_{r c} \\
\text { average }\end{array}$ \\
\hline \multirow{2}{*}{ DIS \#1 } & Ref. [85] + c.s.-scaling & -88.6 & -86.8 & $1.021 \pm 0.02$ & $1.015 \pm 0.021$ \\
& Ref.[86] + c.s.-scaling & -88.6 & -87.8 & $1.009 \pm 0.02$ & \\
\hline \multirow{2}{*}{ DIS \#2 } & Ref. [85] + c.s.-scaling & -159.6 & -156.6 & $1.019 \pm 0.0043$ & $1.019 \pm 0.0043$ \\
& Ref.[86] + c.s.-scaling & -159.6 & -156.7 & $1.019 \pm 0.0043$ & \\
\hline \multirow{2}{*}{ RES I } & Ref. [85] + c.s.-scaling & -93.39 & -82.17 & $1.137 \pm 0.027$ & $1.1095 \pm 0.0352$ \\
& Ref.[86] + c.s.-scaling & -88.97 & -82.23 & $1.082 \pm 0.016$ & \\
\hline \multirow{2}{*}{ RES II } & Ref. [85] + c.s.-scaling & -65.47 & -65.46 & $1.0002 \pm 0.0000$ & $1.0205 \pm 0.0207$ \\
& Ref.[86] + c.s.-scaling & -71.07 & -68.28 & $1.0408 \pm 0.0082$ & \\
\hline \multirow{2}{*}{ RES III } & Ref. [85] + c.s.-scaling & -58.64 & -59.05 & $0.9930 \pm 0.0014$ & $1.0005 \pm 0.0076$ \\
& Ref.[86] + c.s.-scaling & -62.51 & -62.02 & $1.0079 \pm 0.0016$ & \\
\hline \multirow{2}{*}{ RES IV } & Ref. [85] + c.s.-scaling & -117.48 & -116.74 & $1.0063 \pm 0.0013$ & $1.0170 \pm 0.0112$ \\
& Ref.[86] + c.s.-scaling & -123.71 & -120.38 & $1.0276 \pm 0.0055$ & \\
\hline \multirow{2}{*}{ RES V } & Ref. [85] + c.s.-scaling & -103.87 & -101.43 & $1.0241 \pm 0.0048$ & $1.0134 \pm 0.0110$ \\
& Ref.[86] + c.s.-scaling & -103.87 & -103.60 & $1.0027 \pm 0.0005$ & \\
\hline \hline
\end{tabular}

Table 3.9: Radiative correction factors. For each kinematics, the simulated asymmetries using two resonance models are shown. In kinematic regions where the resonance models are not available, the cross-section (c.s.)-scaling model was used. These asymmetries were input to Eq. (3.11.9) to obtain the radiative correction factors. Results from the two models were averaged to provide the final correction $1+\bar{f}_{r c}$, and the difference between the two was combined with uncertainties of resonance models themselves to provide the total uncertainty on $\bar{f}_{r c}$. 
Corrections for the $\gamma \gamma$ box effect to the measured electron asymmetry were estimated to be $\bar{f}_{\gamma \gamma \text { box }}=-0.002$ and -0.003 for DIS \#1 and \#2, respectively. Effect of the $\gamma Z$ box for DIS kinematics was separately treated as part of the electroweak radiative corrections and will be described in Sec. 4.2.2.1. For resonance kinematics, the combined corrections for $\gamma \gamma$ and $\gamma Z$ boxes were estimated to be $\bar{f}_{\gamma \gamma, \gamma Z \text { boxes }}=+0.005$. A relative $100 \%$ uncertainty was used for all box-diagram corrections.

\subsection{Background Analysis}

\subsubsection{Charged Pion Background}

Charged pions are produced from decays of nucleon resonances created by electron scattering off nucleon or nuclear targets. For the pions to have the same momentum as electrons scattered directly off the target, the parent nucleon resonance production must occur at a lower $Q^{2}$ than electron events, thus typically causing a smaller parity-violating asymmetry than that from electrons. This has been confirmed by the asymmetry of the pion triggers measured during the experiment. Furthermore, the high particle identification performance of the DAQ limited the pion contamination in the electron trigger to the level of $f_{\pi / e}<2 \times 10^{-4}$ and $<4 \times 10^{-4}$ for the three DIS kinematics and the five resonance kinematics, respectively [59]. Due to the small contamination, effect of the pions was considered a dilution and no correction to the measured electron asymmetries was made. The total systematic uncertainty on the electron asymmetry due to pion contamination and pion asymmetry is:

$$
\left(\frac{\Delta A_{e}}{A_{e}}\right)_{\pi^{-}}=\sqrt{\left(\Delta f_{\pi / e}\right)^{2}+\left(f_{\pi / e} \frac{\left|A_{\pi}\right|+\Delta A_{\pi}}{A_{e}}\right)^{2}},
$$


where $f_{\pi / e}$ and $\Delta f_{\pi / e}$ are the event fraction of the electron trigger that is from actual pions and its uncertainty, $A_{\pi}$ is the measured pion asymmetry with $\Delta A_{\pi}$ its uncertainty, and $A_{e}$ is the measured electron asymmetry. The term $\left|A_{\pi}\right|+\Delta A_{\pi}$ corresponds to how much the pion asymmetry could differ from zero at the $68.3 \%$ confidence level. As inputs to the background correction, extraction of pion asymmetries is described below.

\section{pion asymmetry measurement}

PID performance of both electron and pion triggers of the DAQ was reported in Ref. [59]. To properly extract pion asymmetries from the trigger, one must account for the effect of electron contamination in the pion triggers, $f_{e / \pi}$. Because $f_{e / \pi}$ was relatively high and the electron asymmetries are larger than those of pions, corrections were applied to the asymmetries extracted from the pion triggers using

$$
A_{\pi}^{\mathrm{phys}}=\frac{A_{\pi, \mathrm{dit}}^{\mathrm{bc}, \mathrm{raw}}-f_{e / \pi} A_{e, \mathrm{dit}}^{\mathrm{bc}, \mathrm{raw}}}{1-f_{e / \pi}}
$$

where $A_{\pi, \text { dit }}^{\text {bc,raw }}$ and $A_{e, \text { dit }}^{\text {bc,raw }}$ are asymmetries extracted from pion and electron triggers, respectively, with beam corrections applied using the dithering method.

\section{electron asymmetry uncertainty due to pion contamination}

Tables 3.10 and 3.11 show respectively for the DIS and the resonance kinematics the beam-corrected and the PID-corrected pion asymmetries, and the total uncertainty on the electron asymmetry due to pion contamination as calculated from Eq. (3.12.1). Values for the pion contamination in electron triggers $f_{\pi / e}$ and the electron contamination in pion triggers $f_{e / \pi}$ and their total uncertainties are from Ref. [59]. 


\begin{tabular}{|c|c|c|c|}
\hline HRS, Kinematics & Left DIS\#1 & Left DIS\#2 & Right DIS\#2 \\
\hline \multicolumn{4}{|c|}{ narrow path } \\
\hline$A_{\pi, \text { dit }}^{\text {bcraw }}(\mathrm{ppm})$ & -57.28 & -26.01 & -21.52 \\
\hline$\Delta A_{\pi, \text { dit }}^{\text {bc,raw }}($ stat. $)(\mathrm{ppm})$ & 7.98 & 14.89 & 4.22 \\
\hline$f_{e / \pi}$ & 0.2653 & 0.0331 & 0.0103 \\
\hline$\Delta f_{e / \pi}($ total $)$ & 0.0603 & 0.0034 & 0.0013 \\
\hline$A_{\pi}^{\text {phys }}(\mathrm{ppm})$ & -48.84 & -21.99 & -20.27 \\
\hline$\Delta A_{\pi}^{\text {phys }}($ total $)(\mathrm{ppm})$ & 13.95 & 21.44 & 6.00 \\
\hline$A_{e, \text { dit }}^{\text {bcraw }}(\mathrm{ppm})$ & -78.45 & -140.30 & -139.84 \\
\hline$A_{e, \text { dit }}^{\text {bc,raw }}($ stat.) $(\mathrm{ppm})$ & 2.68 & 10.43 & 6.58 \\
\hline$f_{\pi / e}\left(\times 10^{-4}\right)$ & 1.07 & 1.97 & 1.30 \\
\hline$\Delta f_{\pi / e}($ total $)\left(\times 10^{-4}\right)$ & 0.24 & 0.18 & 0.10 \\
\hline$\left(\frac{\Delta A_{e}}{A_{e}}\right)_{\pi^{-}, n}$ & $0.89 \times 10^{-4}$ & $0.63 \times 10^{-4}$ & $0.27 \times 10^{-4}$ \\
\hline \multicolumn{4}{|c|}{ wide path } \\
\hline$A_{\pi, \text { dit }}^{\text {bc,raw }}(\mathrm{ppm})$ & -49.60 & -27.04 & -21.41 \\
\hline$\Delta A_{\pi, \text { dit }}^{\text {bc,raw }}($ stat. $)(\mathrm{ppm})$ & 7.67 & 14.89 & 4.22 \\
\hline$f_{e / \pi}$ & 0.2176 & 0.0281 & 0.0091 \\
\hline$\Delta f_{e / \pi}($ total $)$ & 0.0573 & 0.0037 & 0.0013 \\
\hline$A_{\pi}^{\text {phys }}(\mathrm{ppm})$ & -41.29 & -23.71 & -20.30 \\
\hline$\Delta A_{\pi}^{\text {phys }}($ total $)(\mathrm{ppm})$ & 12.84 & 21.38 & 6.00 \\
\hline$A_{e, \mathrm{dit}}(\mathrm{ppm})$ & -78.27 & -140.15 & -140.88 \\
\hline$\Delta A_{e, \text { dit }}$ (stat.) $(\mathrm{ppm})$ & 2.68 & 10.43 & 6.60 \\
\hline$f_{\pi / e}\left(\times 10^{-4}\right)$ & 0.72 & 1.64 & 0.92 \\
\hline$\Delta f_{\pi / e}($ total $)\left(\times 10^{-4}\right)$ & 0.22 & 0.17 & 0.13 \\
\hline$\left(\frac{\Delta A_{e}}{A_{e}}\right)_{\pi^{-}, w}$ & $0.54 \times 10^{-4}$ & $0.55 \times 10^{-4}$ & $0.21 \times 10^{-4}$ \\
\hline
\end{tabular}

Table 3.10: For DIS kinematics: Beam-corrected pion asymmetries $A_{\pi \text {,dit }}^{\mathrm{bc} \text {,raw }}$ with their statistical uncertainties, electron contamination in the pion triggers $f_{e / \pi}$, pion asymmetries results after being corrected for electron contamination $A_{\pi}^{\text {phys }}$, pion contamination in electron triggers $f_{\pi / e}$, and total uncertainties on the electron asymmetry results due to pion background. 


\begin{tabular}{|c|c|c|c|c|c|}
\hline HRS & Left RES I & Left RES II & Right RES III & Left RES IV & Left RES V \\
\hline \multicolumn{6}{|c|}{ narrow path } \\
\hline$A_{\pi, \text { dit }}^{\text {bc,raw }}(\mathrm{ppm})$ & -44.19 & -69.83 & -17.12 & 21.82 & -46.67 \\
\hline$\Delta A_{\pi, \text { dit }}^{\text {bc,raw }}$ (stat.) (ppm) & 40.10 & 26.45 & 8.54 & 47.74 & 64.03 \\
\hline$f_{e / \pi}$ & 0.4114 & 0.3155 & 0.0849 & 0.1852 & 0.1871 \\
\hline$\Delta f_{e / \pi}($ total $)$ & 0.0201 & 0.0163 & 0.0030 & 0.0073 & 0.0077 \\
\hline$A_{\pi}^{\text {phys }}(\mathrm{ppm})$ & -33.68 & -73.15 & -13.50 & 52.15 & -41.46 \\
\hline$\Delta A_{\pi}^{\text {phys }}($ total $)(\mathrm{ppm})$ & 88.58 & 48.82 & 12.69 & 76.19 & 102.42 \\
\hline$A_{e, \mathrm{dit}}$ & -55.11 & -63.75 & -54.38 & -104.04 & -67.87 \\
\hline$\Delta A_{e, \text { dit }}$ (stat.) & 6.77 & 5.91 & 4.47 & 15.26 & 21.25 \\
\hline$f_{\pi / e}\left(\times 10^{-4}\right)$ & 0.79 & 2.40 & 3.82 & 0.26 & 0.45 \\
\hline$\Delta f_{\pi / e}($ total $)\left(\times 10^{-4}\right)$ & 0.11 & 0.20 & 0.23 & 0.03 & 0.03 \\
\hline$\left(\frac{\Delta A_{e}}{A_{e}}\right)_{\pi^{-}, n}$ & $1.75 \times 10^{-4}$ & $4.60 \times 10^{-4}$ & $1.85 \times 10^{-4}$ & $0.32 \times 10^{-4}$ & $0.96 \times 10^{-4}$ \\
\hline \multicolumn{6}{|c|}{ wide path } \\
\hline$A_{\pi, \text { dit }}^{\text {bc,raw }}(\mathrm{ppm})$ & -45.41 & -69.21 & -18.25 & 30.87 & -51.01 \\
\hline$\Delta A_{\pi, \text { dit }}^{\text {bc,raw }}($ stat. $)(\mathrm{ppm})$ & 39.41 & 26.09 & 8.47 & 47.61 & 64.89 \\
\hline$f_{e / \pi}$ & 0.3423 & 0.2409 & 0.0633 & 0.1661 & 0.1598 \\
\hline$\Delta f_{e / \pi}($ total $)$ & 0.0231 & 0.0200 & 0.0060 & 0.0080 & 0.0086 \\
\hline$A_{\pi}^{\text {phys }}(\mathrm{ppm})$ & -39.81 & -71.04 & -15.78 & 58.75 & -47.66 \\
\hline$\Delta A_{\pi}^{\text {phys }}($ total $)(\mathrm{ppm})$ & 74.87 & 43.70 & 12.41 & 74.67 & 101.35 \\
\hline$A_{e, \operatorname{dit}}(\mathrm{ppm})$ & -54.56 & -63.86 & -53.98 & -104.55 & -67.93 \\
\hline$\Delta A_{e, \text { dit }}$ (stat.) $(\mathrm{ppm})$ & 6.77 & 5.91 & 4.46 & 15.26 & 21.46 \\
\hline$f_{\pi / e}\left(\times 10^{-4}\right)$ & 0.54 & 1.50 & 2.14 & 0.22 & 0.32 \\
\hline$\Delta f_{\pi / e}($ total $)\left(\times 10^{-4}\right)$ & 0.15 & 0.25 & 0.48 & 0.03 & 0.04 \\
\hline$\left(\frac{\Delta A_{e}}{A_{e}}\right)_{\pi}$ & $1.13 \times 10^{-4}$ & $2.71 \times 10^{-4}$ & $1.22 \times 10^{-4}$ & $0.28 \times 10^{-4}$ & $0.71 \times 10^{-4}$ \\
\hline
\end{tabular}

Table 3.11: For resonance kinematics: Beam-corrected pion asymmetries $A_{\pi, \text { dit }}^{\text {bc,raw }}$ with their statistical uncertainty, electron contamination in the pion triggers $f_{e / \pi}$, pion asymmetries results after being corrected for electron contamination $A_{\pi}^{\text {phys }}$, pion contamination in electron triggers $f_{\pi / e}$, and total uncertainties on the electron asymmetry results due to the pion background. 


\subsubsection{Pair Production Background}

Pair production background comes from nucleon resonance productions where the resonance decays into neutral pions, then through decay $\pi^{0} \rightarrow e^{+} e^{-}$. The pair production from bremsstrahlung photons is highly forward-peaked and is not significant for the kinematics of this experiment. One therefore expect the pair production background to have an asymmetry determined by the asymmetry of $\pi^{0}$ photo- and electroproductions, which should typically be less in magnitude than electron asymmetry at the same scattered momentum, and comparable to the charged pion asymmetry reported above. The rate of the pair production background was studied during the experiment for the two DIS kinematics by reversing the spectrometer polarity, allowing detection of the positron from the $\pi^{0}$ decay alone. The main focus of such positive polarity runs (or "positron runs") is to precisely determine the fractional contribution from pair production to the main electron trigger, denoted as $f_{e^{+}} / e^{-}$. Due to the relative low rate of positron events, this ratio can be extracted from the HRS DAQ for which the PID performance and rate determination were well understood. Asymmetries of positrons were recorded, however due to the very low rate of positrons the statistical uncertainties were large. In addition, the $\pi^{+}$contamination of the positron triggers was high. This contamination can be determined from PID detector analysis, but the effect on the measured asymmetry from the $\pi^{+}$background cannot be corrected due to lack of knowledge on the asymmetry of $\pi^{+}$itself. Asymmetries extracted from positive polarity runs are shown in Table 3.12 without any correction for the beam fluctuation, beam polarization, or $\pi^{+}$background.

Because the statistical uncertainties of the positron asymmetry results are large, we relied on the fact that $\pi^{0}$ must have similar asymmetries as $\pi^{-}$. We assumed the $\pi^{0}$ asymmetry to be no larger than twice the value of the $\pi^{-}$asymmetry and estimate 


\begin{tabular}{c|c|c}
\hline HRS & Left DIS\#1 & Right DIS\#2 \\
\hline$A_{e^{+}}^{\text {raw }}(\mathrm{ppm})$, narrow & $723.2 \pm 1154.7$ (stat.) & $1216.0 \pm 1304.5($ stat. $)$ \\
$A_{e^{+}}^{\text {raw }}(\mathrm{ppm})$, wide & $742.4 \pm 1151.5($ stat. $)$ & $1199.0 \pm 1304.5($ stat. $)$ \\
\hline
\end{tabular}

Table 3.12: Raw positron asymmetry results. No correction for the beam position, energy, and polarization, or the $\pi^{+}$background was made.

the uncertainty in the electron asymmetry due to pair production background as:

$$
\left(\frac{\Delta A_{e}}{A_{e}}\right)_{\text {pair }}=\sqrt{\left(\Delta f_{e^{+} / e^{-}}\right)^{2}+\left(f_{e^{+} / e^{-}} \frac{\Delta A_{e^{+}}}{A_{e}}\right)^{2}}
$$

where $\Delta A_{e^{+}}$describes how much $A_{e^{+}}$differs from zero and the value $2\left(\left|A_{\pi^{-}}\right|+\Delta A_{\pi^{-}}\right)$ was used. Results for $f_{e^{+}} / e^{-}$and their statistical uncertainties are shown in Table 3.13, and a $10 \%$ uncertainty was used for $\Delta f_{e^{+}} / e^{-}$to account for possible systematic effects in positron identification due to the high $\pi^{+}$background in the rate evaluation. Results for the electron asymmetry uncertainty due to pair production background are also shown in Table 3.13.

\begin{tabular}{c|c|c|c}
\hline HRS & Left DIS\#1 & Left DIS\#2 & Right DIS\#2 \\
\hline$f_{e^{+} / e^{-}}$ & $2.504 \times 10^{-4}$ & $5.154 \times 10^{-3}$ & $4.804 \times 10^{-3}$ \\
$\Delta f_{e^{+}} / e^{-}$(stat.) & $0.007 \times 10^{-4}$ & $0.001 \times 10^{-3}$ & $0.001 \times 10^{-3}$ \\
$\left(\frac{\Delta A_{e}}{A_{e}}\right)_{\text {pair,narrow }}$ & $4.02 \times 10^{-4}$ & $3.23 \times 10^{-3}$ & $1.87 \times 10^{-3}$ \\
$\left(\frac{\Delta A_{e}}{A_{e}}\right)_{\text {pair,wide }}$ & $3.47 \times 10^{-4}$ & $3.36 \times 10^{-3}$ & $1.86 \times 10^{-3}$ \\
\hline
\end{tabular}

Table 3.13: Results for positron contamination $f_{e^{+}} / e^{-}$and its statistical uncertainty, and the total uncertainty on electron asymmetry due to pair production background. Only DIS kinematics were evaluated. The errors shown for $f_{e^{+}} / e^{-}$are statistical only, and a $10 \%$ systematic uncertainty on $f_{e^{+}} / e^{-}$was used in the evaluation of $\frac{\Delta A_{e}}{A_{e}}$.

There was no measurement for the pair production rate for all resonance kinematics. Based on the fact that the $\pi^{-} / e$ rate ratios were smaller in resonance than DIS (see Table 3.1), the effect of the pair production background for the resonance 
kinematics should be no larger than those for DIS. A relative uncertainty of $3 \times 10^{-3}$ was thus used for all resonance asymmetry results.

\subsubsection{Target EndCap Corrections}

Electrons scattered off the target aluminum endcaps cannot be separated from those scattered off the liquid deuterium. Fortunately events from target endcaps belong to similar kinematics as those from deuterium. Based on Eqs. (1.2.4-1.2.11), the value of parity-violating (PV) asymmetry from $e-\mathrm{Al}$ scattering was calculated as

$$
A_{\mathrm{Al}}=\frac{13 A_{p} \sigma_{p}+14 A_{n} \sigma_{n}}{13 \sigma_{p}+14 \sigma_{n}}
$$

where $\sigma_{p(n)}$ is the cross section and $A_{p(n)}$ is the PV asymmetry for scatterings off the proton (neutron). The cross sections $\sigma_{p(n)}$ were calculated using a fit to world resonance and DIS data [74]. The asymmetries $A_{p(n)}$ were calculated using Eq. (1.2.28):

$$
\begin{aligned}
& A_{p}=\left(-\frac{3 G_{F} Q^{2}}{2 \sqrt{2} \pi \alpha}\right) \frac{Y_{1}\left[2 C_{1 u}\left(u^{+}+c^{+}\right)-C_{1 d}\left(d^{+}+s^{+}\right)\right]+Y_{3}\left[2 C_{2 u}\left(u^{-}\right)-C_{2 d}\left(d^{-}\right)\right]}{4\left(u^{+}+c^{+}\right)+\left(d^{+}+s^{+}\right)} \\
& A_{n}=\left(-\frac{3 G_{F} Q^{2}}{2 \sqrt{2} \pi \alpha}\right) \frac{Y_{1}\left[2 C_{1 u}\left(d^{+}+c^{+}\right)-C_{1 d}\left(u^{+}+s^{+}\right)\right]+Y_{3}\left[2 C_{2 u}\left(u^{-}\right)-C_{2 d}\left(d^{-}\right)\right]}{4\left(d^{+}+c^{+}\right)+\left(u^{+}+s^{+}\right)},
\end{aligned}
$$

with $u^{ \pm} \equiv u \pm \bar{u}, d^{ \pm} \equiv d \pm \bar{d}, s^{+} \equiv s+\bar{s}$ and $c^{+} \equiv c+\bar{c}$.

Because aluminum is not an isoscalar nucleus, its asymmetry differs slightly from the deuterium and a correction must be made. Additional uncertainties come from the fact that actual aluminum asymmetries $A_{\mathrm{Al}}$ may differ from the values calculated using Eq. (3.12.4) due to effects such as resonance structure (for resonance kinematics), and nuclear effects similar to the EMC effect $[68,69]$ of the unpolarized, 
parity-conserving structure functions $F_{1,2}$. Based on Ref. [79], the EMC effect for $\mathrm{Al}$ is in the order of $3 \%$ for the two DIS kinematics $(x=0.2-0.3)$, and a conservative relative uncertainty of $10 \%$ was used for $A_{\mathrm{Al}}$ for DIS measurements.

For resonance kinematics, the EMC effect for $\mathrm{Al}$ is in the range $(3-14) \%$, and is larger for higher $x$ values. In addition, the measured electron asymmetry at all five resonance kinematics were found to be in good agreement with the values calculated using PDFs at the 10-15\% level [67], and we expect that the uncertainty in $A_{\mathrm{Al}}$ due to resonance structure cannot exceed this level.. Adding the nuclear and the resonance effects in quadrature, the values of $A_{\mathrm{Al}}$ were estimated to differ no more than $20 \%$ from the values calculated using Eq. (3.12.4). Therefore a $20 \%$ relative uncertainty was used for $A_{\mathrm{Al}}$ for resonance kinematics.

The fractional event rate from the aluminum endcaps, $\alpha_{\mathrm{Al} / \mathrm{D}}$, was calculated as

$$
\alpha_{\mathrm{Al} / \mathrm{D}}=\eta_{\mathrm{Al} / \mathrm{D}} R_{\mathrm{Al} / \mathrm{D}}^{\mathrm{EMC}} \frac{\left(13 \sigma_{p}+14 \sigma_{n}\right) / 27}{\left(\sigma_{p}+\sigma_{n}\right) / 2}=\eta_{\mathrm{Al} / \mathrm{D}} R_{\mathrm{Al} / \mathrm{D}}^{\mathrm{EMC}} \frac{26 \sigma_{p}+28 \sigma_{n}}{27 \sigma_{p}+27 \sigma_{n}}
$$

where $\eta_{\mathrm{Al} / \mathrm{D}}$ is the ratio of the endcap to liquid deuterium thicknesses, and $R_{\mathrm{Al} / \mathrm{D}}^{\mathrm{EMC}}$ is the $\mathrm{Al}$ to deuterium EMC ratio from Ref. $[69,70,71,72,79]$. The target used for this experiment had entrance and exit endcaps measured to be $0.126 \pm 0.011 \pm 0.003 \mathrm{~mm}$ and $0.100 \pm 0.008 \pm 0.003 \mathrm{~mm}$ respectively (see Table 2.1), with the first error bar from the standard deviation of multiple measurements at different positions on the endcap, and the second error from calibration of the instrument. The ratio $\eta_{\mathrm{Al} / \mathrm{D}}$ is $\eta_{\mathrm{Al} / \mathrm{D}}=(0.126+0.100) \mathrm{mm} \times\left(2.7 \mathrm{~g} / \mathrm{cm}^{3}\right) /\left(20 \mathrm{~cm} \times 0.167 \mathrm{~g} / \mathrm{cm}^{3}\right)=1.827 \%$ with an uncertainty of $\Delta \eta_{\mathrm{Al} / \mathrm{D}}=0.115 \%$, or $\Delta \eta_{\mathrm{Al} / \mathrm{D}} / \eta_{\mathrm{Al} / \mathrm{D}}=6.3 \%$. 
The correction to the electron PVDIS asymmetry was applied as

$$
\begin{aligned}
A_{e}^{\mathrm{Al}-\text { corrected }} & =A_{e}\left(1+\bar{f}_{\mathrm{Al}}\right), \\
\text { with } \bar{f}_{\mathrm{Al}} & =-\left(\alpha_{\mathrm{Al} / \mathrm{D}}\right) \frac{A_{\mathrm{Al}}-A_{D}}{A_{D}} .
\end{aligned}
$$

The total uncertainty due to target endcaps is

$$
\left(\frac{\Delta A_{e}}{A_{e}}\right)_{\mathrm{Al}}=\sqrt{\left(\Delta \alpha_{\mathrm{Al} / \mathrm{D}} \frac{A_{A l}-A_{D}}{A_{D}}\right)^{2}+\left[\left(\delta_{A_{\mathrm{Al}}}\right) \alpha_{\mathrm{Al} / \mathrm{D}}\right]^{2}}
$$

where $\Delta \alpha_{\mathrm{Al} / \mathrm{D}}=\left(\Delta \eta_{\mathrm{Al} / \mathrm{D}} / \eta_{\mathrm{Al} / \mathrm{D}}\right) \alpha_{\mathrm{Al} / \mathrm{D}}=0.063 \alpha_{\mathrm{Al} / \mathrm{D}}$ and $\delta_{A_{\mathrm{Al}}}$ is the maximal relative difference in the $\mathrm{Al}$ vs. $\mathrm{D}_{2} \mathrm{PV}$ asymmetries caused by an EMC-like medium modification effect and resonance structures. As stated above, the values $\delta_{A_{\mathrm{A} 1}} \leqslant 10 \%$ for DIS and $\leqslant 20 \%$ for resonances were used. Results for the endcap correction $\bar{f}_{\mathrm{Al}}$ and the uncertainty on the corrected electron asymmetry are given in Table 3.14.

\begin{tabular}{c|c|c|c|c|c|c|c}
\hline Kinematics & DIS\#1 & DIS\#2 & RES I & RES II & RES III & RES IV & RES V \\
\hline$\left(A_{\mathrm{Al}}-A_{D}\right) / A_{D}$ & $0.567 \%$ & $0.727 \%$ & $1.335 \%$ & 0.800 & 0.510 & 0.799 & 0.691 \\
$\alpha_{\mathrm{Al} / \mathrm{D}}$ & $2.02 \%$ & $2.02 \%$ & $2.01 \%$ & $2.02 \%$ & $2.02 \%$ & $2.02 \%$ & $2.02 \%$ \\
$\bar{f}_{\mathrm{Al}}\left(\times 10^{-4}\right)$ & -1.146 & -1.467 & -2.687 & -1.617 & -1.033 & -1.613 & -1.395 \\
$\left(\Delta A_{e} / A_{e}\right)_{\mathrm{Al}}$ & $0.239 \%$ & $0.239 \%$ & $0.422 \%$ & $0.424 \%$ & $0.424 \%$ & $0.423 \%$ & $0.424 \%$ \\
\hline
\end{tabular}

Table 3.14: Relative differences between calculated $\mathrm{Al}$ and $\mathrm{D}_{2}$ asymmetries, $\left(A_{\mathrm{Al}}-\right.$ $\left.A_{D}\right) / A_{D}$, the fractional event rate from $\mathrm{Al}$ endcaps $\alpha_{\mathrm{Al} / \mathrm{D}}$, corrections applied to measured electron asymmetries $\bar{f}_{\mathrm{Al}}$ using Eq. (3.12.9), and the relative uncertainty in the corrected electron asymmetry due to endcap corrections $\left(\Delta A_{e} / A_{e}\right)_{\mathrm{Al}}$.

Events were also taken on a thick, "dummy" target consists of two aluminum foils the thickness approximately 10 times that of the liquid deuterium cell. The thickness was chosen such that the total radiation length of the dummy target matches that of the liquid $\mathrm{D}_{2}$ target. However, due to limited beam time, the asymmetry uncertainty collected from the aluminum dummy target was not precise enough to reduce the 
systematic uncertainty due to target endcaps.

\subsubsection{Beam Transverse Asymmetry Background}

Transverse asymmetry background, also called the beam normal asymmetry background, describes the effect of the electron beam spin polarized in the direction normal to the scattering plane defined by the momentum vectors of the incident and the scattered electrons $\vec{k}_{e}$ and $\vec{k}_{e}^{\prime}[76]$. This beam normal asymmetry is parity-conserving and must be treated as a background of the measurement. Calculations at the pure partonic level show that this asymmetry is between 0.1-0.2 ppm at the kinematics of this experiment, but mechanism beyond the parton level can enhance the asymmetry by 1-2 orders of magnitude [75]. Contribution from the beam normal asymmetry $A_{n}$ to the measured asymmetry can be expressed as

$$
\delta A=\left(A_{n}\right) \vec{S} \cdot \hat{k}_{n} \quad \text { with } \quad \vec{k}_{n} \equiv \hat{k}_{e} \times \hat{k}_{e}^{\prime} \text { and } \hat{k}_{n}=\vec{k}_{n} /\left|\vec{k}_{n}\right|
$$

were $A_{n}$ is the beam-normal asymmetry and $\vec{S}$ is the beam polarization vector. Denoting $\theta_{0}$ the central scattering angle of the spectrometer and $\theta_{t r}$ the average out-ofscattering-plane angle of the spectrometer acceptance, and the Hall coordinates as defined in Fig. 3.23, one has $\hat{k}_{e}=(0,0,1)$ and $\hat{k}_{e}^{\prime}=\left(\sin \theta_{0} \cos \theta_{t r}, \sin \theta_{0} \sin \theta_{t r}, \cos \theta_{0}\right)$, giving $\vec{k}_{n}=\left(-\sin \theta_{0} \sin \theta_{t r}, \sin \theta_{0} \cos \theta_{t r}, 0\right)$ and $\hat{k}_{n}=\left(-\sin \theta_{t r}, \cos \theta_{t r}, 0\right)$, thus

$$
\delta A=A_{n}\left[-S_{H} \sin \theta_{t r}+S_{V} \cos \theta_{t r}\right]
$$

where $S_{V, H, L}$ are respectively the electron polarization components in the vertical (perpendicular to the nominal scattering plane defined by the electron beam and the central ray of the spectrometer), horizontal (within the nominal plane but transverse 
to the beam), and longitudinal directions. During the experiment the beam spin components were controlled to $\left|S_{H} / S_{L}\right| \leqslant 27.4 \%$ and $\left|S_{V} / S_{L}\right| \leqslant 2.5 \%$ and the value of $\theta_{t r}$ was found from data to be less than $0.01 \mathrm{rad}$. Therefore the beam vertical spin dominates this background:

$$
\left(\Delta A_{e}\right)_{A_{n}} \approx A_{n} S_{V} \cos \theta_{t r} \leqslant(2.5 \%) P_{b} A_{n}
$$

where $P_{b}=S_{L}$ is the beam longitudinal polarization described earlier.

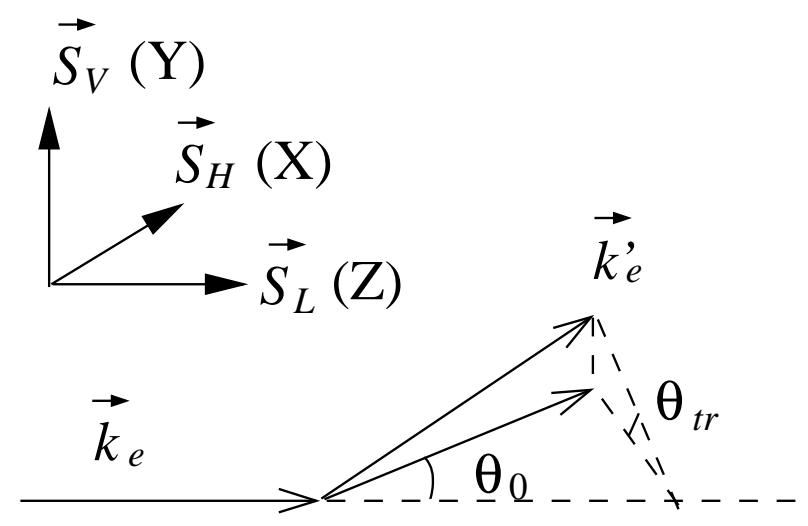

Figure 3.23: Kinematics of the beam normal asymmetry background. The incident and the scattered electrons' momenta are $\vec{k}_{e}$ and $\vec{k}_{e}^{\prime}$, and $\vec{S}_{V, H, L}$ denote respectively the incident electron's spin polarization components in the vertical, horizontal, and longitudinal directions. The central scattering angle of the spectrometer is $\theta_{0}$ and the scattered electron's momentum has an out-of-plane angle denoted by $\theta_{t r}$.

During the experiment, the size of the beam normal asymmetry $A_{n}$ was measured for DIS kinematics during dedicated "transverse runs" where the beam was fully polarized in the vertical direction, $S_{H}=S_{L} \approx 0$ and $S_{V}=P_{b}^{T}$ where $P_{b}^{T}$ is the beam polarization during the transverse asymmetry measurement. Asymmetries measured during these runs are thus $A_{n}^{\text {meas }}=A_{n} P_{b}^{T}$. Since the maximum beam polarization is the same for production and transverse asymmetry running, one has $P_{b}^{T}=S_{0} \equiv$ $\sqrt{S_{L}^{2}+S_{V}^{2}+S_{H}^{2}}=\sqrt{1+(0.274)^{2}+(0.025)^{2}} S_{L}=1.037 S_{L}$ and the total uncertainty 
in the electron asymmetry can be calculated as

$$
\left(\frac{\Delta A_{e}}{A_{e}}\right)_{A_{n}, \mathrm{DIS}}=\frac{\left(\delta A_{n}^{\text {meas }}\right)}{A_{e}^{\text {meas }}} \frac{S_{V}}{S_{0}}=\frac{\delta A_{n}^{\text {meas }}}{A_{e}^{\text {meas }}} \frac{0.025}{1.037}=(2.4 \%) \frac{\delta A_{n}^{\text {meas }}}{A_{e}^{\text {meas }}}
$$

where $A_{e}^{\text {meas }}$ is the measured electron asymmetry and $\delta A_{n}^{\text {meas }}$ describes how much $A_{n}$ could differ from zero. If the measured transverse asymmetry is consistent with zero, the statistical uncertainty of the measurement $\Delta A_{n}^{\text {meas }}$ is taken as $\delta A_{n}^{\text {meas }}$. Otherwise the value of $\left(\left|A_{n}^{\text {meas }}\right|+\Delta A_{n}^{\text {meas }}\right)$ is used as $\delta A_{n}^{\text {meas }}$. Results for the beam transverse asymmetry measurements are shown in Table 3.15 for the two DIS kinematics along with the resulting uncertainty on the electron PVDIS asymmetry due to beam transverse polarizations.

\begin{tabular}{c|c|c}
\hline Kinematics & Left DIS\#1 & Right DIS\#2 \\
$Q^{2}(\mathrm{GeV} / c)^{2}$ & 1.085 & 1.907 \\
\hline$A_{n}^{\text {meas }} \pm \Delta A_{n}^{\text {meas }}(\mathrm{stat}).(\mathrm{ppm}$, narrow $)$ & $-24.15 \pm 15.05$ & $23.49 \pm 44.91$ \\
$A_{e}^{\text {meas }}(\mathrm{ppm}$, narrow $)$ & 78.45 & -139.97 \\
$\left(\frac{\Delta A_{e}}{A_{e}}\right)_{A_{n}, \text { narrow }}$ & $1.18 \%$ & $0.76 \%$ \\
\hline$A_{n}^{\text {meas }} \pm \Delta A_{n}^{\text {meas }}(\mathrm{stat}).(\mathrm{ppm}$, wide $)$ & $-24.66 \pm 15.01$ & $24.60 \pm 44.90$ \\
$A_{e}^{\text {meas }}(\mathrm{ppm}$, wide $)$ & 78.27 & -140.67 \\
$\left(\frac{\Delta A_{e}}{A_{e}}\right)_{A_{n}, \text { wide }}$ & $1.20 \%$ & $0.76 \%$ \\
\hline
\end{tabular}

Table 3.15: Results from the dedicated beam transverse asymmetry measurements and estimation of the total uncertainty on the PVDIS electron asymmetry due to beam transverse polarization. The dithering-corrected values were used for both

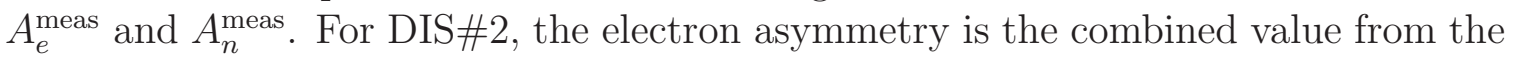
Left and the Right HRS.

No beam transverse measurement was done for the resonance kinematics. However, $A_{n}$ in the DIS region were found to be consistent with previous measurements from electron elastic scattering from the proton and heavier nuclei [76]. Based on this, it was estimated that for all resonance kinematics, $A_{n}$ varies between -38 and $-80 \mathrm{ppm}$ depending on the value of $Q^{2}$, and its amplitude is always smaller than that 
of the parity-violating electron asymmetry due to the longitudinal polarization of the beam. Therefore the uncertainty due to $A_{n}$ was estimated as

$$
\left(\frac{\Delta A_{e}}{A_{e}}\right)_{A_{n}, \mathrm{RES}} \leqslant\left|S_{V} / S_{L}\right|=2.5 \%
$$

\subsubsection{Target Purity, Density Fluctuation and Other False Asymmetries}

The liquid deuterium used contained [77] 1889 ppm HD, < 100 ppm $\mathrm{H}_{2}, 4.4$ ppm $\mathrm{N}_{2}$, $0.7 \mathrm{ppm} \mathrm{O}_{2}, 1.5 \mathrm{ppm} \mathrm{CO},<1 \mathrm{ppm}$ methane and $0.9 \mathrm{ppm} \mathrm{CO}_{2}$. Since most of these are isoscalar nuclei, the only non-negligible effect on the measured asymmetry comes from the proton in HD. The proton asymmetry is given by Eq. (3.12.5) which is well within $\pm(15-30) \%$ of the asymmetry of the deuteron, the proton in HD therefore contributes an uncertainty of less than $\left(\Delta A_{e} / A_{e}\right)_{\mathrm{HD}}<0.06 \%$ to the measured electron asymmetry.

\subsubsection{Rescattering and Poletip Scattering Background}

In this section, two kinds of backgrounds from rescattering inside the HRS spectrometers are considered, both of which are very small.

- Rescattering: Electrons with an energy a few percent different from the accepted electrons which rescatter into the acceptance and make an electron trigger. Their $Q^{2}$ are a few percent different and since the asymmetry $A$ is proportional to $Q^{2}$ it would shift the measured asymmetry. This effect is $\ll 0.2 \%$ of our asymmetry.

- Poletip Scattering: Electrons which may have scattered from the polarized iron (Møller scattering) inside the HRS dipoles (the only location of polarized 
iron). This effect is $\ll 0.1 \mathrm{ppm}$.

The analysis of both these follows Ref. [15], except that in our experiment there is a further suppression by a factor of $>10$ from the fact that our electron trigger requires electrons that pass a high-energy threshold in the leadglass detector, while $>90 \%$ of this background consists of low-energy charged and neutral particles which would not pass the threshold.

For both of these backgrounds, the correction to our asymmetry is, for $f \ll 1$ :

$$
A^{\mathrm{rs}-\text { corrected }}=A^{\text {meas }}-f \Delta A,
$$

where $A^{\text {meas }}$ is the asymmetry we measured, $f$ is the fraction of background, $\Delta A$ is the deviation of the background's asymmetry from the physics asymmetry, and $A^{\text {rs-corrected }}$ is the corrected asymmetry. The correction term is an integral over the energies that contribute to this background:

$$
f \Delta A=\int_{E_{0}^{\prime}-0.2 E_{0}^{\prime}}^{E_{0}^{\prime}+0.2 E_{0}^{\prime}} d E P_{\mathrm{rs}}(E) \times R(E) \times A^{\mathrm{bgr}}
$$

where $E_{0}^{\prime}$ is the nominal setting of the HRS, $P_{\mathrm{rs}}$ is the product of the probability to re-scatter in the HRS and the probability for that particle to cause an electron trigger, $A^{\mathrm{bgr}}$ is the asymmetry of the background, and $R(E)$ is the following ratio

$$
R(E)=\left(\frac{d \sigma}{d \Omega d E}\right)_{\text {outside }} /\left[\Delta E_{\mathrm{HRS}}\left(\frac{d \sigma}{d \Omega d E}\right)_{\text {inside }}\right] .
$$

Here, $\left(\frac{d \sigma}{d \Omega d E}\right)_{\text {inside(outside) }}$ is the differential cross section for the backgrounds from "inside" ("outside") the HRS acceptance and $\Delta E_{\mathrm{HRS}}$ is the HRS energy acceptance. Note that $\Delta E_{\mathrm{HRS}}$ is approximately $\pm 4 \%$ of $E_{0}^{\prime}$. The integral in Eq. (3.12.17) is done for $\pm 20 \%$ of $E_{0}^{\prime}$ because the function $P_{\mathrm{rs}}(E)$ becomes negligible beyond this range. 
Within the $\pm 20 \%$ range of $E_{0}^{\prime}$, the differential cross section does not change significantly, and the integral is computed as a discrete sum with $R(E) d E \approx\left(1 / \Delta E_{\mathrm{HRS}}\right) d E$ at each energy $E$.

The function $P_{\mathrm{rs}}(E)$ has been measured and documented by HAPPEX [15]. The measurement was performed by scanning the magnetic fields in the HRS spectrometer to force the elastically scattered electrons to follow trajectories that simulate electrons with energies $E \neq E_{0}^{\prime}$. The signals in the detectors were measured as a function of the change in the field. The relative signal size as a function of $E$ is assumed to be a crude measure of $P_{\mathrm{rs}}(E)$. The measurements were done both with the counting technique, using the standard HRS DAQ, and with the integrated technique, using the integrating HAPPEX DAQ. For the purpose of the present work, the counting data are relevant. The HAPPEX detector was amplified and sent through a discriminator with a low threshold which was slightly over the noise level. This discriminator signal was used as a trigger to read the detectors in counting mode. From the pulse-height distributions of the HAPPEX detector, the high-energy electrons could be identified. When the HRS was detuned the rate dropped by several orders of magnitude (e.g. $10^{-4}$ for a $12 \%$ mistune), and for the events which passed the low threshold the probability for energy deposition corresponding to the high-energy electrons was less than $10 \%$ [15]. The ADC spectrum from the HAPPEX runs is shown in Fig. 3.24 for runs at the elastic peak and a $14 \%$ detune, and the function $P_{\mathrm{rs}}(E)$ resulting from this analysis is shown in Fig. 3.25.

For the rescattering background, we assume that the asymmetry scales with fourmomentum transfer squared $Q^{2}$.

$$
A^{\mathrm{bgr}} / A^{\text {meas }}=Q_{\mathrm{bgr}}^{2} / Q_{\text {meas }}^{2} .
$$


Calorimeter ADC in Event Mode

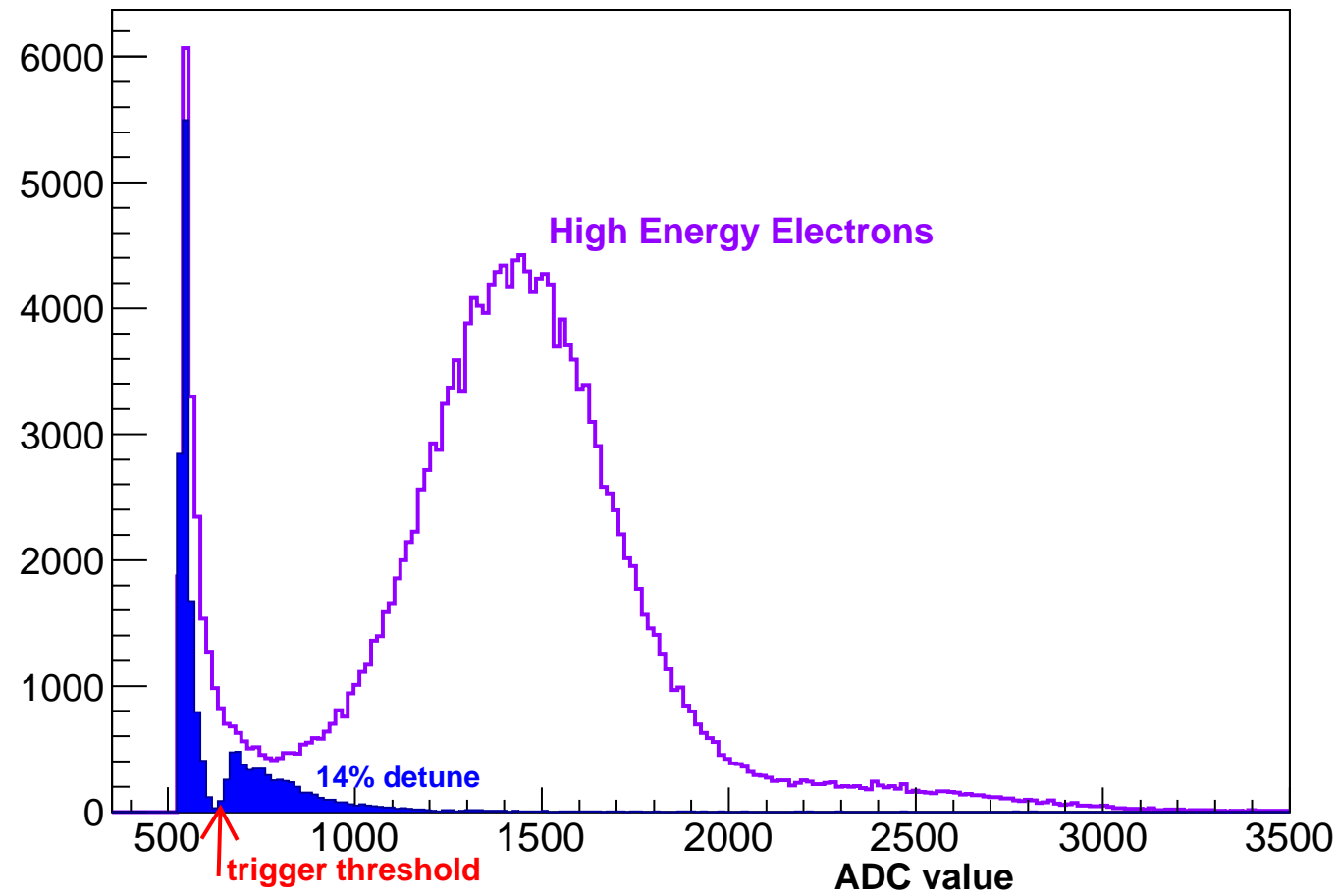

Figure 3.24: ADC spectra of the HAPPEX detector for two runs of the dipole scan [15]. This detector was a kind of calorimeter and recorded the energy deposited. The spectra for the elastic events is labeled "High Energy Electrons". The filled histogram is for the example of a detune by $14 \%$, indicating that the rescattering background is generally formed by lower energy particles. The approximate threshold for the trigger in this study is shown. The threshold for a high-energy electron that corresponds to a PVDIS trigger would be at approximately channel 900 in this spectrum, leading to further suppressions of the background. 


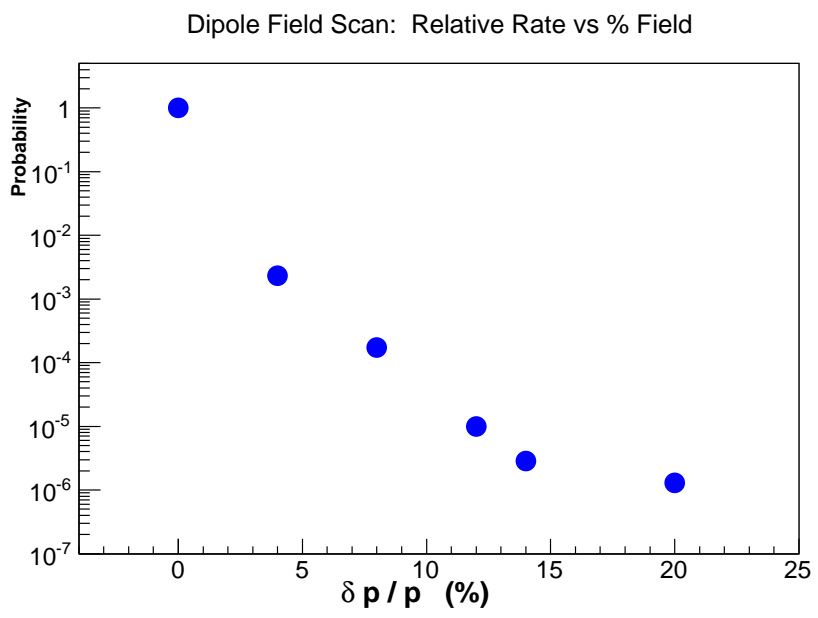

Figure 3.25: The function $P_{\mathrm{rs}}(E)$ determined from HAPPEX data [15]. It is the product of the probability to re-scatter in the HRS and the probability for that particle to cause an electron trigger. Although it wasn't measured symmetrically about zero, we've assumed this symmetry here.

Since $Q^{2}$ varies linearly with the scattered beam energy $E, A^{\text {bgr }}$ varies by $\pm 20 \%$ in Eq. (3.12.17), resulting in $f \Delta A \ll 0.2 \%$. Therefore no correction was made for the rescattering background.

Scattering from the magnetized iron in the HRS dipoles is a potential source of systematic error because of the polarization dependent asymmetry in $(\vec{e}, \vec{e})$ scattering (Møller scattering). In Ref. [15] an upper bound for this effect was found. From the measurements of the function $P_{\mathrm{rs}}$, an electron which rescatters from the last quadrupole $Q_{3}$ corresponds to that function's domain parameter $E$ within $\approx 8 \%$ of $E_{0}$. An electron which rescatters from the polarized iron in the HRS dipole must therefore come from deeper inside the HRS, hence the domain $\left|E-E_{0}^{\prime}\right|>8 \% E_{0}^{\prime}$. In Ref. [15] an upper background of $A_{\mathrm{bgr}}$ was estimated from

$$
A^{\mathrm{bgr}}=P_{e 1} P_{e 2} A_{n}
$$


where $P_{e 1}, P_{e 2}$ are the polarizations of the scattered electron and the electron in the iron $\left(P_{e 1} \approx 0.85\right.$ and $\left.P_{e 2} \approx 0.03\right)$, and $A_{n}$ is the analyzing power $A_{n} \leqslant 0.11$. There is an additional suppression, not assumed in our analysis, for the misalignment of the spins of the two electrons; the scattered electron precess less than $\frac{\pi}{2}$ in the quadrupoles upstream of the dipole, while electron spins in the dipole are oriented along the magnetic field lines. Hence, we find $A^{\text {bgr }}<0.003$. For the poletip scattering candidates, $P_{\mathrm{rs}}$ is found to be very small, $P_{r s}<10^{-5}$. Qualitatively, this is expected for three reasons: (i) the HRS acceptance is defined primarily from the collimators and secondarily by the two quadrupoles that are upstream of the dipole; ray-tracing simulations have shown that, within the approximation of that optics model, the dipole plays no role in defining the apertures; (ii) an electron which scatters in the dipole region would lose energy in the vacuum walls and tend to be swept away; and (iii) the alreadymentioned suppression by a factor $>10$ for the low-energy rescattered particles to make a trigger. From Eq. (3.12.17), and taking into account the assumed $8 \%$ exclusion zone mentioned above, we obtain $f \Delta A \ll 0.1 \mathrm{ppm}$ which is again negligible compared to the statistical uncertainty of the electron asymmetry results. Therefore no correction to the asymmetry was made and the effect of rescattering and pole-tip background was only counted in the evaluation of systematic uncertainties.

\subsection{Resonance Analysis using Group Triggers}

As described in Section 2.6.2, in addition to the global electron trigger, we also had triggers for each individual group. The group triggers were counted by scalers and recorded in the data stream the same way as global trigger. Therefore, we can also extract asymmetry values from these group triggers. According to the grouping scheme implemented in the DAQ system, different groups have different acceptance coverages 
in the dispersive direction of incoming particles, which leads to different values of the kinematic variable $W$ and similar values of $Q^{2}$. Therefore, the group triggers provide a natural binning of $W$ with little variation in $Q^{2}$. For the resonance measurements, group triggers become quite interesting as they might reveal more detailed information about the dependence of parity violation asymmetry on resonance structures.

Asymmetry analysis of group triggers follow the same procedure as the global trigger. The extraction of raw group asymmetries and application of beam corrections were automatically performed along with the global asymmetry analysis during PAN analysis. Most of the corrections described in previous sections for the global trigger remain unchanged for group triggers, except for two of them: the deadtime and the electromagnetic radiative correction. The deadtime depends on the event rate and needs to be recalculated using inputs from each group. This can easily be done using the deadtime simulation (Section 3.7). The radiative correction has a strong dependence on the kinematics coverage and also needs to be reanalyzed, using the same method described in Section 3.11. Table 3.16 summarizes the results of the group asymmetry analysis, including the beam corrected raw asymmetry values, deadtimes and radiative corrections, along with the center kinematic variables $W$ and $Q^{2}$. Other systematic correction factors are the same as in the global asymmetry analysis, and can be found in the corresponding sections of this chapter. 


\begin{tabular}{|c|c|c|c|c|c|c|c|c|c|c|}
\hline \multicolumn{2}{|l|}{ Group } & \multicolumn{2}{|c|}{$\overline{1}$} & 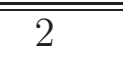 & \multicolumn{2}{|c|}{3} & \multirow[t]{2}{*}{4} & \multirow[t]{2}{*}{5} & \multirow[t]{2}{*}{$\overline{6}$} & \\
\hline \multicolumn{8}{|c|}{ RES I } & & & \\
\hline \multirow{2}{*}{\multicolumn{2}{|c|}{$\begin{array}{c}\left\langle Q^{2}\right\rangle\left[(\mathrm{GeV} / \mathrm{c})^{2}\right] \\
\langle W\rangle(\mathrm{GeV})\end{array}$}} & \multicolumn{2}{|c|}{0.992} & 0.966 & \multicolumn{2}{|c|}{0.948} & 0.940 & 0.931 & \multicolumn{2}{|l|}{0.940} \\
\hline & & \multicolumn{2}{|c|}{1.119} & 1.175 & \multicolumn{2}{|c|}{1.245} & 1.305 & 1.350 & \multicolumn{2}{|l|}{1.364} \\
\hline \multirow{2}{*}{\multicolumn{2}{|c|}{$\begin{array}{c}A_{\text {dit }}^{\text {bc,raw }}(\mathrm{ppm}) \\
\text { (stat.) }\end{array}$}} & \multicolumn{2}{|c|}{-30.84} & -57.65 & \multicolumn{2}{|c|}{-54.01} & -46.12 & -60.24 & \multicolumn{2}{|l|}{-95.49} \\
\hline & & & 31 & 14.34 & 11 & .51 & 11.33 & 14.41 & 23.85 & \\
\hline 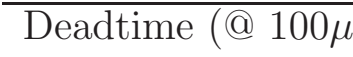 & & 0.7 & $7 \%$ & $0.89 \%$ & 1.0 & $5 \%$ & $1.06 \%$ & $0.88 \%$ & $0.69 \%$ & \\
\hline (syst.) & & 0.0 & $4 \%$ & $0.09 \%$ & 0.0 & $4 \%$ & $0.10 \%$ & $0.08 \%$ & $0.09 \%$ & \\
\hline $1+\overline{f_{r c}}$ & & & 59 & 1.150 & 1.0 & 345 & 1.024 & 1.011 & 1.010 & \\
\hline (syst.) & & & 55 & 0.031 & 0.0 & 14 & 0.005 & 0.004 & 0.004 & \\
\hline & & & & RES I & & & & & & \\
\hline$\left\langle Q^{2}\right\rangle\left[(\mathrm{GeV} / \mathrm{c})^{2}\right]$ & & & 56 & 0.849 & $0 . \varepsilon$ & 334 & 0.820 & 0.808 & 0.819 & \\
\hline$\langle W\rangle(\mathrm{GeV})$ & & & 603 & 1.533 & 1.5 & 583 & 1.629 & 1.662 & 1.672 & \\
\hline$A_{\mathrm{dit}}^{\mathrm{bc}, \mathrm{raw}}(\mathrm{ppm})$ & & & .67 & -55.15 & -77 & .16 & -65.46 & -65.92 & $\begin{array}{l}-61.73 \\
\end{array}$ & \\
\hline (stat.) & & & 24 & 11.18 & 10 & .55 & 10.57 & 12.95 & 20.71 & \\
\hline Deadtime (@ 100 $\mu$ & & & $4 \%$ & $1.52 \%$ & 1.6 & $0 \%$ & $1.58 \%$ & $1.35 \%$ & $1.07 \%$ & \\
\hline (syst.) & & 0.0 & $8 \%$ & $0.17 \%$ & 0.0 & $6 \%$ & $0.14 \%$ & $0.12 \%$ & $0.15 \%$ & \\
\hline $1+f_{r c}$ & & & 32 & 1.017 & 1.0 & 012 & 1.000 & 0.995 & 0.995 & \\
\hline (syst.) & & & 06 & 0.003 & 0.0 & 002 & 0.000 & 0.001 & 0.001 & \\
\hline & & & & RES I & & & & & & \\
\hline$\left\langle Q^{2}\right\rangle\left[(\mathrm{GeV} / \mathrm{c})^{2}\right]$ & & & 31 & 1.533 & 1.4 & 73 & 1.442 & 1.427 & 1.378 & \\
\hline$\langle W\rangle(\mathrm{GeV})$ & & & 01 & 1.922 & 1.9 & 78 & 2.020 & 2.049 & 2.071 & \\
\hline$A_{\mathrm{dit}}^{\mathrm{bc}, \mathrm{raw}}(\mathrm{ppm})$ & & -10 & 3.29 & -91.13 & -82 & .82 & -117.19 & -142.95 & 87.30 & \\
\hline (stat.) & & & 87 & 32.21 & 27 & 24 & 27.00 & 37.52 & 96.85 & \\
\hline Deadtime (@ 100 $\mu$ & & & $7 \%$ & $0.57 \%$ & 0.6 & $1 \%$ & $0.61 \%$ & $0.55 \%$ & $0.49 \%$ & \\
\hline (syst.) & & & $3 \%$ & $0.04 \%$ & 0.0 & $3 \%$ & $0.04 \%$ & $0.04 \%$ & $0.03 \%$ & \\
\hline $1+f_{r c}$ & & & 13 & 1.013 & 1.0 & 20 & 1.027 & 1.031 & 1.032 & \\
\hline (syst.) & & & 03 & 0.003 & 0.0 & 04 & 0.005 & 0.006 & 0.006 & \\
\hline Group & & 1 & 2 & 3 & & 4 & 5 & 6 & 7 & 8 \\
\hline & & & & $\mathrm{RE}$ & III & & & & & \\
\hline$\left\langle Q^{2}\right\rangle\left[(\mathrm{GeV} / \mathrm{c})^{2}\right]$ & & 731 & 0.719 & 0.7 & & 0.744 & 0.761 & 0.777 & 0.796 & 0.799 \\
\hline$\langle W\rangle(\mathrm{GeV})$ & & 928 & 1.923 & 1.9 & & 1.880 & 1.851 & 1.820 & 1.790 & 1.771 \\
\hline$A_{\mathrm{dit}}^{\mathrm{bc}, \mathrm{raw}}(\mathrm{ppm})$ & & 3.62 & -38.74 & -56 & & -56.74 & -56.67 & -57.15 & -52.57 & -35.99 \\
\hline (stat.) & & .82 & 13.05 & 9.9 & & 9.57 & 9.58 & 9.97 & 11.13 & 24.24 \\
\hline Deadtime (@ 100 $\mu \mathrm{A})$ & & $7 \%$ & $1.48 \%$ & 1.6 & & $1.74 \%$ & $1.73 \%$ & $1.70 \%$ & $1.61 \%$ & $1.27 \%$ \\
\hline (syst.) & & $1 \%$ & $0.10 \%$ & 0.1 & & $0.10 \%$ & $0.10 \%$ & $0.10 \%$ & $0.11 \%$ & $0.12 \%$ \\
\hline $1+f_{r c}$ & & 022 & 1.021 & 1.0 & & 1.026 & 1.025 & 1.024 & 1.020 & 1.010 \\
\hline (syst.) & & 004 & 0.004 & 0.0 & & 0.005 & 0.005 & 0.005 & 0.004 & 0.002 \\
\hline
\end{tabular}

Table 3.16: Results from group asymmetry analysis. 


\section{4}

\section{Results and Conclusions}

This chapter summarizes the asymmetry results from previous analysis, and presents the conclusions we can draw from these measurements.

In order to obtain the physics asymmetry, all the systematic corrections must be applied correctly. Assuming each background has asymmetry $A_{i}$ and affected the electron sample with fraction $f_{i}$, the correction can be applied as

$$
A^{\text {phys }}=\frac{\left(\frac{A^{\text {bc,raw }}}{P_{b}}-\sum_{i} A_{i} f_{i}\right)}{1-\sum_{i} f_{i}},
$$

where $A^{\text {bcraw }}$ is the measured asymmetry with helicity-dependent beam corrections applied, and $P_{b}$ is the beam longitudinal polarization presented in Section 3.6. When

all $f_{i}$ are small with $A_{i}$ comparable to or no larger than $A_{\mathrm{bc}, \text { raw }}$, one can define $\bar{f}_{i}=f_{i}\left(1-\frac{A_{i}}{A_{\mathrm{bc}, \text { raw }}} P_{b}\right)$ and approximate

$$
A^{\text {phys }} \approx \frac{A^{\text {bc,raw }}}{P_{b}} \Pi_{i}\left(1+\bar{f}_{i}\right)
$$

i.e., all background corrections were treated as multiplicative. 


\subsection{Resonance Results}

Detailed results of the resonance asymmetries from the global triggers are summarized in Table 4.1, together with the kinematics information as well as a breakdown of the systematic uncertainties. The kinematics shown include the beam energy $E_{b}$, central angle and momentum settings of the spectrometer $\theta_{0}, E_{0}^{\prime}$, and the actual kinematics averaged from the data $\left\langle Q^{2}\right\rangle$ and $\langle x\rangle$. The electron asymmetries obtained from the narrow trigger of the DAQ with beam dithering corrections, $A^{\text {bc,raw }}$, were corrected for the effects from the beam polarization $P_{b}$ and other systematic effects including: the beam depolarization effect $\bar{f}_{\text {depol }}$, the target aluminum endcap $\bar{f}_{\mathrm{Al}}$, the DAQ deadtime $\bar{f}_{\mathrm{dt}}$, the radiative correction $\bar{f}_{\mathrm{rc}}$ that includes effects from energy losses of incoming and scattered electrons as well as the spectrometer acceptance and detector efficiencies, and the box-diagram correction $\bar{f}_{\gamma \gamma, \gamma Z \text { boxes }}$ (for resonances). Other systematic uncertainties that affected the asymmetries include: the charged pion and the pair production background $\bar{f}_{\pi^{-}}$and $\bar{f}_{\text {pair }}$, the beam normal asymmetry $\bar{f}_{A_{n}}$, the uncertainty in the determination of $Q^{2}$, the re-scattering background, and the target impurity. Final results on the physics asymmetries $A^{\text {phys }}$ are shown with their statistical, systematic, and total uncertainties.

In addition to the global trigger, group triggers were also used for extraction of resonance asymmetries (Section 3.13), the results of which are shown in Table 4.2. The group asymmetries are then plotted with different theoretical calculations for comparison, as can be seen in Figure 4.1.

In principle one expects the asymmetry to exhibit distinct structures in the res-

onance region. However, the quark-hadron duality (Section 1.4), if also holds true for weak interactions, implies that the parity violating asymmetries in the resonance region should on average look like natural extensions of the asymmetries from the DIS 


\begin{tabular}{|c|c|c|c|c|c|}
\hline \multicolumn{6}{|c|}{ Kinematics } \\
\hline & RES I & RES II & RES III & RES IV & RES V \\
\hline$E_{b}(\mathrm{GeV})$ & 4.867 & 4.867 & 4.867 & 6.067 & 6.067 \\
\hline$\theta_{0}$ & $12.9^{\circ}$ & $12.9^{\circ}$ & $12.9^{\circ}$ & $15.0^{\circ}$ & $14.0^{\circ}$ \\
\hline$E_{0}^{\prime}(\mathrm{GeV})$ & 4.00 & 3.66 & 3.10 & 3.66 & 3.66 \\
\hline$\left\langle Q^{2}\right\rangle_{\text {data }}\left[(\mathrm{GeV} / c)^{2}\right]$ & 0.950 & 0.831 & 0.757 & 1.472 & 1.278 \\
\hline$\langle x\rangle_{\text {data }}$ & 0.571 & 0.335 & 0.228 & 0.326 & 0.283 \\
\hline$\langle W\rangle_{\text {data }}(\mathrm{GeV})$ & 1.263 & 1.591 & 1.857 & 1.981 & 2.030 \\
\hline$A^{\text {bc,raw }}(\mathrm{ppm})$ & -55.11 & -63.75 & -54.38 & -104.04 & -67.87 \\
\hline (stat.) & \pm 6.77 & \pm 5.91 & \pm 4.47 & \pm 15.26 & \pm 21.25 \\
\hline (syst.) & \pm 0.10 & \pm 0.15 & \pm 0.24 & \pm 0.26 & \pm 0.72 \\
\hline \multicolumn{6}{|c|}{ Corrections with systematic uncertainties } \\
\hline $\bar{P}$ & $90.40 \%$ & $90.40 \%$ & $90.40 \%$ & $89.65 \%$ & $89.65 \%$ \\
\hline$\Delta P_{b}$ & $\pm 1.54 \%$ & $\pm 1.54 \%$ & $\pm 1.54 \%$ & $\pm 1.24 \%$ & $\pm 1.24 \%$ \\
\hline $1+f_{\text {depol }}$ & 1.0005 & 1.0003 & 1.0009 & 1.0006 & 1.0008 \\
\hline (syst.) & $<10^{-4}$ & $<10^{-4}$ & $<10^{-4}$ & $<10^{-4}$ & $<10^{-4}$ \\
\hline $1+f_{\mathrm{Al}}$ & 0.9997 & 0.9998 & 0.9999 & 0.9998 & 0.9999 \\
\hline (syst.) & \pm 0.0042 & \pm 0.0042 & \pm 0.0042 & \pm 0.0042 & \pm 0.0042 \\
\hline $1+f_{\mathrm{dt}}$ & 1.0148 & 1.0247 & 1.0209 & 1.0076 & 1.0095 \\
\hline (syst.) & \pm 0.0006 & \pm 0.0023 & \pm 0.0041 & \pm 0.0004 & 0.0007 \\
\hline $1+f_{\mathrm{rc}}$ & 1.1095 & 1.0205 & 1.0005 & 1.0170 & 1.0134 \\
\hline (syst.) & \pm 0.0352 & \pm 0.0207 & \pm 0.0076 & \pm 0.0112 & 0.0110 \\
\hline $1+f_{\gamma \gamma \text { box }}$ & - & - & - & - & - \\
\hline $1+\bar{f}_{\gamma \gamma, \gamma \text { Zboxes }}$ & 1.005 & 1.005 & 1.005 & 1.005 & 1.005 \\
\hline (syst.) & \pm 0.005 & \pm 0.005 & \pm 0.005 & \pm 0.005 & \pm 0.005 \\
\hline \multicolumn{6}{|c|}{ Other systematic uncertainties in $\Delta A_{\text {phys }} / A_{\text {phys }}$} \\
\hline$\Delta f_{\pi^{-}}$ & $\pm 0.018 \%$ & $\pm 0.046 \%$ & $\pm 0.019 \%$ & $0.003 \%$ & $0.010 \%$ \\
\hline$\Delta \bar{f}_{\text {pair }}$ & $\pm 0.3 \%$ & $\pm 0.3 \%$ & $\pm 0.3 \%$ & $\pm 0.3 \%$ & $\pm 0.3 \%$ \\
\hline$\Delta f_{A_{n}}$ & $\pm 2.5 \%$ & $\pm 2.5 \%$ & $\pm 2.5 \%$ & $\pm 2.5 \%$ & $\pm 2.5 \%$ \\
\hline$\Delta Q^{2}$ & $\pm 0.81 \%$ & $\pm 0.73 \%$ & $\pm 0.87 \%$ & $\pm 3.45 \%$ & $\pm 3.70 \%$ \\
\hline rescatt bg & $\ll 0.2 \%$ & $\ll 0.2 \%$ & $\ll 0.2 \%$ & $\ll 0.2 \%$ & $\ll 0.2 \%$ \\
\hline target impurity & $\pm 0.06 \%$ & $\pm 0.06 \%$ & $\pm 0.06 \%$ & $\pm 0.06 \%$ & $\pm 0.06 \%$ \\
\hline \multicolumn{6}{|c|}{ Asymmetry Results } \\
\hline$A_{\text {phys }}(\mathrm{ppm})$ & -68.62 & -73.75 & -61.49 & -118.97 & -77.50 \\
\hline (stat.) & \pm 8.43 & \pm 6.84 & \pm 5.05 & \pm 17.45 & \pm 24.27 \\
\hline (syst.) & \pm 3.26 & \pm 2.78 & \pm 2.06 & \pm 5.54 & \pm 3.84 \\
\hline (total) & \pm 9.04 & \pm 7.38 & \pm 5.46 & \pm 18.31 & \pm 24.57 \\
\hline
\end{tabular}

Table 4.1: Parity violating asymmetry results for the resonance kinematics. See text for details. 
region. To test if duality works for parity violating asymmetries, we can compare our results to calculations using DIS fits, extrapolated to the $Q^{2}$ and $W$ values of our measurement. This DIS calculation is shown in Figure 4.1 and it is calculated according to Equations $(1.2 .4,1.2 .7,1.2 .8)$ in the following way: the structure functions $F_{1,3}^{\gamma(Z)}$ can be estimated using PDF fits obtained from DIS data, extrapolated to the resonance region, along with the quark- $Z^{0}$ vector and axial couplings $g_{V, A}^{q}$ based on Standard Model values [22]; electroweak radiative corrections were applied to $g_{V, A}^{q}$ directly, and three PDF fits - MSTW [92], CTEQ-Jefferson Lab (CJ) [89] and CT10 [90] - extrapolated to the measured $\left\langle Q^{2}\right\rangle$ and $\langle W\rangle$ values were used along with world data on $R$ [74]. This approach provides DIS estimations $A_{\text {calc }}^{\text {DIS }}$ that can be compared to the measured asymmetries to test quark-hadron duality. One can see from Fig. 4.1 that the measured asymmetries at all kinematics are consistent with the with the DIS estimation, indicating that duality holds for PVES asymmetries in the resonance region. No significant resonance structure is observed in the $W$-dependence of the asymmetries. 


\begin{tabular}{|c|c|c|c|c|c|c|c|c|}
\hline \multicolumn{2}{|c|}{ Group } & 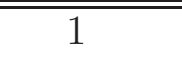 & 2 & 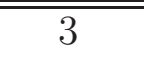 & 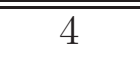 & 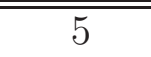 & "6 & \\
\hline \multicolumn{9}{|c|}{$\overline{R E S ~ I ~}$} \\
\hline \multirow{2}{*}{\multicolumn{2}{|c|}{$\begin{array}{c}\left\langle Q^{2}\right\rangle\left[(\mathrm{GeV} / \mathrm{c})^{2}\right] \\
\langle W\rangle(\mathrm{GeV})\end{array}$}} & 0.992 & 0.966 & 0.948 & 0.940 & 0.931 & 0.940 & \\
\hline & & 1.119 & 1.175 & 1.245 & 1.305 & 1.350 & 1.364 & \\
\hline \multirow{2}{*}{\multicolumn{2}{|c|}{$\begin{array}{c}A_{\text {phys }}(\mathrm{ppm}) \\
\text { (stat.) }\end{array}$}} & -46.95 & -74.35 & -63.37 & -53.05 & -68.26 & -107.89 & \\
\hline & & 27.87 & 18.49 & 13.50 & 13.03 & 16.33 & 26.95 & \\
\hline \multicolumn{2}{|c|}{ (syst.) } & 7.42 & 3.36 & 2.26 & 1.77 & 2.26 & 3.58 & \\
\hline \multicolumn{9}{|c|}{ RES II } \\
\hline \multirow{2}{*}{\multicolumn{2}{|c|}{$\begin{array}{c}\left\langle Q^{2}\right\rangle\left[(\mathrm{GeV} / \mathrm{c})^{2}\right] \\
\langle W\rangle(\mathrm{GeV})\end{array}$}} & 0.856 & 0.849 & 0.834 & 0.820 & 0.808 & 0.819 & \\
\hline & & 1.503 & 1.533 & 1.583 & 1.629 & 1.662 & 1.672 & \\
\hline \multirow{3}{*}{\multicolumn{2}{|c|}{$\begin{array}{c}A_{\text {phys }}(\mathrm{ppm}) \\
\text { (stat.) }\end{array}$}} & -70.56 & -63.31 & -88.21 & -73.94 & -73.91 & 69.02 & \\
\hline & & 15.40 & 12.83 & 12.06 & 11.94 & 14.52 & 23.16 & \\
\hline & (syst.) & 2.35 & 2.09 & 2.89 & 2.42 & 2.42 & 2.26 & \\
\hline \multicolumn{9}{|c|}{ RES IV } \\
\hline \multirow{2}{*}{\multicolumn{2}{|c|}{$\begin{array}{c}\left\langle Q^{2}\right\rangle\left[(\mathrm{GeV} / \mathrm{c})^{2}\right] \\
\langle W\rangle(\mathrm{GeV})\end{array}$}} & 1.531 & 1.533 & 1.473 & 1.442 & 1.427 & 1.378 & \\
\hline & & 1.901 & 1.922 & 1.978 & 2.020 & 2.049 & 2.071 & \\
\hline \multicolumn{2}{|c|}{$A_{\text {phys }}(\mathrm{ppm})$} & -118.02 & -104.13 & -95.32 & -135.81 & -166.21 & 101.54 & \\
\hline \multicolumn{2}{|c|}{ (stat.) } & 37.56 & 36.80 & 31.35 & 31.29 & 43.62 & 112.65 & \\
\hline \multicolumn{2}{|c|}{ (syst.) } & 5.43 & 4.79 & 4.39 & 6.28 & 7.70 & 4.71 & \\
\hline Group & $\overline{1}$ & 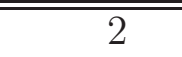 & 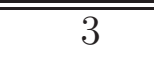 & "4 & 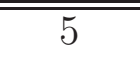 & "6 & $\overline{77}$ & 8 \\
\hline \multicolumn{9}{|c|}{ RES III } \\
\hline$\left.Q^{2}\right\rangle\left[(\mathrm{GeV} / \mathrm{c})^{2}\right]$ & 0.73 & 0.719 & 0.730 & 0.744 & 0.761 & 0.777 & 0.796 & 0.799 \\
\hline$\langle W\rangle(\mathrm{GeV})$ & 1.92 & 1.923 & 1.905 & 1.880 & 1.851 & 1.820 & 1.790 & 1.771 \\
\hline$A_{\text {phys }}(\mathrm{ppm})$ & -67. & $0 \quad-44.66$ & -64.90 & -65.90 & -65.75 & -66.22 & -60.62 & -40.96 \\
\hline (stat.) & 30.8 & 15.05 & 11.53 & 11.12 & 11.12 & 11.55 & 12.83 & 27.59 \\
\hline (syst.) & 2.2 & 1.49 & 2.17 & 2.21 & 2.20 & 2.21 & 2.02 & 1.36 \\
\hline
\end{tabular}

Table 4.2: Group asymmetry results for the resonance measurements. The $W$ and $Q^{2}$ values are averaged over the acceptance of each group. $A_{\text {phys }}$ has all systematic corrections applied. The only corrections that are group-specific are the radiative correction $\bar{f}_{\mathrm{rc}}$ and the deadtime correction $\bar{f}_{\mathrm{dt}}$, which are given in Table 3.16. Other corrections are the same as those for global triggers shown in Table 4.1 


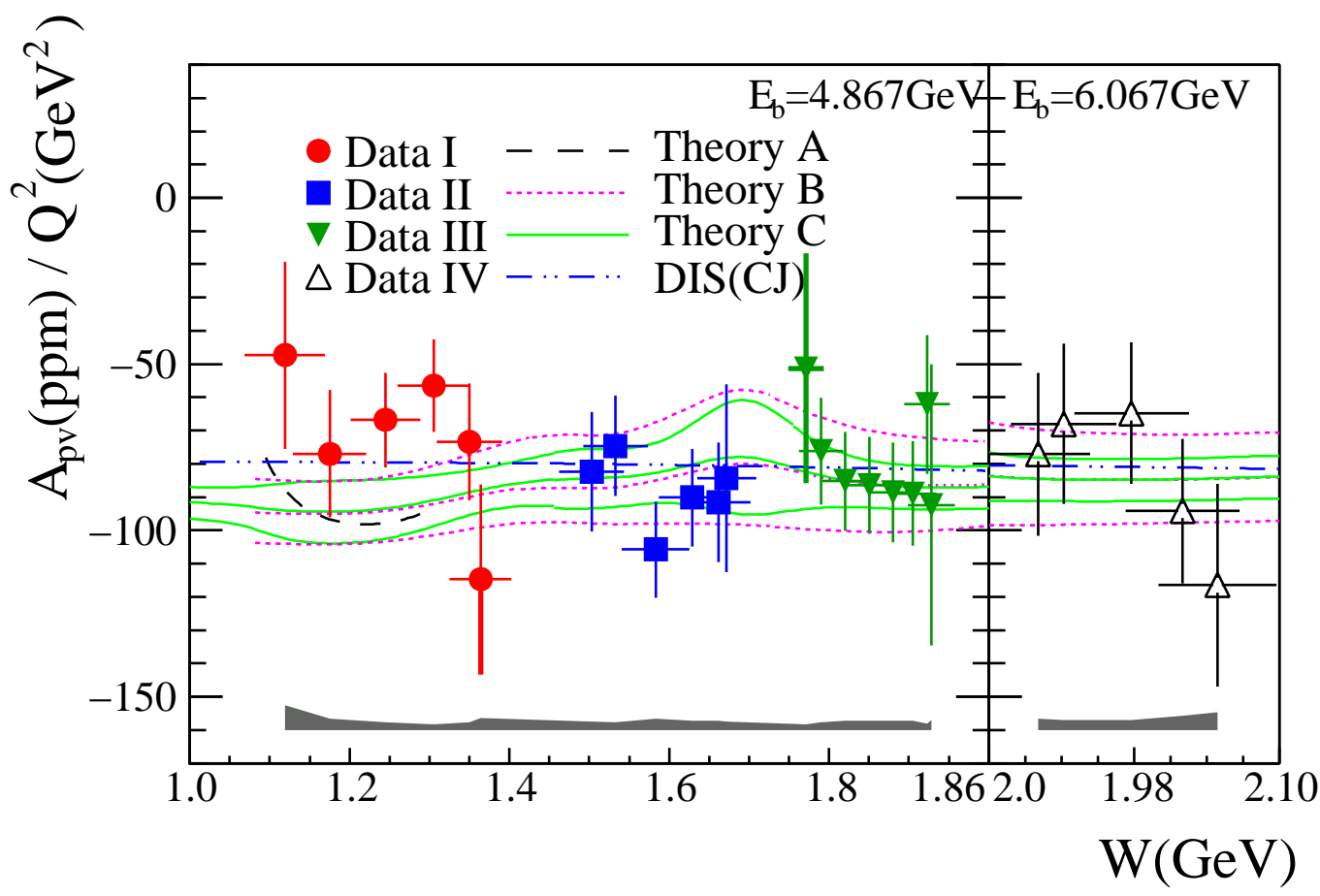

Figure 4.1: $W$-dependence of the parity-violating asymmetries in $\vec{e}-{ }^{2} \mathrm{H}$ scattering extracted from this experiment. The physics asymmetry results $A_{P V}^{\text {phys }}$ for the four kinematics I, II, III and IV (solid circles, solid squares, solid triangles, and open triangles, respectively), in ppm, are scaled by $1 / Q^{2}$ and compared with calculations from Ref. [85] (theory $A$, dashed lines), Ref. [86] (theory $B$, dotted lines), Ref. [87] (theory $C$, solid lines) and the DIS estimation (dash-double-dotted lines) using Equations $(1.2 .4,1.2 .7,1.2 .8)$ with the extrapolated CJ PDF [89]. The vertical error bars for the data are statistical uncertainties, while the horizontal error bars indicate the root-mean-square values of the $W$ coverage of each bin. The experimental systematic uncertainties are shown as the shaded bands at the bottom. For each of the four kinematics, calculations were performed at the fixed $E_{b}$ and $Q^{2}$ values of Table 4.1 and with a variation in $W$ to match the coverage of the data. Theories $B$ and $C$ each have three curves showing the central values and the upper and lower bounds of the calculation. Uncertainties of the DIS calculation were below $1 \mathrm{ppm}$ and are not visible. 


\subsection{DIS Results}

\subsubsection{DIS Asymmetry Results}

Results of the DIS asymmetries are summarized in Table 4.3. After applying all corrections, our final results on the PVDIS asymmetries, in parts per million (ppm or $\left.10^{-6}\right)$, are

$$
\left.\left.A^{P V D I S}=-91.10 \pm 3.11 \text { (stat. }\right) \pm 2.97 \text { (syst. }\right)(\mathrm{ppm}) \text { at } Q^{2}=1.085(\mathrm{GeV} / \mathrm{c})^{2}
$$

$$
\left.A^{P V D I S}=-160.80 \pm 6.39 \text { (stat. }\right) \pm 3.12(\text { syst. })(\mathrm{ppm}) \text { at } Q^{2}=1.901(\mathrm{GeV} / \mathrm{c})^{2}
$$

We can then extract the quark axial coupling combination $2 C_{2 u}-C_{2 d}$ from these asymmetry results.

\subsubsection{Standard Model Calculations And the Extraction of Quark Effective Couplings $2 C_{2 u}-C_{2 d}$}

\subsubsection{Electroweak Radiative Corrections}

Here we discuss the effect of higher order Feynman diagrams including the "running" of all coupling constants involved due to vacuum polarization or loop diagrams, and the effect of the $\gamma Z$ box diagrams of two-boson exchanges. The internal corrections such as vertex corrections and internal bremsstralung, as well as external corrections due to the energy loss of incoming and outgoing electrons, were discussed in section 3.11, and the effect of the $\gamma \gamma$ box diagram was discussed in section 3.11 .3 and will not be repeated here.

The $\alpha_{E M}$ was evaluated at the measured $Q^{2}$ values using $\left.\alpha_{E M}\right|_{Q^{2}=0}=1 / 137.036$ [22]. The 


\begin{tabular}{|c|c|c|c|}
\hline \multicolumn{4}{|c|}{ Kinematics } \\
\hline & DIS\#1 & Left DIS\#2 & Right DIS\#2 \\
\hline$E_{b}(\mathrm{GeV})$ & 6.067 & \multicolumn{2}{|c|}{6.067} \\
\hline$\theta_{0}$ & $12.9^{\circ}$ & \multicolumn{2}{|c|}{$20.0^{\circ}$} \\
\hline$E_{0}^{\prime}(\mathrm{GeV})$ & 3.66 & \multicolumn{2}{|c|}{2.63} \\
\hline$\left\langle Q^{2}\right\rangle_{\text {data }}\left[(\mathrm{GeV} / c)^{2}\right]$ & 1.085 & \multicolumn{2}{|c|}{1.901} \\
\hline$\langle x\rangle_{\text {data }}$ & 0.241 & \multicolumn{2}{|c|}{0.295} \\
\hline$\langle W\rangle_{\text {data }}(\mathrm{GeV})$ & 2.073 & \multicolumn{2}{|c|}{2.330} \\
\hline$A^{\mathrm{bc}, \mathrm{raw}}(\mathrm{ppm})$ & -78.45 & -140.30 & -139.84 \\
\hline (stat.) & \pm 2.68 & \pm 10.43 & \pm 6.58 \\
\hline (syst.) & \pm 0.07 & \pm 0.16 & \pm 0.46 \\
\hline \multicolumn{4}{|c|}{ Corrections with systematic uncertainties } \\
\hline$P_{b}$ & $88.18 \%$ & 89.29 & $88.73 \%$ \\
\hline$\Delta P_{b}$ & $\pm 1.76 \%$ & $1.19 \%$ & $\pm 1.50 \%$ \\
\hline $1+f_{\text {depol }}$ & 1.0010 & \multirow{2}{*}{\multicolumn{2}{|c|}{$\begin{array}{l}1.0021 \\
<10^{-4}\end{array}$}} \\
\hline (syst.) & $<10^{-4}$ & & \\
\hline $1+f_{\mathrm{Al}}$ & 0.9999 & 0.9999 & 0.9999 \\
\hline (syst.) & \pm 0.0024 & \pm 0.0024 & \pm 0.0024 \\
\hline $1+f_{\mathrm{dt}}$ & 1.0147 & 1.0049 & 1.0093 \\
\hline (syst.) & \pm 0.0009 & \pm 0.0004 & \pm 0.0013 \\
\hline $1+f_{\mathrm{rc}}$ & 1.015 & \multirow{2}{*}{\multicolumn{2}{|c|}{$\begin{array}{c}1.019 \\
\pm 0.004\end{array}$}} \\
\hline (syst.) & \pm 0.020 & & \\
\hline $1+f_{\gamma \gamma \text { box }}$ & 0.998 & 0.997 & - \\
\hline $1+\bar{f}_{\gamma \gamma, \gamma \text { Zboxes }}$ & - & - & 1.005 \\
\hline (syst.) & \pm 0.002 & \pm 0.003 & \pm 0.005 \\
\hline \multicolumn{4}{|c|}{ Other systematic uncertainties in $\Delta A_{\text {phys }} / A_{\text {phys }}$} \\
\hline$\Delta f_{\pi^{-}}$ & $\pm 0.009 \%$ & $\pm 0.006 \%$ & $\pm 0.003 \%$ \\
\hline$\Delta \bar{f}_{\text {pair }}$ & $\pm 0.04 \%$ & $\pm 0.3 \%$ & $\pm 0.3 \%$ \\
\hline$\Delta \tilde{f}_{A_{n}}$ & $\pm 2.5 \%$ & $\pm 2.5 \%$ & $\pm 2.5 \%$ \\
\hline$\Delta Q^{2}$ & $\pm 0.85 \%$ & $\pm 0.64 \%$ & $\pm 0.65 \%$ \\
\hline rescatt bg & $\ll 0.2 \%$ & $\ll 0.2 \%$ & $\ll 0.2 \%$ \\
\hline target impurity & $\pm 0.06 \%$ & $\pm 0.06 \%$ & $\pm 0.06 \%$ \\
\hline \multicolumn{4}{|c|}{ Asymmetry Results } \\
\hline$A_{\text {phys }}(\mathrm{ppm})$ & -91.10 & \multirow{4}{*}{\multicolumn{2}{|c|}{$\begin{array}{c}-160.80 \\
\pm 6.39 \\
\pm 3.12 \\
\pm 7.12 \\
\end{array}$}} \\
\hline (stat.) & \pm 3.11 & & \\
\hline (syst.) & \pm 2.97 & & \\
\hline (total) & \pm 4.30 & & \\
\hline
\end{tabular}

Table 4.3: Parity violating asymmetry results for the DIS kinematics. See text of Section 4.1 for details. 
evaluation takes into account purely EM vacuum polarization. The Fermi constant is $G_{F}=1.1663787(6) \times 10^{-5} \mathrm{GeV}^{-2}[22]$. The $C_{1 q, 2 q}$ were evaluated using Table 7 and Eq. (114-115) of Ref. [88] at our measured $Q^{2}$ values in the $\overline{M S}$ scheme using a fixed Higgs mass $M_{H}=125.5 \mathrm{GeV}$. This calculation includes the "charge radius effect" and an estimate of the interference between $\gamma$-exchange and $\gamma Z$ box, but not the effect from the $\gamma \gamma$ box. Effect from the $\gamma \gamma$ box was applied as a correction to the measured asymmetry as described in Sec. 3.11.3.

\subsubsection{Evaluation of Structure Functions and Extraction of $C_{2 q}$}

To express the measured asymmetries in terms of $2 C_{1 u}-C_{1 d}$ and $2 C_{2 u}-C_{2 d}$, we calculated all $F_{1,3}^{\gamma, \gamma Z}$ structure functions in Eqs. (1.2.4,1.2.7-1.2.8) based on parameterizations of parton distribution functions (PDFs). If calculations of the structure functions from PDFs are not available, the quark-parton model was used, as in Eqs. (1.2.11-1.2.15). In this case, leading-order (LO) PDFs were used whenever possible.

The most suitable calculation for our kinematics is from the CTEQ/JLab ("CJ") fit which does provide structure functions directly. However, the CJ fit does not apply to $Q^{2}$ values below $1.7(\mathrm{GeV} / c)^{2}$. To utilize the $Q^{2}=1.085(\mathrm{GeV} / c)^{2}$ asymmetry results, it was necessary to compare the CJ calculation to other PDF fits at $Q^{2}=$ $1.901(\mathrm{GeV} / \mathrm{c})^{2}$ and decide on the best PDF to use for $Q^{2}$ values below $1.7(\mathrm{GeV} / c)^{2}$. Comparison was done among CJ, CT10 [89, 90] and MSTW2008 [91, 92]. It was found that the leading-order MSTW2008 fit gives the closest results to CJ. The variation among all three fits was found to be small, and was used as an estimate of the uncertainty due to structure function calculations. In addition, it is useful to evaluate the value of $a_{1,3}$ assuming that the nucleon is simply made of valence $u$ and $d$ quarks, i.e., using the "no structure" approximation of Eq. (1.2.28). The differences in the 


\begin{tabular}{|c|c|c|}
\hline & $\left\langle\bar{Q}^{2}\right\rangle=1.085,\langle x\rangle=0.241$ & $\left\langle\overline{Q Q}^{2}\right\rangle=1.901,\langle x\rangle=0.295$ \\
\hline \multicolumn{3}{|c|}{ Physical couplings used in the Calculation } \\
\hline$\alpha_{E M}\left(Q^{2}\right)$ & $1 / 134.45$ & $1 / 134.20$ \\
\hline$C_{1 u}^{\mathrm{SM}}$ & -0.1902 & -0.1906 \\
\hline$C_{1 d}^{\mathrm{SM}}$ & 0.3427 & 0.3429 \\
\hline $2 C_{1 u}^{\mathrm{SM}}-C_{1 d}^{\mathrm{SM}}$ & -0.7231 & -0.7241 \\
\hline$C_{2 u}^{\mathrm{SM}}$ & -0.0375 & -0.0380 \\
\hline$C_{2 d}^{\mathrm{SM}}$ & 0.0276 & 0.0280 \\
\hline $2 C_{2 u}^{\mathrm{SM}}-C_{2 d}^{\mathrm{SM}}$ & -0.1025 & -0.1039 \\
\hline \multicolumn{3}{|c|}{$A\left(a_{1}\right), A\left(a_{3}\right)$ terms in ppm } \\
\hline "no structure" & $-83.07,-5.11$ & $-145.49,-14.28$ \\
\hline CTEQ/JLab (CJ) full fit, mid & NA & $-147.37,-12.12$ \\
\hline $\min$ & & $-147.41,-12.99$ \\
\hline $\max$ & & $-147.40,-13.07$ \\
\hline "PDF+QPM" MSTW2008 LO & $-83.61,-4.13$ & $-146.43,-12.48$ \\
\hline "PDF+QPM" CT10 (NLO) & $-84.06,-4.35$ & $-146.64,-12.89$ \\
\hline \multicolumn{3}{|c|}{ coefficients for $2 C_{1 u}-C_{1 d}, 2 C_{2 u}-C_{2 d}$ in ppm } \\
\hline "no structure" & $114.88,49.82$ & $200.92,137.51$ \\
\hline CTEQ/JLab (CJ) full fit, mid & NA & $203.52,116.68$ \\
\hline $\min$ & & $203.58,125.01$ \\
\hline $\max$ & & $203.56,125.78$ \\
\hline "PDF+QPM" MSTW2008 LO & $115.63,40.26$ & $202.22,120.08$ \\
\hline "PDF+QPM" CT10 (NLO) & $116.25,42.41$ & $202.51,124.08$ \\
\hline
\end{tabular}

Table 4.4: Comparison of asymmetry calculation using different structure functions. Values for $\alpha_{E M}\left(Q^{2}\right)$ were calculated using $\alpha_{E M}\left(Q^{2}=0\right)=1 / 137.036$ and $C_{1 q, 2 q}^{\mathrm{SM}}\left(Q^{2}\right)$ were based on Table 7 and Eq. (114-115) of Ref. [88].

calculated asymmetries using PDFs and those using "no structure" approximations provide a scale for the size of PDF-related uncertainties. Values of the $a_{1,3}$ terms of the asymmetries are presented in Sec. 4.4. The uncertainty due to each PDF fit itself is well below 1 ppm.

As one can see from Table 4.4, differences among different fits are below 1 ppm. This is a reasonable estimate of the PDF-related uncertainties since the "no structure" values already do not differ from the results using PDFs by more than $2 \mathrm{ppm}$. Effect of possible differences between $R^{\gamma Z}$ and $R^{\gamma}$ were studied [93]: To account for a shift 
of $1 \mathrm{ppm}$ in the asymmetry, $7.7 \%$ and $4.5 \%$ differences between $R^{\gamma Z}$ and $R^{\gamma}$ are needed, for $Q^{2}=1.085$ and $1.901\left(\mathrm{GeV} / c^{2}\right)$, respectively. Such large differences were considered highly unlikely and the uncertainty in the asymmetry due to the possible difference between $R^{\gamma Z}$ and $R^{\gamma}$ was considered to be negligible compared to statistical uncertainties of the measurement.

Based on the previous discussion, the extraction of the quark weak axial coupling combination $2 C_{2 u}-C_{2 d}$ can be performed using either the CJ fit (for the $Q^{2}=$ $1.901\left(\mathrm{GeV} / \mathrm{c}^{2}\right)$ data point) or the MSTW2008 fit. The extracted results are shown in Table 4.5, together with the statistical and systematic uncertainties. As mentioned before, the uncertainty in asymmetry calculation due to PDF is less than $1 \mathrm{ppm}$, which translates into an uncertainty of less than 0.01 in the extracted $2 C_{2 u}-C_{2 d}$.

\begin{tabular}{c|c|c|c|c}
\hline \hline & $2 C_{2 u}-C_{2 d}$ & stat. & syst. & PDF \\
\hline \multicolumn{5}{c}{$\left\langle Q^{2}\right\rangle=1.901,\langle x\rangle=0.295$} \\
\hline CJ fit & -0.1152 & \pm 0.0548 & \pm 0.0268 & \pm 0.01 \\
MSTW2008, LO & -0.1197 & \pm 0.0513 & \pm 0.0260 & \pm 0.01 \\
SM value & -0.1039 & - & - & - \\
\hline \multicolumn{5}{c}{$\left\langle Q^{2}\right\rangle=1.085,\langle x\rangle=0.241$} \\
\hline MSTW2008, LO & -0.18608 & \pm 0.0773 & \pm 0.0737 & \pm 0.01 \\
SM value & -0.1025 & - & - & - \\
\hline \hline
\end{tabular}

Table 4.5: Results of the quark weak axial coupling combination $2 C_{2 u}-C_{2 d}$ extracted from the asymmetries measured in this experiment. The corresponding Standard Model predicted values are also shown for comparison.

As one can see, the extracted $2 C_{2 u}-C_{2 d}$ results agree well with the Standard Model prediction. The result obtained from the $Q^{2}=1.901\left(\mathrm{GeV} / c^{2}\right)$ data point is also shown in Figure 4.2. Compared with existing measurements, this experiment improves the precision of $2 C_{2 u}-C_{2 d}$ by a factor of five.

It is worth emphasizing that the Standard Model values of $C_{1 q}$ were used during the extraction to obtain the above results. At the time when this thesis is written, a 


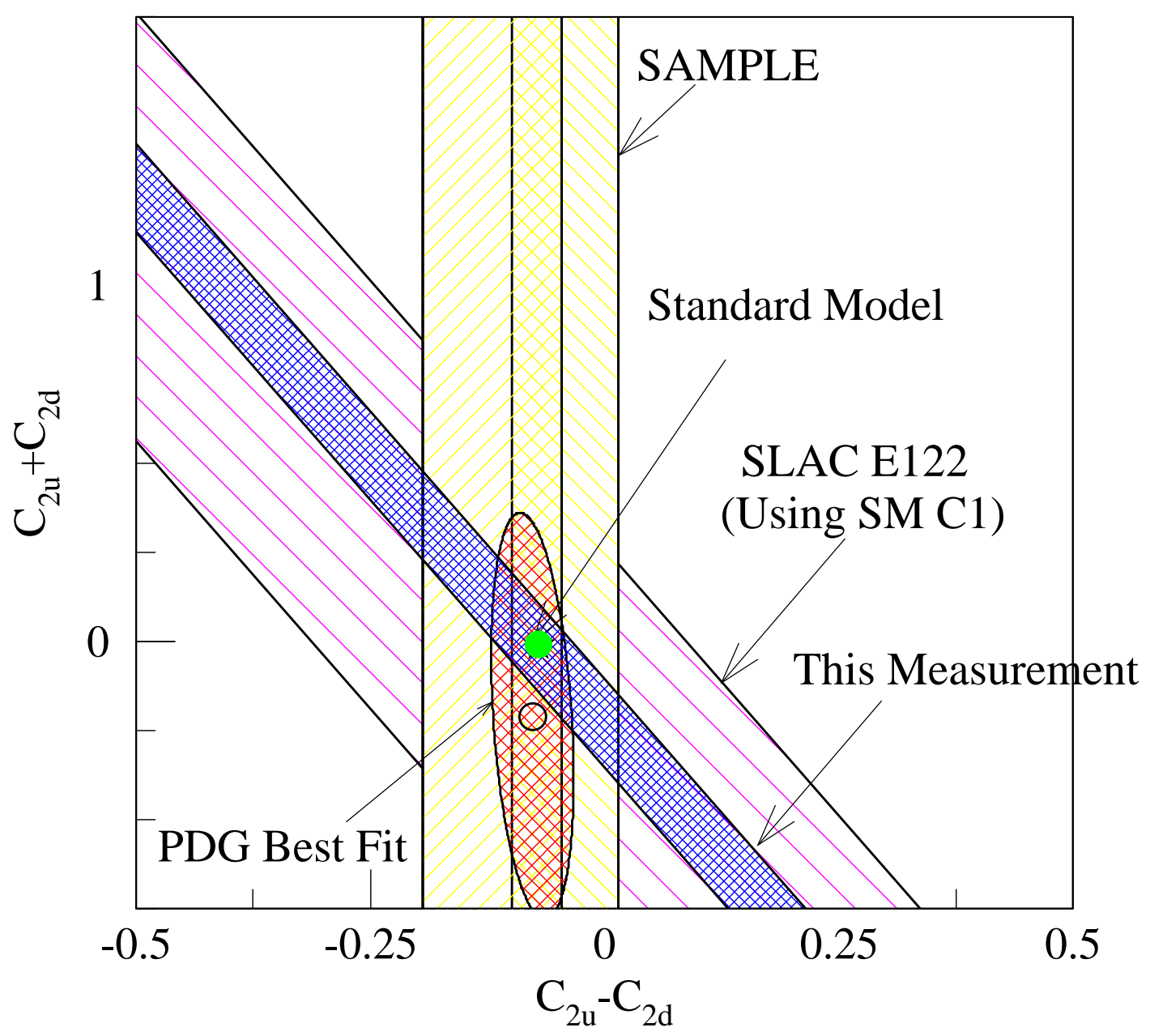

Figure 4.2: Results on quark weak axial coupling combination $2 C_{2 u}-C_{2 d}$ from this measurement, shown as a function of $C_{2 u}+C_{2 d}$ v.s. $C_{2 u}-C_{2 d}$. The result is compared with previous measurements from SLAC (wide shaded band) [4, 5], SAMPLE (the two vertical shaded bands) [82], the Particle Data Group best fit (red ellipse) [12], and the Standard Model prediction (green solid circle). The SLAC results are shown with the same assumption as this experiment that the quark weak vector couplings $C_{1 q}$ agree with the Standard Model. 
more sophisticated fit which simultaneously fits to the $C_{1 q}$ and $C_{2 q}$ using all existing data is being performed [95] and a publication is in preparation.

\subsubsection{The Higher Twist Effect}

The higher twist effects refer to the interaction between quarks inside the nucleon at low $Q^{2}$, where renormalization of the QCD coupling breaks down. At a relative low $Q^{2}$ but not low enough for the effective QCD coupling to diverge, the higher twist effects introduce a $1 / Q^{2}$-dependence to the structure functions in addition to the $\ln Q^{2}$ perturbative QCD evolution. The higher twist effects on $R^{\gamma}$ were estimated in Ref. [94] and the effect on the asymmetry is negligible. Previous data on the higher twist effect of electroweak structure functions $F_{1,3}^{\gamma Z}$ are scarse. The only data that

can be directly applied to $F_{3}^{\gamma Z}$ here are from the neutrino structure function $H_{3}^{\nu}$ [94]. If applying the observed $H_{3}^{\nu}$ higher twist $Q^{2}$ dependence to $F_{3}^{\gamma Z}$ alone, one expects the asymmetry to shift by $+0.70 \mathrm{ppm}$ and $+1.2 \mathrm{ppm}$ for the lower and the higher $Q^{2}$ results, a less than $1 \%$ effect. Moreover, since the non-perturbative interaction between quarks inside the nucleon should not depend on the force-mediating boson (photon or $Z^{0}$ ) exchanged between the quark and the incident electron, one expects a large, if not complete, cancellation between the higher twist terms of $F_{1,3}^{\gamma Z}$ and $F_{1}^{\gamma}$, i.e. the numerator and the denominator of both $a_{1}$ and $a_{3}$ terms. The PVDIS asymmetry should therefore have very small higher twist effect.

The higher twist effect to PVDIS can be investigated through a simultaneous fit to a higher twist coefficient $\beta_{H T}$ and $2 C_{2 u}-C_{2 d}$ using asymmetries measured at the two DIS kinematics during this experiment. The expression

$$
A_{P V}=A_{P V}^{E W}\left(1+\frac{\beta_{H T}}{(1-x)^{3} Q^{2}}\right)
$$




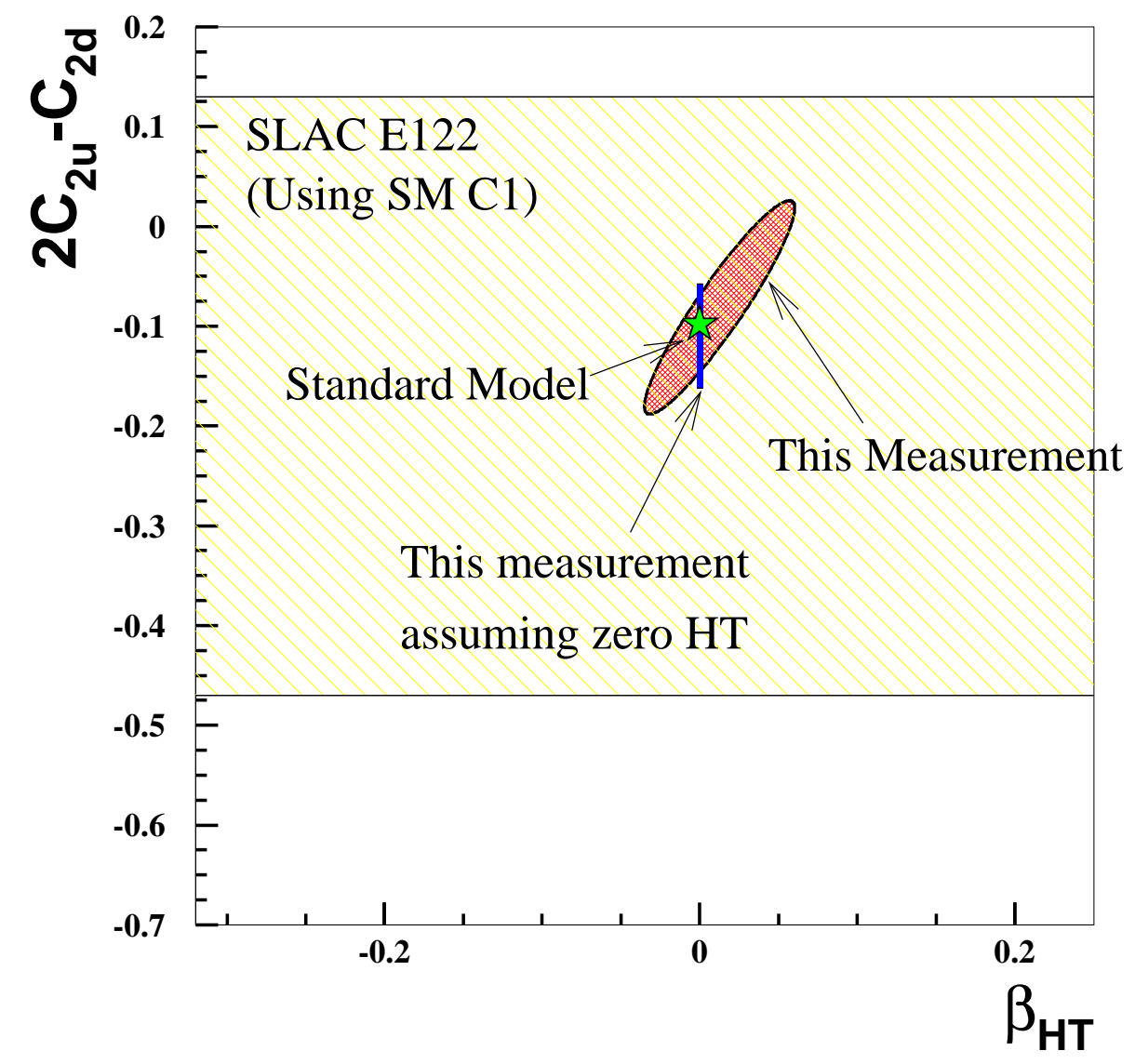

Figure 4.3: Correlation between the higher twist coefficient and $2 C_{2 u}-C_{2 d}$ from asymmetry results of $Q^{2}=1.085$ and $1.901(\mathrm{GeV} / \mathrm{c})^{2}$ (red ellipse), compared with the value of $2 C_{2 u}-C_{2 d}$ assuming $\beta_{H T}=0$ (blue band) and the Standard Model prediction (green star). 
was used where $A_{P V}^{E W}$ is the value calculated based on the Standard Model. The $1 / Q^{2}$ factor is based on the expected $Q^{2}$-dependence of the higher twist term as mentioned above, and the $(1-x)^{3}$ term corresponds to the correlation probability among spectator quarks, although our two DIS measurements have very similar $x$ values which minimizes the sensitivity to this term. The $a_{3}$ term of $A_{P V}^{E W}$ contains $2 C_{2 u}-C_{2 d}$, while the $a_{1}$ term was fixed to the Standard Model values of $2 C_{1 u}-C_{1 d}$. The fit is shown in Figure 4.3 and the result is $\beta_{H T}=0.02598 \pm 0.04723$ and $2 C_{2 u}-$ $C_{2 d}=-0.0602 \pm 0.1090$, with a correlation coefficient 0.91817 . Our result for $\beta_{H T}$ is consistent with zero. This indicates that the extraction of $2 C_{2 u}-C_{2 d}$ is not affected by the higher-twist effect at the present precision.

\subsection{Summary}

In summary, we have measured to a high precision the parity violation asymmetry of $e-{ }^{2} H$ deep inelastic scattering and extracted the quark weak axial coupling combination $2 C_{2 u}-C_{2 d}$. These results are in good agreement with the current Standard Model of elctroweak physics, and show that $2 C_{2 u}-C_{2 d}$ differs from zero by more than two standard deviations, indicating a difference in neutral weak coupling strengths of quarks between their left- and right-handed helicity states.

In addition, we also measured the parity-violating asymmetries in the nucleon resonance region, including the first PV asymmetry data beyond the $\Delta(1232)$ resonance. These results provide important constraints to nucleon resonance models and indicates that quark-hadron duality holds for PVES asymmetries throughout the resonance region. 


\section{Bibliography}

[1] T.Lee, C.-N. Yang, Phys.Rev. 104, 254 (1956). 1

[2] C. Wu, E. Ambler, R.Hayward, D. Hoppes, R.Hudson, Phys.Rev. 105, 1413 (1957). 1

[3] C. Yang, T. Lee, The Nobel Prize in Physics 1957 (1957). 1

[4] C.Y. Prescott et al., Phys. Lett. B 77, (1978) 347. xiii, 1, 15, 153

[5] C.Y. Prescott et al., Phys. Lett. B 84, (1979) 524. xiii, 1, 15, 153

[6] S.L. Glashow, Nucl. Phys. 22, 579 (1961). 1

[7] S. Weinberg, Phys. Rev. Lett. 19, 1264 (1967). 1

[8] A. Salam, Conf. Proc. C 680519, 367 (1968). 1

[9] S.L. Glashow, A. Salam, S. Weinberg, The Nobel Prize in Physics 1979 (1979). 1

[10] CERN experiments observed particle consistent with the long-sought Higgs boson (2012). 2

[11] F. Englert, P.W. Higgs, The Nobel Prize in Physics 2013 (2013). 2

[12] K. Nakamura et al. J. Phys. G 37, 075021 (2010). xiii, 3, 153

[13] J.D. Bjorken, E.A. Paschos, Phys. Rev. 185, 1975 (1969). 8

[14] R. D. McKeown, Phys. Lett. B 219 (1989) 140; D.T. Spayde, et al. Phys. Lett. B583 (2004) 79; T. Ito, et al. Phys. Rev. Lett. 92 (2004) 102003. 10, 15

[15] K. A. Aniol et al. [HAPPEX Collaboration], Phys. Rev. C 69, 065501 (2004). xiii, 10, 15, 117, 135, 136, 137, 138

[16] K.A. Aniol et al., Phys. Lett. B 509 (2001) 211; K. A. Aniol et al. [HAPPEX Collaboration], Phys. Rev. Lett. 96, 022003 (2006); K. A. Aniol et al. [HAPPEX Collaboration], Phys. Lett. B 635, 275 (2006); A. Acha et al. [HAPPEX Collaboration], Phys. Rev. Lett. 98, 032301 (2007). 10, 15, 33 
[17] Z. Ahmed et al. [HAPPEX Collaboration], Phys. Rev. Lett. 108, 102001 (2012). $10,15,69,105$

[18] F.E. Maas et al. [A4 Collaboration], Phys. Rev. Lett. 93, 022002 (2004). F.E. Maas et al. [A4 Collaboration], Phys. Rev. Lett. 94, 152001 (2005). S. Baunack et al. [A4 Collaboration], Phys. Rev. Lett. 102, 151803 (2009). 10, 15

[19] D.H. Beck, Phys. Rev. D 39 (1989) 3248; D.S. Armstrong et al., Phys. Rev. Lett. 95 (2005) 092001; D. Androic et al., Phys. Rev. Lett. 104 (2010) 012001. 10, 15

[20] S. Abrahamyan, et al. Phys. Rev. Lett. 108, 112502 (2012); C.J. Horowitz, et al. Phys. Rev. C 85, 032501(R) (2012). 10, 15, 69

[21] R. N. Cahn and F. J. Gilman, Phys. Rev. D 17, 1313 (1978). 11, 17

[22] J. Beringer et al. (Particle Data Group), Phys. Rev. D86, 010001 (2012). 14, $145,148,150$

[23] Wood, C.S., Bennett, S.C., Cho, D., Masterson, B.P., Roberts, J.L., Tanner, C.E. andWieman, C.E., Measurement of parity nonconservation and an anapole moment in cesium, Science 275, 1759 (1997); Bennett, S.C. and Wieman, C.E., Measurement of the ${ }^{6} S \rightarrow{ }^{7} S$ transition polarizability in atomic cesium and an improved test of the Standard Model, Phys. Rev. Lett. 82, 2484 (1999) [Erratumibid. 83, 889 (1999)]; Ginges, J.S.M. and Flambaum, V.V., Violations of fundamental symmetries in atoms and tests of unification theories of elementary particles, Phys. Rept. 397, 63 (2004); V.A. Dzuba, J.C. Berengut, V.V. Flambaum and B. Roberts, Phys. Rev. Lett. 109, 203003 (2012). 15

[24] D. Androic, et al. [QWeak Collaboration], First Determination of the Weak Charge of the Proton, Phys. Rev. Lett. 111, 141803 (2013). viii, 15, 16, 17

[25] R. Michaels, P.E. Reimer, X. Zheng et al., Jefferson Lab E08-011, $\vec{e}-{ }^{2} \mathrm{H}$ Parity Violating Deep Inelastic Scattering (PVDIS) at CEBAF $6 \mathrm{GeV}$. 16, 19

[26] E. D. Bloom and F. J. Gilman, Phys. Rev. Lett. 25, 1140 (1970). 17

[27] I. Niculescu et al., Phys. Rev. Lett. 85, 1186 (2000). 17

[28] Y. Liang et al., nucl-ex/0410027. 17

[29] A. Psaker, W. Melnitchouk, M. E. Christy, and C. Keppel, Phys. Rev. C 78, 025206 (2008). 17

[30] S. P. Malace, Y. Kahn, W. Melnitchouk and C. E. Keppel, Phys. Rev. Lett. 104, 102001 (2010). 17

[31] S. P. Malace et al., Phys. Rev. C 80, 035207 (2009). 17 
[32] A. Airapetian et al. (HERMES Collaboration), Phys. Rev. Lett. 90, 092002 (2003). 17

[33] M. J. Musolf et al., Phys. Rep. 239, 1 (1994); J. E. Amaro, M. B. Barbaro, J. A. Caballero, T. W. Donnelly and A. Molinari, Phys. Rept. 368, 317 (2002); M. B. Barbaro, J. A. Caballero, T. W. Donnelly and C. Maieron, Phys. Rev. C 69, 035502 (2004); A. De Pace, M. Nardi, W. M. Alberico, T. W. Donnelly and A. Molinari, Nucl. Phys. A741, 249 (2004); S. L. Adler, Ann. Phys. (N.Y.) 50, 189 (1968); D. R. T. Jones and S. T. Petcov, Phys. Lett. 91B, 137 (1980); L. M. Nath, K. Schilcher and M. Kretzschmar, Phys. Rev. D 25, 2300 (1982). 17

[34] D. Androic et al., arXiv:1212.1637, compiled for Phys. Lett. B. 17

[35] P. E. Bosted et al., Phys. Rev. C 75, 035203 (2007). 18

[36] P. Solvignon et al., Phys. Rev. Lett. 101, 182502 (2008). 18

[37] S. P. Malace, W. Melnitchouk, and A. Psaker, Phys. Rev. C 83, 035203 (2011). 18

[38] T. Navasardyan et al., Phys. Rev. Lett. 98, 022001 (2007). 18

[39] C. E. Carlson and N. C. Mukhopadhyay, Phys. Rev. D 47, 1737 (1993). 18

[40] C. K. Sinclair, et al. Phys. Rev. ST Accel. Beams 10, 023501 (2007); J. Hansknecht, et al. Phys. Rev. ST Accel. Beams 13, 010101 (2010). 20

[41] J. Grames, et al., Proceedings of the 2011 Particle Accelerator Conference, New York, New York (2011). 21

[42] O. Ravel, Ph.D. Thesis, Universite Blaise Pascal, Clermont-Ferrand, France (1997). 25

[43] J. Alcorn et al., Nucl. Instrum. Meth. A 522, 294 (2004). 26, 33, 40, 43, 106, 109

[44] K. D. Paschke, Eur. Phys. J. A 32, 549 (2007). 28

[45] W. Barry, Nucl. Instrum. Meth. A. 301, 407 (1991); T. Powers, L. Doolittle, R. Ursic, and J. Wagner, Proc. 7th Workshop on Beam Instrumentation, AIP Conf.Proc. 390, Ed. A.. Lumpkin and C.E. Eyberger (1997); JLAB-TN-96-021. 29

[46] R. Silwal, Ph.D. Thesis, University of Virginia (2012). 30, 54

[47] J. S. Price, B. M. Poelker, C. K. Sinclair, P. M. Rutt, D. J. Mack and J. M. Grames, In Protvino 1998, High energy spin physics, 554-556. 32 
[48] J. S. Price, B. M. Poelker, C. K. Sinclair, K. A. Assamagan, L. S. Cardman, J. Grames, J. Hansknecht and D. J. Mack et al., In Urbana 1997, Polarized gas targets and polarized beams, 446-450. 32

[49] M. Steigerwald, http://www.jlab.org/accel/inj_group/mott/mott.pdf. 32

[50] C.K. Sinclair, "Electron Beam Polarimetry", Tech Note JLAB-ACC-98-04; T.J. Gay and F.B. Dunning, Rev. Sci. Instrum.63 (1992) 1635; T.J. Gay et al., Rev. Sci. Instrum. 63 (1992) 114. S. Mayer et al., Rev. Sci. Instrum. 64 (1993) 952. 33

[51] M. Baylac, E. Burtin, C. Cavata, S. Escoffier, B. Frois, D. Lhuillier, F. Marie and J. Martino et al., Phys. Lett. B 539, 8 (2002). 36

[52] D. Neyret, T. Pussieux, T. Auger, M. Baylac, E. Burtin, C. Cavata, R. Chipaux and S. Escoffier et al., Nucl. Instrum. Meth. A 443, 231 (2000). 36

[53] M. Friend, D. Parno, F. Benmokhtar, A. Camsonne, M. Dalton, G. B. Franklin, V. Mamyan and R. Michaels et al., Nucl. Instrum. Meth. A 676, 96 (2012). 36, $38,77,78$

[54] M. Lynn Friend, Ph.D. Thesis, Carnegie Mellon University (2012). 36, 38, 77, 78

[55] J. P. Jorda, E. Burtin, C. Cavata, J. Jardillier, B. Frois, D. Neyret, J. Martino and S. Platchkov et al., Nucl. Instrum. Meth. A 412, 1 (1998). 37

[56] W. Bertozzi et al. Nucl. Instr. and Meth. 191, 957 (1977). 47

[57] K.G. Fissum et al. Nucl. Instr. and Meth. A, 474, 108 (2001). 47

[58] W.A. Watson et al., CODA: A Scalable, Distributed Data Acquisition System, in Proc. of the Real Time 1993 Conference, p. 296; G. Heyes et al., The CEBAF Online Data Acquisition System, in Proc. of the CHEP Conference, 1994, p. 122; D.J. Abbott et al., CODA Performance in the Real World, 11th IEEE NPSS Real Time 199 Conference, JLab-TN-99-12 (1999). 52

[59] R. Subedi, D. Wang, K. Pan, X. Deng, R. Michaels, P. E. Reimer, A. Shahinyan, B. Wojtsekhowski and X. Zheng, "A Scaler-Based Data Acquisition System for Measuring Parity Violation Asymmetry in Deep Inelastic Scattering," Nucl. Instrum. Meth. A. 724, 90 (2013). 58, 101, 122, 123

[60] ROOT/C++ Analyzer for Hall A, https://hallaweb.jlab.org/podd/index.html 61

[61] ROOT, http://root.cern.ch/drupal/ 61 
[62] PAN: The Parity Analyzer, http://www.phy.syr.edu/ rsholmes/happex/pan/html/index.html 61

[63] Bryan Moffit, Ph.D. Thesis, College of William and Mary (2007). 72

[64] PVDIS electronic logbook, http://ace.phys.virginia.edu/PVDIS/110. 83

[65] Kai Pan, M.S. Thesis, MIT (2013). 101

[66] N. Liyanage, Spectrometer constant determination for the Hall A HRS pair, JLab-TN-01-049, 2001. 101, 102

[67] D. Wang et al. [Jefferson Lab Hall A Collaboration], Phys. Rev. Lett. 111, 082501 (2013). 118, 129

[68] J. J. Aubert et al. [European Muon Collaboration], Phys. Lett. B 123, 275 (1983). 128

[69] P. R. Norton, Rept. Prog. Phys. 66, 1253 (2003). 128, 129

[70] J. Gomez, R. G. Arnold, P. E. Bosted, C. C. Chang, A. T. Katramatou, G. G. Petratos, A. A. Rahbar and S. E. Rock et al., Phys. Rev. D 49, 4348 (1994). 129

[71] S. Stein, W. B. Atwood, E. D. Bloom, R. L. Cottrell, H. C. DeStaebler, C. L. Jordan, H. Piel and C. Y. Prescott et al., Phys. Rev. D 12, 1884 (1975). 129

[72] S. Rock and P. E. Bosted, Phys. Lett. B 518, 34 (2001). 129

[73] M. Arneodo et al. [New Muon. Collaboration], Phys. Lett. B 364, 107 (1995). 118

[74] P. E. Bosted and M. E. Christy, Phys. Rev. C 77, 065206 (2008). 118, 128, 145

[75] A. Afanasev, priv. communication. 131

[76] S. Abrahamyan et al. [HAPPEX and PREX Collaborations], Phys. Rev. Lett. 109, 192501 (2012). 131, 133

[77] M. Seely, Gas Chromatograph Analysis for Deuterium Sample, July 26, 2002. 134

[78] H. Olsen and L. C. Maximon, Phys. Rev. 114, 887 (1959). 113

[79] I. Sick and D. Day, Phys. Lett. B 274, 16 (1992). 129

[80] L. W. Mo and Y. -S. Tsai, Rev. Mod. Phys. 41, 205 (1969). 116

[81] R. Michaels, Formulas for Hall A Monte Carlo (2008), URL: http://hallaweb.jlab.org/parity/prex/hamc/hamc_formulas.pdf. 116 
[82] E. J. Beise, M. L. Pitt and D. T. Spayde, Prog. Part. Nucl. Phys. 54, 289 (2005). xiii, 117,153

[83] D. Abbott et al. [JLAB t20 Collaboration], Eur. Phys. J. A 7, 421 (2000). 117

[84] S. J. Pollock, Phys. Rev. D 42, 3010 (1990), [Erratum-ibid. D 43, 2447 (1991)]. 117

[85] K. Matsui, T. Sato and T. -S. H. Lee, Phys. Rev. C 72, 025204 (2005). xiii, 118, 121,147

[86] M. Gorchtein, C. J. Horowitz and M. J. Ramsey-Musolf, Phys. Rev. C 84, 015502 (2011). xiii, 118, 121, 147

[87] N.L. Hall, P.G. Blunden, W. Melnitchouk, A.W. Thomas and R.D. Young, Phys. Rev. D 88, 013011 (2013). xiii, 147

[88] J. Erler and S. Su, arXiv:1303.5522 [hep-ph]. xvii, 150, 151

[89] J. F. Owens, A. Accardi, and W. Melnitchouk, Phys. Rev. D 87, 094012 (2013). xiii, $145,147,150$

[90] H. -L. Lai, M. Guzzi, J. Huston, Z. Li, P. M. Nadolsky, J. Pumplin and C. P. Yuan, Phys. Rev. D 82, 074024 (2010). 145, 150

[91] A. D. Martin, W. J. Stirling, R. S. Thorne and G. Watt, Phys. Lett. B 652, 292 (2007). 150

[92] A. D. Martin, W. J. Stirling, R. S. Thorne and G. Watt, Eur. Phys. J. C 63, 189 (2009). 118, 145, 150

[93] T. Hobbs and W. Melnitchouk, Phys. Rev. D 77, 114023 (2008). 151

[94] S. Alekhin, S. A. Kulagin and R. Petti, AIP Conf. Proc. 967, 215 (2007). 154

[95] The work is being carried out by Jens Erler. 154 